no.

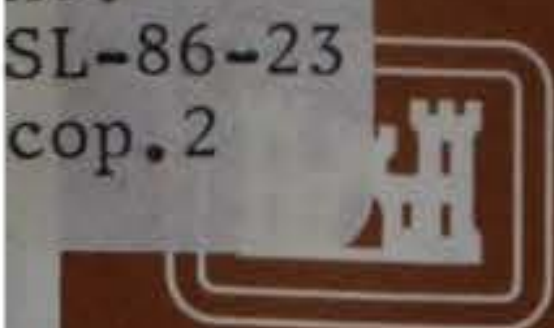

US Army Corps of Engineers
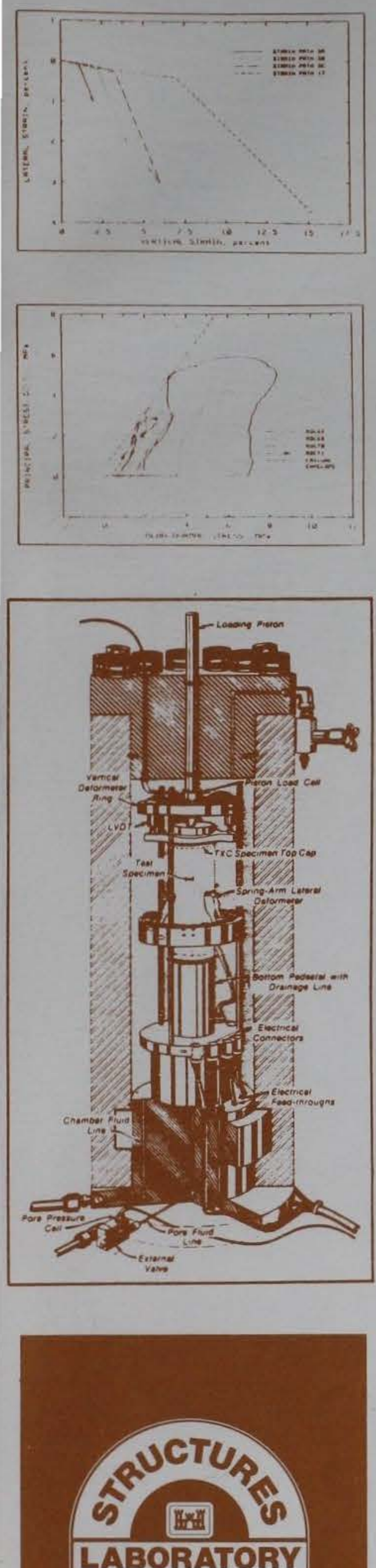

TECHNICAL REPORT SL-86-23

\section{AXISYMMETRIC STRAIN-PATH AND STRESS-PATH TESTS ON CARES-DRY CLAYEY SAND}

by

Stephen A. Akers

Structures Laboratory

DEPARTMENT OF THE ARMY

Waterways Experiment Station, Corps of Engineers PO Box 631, Vicksburg, Mississippi 39180-0631

\section{US-CE-Cproperty of the United States Government}

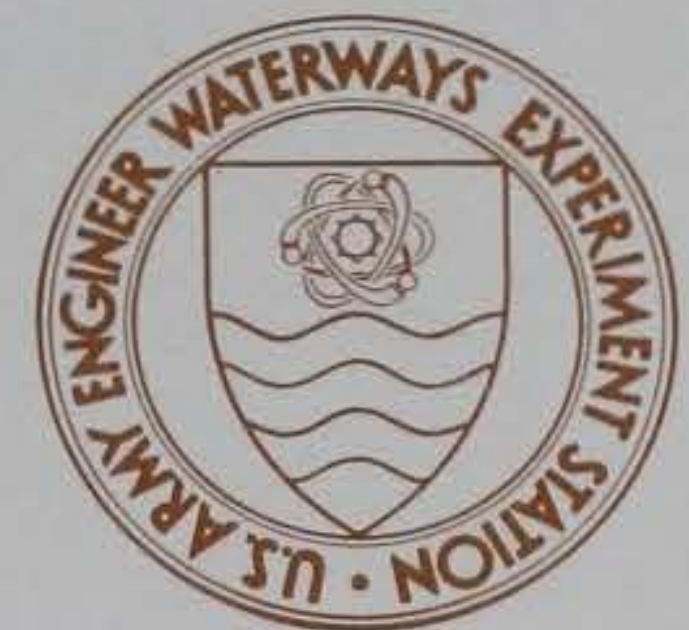

September 1986 Final Report Approved For Public Release; Distribution Unlimited Library Branch Technical information Center U.S. Army Engineer Waterways Experiment Station Vicksburg, Mississippi

Prepared for Defense Nuclear Agency Washington, DC 20305 Under DNA Task Code Y99QMXSB, Work Unit 00003 
Unclassified

SECURITY CLASSIFICATION OF THIS PAGE

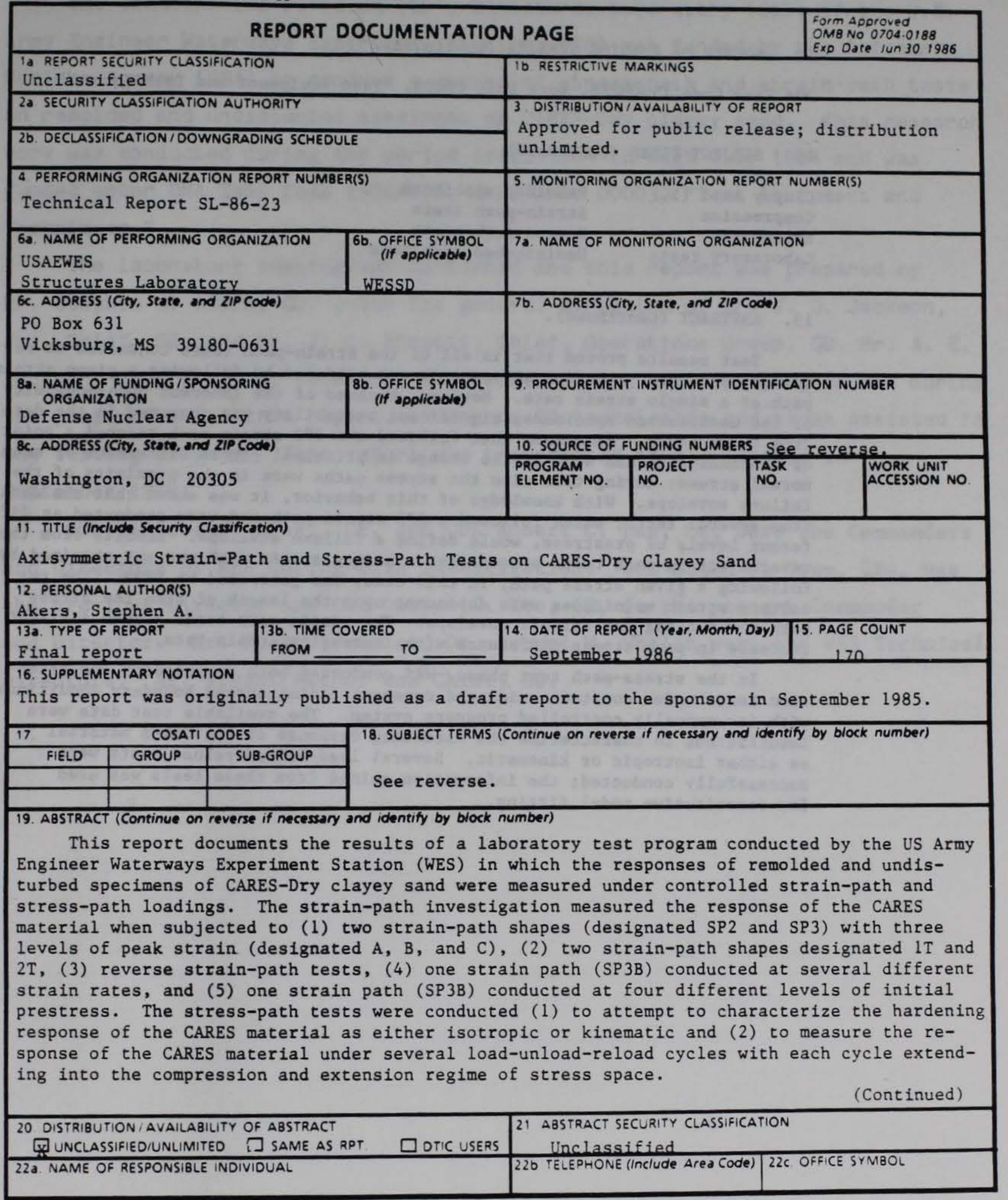

DD FORM 1473, 84 MAR

83 APR edition may be used until exhausted

All other editions are obsolete

SECURITY CLASSIFICATION OF THIS PAGE

Unclassified 
10. SOURCE OF FUNDING NUMBERS (Continued).

DNA Task Code Y99QMXSB, Work Unit 00003, "Test Equipment and Techniques."

18. SUBJECT TERMS (Continued).

Clayey sand (SC)

Remolded specimens

Compression

Strain-path tests

Extension

Stress-path tests

Laboratory tests

Undisturbed specimens

\section{ABSTRACT (Continued).}

Test results proved that in all of the strain-path tests conducted on remolded specimens, a unique stress path was produced by following a given strain path at a single strain rate. However, because of the inherent nonhomogeneity of the undisturbed specimens, significant variability was observed in the data from those tests. Specimens that followed the SP3 strain path reached a point of continuing strain with little change in principal stress difference or mean normal stress; during this time the stress paths were in the proximity of the fallure envelope. With knowledge of this behavior, it was shown that the data from several tests, which followed a SP3 strain path and were conducted at different levels of prestress, would define a fallure envelope. Results from the reverse strain-path tests proved that a unique strain path was not obtained by following a given stress path; in this case, one generated by SP3B. The resulting strain magnitudes were dependent upon the length of time the stress path moved along the fallure envelope. The strain-rate tests showed a slight increase in peak stress difference with increasing strain rate.

In the stress-path test phase, wES conducted both compression and extension tests under constant axial and constant radial stress boundary conditions with its manually controlled pressure system. The available test data were insufficient to characterize the hardening response of the CARES material as either isotropic or kinematic. Several load-unload-reload tests were successfully conducted; the information gained from these tests was used for constitutive model fitting. 


\section{PREFACE}

The Geomechanics Division (GD), Structures Laboratory (SL), of the U.S. Army Engineer Waterways Experiment Station (WES) was funded by the Defense Nuclear Agency (DNA) to conduct a series of stress-path and strain-path tests on remolded and undisturbed specimens of CARES-Dry clayey sand. This research work was conducted during the period April 1984 through July 1984 and was funded under DNA Task Code Y99QMXSB, Work Unit 00003, "Test Equipment and Techniques."

The laboratory testing was performed and this report was prepared by Mr. Stephen A. Akers, GD, under the general direction of Dr. J. G. Jackson, Jr., Chief, GD, and Mr. J. Q. Ehrgott, Chief, Operations Group, GD. Mr. A. E. Jackson, Jr., provided technical assistance during the test program and during the preparation of this report. Numerous GD technicians and aides assisted in the testing program. This report was transmitted to the sponsor in September 1985.

COL Tilford C. Cree1, CE, and COL Robert C. Lee, CE, were the Commanders and Directors of WES during this investigation. COL Allen F. Grum, USA, was the previous Director and COL Dwayne G. Lee, CE, is the present Commander and Director. Mr. F. R. Brown and Dr. Robert W. Whalin were the WES Technical Directors. Mr. Bryant Mather was Chief, SL. 


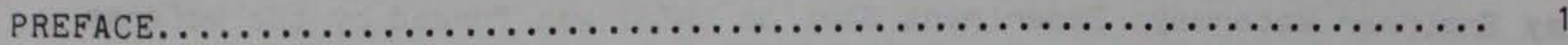

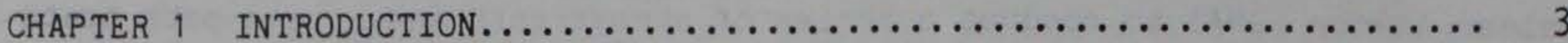

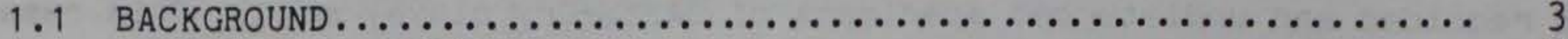

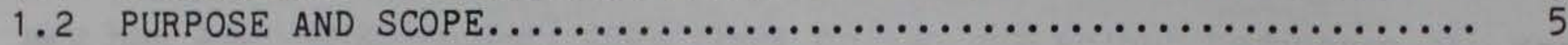

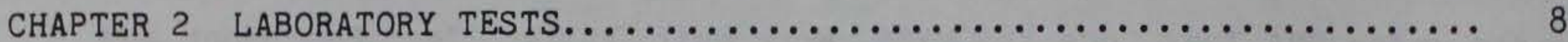

2.1 COMPOSITION AND INDEX PROPERTIES $\ldots \ldots \ldots \ldots \ldots \ldots \ldots \ldots \ldots \ldots \ldots \ldots$. 8

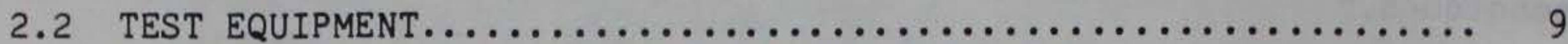

2.2.1 Test Chamber, Loader, and Pressure Console................ 9

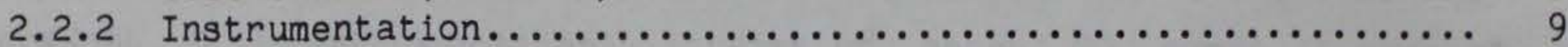

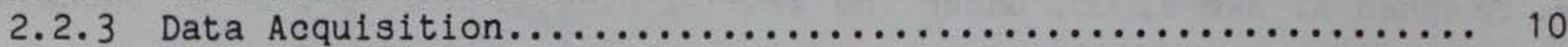

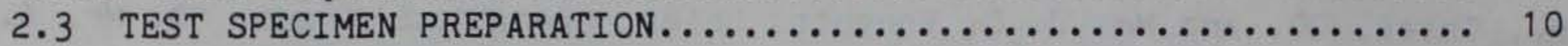

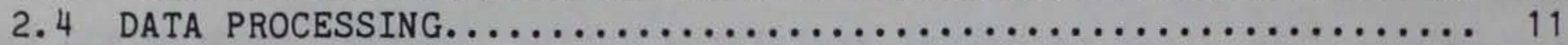

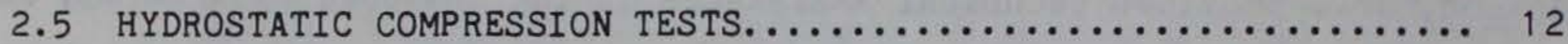

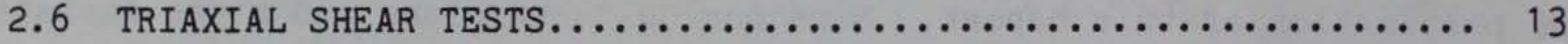

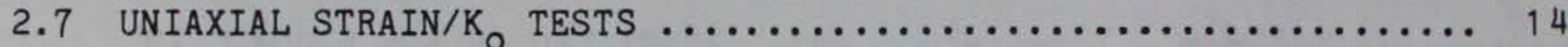

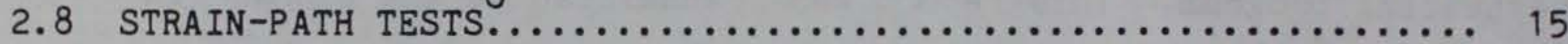

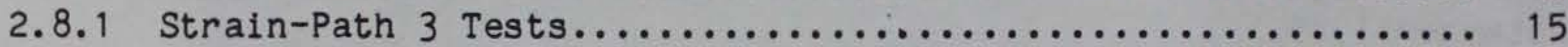

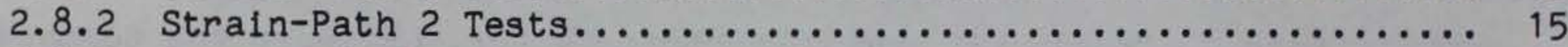

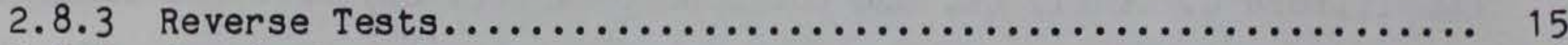

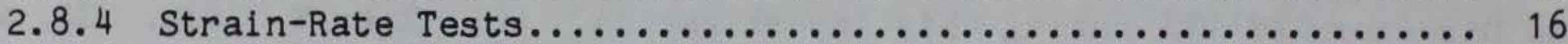

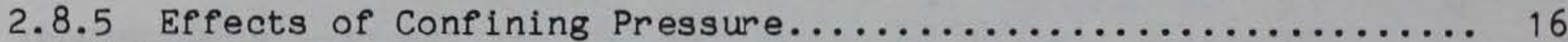

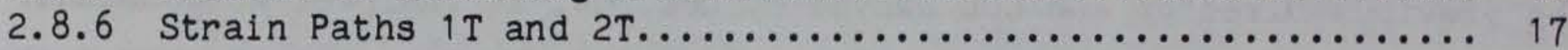

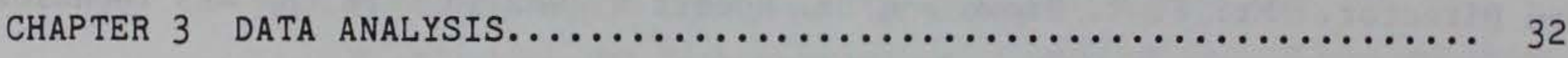

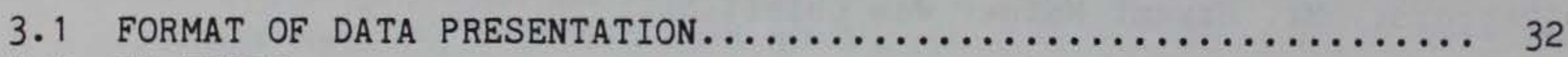

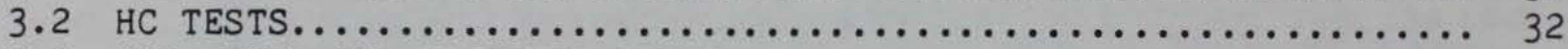

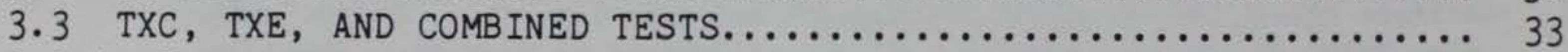

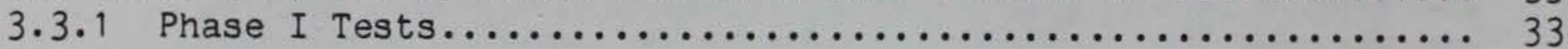

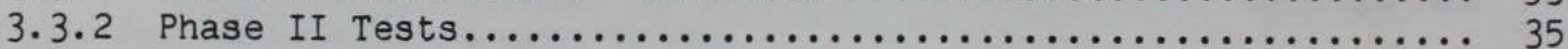

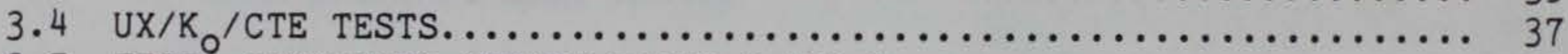

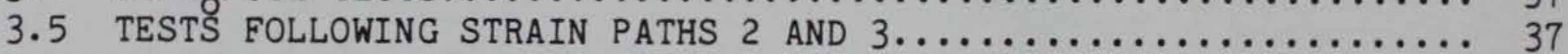

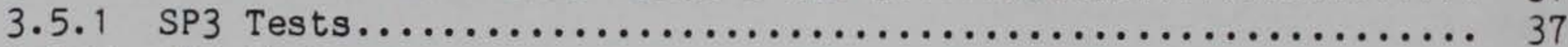

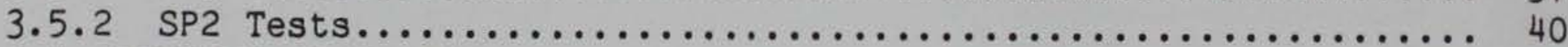

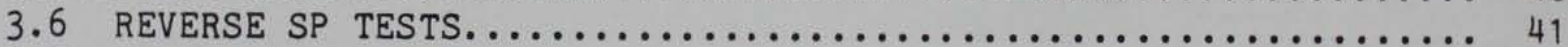

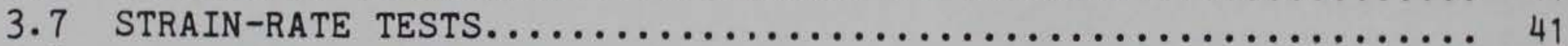

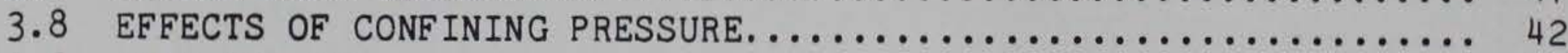

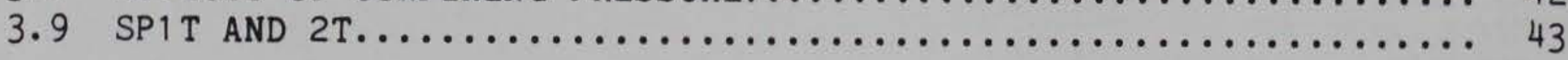

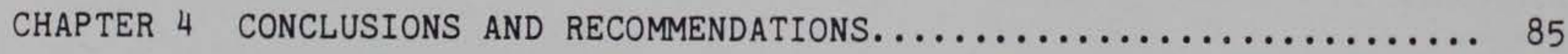

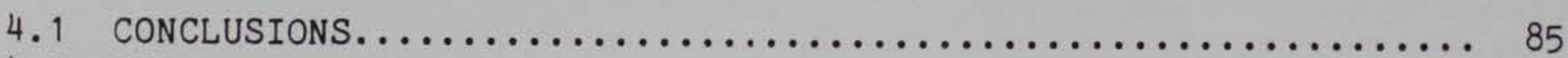

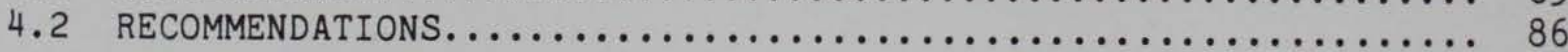

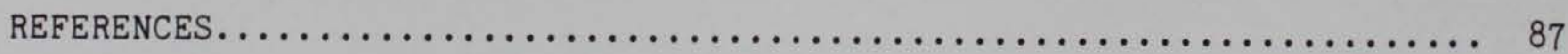

PLATES $1-75$ 


\section{AXISYMMETRIC STRAIN-PATH AND STRESS-PATH TESTS \\ ON CAREŚ-DRY CLAYEY SAND}

CHAPTER 1

INTRODUCTION

\subsection{BACKGROUND}

In FY 83, the US Army Engineer Waterways Experiment Station (WES) developed the test equipment and procedures necessary to conduct axisymmetric strain-path tests on cylindrical specimens of granular material. Strain-path tests were then conducted on remolded specimens of Nellis Baseline sand and undisturbed DIRECT COURSE specimens; the test results were documented in two reports (References 1 and 2, respectively). The Nellis Baseline strain-path investigation consisted of a two phase test program. The first phase was undertaken to determine whether strain-path tests could indeed be conducted with the WES manually controlled pressure system and whether the test data would be adequately repeatable. This phase consisted of hydrostatic compression (HC) tests, triaxial compression (TXC) tests, and strain-path tests following paths $2 \mathrm{~A}, 2 \mathrm{~B}, 2 \mathrm{C}, 3 \mathrm{~A}, 3 \mathrm{~B}$, and $3 \mathrm{C}$ (Figures 1.1 and 1.2). Data from these strain-path tests indicated that a unique stress path was produced when a given strain path was followed at a single strain rate. The second phase of the test program was conducted to determine whether a unique relationship between stress and strain existed. For this phase, reverse strain-path tests and strain-rate tests were performed. The reverse strain-path tests were conducted by following the stress path that had been measured in a previous strain-path test. The strain-rate tests were conducted by following a given strain path at different deformation rates. Data from the reverse strain-path tests indicated that the relationship between stress and strain for SP3B was not unique. In addition, the data illustrated that an infinite number of strain paths could be generated; the magnitudes of the resulting strains were a function of the length of time each stress path stayed in the proximity of the failure surface. The strain-rate tests exhibited a consistent increase in peak stress difference with increasing strain rate.

In the second FY 83 test program, tests following SP3A were conducted on undisturbed specimens from the DIRECT COURSE site. Results from these tests 
showed significant variability (more so than in the remolded data), even though the specimens were selected from approximately the same depth. This observation dramatized the fact that, in order to observe subtle changes in specimen response during strain-path tests, remolded specimens were needed.

WES'S FY 83 laboratory work coincided with strain-path testing at the University of Colorado (UC), Terra Tek, Inc. (TT), and the University of California at Los Angeles (UCLA). Strain-path tests at UC were conducted on remolded Nellis Baseline sand in a cubical triaxial shear (TX) device; this device permits the independent control of all three principal stresses (Reference 3). Strain-path tests were conducted at TT on remolded specimens of Nellis Baseline sand and on remolded and undisturbed specimens of CARES-Dry clayey sand. Strain-path tests were conducted at UCLA on undisturbed CARES specimens following strain paths $1 \mathrm{~T}$ and $2 \mathrm{~T}$ (Figures 1.1 and 1.2); results from these tests were reported in Reference 4. The strain-path tests at TT and UCLA were conducted in an axisymmetric configuration similar to that at WES. The WES FY 84 strain-path test program was conducted on remolded and undisturbed specimens of CARES-Dry clayey sand. The objectives of this program were to duplicate the FY 83 strain-path test matrix, investigate questions which arose from the FY 83 strain-path tests, and replicate the strainpath tests conducted at UCLA on undisturbed CARES specimens.

Coincident with the start of the strain-path testing, there was a need for specific mechanical response data for the CARES material in order to answer questions posed by material modelers. These questions were: (1) should the hardening response of the CARES material be modeled as isotropic or kinematic and (2) how does the material behave under several load-unload-reload cycles with each cycle extending into the compression and extension regimes of stress space? Triaxial extension (TXE) and special combined stress-path tests were designed and conducted in an attempt to answer the modelers' questions. As part of this work, several tests were also conducted to determine if more complicated stress-path tests could be conducted with the existing weS manually controlled pressure system. These tests were conducted with a uniaxial strain/K $K_{0}\left(U X / K_{0}\right)$ load-unload cycle to zero stress difference, followed by extension loading to failure under constant axial stress conditions. Finally, the FY 84 test program addressed a discrepancy between UX/Ko test data for the CARES material obtained from two different laboratories. 


\subsection{PURPOSE}

The purpose of this report is to document the test results obtained from the WES FY 84 laboratory investigation conducted on undisturbed and remolded specimens of CARES-Dry clayey sand. The test program was designed to measure the static mechanical response of the CARES material while following specific strain and stress paths. Test procedures and equipment used during testing are described in Chapter 2. Results from the laboratory test program are also presented and summarized in Chapter 2. A comprehensive analysis of the test results is presented in Chapter 3. Chapter 4 summarizes the main conclusions discussed in Chapter 3 and provides recommendations for further testing. 


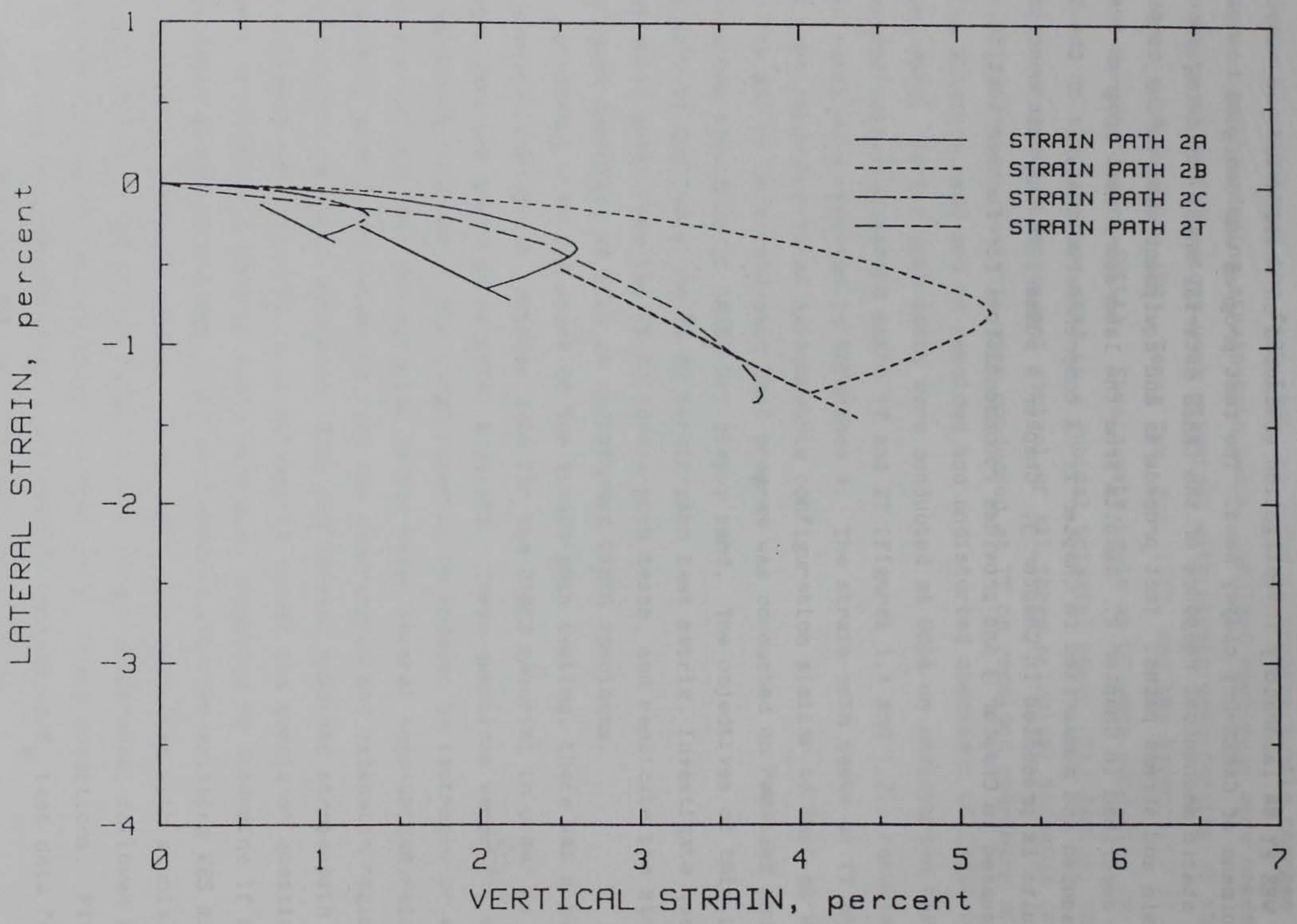

Figure 1.1. Comparison of strain paths $2 \mathrm{~A}, 2 \mathrm{~B}, 2 \mathrm{C}$ and $2 \mathrm{~T}$. 


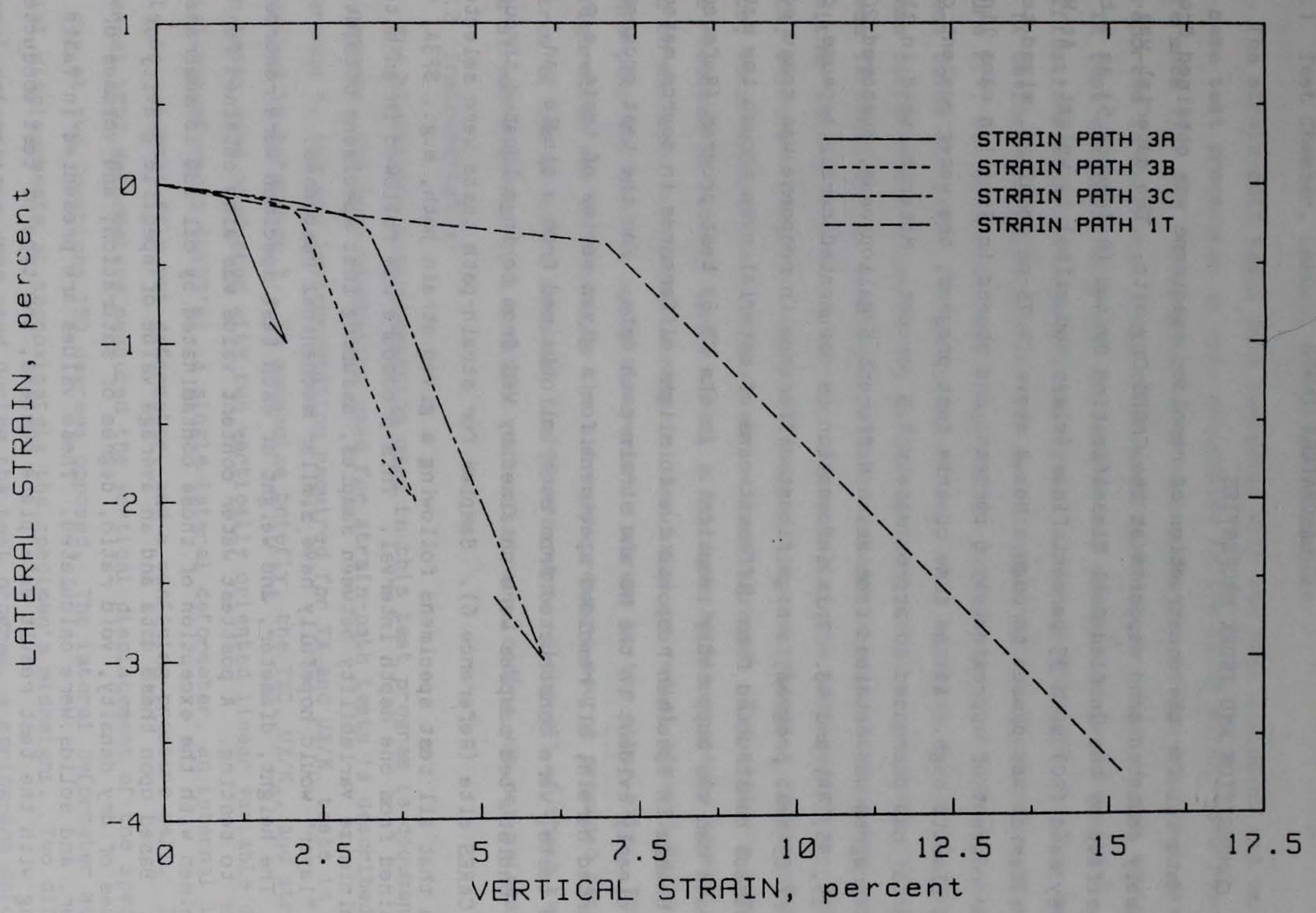

Figure 1.2. Comparison of strain paths $3 \mathrm{~A}, 3 \mathrm{~B}, 3 \mathrm{C}$ and $1 \mathrm{~T}$. 


\section{CHAPTER 2}

\section{LABORATORY TESTS}

\subsection{COMPOSITION AND INDEX PROPERTIES}

Material for the construction of remolded specimens was obtained from the variably cemented sand deposits at the CARES-Dry site. The material was classified by the Unified Soil Classification System (Reference 5) as a brown clayey sand (SC) with 33 percent fines (clays and silts) by weight. At WES, the material was passed through a No. 4 sieve (4.76-mm opening), mixed to a water content of approximately 6 percent, and stored in 5-gallon cans lined with plastic bags. At the time of this test program, the water content of the material had decreased to approximately 4.5 percent. Material used in this test program was obtained from seven different 5-gallon cans, numbered 20, 29, $32,35,36,44$, and 45 . This information is documented herein because, during the FY 83 test program, a significant difference in response was measured for specimens constructed from different cans of material even though the material in each can was supposedly identical. In the FY 83 test program (Reference 1), the change in specimen response due to slight differences in source material was clearly evident in the HC and strain-path data. For the test program reported herein, all remolded specimens for a given series of tests, e.g., all SP3B tests, were constructed from material obtained from a single can.

Undisturbed samples were obtained by WES from borings located throughout the CARES site (Reference 6). Samples for strain-path tests were selected such that all test specimens following a given strain path, e.g., SP3A, were obtained from one depth interval. This procedure was followed in an attempt to minimize variability between samples, assuming that specimens obtained from one "layer" would hopefully have similar mechanical responses.

The height, diameter, and weight of each test specimen were recorded prior to testing. A posttest water content value was also obtained for each specimen with the exception of those contaminated by oil due to membrane leakage. Based upon these data and an average value of specific gravity of 2.68 , values of dry density, void ratio, degree of saturation, and volumes of air, water, and solids were calculated. These values are presented in Table 2.1 along with the test result data plate numbers, the type of test conducted, and the can number from which material for remolded specimens was obtained. 


\subsection{TEST EQUIPMENT}

\subsubsection{Test Chamber, Loader, and Pressure Console}

The strain-path tests were conducted with the same equipment and using the same test procedures as were documented in the FY 83 strain-path report (Reference 1). The stress-path tests were conducted in the same test device as reported in Reference 1 but after modifications were made to the equipment. The TX test equipment and test procedures are documented in Reference 7 . The modifications mentioned above involved the use of an extension loading head in the TX device. In comparison to the conventional TXC loading system shown in Figure 2.1, the extension head (Figure 2.2) permits one to reduce the axial stress below the level of the radial stress, thus negative values of principal stress difference are achieved. Either constant axial or constant radial stress TX tests can be conducted with the extension head, loading the specimens in either compression or extension. For example, the combined stress-path tests, which extended into both the compression and extension regime of stress space, were conducted with the extension loading head. Two pressure supplies were required to conduct these tests; a nitrogen-bottle pressure source was used to maintain a constant stress (either axial or radial stress) while a 70-MPa pump was used to apply the variable stress to the other axis. This configuration was used because the nitrogen pressure source provided better control of the pressure than did the 70-MPa pump. Since all of these tests were stress controlled, a constant rate of strain loading was not maintained.

\subsubsection{Instrumentation}

The electronic instrumentation used in this test program is documented in two reports, the instrumentation used for strain-path tests is described in Reference 1 , and the instrumentation required for $T X$ and $U X / K_{0}$ tests is described in Reference 7 . To summarize briefly, the $T X C, U X / K_{0}$, and strainpath tests were conducted with two vertically oriented linear variable differential transformers (LVDT's), a single lateral deformeter, an internal load cell, an external film pot deformeter, and a confining pressure cell. The two internal LVDT deformeters measured the vertical displacement of the specimen top cap at two locations 180 degrees opposed. The lateral deformeter measured radial displacements at approximately the specimen's midheight. Two different lateral deformeters were used during the test program, a spring-arm deformeter to measure displacements during tests with large radial strains, e.g., HC and 
TX tests, and an LVDT deformeter to accurately measure the smaller radial strains which developed in the strain-path and UX/Ko tests. The TXE and the combined stress-path tests were conducted with a single internal film pot deformeter, a single lateral deformeter, and two pressure cells (one to measure axial stress and the other to measure confining pressure). Different lateral deformeters were required for the two different specimen sizes, i.e., 5- and 7.6-cm-diameter specimens.

\subsubsection{Data Acquisition}

During a test, analog signals from the electronic instrumentation were measured at specific time intervals using a programmable data acquisition system. The data were converted to appropriate values of stress and strain and stored on magnetic tape for subsequent data processing. As an option, the recorded stress and strain data could be output to an $X-Y$ plotter in the form of stress paths and strain paths. This option helped the engineer follow a predetermined stress or strain path. The predetermined path was plotted on the $X-Y$ plotter prior to conducting the test; then the engineer attempted to adjust the stresses during the test so that the measured stresses or strains plotted atop the desired path.

\subsection{TEST SPECIMEN PREPARATION}

Remolded test specimens were constructed by jacking the CARES material into 5- or 7.6-cm-diameter steel molds. The material was placed in five lifts of equal height for both the strain-path and TX specimens. With this process, each 1 ift should have the same wet density as the overall specimen, which was nominally $1.9 \mathrm{~g} / \mathrm{cc}$. An odd number of 1 ifts was used in specimen construction to avoid a lift interface at the specimen midheight. Remolded specimens are identified in this report by the prefix "RDC" for remolded Dry CARES. The test number is then the prefix "RDC" followed by a two-digit number generated in sequential order during the test program.

Undisturbed test specimens, identified in this report by the prefix "UDC" for undisturbed Dry CARES, were cut from undisturbed core samples that had been wrapped in aluminum foil, placed in cardboard tubes, then sealed in the field with wax. Table 2.2 identifies the site, the boring number, and the depth (in meters) of each undisturbed specimen, e.g., specimen UDC73 was from the Dry CARES (DCAR) site, boring 38, and 29.44-meter depth, or DCAR38-29.44. Each undisturbed test specimen was frozen for a minimum of 24 hours before 
being prepared for testing. The cardboard and foil were cut fram the frozen specimen and the procedures outlinied below were followed.

After specimen height, diameter, and weight measurements were recorded, two rubber membranes were placed on the specimen, and the outer membrane was covered with a synthetic rubber; this coating protected the membrane fram the deteriorating effects of the hydraulic oil. All tests were conducted in an undrained manner, i.e., no pore fluid (air or water) was allowed to drain from the membrane-enclosed specimen.

Prior to conducting TXE tests, one of three base pedestals was selected for use based upon the type of test to be conducted and the magnitude of the anticipated strains. These three different base pedestals were needed because the loading piston within the extension head has a limited range of vertical displacement. Thus, in order to achieve vertical strains of 15 percent or greater, the initial position of the loading piston within the extension head had to be carefully set.

\subsection{DATA PROCESSING}

Data processing procedures for the strain-path tests are documented in Reference 1; procedures for the TX tests are documented in Reference 7 . Two important assumptions which were made in the data processing are outlined below.

Volumetric strains were calculated from deformeter measurements by assuming either a uniform-cylinder or truncated-cone deformed specimen shape. The uniform-cylinder approximation assumes that the specimen deforms as a right circular cylinder; the current diameter of the specimen, i.e., the original diameter minus the change in diameter, is assumed to exist over the entire length of the specimen. The truncated-cone approximation assumes that the current diameter is measured at the specimen's midheight and that the diameter changes linearly to the original pretest diameter at the ends of the specimen. All second-order terms were included in the calculations of volumetric strain. The uniform-shape assumption approximates the true volumetric strains more accurately during $\mathrm{HC}$ and at small axial strains during shear. The truncatedcone assumption approximates the true volumetric strains more accurately during shear at 1 arger axial strains, e.g., >7-8 percent. The true volumetric strains are typically somewhere between these two calculated values. 
When tests are conducted with the extension loading head, the axial stress must be corrected for the force of the confining pressure pushing up on the loading piston. A correction is applied such that the vertical load is equal to (a) the applied vertical pressure times the piston area minus (b) the confining pressure times the difference between the area of the loading piston and the area of the specimen. This correction is only applied when the area of the specimen is less than the area of the piston.

Individual strain-path and TX test results are presented on data plates using two different plotting formats. The TX test data are presented on fourcorner plots, similar to that shown in Figure 2.3, as principal stress difference versus mean normal stress (plot a), principal stress difference versus principal strain difference and axial strain (plot b), volumetric strain versus principal strain difference (plot $c$ ), and volumetric strain versus mean normal stress (plot d). The strain-path test data are presented on a $f i v e-$ plot $\mathrm{f}$ igure like that shown in Figure 2.4. The four plots (a-d) used on the four-corner plot are included along with an added plot of the intended and measured strain paths (plot e in Figure 2.4). With one exception, all stresses and strains are plotted from a pretest zero stress-zero strain state; thus, all plots include the strains measured during HC loading. The one exception is the strains in the strain-path plots (lateral strain versus vertical strain); these strains were rezeroed at the start of the strain-path loading.

\subsection{HYDROSTATIC COMPRESSION TESTS}

During an HC test, a uniform fluid loading is applied to the specimen, and the resulting deformations are measured at the specimen top and midheight. Four channels of data are recorded, two axial displacements, one radial displacement, and confining pressure. From these measurements, one can calculate axial strain (typically taken as the average of the two vertical LVDT deformeters), radial strain, mean normal stress, and volumetric strain. The effects of membrane deformations are assumed insignificant in the calculation of lateral strain.

HC tests were conducted in this test program for two purposes, first, to measure the bulk compressibility of the CARES material under an isotropic stress and, second, to ascertain the consistency or lack thereof between each can of material used for remolded specimens. Six high-pressure HC tests 
(RDC01, 02, 03, 19, 34, and 81) were conducted on specimens from four different cans of CARES material. Results from these six HC tests are presented in Plates $1-6$ as plots of mean normal stress versus axial strain, radial strain, and volumetric strain (both uniform and cone assumptions). In addition to these data, the HC loading of each strain-path and TX test specimen is available; a majority of these tests were loaded to a confining pressure of $6.9 \mathrm{MPa}$. Table 2.2 presents a summary of problems encountered, observations made, and thought processes invol ved in conducting these tests. This table lists the test number and data plate numbers, identifies the type of test conducted, and provides pertinent test notes for each of the 81 prepared test specimens.

\subsection{TRIAXIAL SHEAR TESTS}

Reference 7 describes the test procedures which were used in the FY 84 laboratory test program. Six channels of data were typically recorded in the conventional TXC tests and four channels in the TXE tests.

In the first phase of the stress-path test program, two TXC tests (RDCO 4 and 05 ) were conducted to determine the effects of specimen size, i.e., 5versus $7.6-\mathrm{cm}$-diameter specimens, on the measured response. A third TXC test (RDC23) was conducted for the purpose of analyzing the vertical and lateral strains during the initial loading. Results from these tests are presented in Plates 7-9.

Six TXE tests were conducted by imposing constant radial stress conditions on test specimens RDCO6 and 07 and constant axial stress conditions on test specimens RDC13-16. (Note that during tests RDC13 and 14 constant axial stress conditions were not maintained.) These six tests were performed in order to gain experience in conducting TXE tests and to supplement the existing extension data base. Results from these tests are presented in Plates 10-15.

One phase of the stress-path test program was to determine whether the CARES material exhibited characteristics of kinematic or isotropic hardening. In an attempt to characterize the hardening behavior, tests were conducted using the extension device. An initial loading of either compression or extension was applied, then the specimens were loaded in both compression and extension. These tests were the first set of combined stress path tests. The following labels have been used herein to identify the imposed stress paths: reduced triaxial compression (RTC) tests were loaded in compression under 
constant axial stress boundary conditions; conventional triaxial compression (CTC) tests were loaded in compression under constant radial stress boundary conditions and are often labeled as TXC tests; reduced triaxial extension (RTE) tests were loaded in extension under constant radial stress boundary conditions; conventional triaxial extension (CTE) tests were loaded in extension under constant axial stress boundary conditions. The combined stresspath tests have been identified with the appropriate extension and compression stress-path labels, i.e., a CTC/RTE test was loaded in compression first, then into extension, under constant radial stress boundary conditions. Tests RDC9-12 were conducted under constant radial stress boundary conditions, and tests RDC17 and 18 were conducted under constant axial stress boundary conditions. Results from these tests are presented in Plates 16-21.

Another phase of the stress-path test program was designed to measure the response of the CARES material under several different load-unload-reload conditions by extending the stress paths into both the compression and extension regions of stress space. All of these tests were conducted under constant radial stress conditions. During the loading sequence, each specimen was deformed to specific values of axial strain. In compression, the specimens were deformed to 5, 10, and 15 percent axial strain and then just a few percent strain on the final loading in compression. In extension, the specimens were loaded to various levels of axial strain; the intended extension strains for each test are summarized in Table 2.2. Results from these tests are presented in Plates 22-26.

\subsection{UNIAXIAL STRAIN/K。 TESTS}

Reference 7 describes the test procedures used to conduct the $U X / K_{0}$ tests. In these tests, the engineer observed a readout of 1 ateral deformation on a digital monitor and then manually adjusted the confining pressure in order to maintain a zero lateral strain condition. A constant axial strain rate was typically used throughout the loading phase. One significant difference between these specimens and other FY 84 test specimens was specimen length. Specimens for $U X / K_{0}$ tests had length-to-diameter (L/D) ratios of about 1; all other test specimens had L/D ratios of between 2 and 2.5 .

Three $U X / K_{0}$ tests (RDC50-52) were conducted as a check on response data furnished to WES by another laboratory. The three tests were conducted on specimens constructed with two different water contents (see Table 2.1), and 
were loaded at two different deformation rates. The data from these three tests are presented in Plates 27-29.

$\mathrm{K}_{\mathrm{O}} / \mathrm{CTE}$ tests were conducted to measure specimen response to a loading sequence commonly encountered during high-explosive field testing. Two specimens (RDC46 and 47) were loaded under $\mathrm{K}_{0}$ conditions, unloaded under $\mathrm{K}_{0}$ conditions to a zero stress difference state, then loaded to failure in extension under constant axial stress conditions. These tests were more difficult to conduct than the standard $U X / K_{0}$ tests because the vertical load was applied by a stress-controlled loading device as opposed to the normally used straincontrolled device. Results from these two tests are presented in Plates 30 and 31 .

\subsection{STRAIN-PATH TESTS}

The test procedures followed in this phase of the test program are described in the FY 83 strain-path report (Reference 1). One modification was made to the FY 83 procedures; the load-unload cycle during the HC prestress loading was not imposed upon the FY 84 test specimens.

\subsubsection{Strain-Path 3 Tests}

Ten strain-path tests were successfully conducted on 5-cm-diameter specimens of remolded CARES material following strain paths $3 \mathrm{~A}, 3 \mathrm{~B}$, and $3 \mathrm{C}$ (see Figure 1.2). Results from these 10 tests are presented in Plates 32-41. Six undisturbed CARES specimens $(7.2-\mathrm{cm}$ in diameter) were successfully tested following strain paths $3 \mathrm{~A}$ and $3 \mathrm{C}$. Data from these tests are summarized in Plates 42-47. An attempt was made to select all of the undisturbed specimens for each test series from one depth interval; this hopefully eliminated as much as possible the depth variation in the response of the in situ material.

\subsubsection{Strain-Path 2 Tests}

Ten strain-path tests were successfully conducted on 5-cm-diameter specimens of remolded CARES material following strain paths $2 \mathrm{~A}, 2 \mathrm{~B}$, and $2 \mathrm{C}$ (Figure 1.1); results are presented in Plates 48-57. No undisturbed specimens were tested along these strain paths.

\subsubsection{Reverse Tests}

These tests were conducted to determine whether a unique relationship existed between stress and strain. In the FY 83 test program, a unique stress 
path was measured while following a given strain path at a single strain rate. However, when this process was reversed, that is, when a stress path was followed and a strain path measured, several different strain paths were produced. The magnitude of the strains was a function of the length of time the stress path was in the proximity of the failure envelope; larger strains were recorded as the time near the failure envelope increased.

A single test (RDC62) was first conducted as a regular strain-path test following SP3B. The stress path from this test was digitized and followed in subsequent reverse tests. The axial deformation rate was kept the same in all of the reverse tests; the only difference between the tests was the rate at which the confining pressure was changed during the time the stress path followed the failure surface. Three reverse tests were conducted (RDC63, 64, and 67) with times along the failure surface of $4.6,1.2$, and 9.9 minutes, respectively. Results from these three reverse tests are presented in $\mathrm{Pl}$ ates $58-60$.

\subsubsection{Strain-Rate Tests}

Four strain-path tests (RDC65, 66, 68, and 72) were conducted on remolded specimens of CARES material following SP3B using three different deformation rates. A constant deformation rate up to the point of strain reversal (peak vertical strain) was used. Test RDC62 was used to provide a standard time (approximately 30 minutes) for comparison purposes. Three of the four tests were conducted in $17.6,5.3$, and 5.2 minutes; the fourth test was terminated at approximately the point of peak stress difference. Results from these four strain-rate tests are presented in Plates 61-64.

\subsubsection{Effects of Confining Pressure}

In this study and in the FY 83 test program, the stress paths produced by tests following any of the No. 3 strain paths also followed the failure envelope during some point in the tests. This was clearly evident from the stress-strain curves, i.e., the test reached a point after which increasing axial strains occurred with little change in either stress difference or mean normal stress. It seemed logical that a series of strain-path tests following one of the No. 3 strain paths should produce a well-def ined failure envelope if the tests were conducted at several different initial confining pressures. This assumption was investigated by conducting three strain-path tests (RDC69, 70, and 71) following SP3B with initial confining pressures of $3.45,1.72$, and 
$0.41 \mathrm{MPa}$, respectively, and by using the data from specimen RDC62 as a fourth test at $6.9 \mathrm{MPa}$. Results from tests RDC69, 70, and 71 are presented in Plates 65-67.

\subsubsection{Strain Paths $1 \mathrm{~T}$ and $2 \mathrm{~T}$}

Two strain paths, identified herein as strain paths $1 \mathrm{~T}$ and $2 \mathrm{~T}$ (see Figure 1.1 and 1.2), were followed by Dr. Poul Lade of UCLA when he conducted strain-path tests on undisturbed specimens of CARES material (Reference 4 ). In the WES test program, eight tests were conducted following these two paths. Three tests (UDC73, 74, and 80) followed strain path $1 \mathrm{~T}$ with an initial confining pressure of $0.41 \mathrm{MPa}$, and five tests followed strain path $2 \mathrm{~T}$ after prestress levels of 0.41 (UDC75, 76, and 77) and $3.45 \mathrm{MPa}$ (UDC78 and 79) were imposed. The results from these eight tests are presented in Plates 68-75. Two of the specimens tested in this series (UDC75 and 78) had unusually high water content values of 8.9 and 8.5 percent, respectively. No reason could be found for these specimens to have such high values. However, the high water contents had a clear effect upon the mechanical properties of the specimens, i.e., peak values of stress difference were very low in comparison to their companion tests, and the specimens exhibited very large volumetric strains during HC loading. 
Table 2.1. Test specimen composition properties.

\begin{tabular}{|c|c|c|c|c|c|c|c|c|c|c|c|}
\hline $\begin{array}{r}\text { Test } \\
\text { No. }\end{array}$ & $\begin{array}{c}\text { Plate } \\
\text { No. } \\
\end{array}$ & $\begin{array}{c}\text { Type of } \\
\text { Test* } \\
\end{array}$ & $\begin{array}{l}\text { Can } \\
\text { No. } \\
\end{array}$ & $\begin{array}{l}\text { Wet } \\
\text { Density } \\
\gamma, \mathrm{g} / \mathrm{cc} \\
\end{array}$ & $\begin{array}{c}\text { Water } \\
\text { Content } \\
\text { W, \% } \\
\end{array}$ & $\begin{array}{c}\text { Dry } \\
\text { Density } \\
\gamma_{d}, g / c c \\
\end{array}$ & $\begin{array}{l}\text { Void } \\
\text { Ratio } \\
\text { e }\end{array}$ & $\begin{array}{l}\text { Degree of } \\
\text { Saturation } \\
\text { S, \% }\end{array}$ & $\begin{array}{l}\frac{V}{A i r} \\
V_{a}, \% \\
\end{array}$ & $\begin{array}{l}\text { olume of } \\
\text { Solids } \\
v_{s}, \% \\
\end{array}$ & $\begin{array}{l}\text { Water } \\
V_{\text {W }} \text { \% } \\
\end{array}$ \\
\hline $\mathrm{RDC} 01$ & 1 & $\mathrm{HC}$ & 20 & 1.859 & 4.58 & 1.778 & 0.508 & 24.2 & 25.5 & 66.3 & 8.1 \\
\hline $\mathrm{RDCO} 2$ & 2 & $\mathrm{HC}$ & 20 & 1.865 & 4.60 & 1.783 & 0.503 & 24.5 & 25.3 & 66.5 & 8.2 \\
\hline $\mathrm{RDCO} 3$ & 3 & $\mathrm{HC}$ & 20 & 1.894 & 4.66 & 1.810 & 0.481 & 26.0 & 24.0 & 67.5 & 8.4 \\
\hline $\mathrm{RDCO} 4$ & 7 & CTC & 20 & 1.896 & 4.59 & 1.813 & 0.478 & 25.7 & 24.0 & 67.6 & 8.3 \\
\hline RDC05 & 8 & CTC & 20 & 1.880 & 4.60 & 1.797 & 0.491 & 25.1 & 24.7 & 67.1 & 8.3 \\
\hline RDC06 & 10 & RTE & 20 & 1.892 & 4.48 & 1.811 & 0.480 & 25.0 & 24.3 & 67.6 & 8.1 \\
\hline $\mathrm{RDC} 07$ & 11 & RTE & 20 & 1.889 & 4.48 & 1.808 & 0.482 & 24.9 & 24.4 & 67.5 & 8.1 \\
\hline $\mathrm{RDC} 08$ & -- & RTE & 20 & 1.895 & $t \dagger$ & -- & -- & -- & -- & -- & $\because$ \\
\hline RDCO9 & 16 & CTC/RTE & 20 & 1.895 & $t \dagger$ & -- & -- & -- & -- & -- & -- \\
\hline $\mathrm{RDC} 10$ & 17 & CTC/RTE & 29 & 1.896 & $t+$ & -- & -- & -- & -- & -- & -- \\
\hline RDC 11 & 18 & $\mathrm{RTE} / \mathrm{CTC}$ & 29 & 1.893 & 4.31 & 1.815 & 0.477 & 24.2 & 24.5 & 67.7 & 7.8 \\
\hline $\mathrm{RDC} 12$ & 19 & $\mathrm{RTE} / \mathrm{CTC}$ & 29 & 1.897 & $t t$ & -- & -- & -- & -- & -- & -- \\
\hline $\mathrm{RDC} 13$ & 12 & СТE & -- & 1.901 & $t+$ & -- & -- & -- & -- & -- & -- \\
\hline $\mathrm{RDC} 14$ & 13 & CTE & 29 & 1.900 & $t t$ & -- & -- & -- & -- & -- & -- \\
\hline $\mathrm{RDC} 15$ & 14 & CTE & 32 & 1.900 & $t t$ & -- & -- & -- & -- & -- & -- \\
\hline $\mathrm{RDC} 16$ & 15 & CTE & 32 & 1.894 & $t+$ & -- & -- & -- & - & -- & -- \\
\hline $\mathrm{RDC} 17$ & 20 & $\mathrm{RTC} / \mathrm{CTE}$ & 32 & 1.892 & tt & -- & -- & -- & -- & -- & -- \\
\hline $\mathrm{RDC} 18$ & 21 & $\mathrm{RTC} / \mathrm{CTE}$ & 32 & 1.893 & $t \dagger$ & -- & -- & -- & -- & -- & -- \\
\hline $\mathrm{RDC} 19$ & 4 & $\mathrm{HC}$ & 32 & 1.892 & 4.90 & 1.804 & 0.486 & 27.0 & 23.9 & 67.3 & 8.8 \\
\hline $\mathrm{RDC} 20$ & 32 & SP3A & 32 & 1.899 & 4.81 & 1.812 & 0.479 & 26.9 & 23.7 & 67.6 & 8.7 \\
\hline $\mathrm{RDC} 21$ & 33 & SP3A & 32 & 1.894 & 4.83 & 1.807 & 0.483 & 26.8 & 23.9 & 67.4 & 8.7 \\
\hline RDC22 & 34 & SP3A & 32 & 1.901 & 4.80 & 1.814 & 0.477 & 26.9 & 23.6 & 67.7 & 8.7 \\
\hline
\end{tabular}


Table 2.1. (Conti nued).

\begin{tabular}{|c|c|c|c|c|c|c|c|c|c|c|c|}
\hline \multirow[b]{2}{*}{$\begin{array}{r}\text { Test } \\
\text { No. } \\
\end{array}$} & \multirow[b]{2}{*}{$\begin{array}{l}\text { Plate } \\
\text { No. }\end{array}$} & \multirow[b]{2}{*}{$\begin{array}{l}\text { Type of } \\
\text { Test* }\end{array}$} & \multirow[b]{2}{*}{$\begin{array}{l}\text { Can } \\
\text { No. } \\
\end{array}$} & \multirow{2}{*}{$\begin{array}{l}\text { Wet } \\
\text { Density } \\
\gamma, \mathrm{g} / \mathrm{cc} \\
\end{array}$} & \multirow{2}{*}{$\begin{array}{c}\text { Water } \\
\text { Content } \\
\text { w, \% } \\
\end{array}$} & \multirow{2}{*}{$\begin{array}{c}\text { Dry } \\
\text { Density } \\
\gamma_{d}, g / c c \\
\end{array}$} & \multirow{2}{*}{$\begin{array}{c}\text { Void } \\
\text { Ratio } \\
\text { e } \\
\end{array}$} & \multirow{2}{*}{$\begin{array}{c}\text { Degree of } \\
\text { Saturation } \\
\text { S,\% }\end{array}$} & \multicolumn{3}{|c|}{ Volume of } \\
\hline & & & & & & & & & $\begin{array}{c}\text { Air } \\
\mathrm{v}_{\mathrm{a}}, \% \\
\end{array}$ & $\begin{array}{l}\text { Solids } \\
\mathrm{v}_{\mathrm{s}}, \% \\
\end{array}$ & $\begin{array}{l}\text { Water } \\
\mathrm{V}_{\mathrm{w}}, \% \\
\end{array}$ \\
\hline $\mathrm{RDC} 23$ & 9 & CTC & 32 & 1.910 & 4.69 & 1.824 & 0.469 & 26.8 & 23.4 & 68.1 & 8.5 \\
\hline RDC24 & 51 & SP2B & 36 & 1.902 & 4.09 & 1.827 & 0.467 & 23.5 & 24.3 & 68.2 & 7.5 \\
\hline RDC25 & 52 & SP2B & 36 & 1.894 & 4.08 & 1.820 & 0.473 & 23.1 & 24.7 & 67.9 & 7.4 \\
\hline $\mathrm{RDC} 26$ & 53 & SP2B & 36 & 1.886 & 3.99 & 1.814 & 0.478 & 22.4 & 25.1 & 67.7 & 7.2 \\
\hline $\mathrm{RDC} 27$ & 54 & SP2B & 36 & 1.901 & $t+$ & -- & -- & -- & -- & -- & -- \\
\hline $\mathrm{RDC} 28$ & 35 & SP3B & 36 & 1.903 & 3.96 & 1.831 & 0.464 & 22.9 & 24.4 & 68.3 & 7.2 \\
\hline RDC29 & 36 & SP3B & 36 & 1.897 & 3.98 & 1.824 & 0.469 & 22.7 & 24.7 & 68.1 & 7.3 \\
\hline $\mathrm{RDC} 30$ & 37 & SP3B & 36 & 1.902 & 4.06 & 1.828 & 0.466 & 23.3 & 24.4 & 68.2 & 7.4 \\
\hline $\mathrm{RDC} 31$ & 39 & $\mathrm{SP} 3 \mathrm{C}$ & 36 & 1.895 & 4.02 & 1.822 & 0.471 & 22.9 & 24.7 & 68.0 & 7.3 \\
\hline RDC32 & 40 & SP3C & 36 & 1.892 & 3.97 & 1.820 & 0.473 & 22.5 & 24.9 & 67.9 & 7.2 \\
\hline RDC33 & 41 & SP3C & 36 & 1.901 & 4.02 & 1.828 & 0.466 & 23.1 & 24.5 & 68.2 & 7.3 \\
\hline $\mathrm{RDC} 34$ & 5 & $\mathrm{HC}$ & 36 & 1.904 & 4.01 & $1.831^{\circ}$ & 0.464 & 23.2 & 24.4 & 68.3 & 7.3 \\
\hline $\mathrm{RDC} 35$ & 48 & $\mathrm{SP} 2 \mathrm{~A}$ & 44 & 1.904 & 4.01 & 1.831 & 0.464 & 23.2 & 24.4 & 68.3 & 7.3 \\
\hline $\mathrm{RDC} 36$ & 49 & $\mathrm{SP} 2 \mathrm{~A}$ & 44 & 1.906 & 3.83 & 1.836 & 0.460 & 22.3 & 24.5 & 68.5 & 7.0 \\
\hline $\mathrm{RDC} 37$ & 50 & $\mathrm{SP} 2 \mathrm{~A}$ & 44 & 1.899 & 3.82 & 1.829 & 0.465 & 22.0 & 24.8 & 68.3 & 7.0 \\
\hline RDC38 & 55 & $\mathrm{SP} 2 \mathrm{C}$ & 44 & 1.903 & 3.94 & 1.831 & 0.464 & 22.8 & 24.5 & 68.3 & 7.2 \\
\hline RDC39 & 22 & CTC/RTE & 44 & 1.899 & tt & -- & -- & -- & -- & -- & -- \\
\hline $\mathrm{RDC} 40$ & 23 & CTC/RTE & 44 & 1.899 & $t t$ & -- & -- & -- & -- & -- & -- \\
\hline $\mathrm{RDC} 41$ & -- & CTC/RTE & 44 & 1.893 & $t t$ & - & -- & -- & -- & -- & -- \\
\hline $\mathrm{RDC} 42$ & 24 & $\mathrm{CTC} / \mathrm{RTE}$ & 44 & 1.894 & $t t$ & -- & -- & -- & -- & - & -- \\
\hline $\mathrm{RDC} 43$ & 25 & CTC/RTE & 44 & 1.894 & $t t$ & - & -- & -- & -- & -- & -- \\
\hline $\mathrm{RDC} 44$ & -- & CTC/RTE & 44 & 1.892 & $t+$ & -- & -- & -- & -- & -- & -- \\
\hline
\end{tabular}


Table 2.1. (Continued).

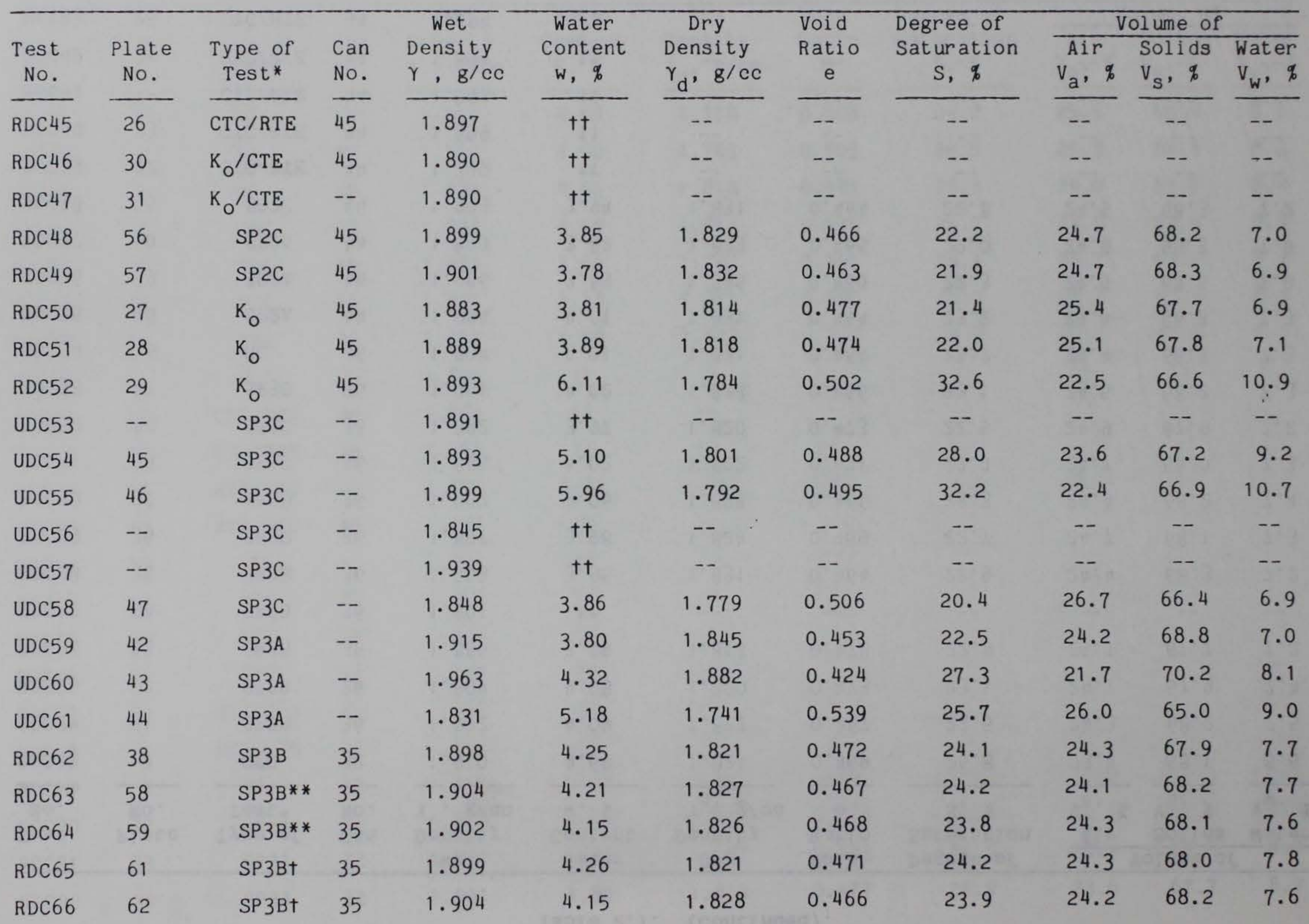


Table 2.1. (Concluded).

\begin{tabular}{|c|c|c|c|c|c|c|c|c|c|c|c|}
\hline \multirow[b]{2}{*}{$\begin{array}{c}\text { Test } \\
\text { No. }\end{array}$} & \multirow[b]{2}{*}{$\begin{array}{c}\text { Plate } \\
\text { No. }\end{array}$} & \multirow[b]{2}{*}{$\begin{array}{c}\text { Type of } \\
\text { Test* } \\
\end{array}$} & \multirow[b]{2}{*}{$\begin{array}{l}\text { Can } \\
\text { No. } \\
\end{array}$} & & \multirow{2}{*}{$\begin{array}{c}\text { Water } \\
\text { Content } \\
\text { w, \% }\end{array}$} & \multirow{2}{*}{\begin{tabular}{l}
\multicolumn{1}{c}{ Dry } \\
Density \\
$\gamma_{d}, g / c c$ \\
\end{tabular}} & \multirow{2}{*}{$\begin{array}{l}\text { Void } \\
\text { Ratio } \\
\text { e } \\
\end{array}$} & \multirow{2}{*}{$\begin{array}{c}\text { Degree of } \\
\text { Saturation } \\
\text { S, \% } \\
\end{array}$} & \multicolumn{3}{|c|}{ Volume of } \\
\hline & & & & $\begin{array}{l}\text { Density } \\
\gamma, \mathrm{g} / \mathrm{cc} \\
\end{array}$ & & & & & $\begin{array}{l}\text { Air } \\
v_{a}, \% \\
\end{array}$ & $\begin{array}{l}\text { Solids } \\
\mathrm{v}_{\mathrm{s}}, \% \\
\end{array}$ & $\begin{array}{l}\text { Water } \\
\mathrm{v}_{\mathrm{w}}, \% \\
\end{array}$ \\
\hline RDC67 & 60 & SP3B** & 35 & 1.904 & 4.24 & 1.827 & 0.467 & 24.3 & 24.1 & 68.2 & 7.7 \\
\hline RDC68 & 63 & SP3Bt & 35 & 1.902 & 4.11 & 1.827 & 0.467 & 23.6 & 24.3 & 68.2 & 7.5 \\
\hline RDC69 & 65 & SP3B & 35 & 1.901 & 4.14 & 1.825 & 0.468 & 23.7 & 24.3 & 68.1 & 7.6 \\
\hline RDC70 & 66 & SP3B & 35 & 1.902 & 4.13 & 1.827 & 0.467 & 23.7 & 24.3 & 68.2 & 7.5 \\
\hline RDC71 & 67 & SP3B & 35 & 1.906 & 4.13 & 1.830 & 0.464 & 23.8 & 24.1 & 68.3 & 7.6 \\
\hline $\mathrm{RDC72}$ & 64 & SP3Bt & 35 & 1.903 & 4.11 & 1.828 & 0.466 & 23.6 & 24.3 & 68.2 & 7.5 \\
\hline RDC73 & 68 & SP1T & -- & 1.805 & $t+$ & -- & -- & -- & -- & -- & -- \\
\hline UDC7 4 & 69 & $\mathrm{SP} 1 \mathrm{~T}$ & -- & 1.860 & 2.29 & 1.818 & 0.474 & 13.0 & 28.0 & 67.8 & 4.2 \\
\hline UDC75 & 71 & SP2T & -- & 1.993 & 8.89 & 1.830 & 0.464 & 51.3 & 15.4 & 68.3 & 16.3 \\
\hline UDC76 & 72 & SP2T & -- & 1.763 & 2.62 & 1.718 & 0.560 & 12.5 & 31.4 & 64.1 & 4.5 \\
\hline UDC77 & 73 & SP2T & -- & 1.865 & 2.63 & 1.817 & 0.475 & 14.8 & 27.4 & 67.8 & 4.8 \\
\hline UDC7 8 & 74 & SP2T & -- & 1.878 & 8.50 & 1.731 & 0.548 & 41.5 & 20.7 & 64.6 & 14.7 \\
\hline UDC79 & 75 & SP2T & -- & 1.729 & 3.77 & 1.666 & 0.608 & 16.6 & 31.5 & 62.2 & 6.3 \\
\hline UDC80 & 70 & SP1T & -- & 1.881 & 2.89 & 1.828 & 0.466 & 16.6 & 26.5 & 68.2 & 5.3 \\
\hline RDC81 & 6 & $\mathrm{HC}$ & 35 & 1.904 & $\dagger t$ & -- & -- & -- & -- & -- & -- \\
\hline
\end{tabular}

* Identifies the intended type of test; see text for explanation of symbols.

* Reverse strain path tests.

$\dagger$ Strain-rate tests.

t† Membrane leaked during test; no water content value available 
Table 2.2 Test notes.

\begin{tabular}{|c|c|c|c|}
\hline Test No. & $\begin{array}{l}\text { Plate } \\
\text { No. }\end{array}$ & $\begin{array}{l}\text { Type } \\
\text { of Test* } \\
\end{array}$ & Test Notes \\
\hline $\mathrm{RDC0} 1$ & 1 & $\mathrm{HC}$ & $\begin{array}{l}\text { Confining pressure cycled at } 17.2 \text { and } 34.7 \mathrm{MPa} \text {; } \\
\text { peak pressure was } 58.3 \mathrm{MPa} \text {. }\end{array}$ \\
\hline $\mathrm{RDCO} 2$ & 2 & $\mathrm{HC}$ & $\begin{array}{l}\text { Confining pressure cycled at } 17.2 \text { and } 34.5 \mathrm{MPa} \text {; } \\
\text { peak pressure was } 58.9 \mathrm{MPa} \text {. }\end{array}$ \\
\hline $\mathrm{RDCO} 3$ & 3 & $\mathrm{HC}$ & $\begin{array}{l}\text { Confining pressure cycled at } 17.1 \text { and } 34.8 \mathrm{MPa} \text {; } \\
\text { peak pressure was } 59.1 \mathrm{MPa} \text {. }\end{array}$ \\
\hline $\mathrm{RDCO} 4$ & 7 & CTC & $\begin{array}{l}\text { Specimen diameter was } 5.40 \mathrm{~cm} \text {; used standard piston } \\
\text { and piston load cell; good test. }\end{array}$ \\
\hline RDC05 & 8 & CTC & $\begin{array}{l}\text { Specimen diameter was } 7.63 \mathrm{~cm} \text {; used standard piston } \\
\text { and piston load cell; test conducted to evaluate } \\
\text { effects of specimen size; good test. }\end{array}$ \\
\hline $\mathrm{RDC0} 6$ & 10 & RTE & $\begin{array}{l}\text { Movement of extension loading piston was restricted } \\
\text { by top plug; realized that the base pedestal height } \\
\text { must be matched to the type of test and the } \\
\text { anticipated strains during HC loading. }\end{array}$ \\
\hline $\mathrm{RDC0} 7$ & 11 & RTE & $\begin{array}{l}\text { Shorter base pedestal used; movement of piston } \\
\text { again restricted by top plug. }\end{array}$ \\
\hline $\mathrm{RDC08}$ & -- & RTE & $\begin{array}{l}\text { Membrane leaked during either HC or TXE loading; no } \\
\text { data available. }\end{array}$ \\
\hline RDCO9 & 16 & CTC/RTE & $\begin{array}{l}\text { Good test; maximum } 0.17-\mathrm{MPa} \text { change in Iateral } \\
\text { stress during loading and unloading; specimen } \\
\text { separated upon failure; no posttest water content. }\end{array}$ \\
\hline RDC 10 & 17 & CTC/RTE & $\begin{array}{l}\text { Good test; specimen separated upon failure; no } \\
\text { posttest water content. }\end{array}$ \\
\hline $\mathrm{RDC} 11$ & 18 & $\mathrm{RTE} / \mathrm{CTC}$ & $\begin{array}{l}\text { Good test; unloading after shear compression phase } \\
\text { was unsuccessful due to restriction of extension } \\
\text { loading piston. }\end{array}$ \\
\hline $\mathrm{RDC} 12$ & 19 & $\mathrm{RTE} / \mathrm{CTC}$ & $\begin{array}{l}\text { Good test; specimen separated upon failure during } \\
\text { second shear extension phase; no posttest water } \\
\text { content. }\end{array}$ \\
\hline RDC 13 & 12 & CTE & $\begin{array}{l}\text { Membrane leaked during loading; constant axial } \\
\text { stress condition was not maintained. }\end{array}$ \\
\hline RDC 14 & 13 & CTE & $\begin{array}{l}\text { Unable to maintain constant axial stress } \\
\text { conditions; specimen separated upon failure; no } \\
\text { posttest water content. }\end{array}$ \\
\hline RDC 15 & 14 & CTE & $\begin{array}{l}\text { Good test; some variation in axial stress occurred } \\
\text { during test; specimen separated upon failure; no } \\
\text { posttest water content. }\end{array}$ \\
\hline RDC 16 & 15 & CTE & $\begin{array}{l}\text { Good test; some variation in axial stress occurred } \\
\text { during test; specimen separated upon failure; no } \\
\text { posttest water content. }\end{array}$ \\
\hline
\end{tabular}


Table 2.2. (Conti nued).

\begin{tabular}{|c|c|c|c|}
\hline Test No. & $\begin{array}{l}\text { Plate } \\
\text { No. }\end{array}$ & $\begin{array}{c}\text { Type } \\
\text { of Test* } \\
\end{array}$ & Test Notes \\
\hline RDC17 & 20 & $\mathrm{RTC} / \mathrm{CTE}$ & $\begin{array}{l}\text { Good test; maximum } 0.17-\mathrm{MPa} \text { change in vertical } \\
\text { stress during test; specimen separated upon } \\
\text { failure; no posttest water content. }\end{array}$ \\
\hline $\mathrm{RDC} 18$ & 21 & $\mathrm{RTC} / \mathrm{CTE}$ & $\begin{array}{l}\text { Good test; maximum } 0.17-\mathrm{MPa} \text { change in vertical } \\
\text { stress during test; specimen separated upon } \\
\text { failure; no posttest water content. }\end{array}$ \\
\hline RDC19 & 4 & $\mathrm{HC}$ & $\begin{array}{l}\text { Confining pressure cycled at } 17.0 \text { and } 34.6 \mathrm{MPa} \text {; } \\
\text { peak pressure was } 59.0 \mathrm{MPa} \text {. }\end{array}$ \\
\hline RDC20 & 32 & SP $3 A$ & $\begin{array}{l}\text { Piston was not adequately seated during the initial } \\
\text { SP loading; this resulted in extensive tilting of } \\
\text { the specimen top cap and produced a significant } \\
\text { movement of of the desired SP; increasing lateral } \\
\text { strains were observed during this initial loading. }\end{array}$ \\
\hline $\mathrm{RDC} 21$ & 33 & SP3A & $\begin{array}{l}\text { Some tilting of specimen top cap occurred during } \\
\text { the initial SP loading. }\end{array}$ \\
\hline RDC22 & 34 & SP3A & $\begin{array}{l}\text { Again, some tilting of specimen top cap occurred } \\
\text { during the initial SP loading; increasing lateral } \\
\text { strains were again observed during this initial } \\
\text { loading. }\end{array}$ \\
\hline RDC23 & 9 & CTC & $\begin{array}{l}\text { Test conducted to compare the resulting strains } \\
\text { with SP3A; increasing lateral strains were again } \\
\text { observed during initial loading. }\end{array}$ \\
\hline $\mathrm{RDC} 24$ & 51 & SP2B & $\begin{array}{l}\text { Good test; did not stay on the SP at the end of the } \\
\text { test. }\end{array}$ \\
\hline $\mathrm{RDC} 25$ & 52 & SP 2B & $\begin{array}{l}\text { Problem developed with vertical deformeter; test as } \\
\text { terminated prior to completion of SP; data } \\
\text { questionable. }\end{array}$ \\
\hline $\mathrm{RDC} 26$ & 53 & SP2B & $\begin{array}{l}\text { Good test; returned to a zero stress difference } \\
\text { state before reaching the point of lateral strain } \\
\text { reversal. }\end{array}$ \\
\hline $\mathrm{RDC} 27$ & 54 & SP2B & $\begin{array}{l}\text { Good test; again, did not reach the point of } \\
\text { lateral strain reversal. }\end{array}$ \\
\hline RDC28 & 35 & SP $3 B$ & $\begin{array}{l}\text { Good test; increasing lateral strains during } \\
\text { initial SP loading caused some problems; observed a } \\
\text { distinct period of constant stress difference with } \\
\text { increasing vertical strain. }\end{array}$ \\
\hline RDC29 & 36 & SP $3 B$ & $\begin{array}{l}\text { Good test; again, observed a distinct period of } \\
\text { constant stress difference with increasing vertical } \\
\text { strain. }\end{array}$ \\
\hline RDC30 & 37 & SP 3B & $\begin{array}{l}\text { Good test; again, observed a distinct period of } \\
\text { constant stress difference with increasing vertical } \\
\text { strain. }\end{array}$ \\
\hline
\end{tabular}


Table 2.2. (Continued).

\begin{tabular}{|c|c|c|c|}
\hline Test No. & $\begin{array}{l}\text { Plate } \\
\text { No. }\end{array}$ & $\begin{array}{c}\text { Type } \\
\text { of Test* } \\
\end{array}$ & Test Notes \\
\hline RDC31 & 39 & SP 3C & $\begin{array}{l}\text { Good test; period of constant stress difference was } \\
\text { observed during this test; similar to SP3B. }\end{array}$ \\
\hline RDC32 & 40 & SP3C & $\begin{array}{l}\text { Good test; again, observed a distinct period of } \\
\text { constant stress difference with increasing vertical } \\
\text { strain. }\end{array}$ \\
\hline RDC33 & 41 & SP 3C & $\begin{array}{l}\text { Good test; again, observed a distinct period of } \\
\text { constant stress difference with increasing vertical } \\
\text { strain. }\end{array}$ \\
\hline RDC 34 & 5 & $\mathrm{HC}$ & $\begin{array}{l}\text { Confining pressure cycled at } 13.7 \text { and } 34.4 \mathrm{MPa} \text {; } \\
\text { peak pressure was } 58.7 \mathrm{MPa} \text {. }\end{array}$ \\
\hline RDC35 & 48 & $\mathrm{SP} 2 \mathrm{~A}$ & $\begin{array}{l}\text { Large increase in lateral strain occurred during } \\
\text { the initial SP loading; tilting of top cap was part } \\
\text { of the problem. }\end{array}$ \\
\hline RDC36 & 49 & SP2A & $\begin{array}{l}\text { Increase in lateral strain occurred during the } \\
\text { initial SP loading; specimen was able to reach the } \\
\text { point of lateral strain reversal. }\end{array}$ \\
\hline RDC37 & 50 & SP2A & $\begin{array}{l}\text { Good test; again, the specimen was able to reach } \\
\text { the point of lateral strain reversal. }\end{array}$ \\
\hline RDC38 & 55 & SP2C & $\begin{array}{l}\text { Large increase in lateral strain occurred during } \\
\text { the initial SP loading; tilting of top cap was } \\
\text { still a significant part of the problem. }\end{array}$ \\
\hline RDC 39 & 22 & CTC/RTE & $\begin{array}{l}\text { Good test; maximum } 0.28-\mathrm{MPa} \text { change in lateral } \\
\text { stress during the entire test; axial strains during } \\
\text { the extension cycles were approximately }-5,-4 \text {, \& } \\
-5 \% \text { respectively; specimen separated upon failure; } \\
\text { no posttest water content. }\end{array}$ \\
\hline $\mathrm{RDC} 40$ & 23 & CTC/RTE & $\begin{array}{l}\text { Good test; attempted to repeat test RDC } 39 \text {; problems } \\
\text { developed during the final unloading; movement of } \\
\text { the extension loading piston was restricted. }\end{array}$ \\
\hline RDC41 & -- & CTC/RTE & $\begin{array}{l}\text { Broken wire on internal film pot; no valuable data } \\
\text { obtained. }\end{array}$ \\
\hline RDC42 & 24 & CTC/RTE & $\begin{array}{l}\text { Good test; maximum } 0.28-\mathrm{MPa} \text { change in lateral } \\
\text { stress during the entire test; axial strains during } \\
\text { the extension cycles were approximately }-2 \% \text {; } \\
\text { specimen separated upon failure; no posttest water } \\
\text { content. }\end{array}$ \\
\hline $\mathrm{RDC} 43$ & 25 & $\mathrm{CTC} / \mathrm{RTE}$ & $\begin{array}{l}\text { Good test; maximum } 0.28-\mathrm{MPa} \text { change in lateral } \\
\text { stress during the entire test; axial strains during } \\
\text { the extension cycles were approximately }-4 \% \text {; final } \\
\text { unloading data were deleted due to restricted } \\
\text { movement of the extension loading piston; specimen } \\
\text { separated upon failure; no posttest water content. }\end{array}$ \\
\hline
\end{tabular}


Table 2.2. (Conti nued).

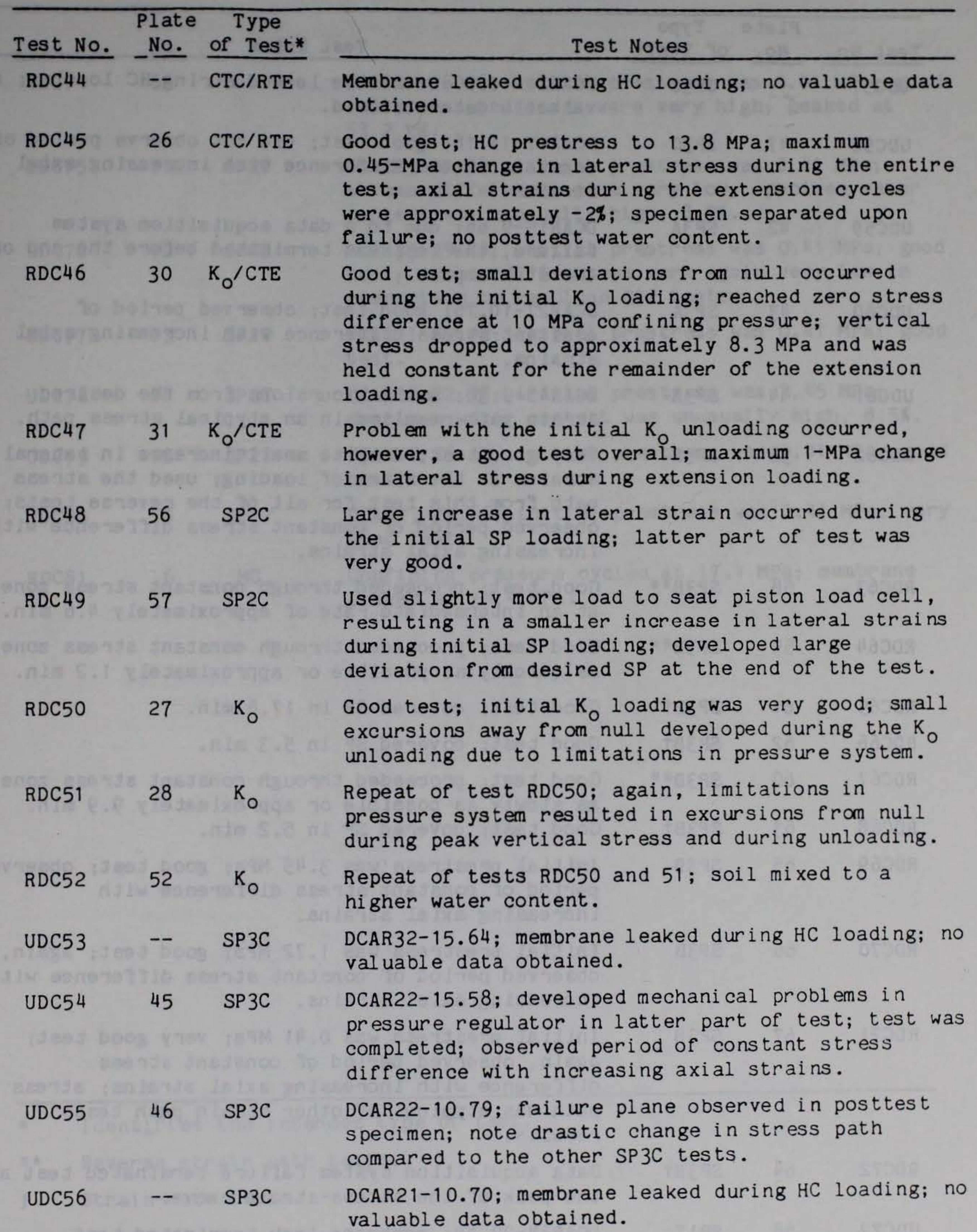
valuable data obtained. 
Table 2.2. (Conti nued).

\begin{tabular}{|c|c|c|c|}
\hline Test No. & $\begin{array}{l}\text { Plate } \\
\text { No. }\end{array}$ & $\begin{array}{c}\text { Type } \\
\text { of Test* } \\
\end{array}$ & Test Notes \\
\hline UDC57 & -- & SP 3C & $\begin{array}{l}\text { DCAR21-10.70; membrane leaked during HC loading; no } \\
\text { valuable data obtained. }\end{array}$ \\
\hline UDC58 & 47 & SP3C & $\begin{array}{l}\text { DCAR17-11.16; good test; did not observe period of } \\
\text { constant stress difference with increasing axial } \\
\text { strains. }\end{array}$ \\
\hline UDC59 & 42 & SP $3 A$ & $\begin{array}{l}\text { DCAR } 15-9.66 \text {; due to a data acquisition system } \\
\text { failure, the test was terminated before the end of } \\
\text { the strain path. }\end{array}$ \\
\hline UDC60 & 43 & SP3A & $\begin{array}{l}\text { DCAR27-10.76; good test; observed period of } \\
\text { constant stress difference with increasing axial } \\
\text { strains. }\end{array}$ \\
\hline UDC61 & 44 & SP $3 A$ & $\begin{array}{l}\text { DCAR } 45-9.30 \text {; small excursions from the desired } \\
\text { strain path resulted in an atypical stress path. }\end{array}$ \\
\hline RDC62 & 38 & SP $3 B$ & $\begin{array}{l}\text { Very good test, despite small increase in lateral } \\
\text { strains at the start of loading; used the stress } \\
\text { path from this test for all of the reverse tests; } \\
\text { observed period of constant stress difference with } \\
\text { increasing axial strains. }\end{array}$ \\
\hline RDC63 & 58 & $\mathrm{SP} 3 \mathrm{~B}^{* *}$ & $\begin{array}{l}\text { Good test; proceeded through constant stress zone } \\
\text { at an intermediate rate of approximately } 4.6 \mathrm{~min} \text {. }\end{array}$ \\
\hline $\mathrm{RDC} 64$ & 59 & SP3B** & $\begin{array}{l}\text { Good test; proceeded through constant stress zone } \\
\text { as quickly as possible or approximately } 1.2 \mathrm{~min} \text {. }\end{array}$ \\
\hline RDC65 & 61 & SP3Bt & Good test; covered SP in $17.6 \mathrm{~min}$. \\
\hline RDC66 & 62 & SP $3 B+$ & Good test; covered SP in $5.3 \mathrm{~min}$. \\
\hline RDC67 & 60 & $\mathrm{SP} 3 \mathrm{~B}^{* *}$ & $\begin{array}{l}\text { Good test; proceeded through constant stress zone } \\
\text { as slowly as possible or approximately } 9.9 \mathrm{~min} \text {. }\end{array}$ \\
\hline $\mathrm{RDC6} 8$ & 63 & SP3Bt & Good test; covered SP in $5.2 \mathrm{~min}$. \\
\hline RDC69 & 65 & SP $3 B$ & $\begin{array}{l}\text { Initial prestress was } 3.45 \mathrm{MPa} \text {; good test; observed } \\
\text { period of constant stress difference with } \\
\text { increasing axial strains. }\end{array}$ \\
\hline RDC70 & 66 & SP3B & $\begin{array}{l}\text { Initial prestress was } 1.72 \mathrm{MPa} \text {; good test; again, } \\
\text { observed period of constant stress difference with } \\
\text { increasing axial strains. }\end{array}$ \\
\hline RDC71 & 67 & SP $3 B$ & $\begin{array}{l}\text { Initial prestress was } 0.41 \mathrm{MPa} \text {; very good test; } \\
\text { again, observed period of constant stress } \\
\text { difference with increasing axial strains; stress } \\
\text { path was atypical of other strain path tests } \\
\text { results. }\end{array}$ \\
\hline RDC72 & 64 & SP $3 B t$ & $\begin{array}{l}\text { Data acquisition system failure terminated test at } \\
\text { the peak of the stress-strain curve. }\end{array}$ \\
\hline UDC73 & 68 & SP1T & DCAR38-29.44; membrane leak terminated test. \\
\hline
\end{tabular}


Table 2.2. (Continued).

\begin{tabular}{|c|c|c|c|}
\hline Test No. & $\begin{array}{l}\text { Plate } \\
\text { No. }\end{array}$ & $\begin{array}{c}\text { Type } \\
\text { of Test* }\end{array}$ & Test Notes \\
\hline UDC7 4 & 69 & SP1T & $\begin{array}{l}\text { DCAR21-26.06; initial prestress was } 0.41 \mathrm{MPa} \text {; good } \\
\text { test; measured loads were very high; peaked at } \\
53.2 \mathrm{kN} \text {. }\end{array}$ \\
\hline UDC75 & 71 & SP2T & $\begin{array}{l}\text { DCAR23-22.98; initial prestress was } 0.41 \mathrm{MPa} \text {; } \\
\text { stress differences were very low; specimen water } \\
\text { content was unusually high, } 8.9 \% \text {. }\end{array}$ \\
\hline UDC76 & 72 & SP2T & $\begin{array}{l}\text { DCAR33-29.29; initial prestress was } 0.41 \mathrm{MPa} \text { good } \\
\text { test; stress differences were again very low in } \\
\text { comparison to remolded SP2 tests. }\end{array}$ \\
\hline UDC77 & 73 & SP2T & $\begin{array}{l}\text { DCAR21-23.07; initial prestress was } 0.41 \mathrm{MPa} \text {; good } \\
\text { test. }\end{array}$ \\
\hline UDC7 8 & 74 & SP2T & $\begin{array}{l}\text { DCAR23-22.98; initial prestress was } 3.45 \mathrm{MPa} \text {; } \\
\text { specimen water content was unusually high, } 8.5 \% \text {. }\end{array}$ \\
\hline UDC79 & 75 & SP2T & $\begin{array}{l}\text { DCAR 42-29.32; initial prestress was } 0.41 \mathrm{MPa} \text {; good } \\
\text { test. }\end{array}$ \\
\hline UDC80 & 70 & SP1T & $\begin{array}{l}\text { DCAR23-24.66; initial prestress was } 0.41 \mathrm{MPa} \text {; very } \\
\text { good test. }\end{array}$ \\
\hline RDC81 & 6 & $\mathrm{HC}$ & $\begin{array}{l}\text { Confining pressure cycled at } 17.1 \mathrm{MPa} \text {; membrane } \\
\text { leaked at } 34.4 \mathrm{MPa} \text {. }\end{array}$ \\
\hline
\end{tabular}

* Identifies the intended type of test.

** Reverse strain path tests.

$\dagger \quad$ Strain-rate tests. 


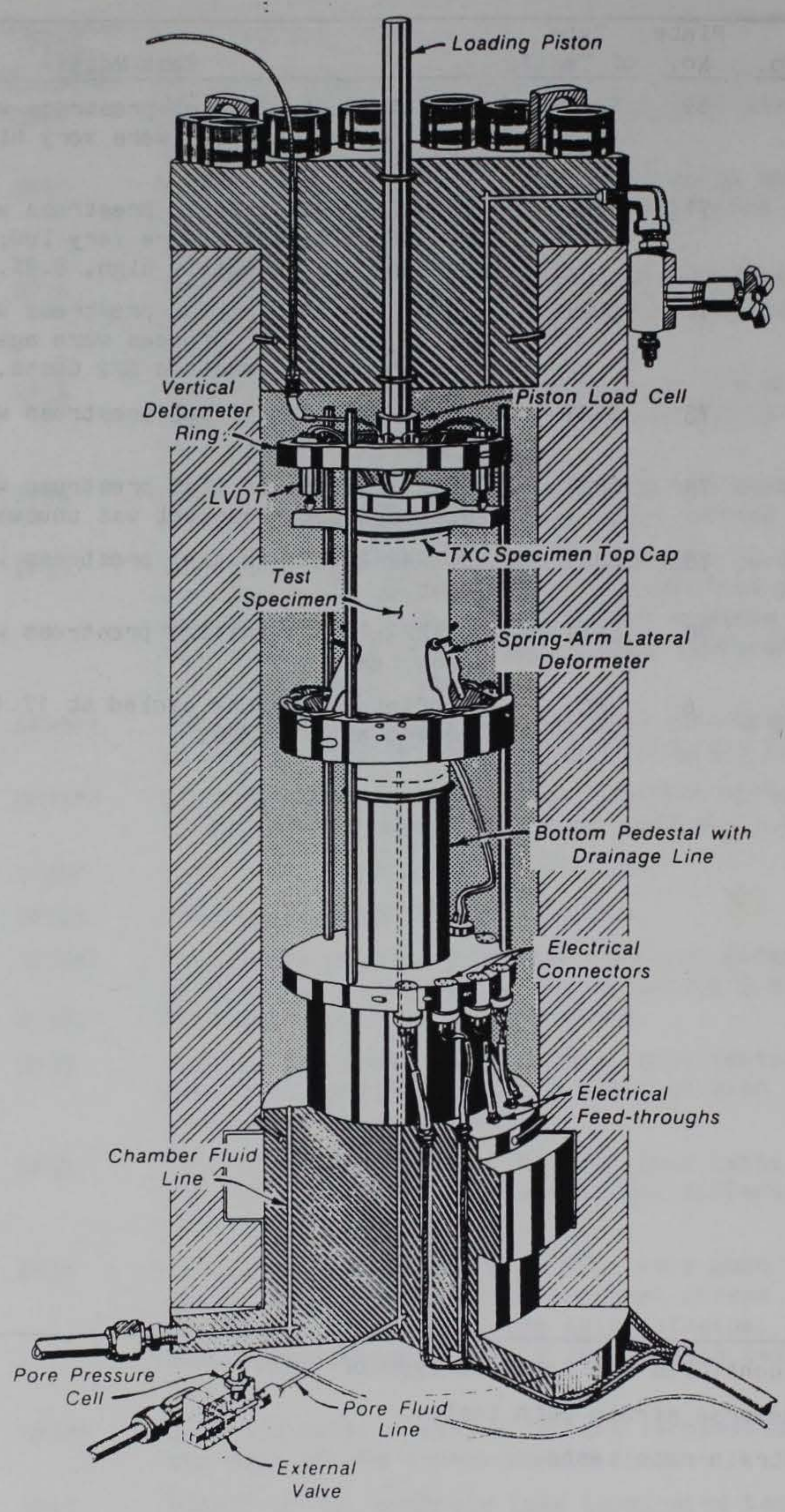

Figure 2.1. Schematic of test device with TXC top cap. 


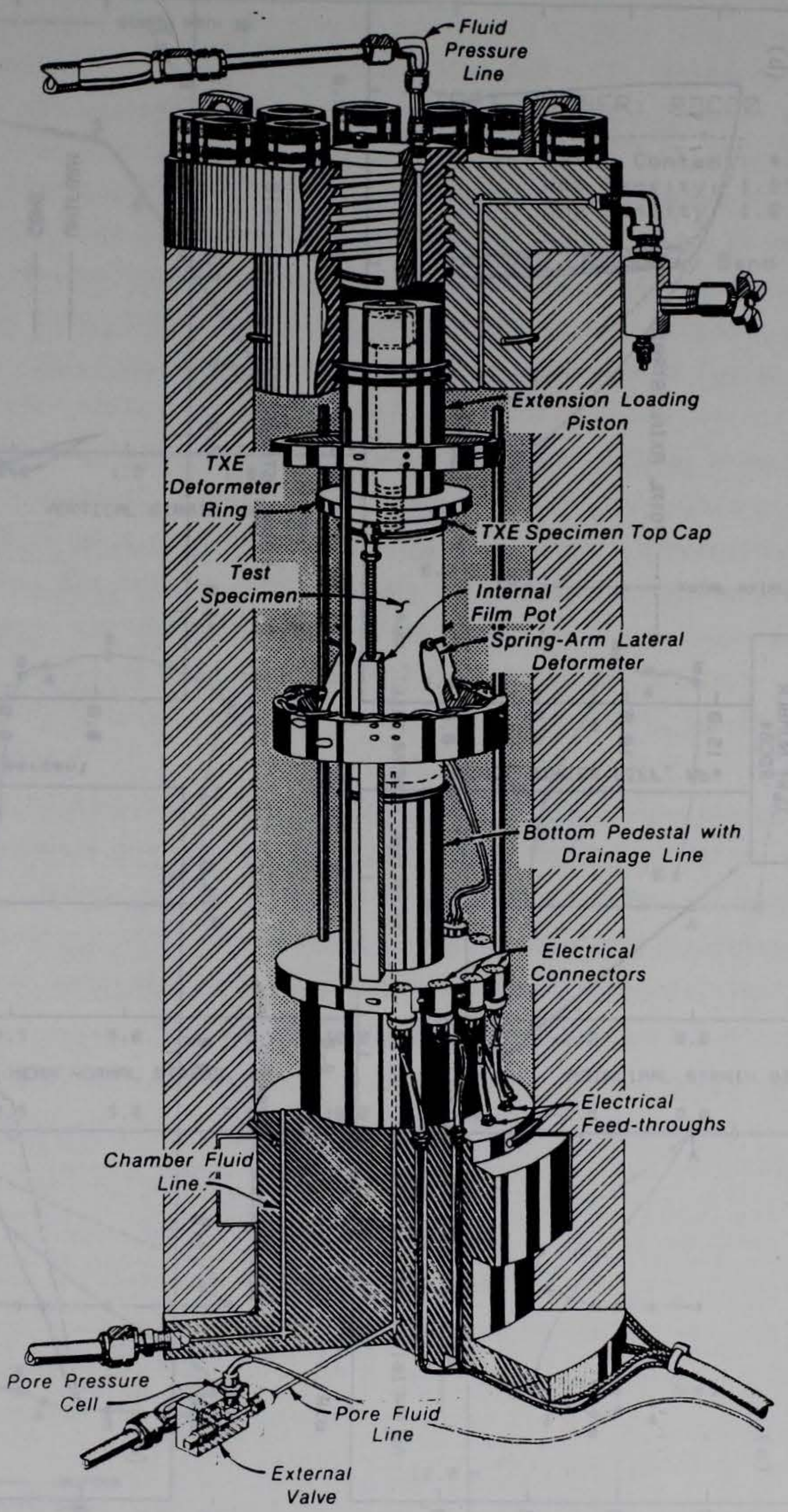

Figure 2.2 Schematic of test device with TXE top cap. 


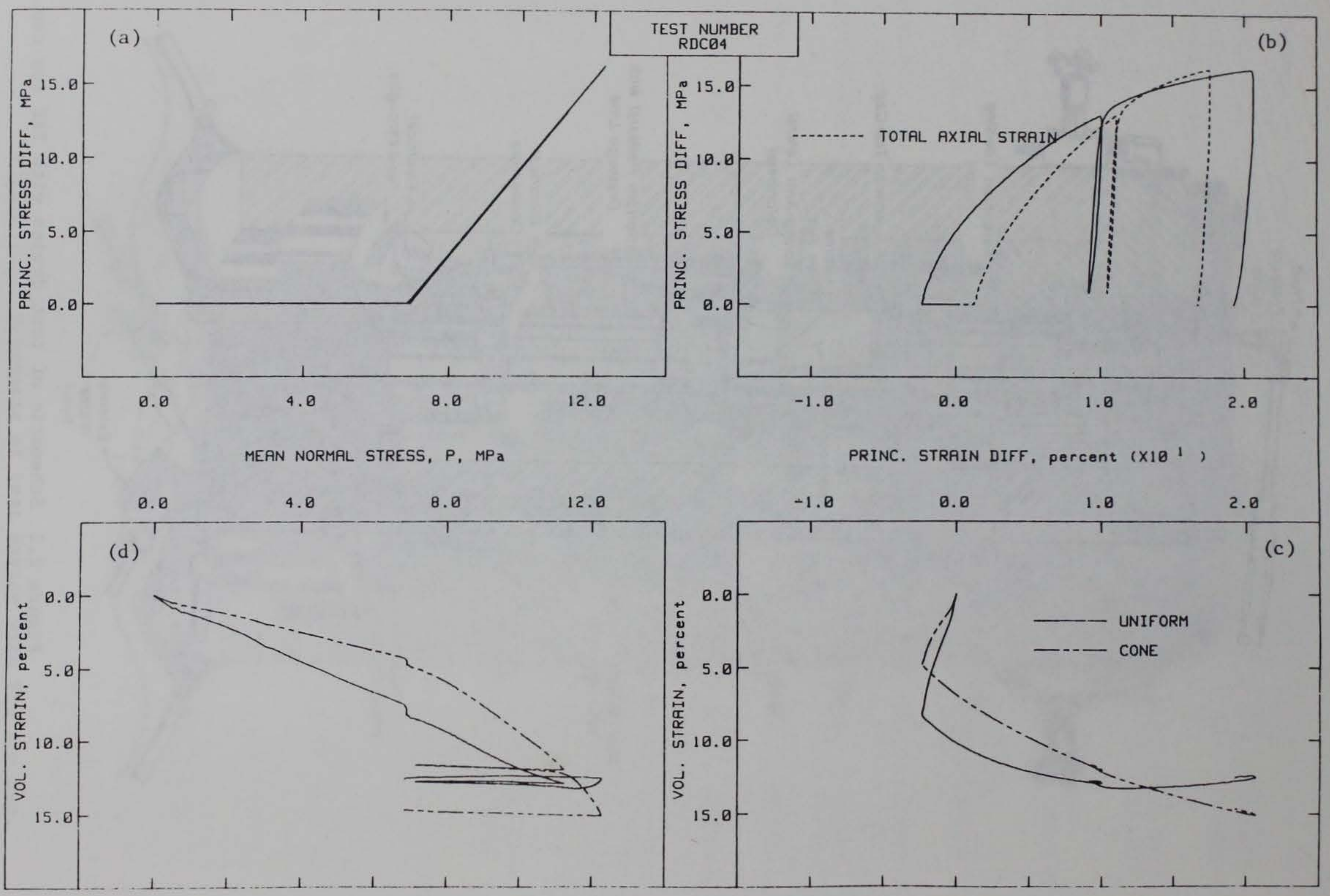

Figure 2.3. Example of four-corner plot. 


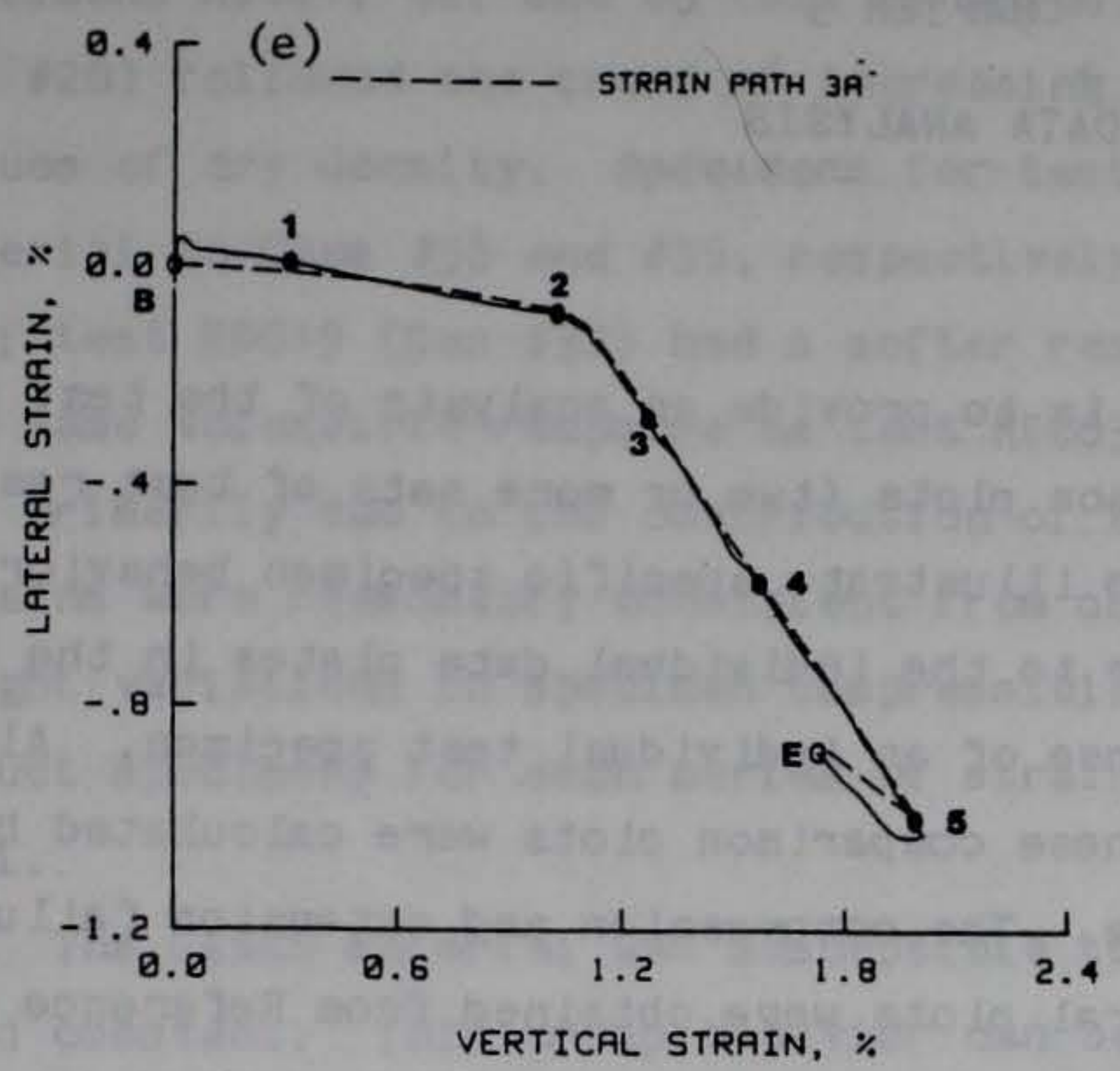

TEST NUMBER: RDCZØ

Water Content: $4.81 \%$

Wet Density: $1.899 \mathrm{~g} / \mathrm{cc}$

Dry Density: $1.812 \mathrm{~g} / \mathrm{cc}$

Remolded CRRES-Dry Sand
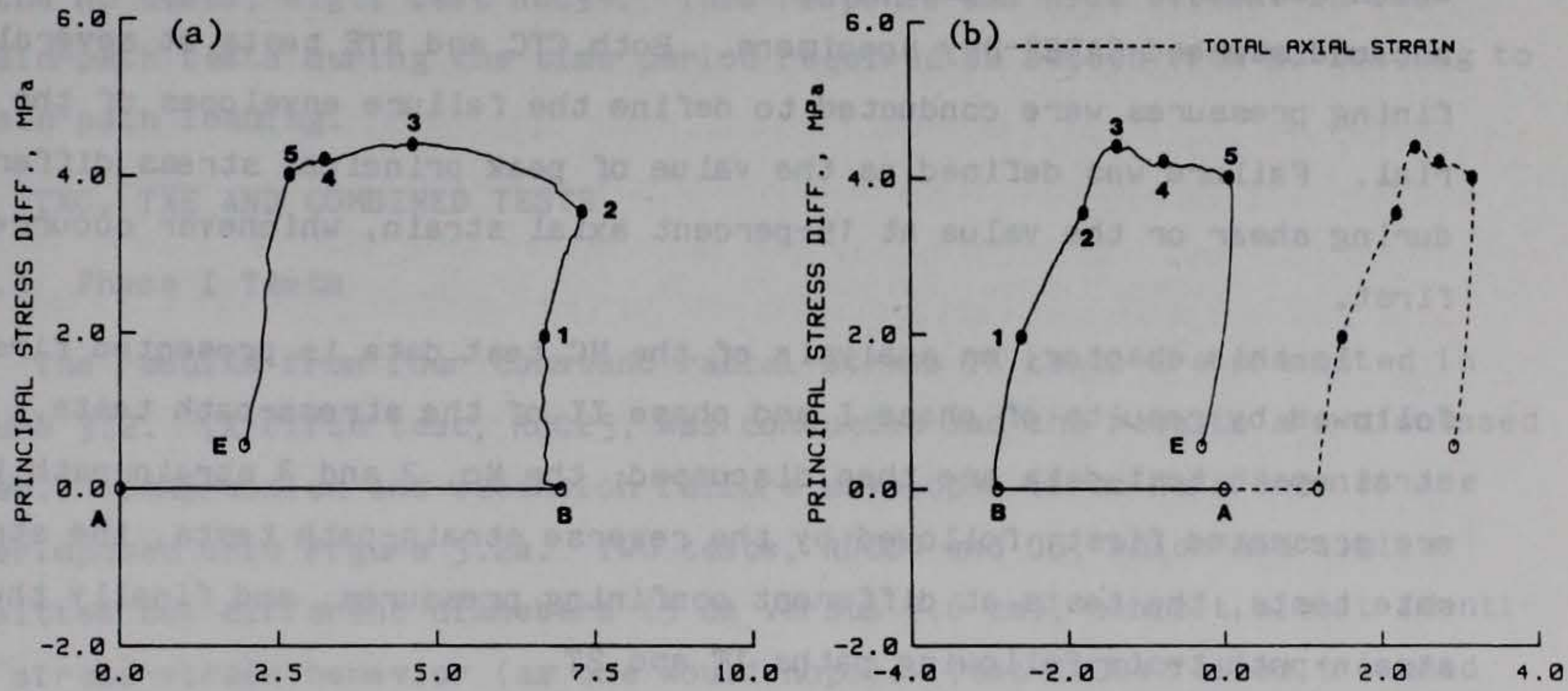

MEAN NORMAL STRESS, MPa
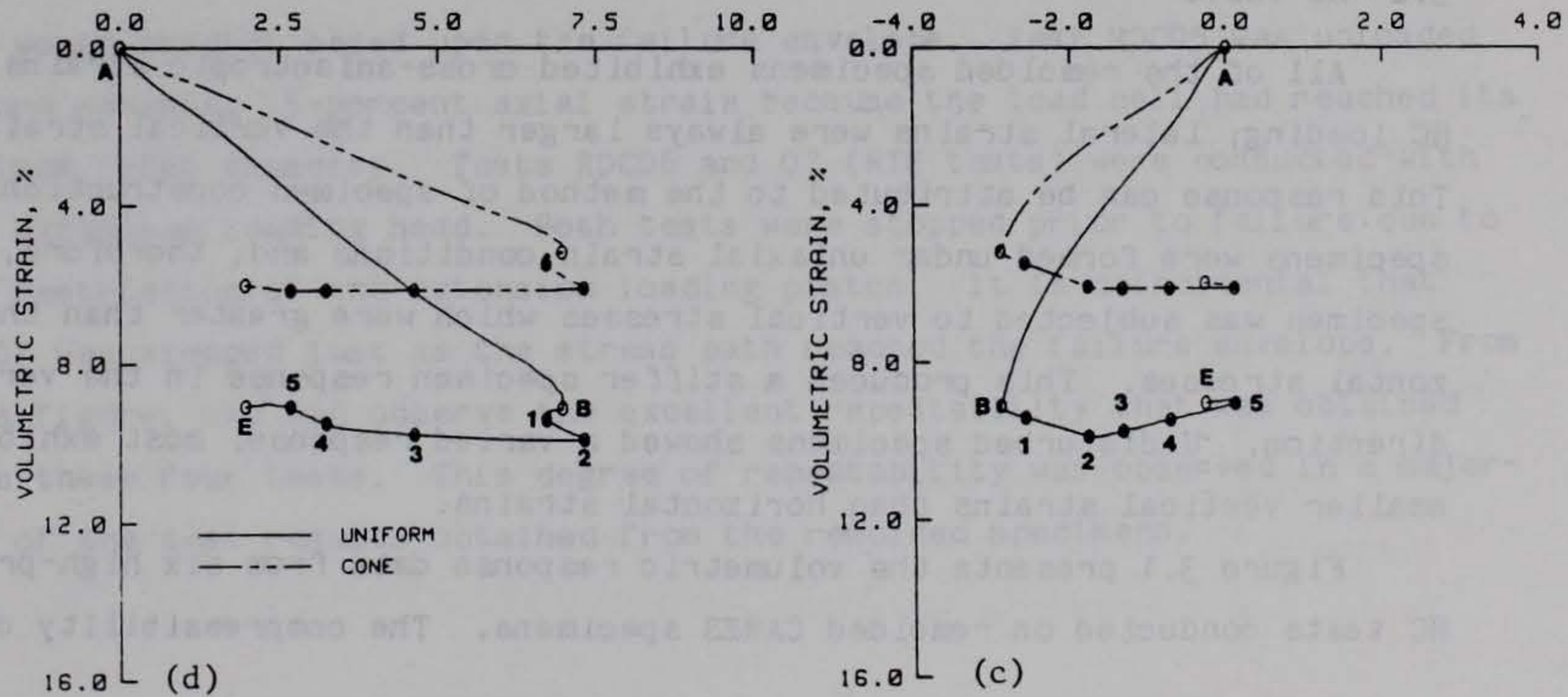

Figure 2.4. Example of five-plot figure. 
CHAPTER 3

DATA ANALYSIS

\subsection{FORMAT OF DATA PRESENTATION}

The purpose of this chapter is to provide an analysis of the test data presented in Chapter 2. Comparison plots (two or more sets of test results per plot) are used extensively to illustrate specific specimen behavior or group behavior. One should refer to the individual data plates in the back of this report to discern the response of an individual test specimen. Also note that the volumetric strains in these comparison plots were calculated by assuming a uniform specimen shape. The compression and extension $f$ ailure envelopes superimposed upon several plots were obtained from Reference 8 , which documents the results of a laboratory test program conducted on remolded and undisturbed CARES-Dry specimens. Both CTC and RTE tests at several confining pressures were conducted to define the failure envelopes of the material. Failure was defined as the value of peak principal stress difference during shear or the value at 15-percent axial strain, whichever occurred first.

In this chapter, an analysis of the HC test data is presented $\mathrm{f}$ irst, followed by results of phase I and phase II of the stress-path tests. The strain-path test data are then discussed; the No. 2 and 3 strain-path tests are presented first, followed by the reverse strain-path tests, the strainrate tests, the tests at different confining pressures, and finally the strain-path tests following paths $1 \mathrm{~T}$ and $2 \mathrm{~T}$.

\subsection{HC TESTS}

All of the remolded specimens exhibited cross-anisotropic strains during HC loading; lateral strains were always larger than the vertical strains. This response can be attributed to the method of specimen construction. The specimens were formed under uniaxial strain conditions and, therefore, each specimen was subjected to vertical stresses which were greater than the horizontal stresses. This produced a stiffer specimen response in the vertical direction. Undisturbed specimens showed a varied response; most exhibited smaller vertical strains than horizontal strains.

Figure 3.1 presents the volumetric response data from six high-pressure HC tests conducted on remolded CARES specimens. The compressibility of 
specimens RDC01, 02, and 03 (all of which were prepared from materials in Can \#20) followed the trend of increasing volumetric strains with decreasing values of dry density. Specimens for tests RDC34 and RDC81 (prepared from material in Cans $\$ 36$ and $\$ 35$, respectively) had identical volumetric responses; test RDC19 (Can \#32) had a softer response than RDC34 and approximately the same volumetric response as test RDC03. The variability in the HC data was primarily due to the contribution of the radial strains because the axial strains were reasonably consistent from one test to another. Because of these slight variations in specimen compressibility, an attempt was made to construct specimens for each series of strain-path tests from one can of material.

The CARES material was susceptible to creep when confining pressures were held constant. This creep behavior can be observed at peak stress in several of the HC tests, e.8., test RDC34. This response was also evident in the strain-path tests during the time period required to switch from HC loading to strain-path loading.

\subsection{TXC, TXE AND COMBINED TESTS}

\subsection{Phase I Tests}

The results from four constant radial stress $T X$ tests are presented in Figure 3.2. (A fifth test, RDC23, was conducted and the results are discussed later.) Compression and extension failure envelopes (from Reference 8) are superimposed onto Figure 3.2a. Two tests, RDCO4 and 05, which had similar densities but different diameters ( $5 \mathrm{~cm}$ versus $7.6 \mathrm{~cm}$ ), exhibit almost identical stress-strain behavior (as one would hope). Test RDC04 failed (reached 15-percent axial strain) at a slightly lower value of stress difference than one would predict based upon the failure envelope. Test RDC05 was unloaded before reaching 15-percent axial strain because the load cell had reached its maximum rated capacity. Tests RDC06 and 07 (RTE tests) were conducted with the extension loading head. Both tests were stopped prior to failure due to the restriction of the extension loading piston. It is coincidental that RDC07 was stopped $j$ ust as the stress path reached the failure envelope. From this figure, one can observe the excellent repeatability that was obtained from these four tests. This degree of repeatability was observed in a majority of the test results obtained from the remolded specimens. 
Stress and strain data from four CTE tests are presented in Figure 3.3 . Note the two different stress paths that were followed in these four tests. Tests RDC13 and 14 were not conducted under "true" constant axial stress conditions. In reality, the axial stress decreased during shear loading, thereby producing the steeper stress paths. After correcting the source of the problem, tests RDC15 and 16 were conducted such that axial stresses were held much closer to a constant value during shear. During test RDC13, the membrane leaked before failure was achieved. Tests RDC14 and 15 failed as the stress path reached the extension failure envelope. Test RDC16 failed as its stress path reached a point just above but in close proximity to the extension failure envelope.

Results from the first two combined stress-path tests, RDC09 and 10, are presented in Figure 3.4. These tests were designed to push the failure envelope out as far as possible by deforming the specimen to 15-percent axial strain in compression, then unloading through zero stress difference and into extension until the specimen failed. Both specimens failed in extension by separating at approximately their midheight, which in turn, caused the membrane to leak. In Figure 3.5, one can observe that the two stress paths just miss the compression failure envelope during initial loading and that each specimen failed as its stress path reached the extension failure envelope.

In Figure 3.6, the results of three CTC tests, RDC04, 05, and 23, are presented with the results from test RDCO9 (CTC/RTE) in order to compare the compression data obtained from the two loading systems, i.e., the conventional TXC and the TXE loading system. The stress-strain data from the three CTC tests are in excellent agreement. However, test RDCO9 (test RDC10 had a similar response) had a slightly stiffer stress-strain curve and reached higher values of stress difference than test RDC04. This response was caused by the stress path of test RDCO9 falling to the right of a $3: 1$ slope, meaning that "true" constant radial stress conditions were not maintained during the entire loading phase of the test. This observation brings to light a very important point, i.e., when comparing stress-strain curves from different types of tests, one must make sure that the same stress path was followed in all of the tests.

The results from two RTE/CTC tests, RDC11 and 12, are presented in Figure 3.7; the two stress paths are plotted to a larger scale in Figure 3.8. Again, one can observe the excellent repeatability of the test data. The 
stress paths of both specimens fall just short of the failure envelope in extension, then hit the failure envelope in compression. Specimen RDC12, after being stressed to the compression failure envelope, failed during its final unloading into extension when its stress path reached a point significantly above the extension failure envelope. This observation is addressed again at the conclusion of the next section.

Results from two RTC/CTE tests, RDC17 and 18, are presented in Figure 3.9. The stress paths of both specimens reach and stay on the compression failure envelope while both specimens undergo significant amounts of axial strain. During the time the stress paths were in contact with the failure envelope, both specimens were dilating significantly (based on the uniform shape assumption for computing volumetric strains). Then, in extension, they dramatically fail before reaching the extension $f$ ailure envelope (Figure 3.9a). Note that CTE tests RDC14, 15 and 16 (Figure 3.3), which did not undergo the compression loading, failed when their stress paths reached the extension failure envelope.

\subsubsection{Phase II Tests}

Five CTC/RTE tests, RDC39, 40, 42, 43, and 45, were successfully conducted under constant radial stress boundary conditions, and provided a measure of the response of the CARES material under different load-unloadreload conditions. In these tests, the constitutive modelers were particularly interested in the slopes of the reloading stress-strain curves and the position of the reloading curve relative to the point of initial unloading. As described in Section 2.6.2, the specimens were deformed to specific axial strains in compression and in extension during each test. One of the five tests (RDC45) was prestressed to $13.8 \mathrm{MPa}$, as opposed to the 6.9-MPa prestress for the other four tests.

The stress-strain curves of the four tests prestressed to $6.9 \mathrm{MPa}$ are presented in Figure 3.10. These curves are identical through the first loadunload cycle for all four specimens. After loading in extension to different values of axial strain, the stress-strain curves then diverge upon reloading. One can now observe a most interesting response, i.e., the specimens deformed to the larger values of strain in extension reloaded in compression to higher values of stress difference. For example, test RDC42 (Figures 3.10 and 3.11) was deformed to -2-percent axial strain from the point of unloading and 
achieved a peak stress difference of approximately $16 \mathrm{MPa}$ before the second unloading cycle began. Test RDC $39^{\circ}$ was deformed to -5 -percent axial strain in extension and achieved a peak stress difference of approximately $18.5 \mathrm{MPa}$. This same trend is also evident during the first reloading cycle in tests RDC40 and 43 (Figure 3.10).

All of the CTC/RTE tests, RDC39, 40, 42, 43, and 45, were loaded in extension during one or more of the unloading cycles such that their stress paths reached or just missed the extension failure envelope. At the end of the tests, i.e., after the last loading cycle, the stress paths for all five tests extended to points significantly above the compression failure envelope. This response is illustrated in Figures 3.11 and 3.12 for tests RDC 39 and 42 , and in Figures 3.13 and 3.14 for tests RDC42 and 45 . This response could be an artifact of our definition of failure, i.e., 15-percent axial strain was used to determine the points on the failure envelope, whereas, in that last cycle of loading, the specimens were deformed to axial strains in excess of 15 percent. However, the response could also be attributed to an isotropic expansion of the failure surfaces caused by the axial deformations in extension. Recall that test RDCO4 (Figure 3.6), which was cycled to approximately zero stress difference, did not reach the failure envelope.

The phase I shear data can now be evaluated with respect to hardening response. With the exception of the RTC/CTE and RTE/CTC tests, all of the tests failed when their stress paths reached the failure envelope. The single RTE/CTC test (RDC12) appeared to fail prematurely, based upon an analysis of stress ratio values (vertical stress divided by lateral stress). The values of stress ratio from the RTC/CTE tests (RDC17 and 18) reached a minimum before specimen failure, therefore more confidence is placed on these data. Remember that the stress paths of the RTC/CTE tests moved up to the failure envelope, underwent a considerable amount of axial strain, then, during unloading into extension, failed above the failure envelope. The phase II tests showed some type of "stiffening" response. The specimens that were deformed to the larger values of strain in extension reloaded in compression to higher values of stress difference. This behavior was not observed when the specimen was unloaded to zero stress difference. In those cases, the stress-strain curve bends over upon reaching the point of initial unloading; there was no jump to higher values of stress difference. Although the existing data base is 
interesting, it is insufficient to characterize the hardening response of the CARES material as either isotropi $\ddot{c}$ or kinematic.

\section{$3.4 \mathrm{UX} / \mathrm{K}_{0}$ AND $\mathrm{K}_{\mathrm{O}} / \mathrm{CTE}$ TESTS}

The data from three $U X / K_{0}$ tests (RDC50, 51 and 52) are summarized in Figure 3.15. The data clearly indicate a change in response as a result of the increased water content of the specimen for test RDC52 ( 6.11 percent) versus the water contents of 3.81 and 3.89 percent for the specimens in tests RDC50 and 51, respectively. The dry density of specimen RDC52 was $1.78 \mathrm{~g} / \mathrm{cc}$ versus 1.81 and $1.82 \mathrm{~g} / \mathrm{CC}$ for specimens RDC50 and 51 . The stress paths and stress-strain curves of tests RDC50 and 51 are in good agreement up to approximately 10-MPa stress difference. All of the stress paths exhibit irregularities near peak stress; this was caused by the coarse pressure control of the hydraulic system during the later stages of loading and the early stages of unloading.

Two $\mathrm{K}_{\mathrm{o}}$ /CTE tests, RDC46 and 47, were also conducted in this test program. A $U X / K_{0}$ load and unload cycle was first imposed upon each specimen followed by CTE loading until failure. The results from these two tests are presented in Figure 3.16. The stress-path loading and unloading curves (Figure 3.16a) exhibit some variability due to the pumping action of the high-pressure supply and the lack of pressure control during pressure release. Despite the scatter in the stress paths, the other test results are very repeatable. The failure points are the most important response illustrated in Figure 3.16 in that the specimens failed in extension before reaching the failure envelope.

\subsection{TESTS FOLLOWING STRAIN PATHS 2 AND 3}

Two strain-path shapes (2 and 3 ), each having three levels of strain magnitude (A, B, and C) for a total of six strain paths, were investigated in this test program. Undisturbed specimens were tested only along strain paths $3 \mathrm{~A}$ and $3 \mathrm{C}$. To provide some indication of test control and specimen repeatability, at least three replications of each individual strain path were conducted. In the following two subsections, an analysis of the strain-path data is presented, beginning with SP3.

\subsubsection{SP3 Tests}

In each of the SP3A tests on remolded specimens (RDC20, 21, and 22), the lateral strains increased at the start of strain-path loading. At the same 
time, the top cap appeared to undergo some rearrangement or tilting, i.e., one vertical deformeter measured increasing deformations while the other indicated decreasing deformations. This problem appeared throughout the strain-path phase of the test program. At this point in the tests, the measured strain paths moved up off of the intended path requiring a reduction in confining pressure to bring the strain paths down to the intended path. These increasing lateral strains at the start of loading caused some concern in that one would normally expect negative lateral strains initially. In order to investigate this response further, a TXC test (RDC23) was conducted and careful attention was placed on measuring the strains at the start of loading. (A constant radial stress TXC test was conducted so as to eliminate the effect of changing confining pressure on the measured strains.) As depicted in Figure 3.17, the lateral strains of test RDC23 were initially positive up to point 1 , when they suddenly stopped changing while the vertical strains continued to increase past point 1. This behavior appears to be an artifact of the measurement system and is attributed to a shift or "seating" in the LVDT lateral deformeter probes at the early stages of loading (up to point 1). After the shift has occurred, the measurements are more realistic. This phenomenon becomes more pronounced as the strain magnitudes for each strain path decrease.

Figure 3.18 presents a comparison plot of the results from three SP $3 \mathrm{~A}$ tests, RDC20, 21, and 22. Although test RDC20 has a slightly larger value of negative principal strain difference at the end of $H C$, excellent repeatability was obtained in the three tests. Each of the three tests exhibited a "period of constant stress difference" (PCSD) during which axial strains increased while the stress paths were in the proximity of the failure envelope (Figure 3.18a). This response is characteristic of the No. 3 strain paths; it was also observed in the FY 83 test results on Nellis Baseline sand (Reference 1). This PCSD is discussed further in later sections of this report. The responses of the SP3C tests (RDC31, 32, and 33, Figure 3.19) were similar to those of the SP3A tests, except that the PCSD was more distinct in the SP3C data. If one has confidence in the validity of the uniform volumetric strain calculation, then the specimens were dilating during the PCSD; the truncated cone calculations indicate constant volumetric strain during the same period (see data plates). One should again note the excellent repeatability in the test data. 
Test results from the four SP3B tests, RDC28, 29, 30, and 62 (Figure 3.20), exhibit nearly identical responses. Again, the PCSD was very distinct in all of these tests. Test RDC62 had a slightly softer response than the other three tests probably because the specimen for this test was constructed from a different can of material. Test RDC62 was used as a "standard" for comparison in both the reverse strain-path test series and the strain-rate test series discussed later in this chapter.

Test results from undisturbed specimens following SP3A (UDC59, 60, and 61) are presented in Figure 3.21. Note the extreme anisotropy present in these specimens as evidenced by the measured strains at the end of HC loading (points B, Figure 3.21b). An isotropic specimen would have a principal strain difference value of zero at the end of HC. The three stress paths are qualitatively similar, despite the variability in the stress-strain data. Like the remolded specimens, the undisturbed specimens also exhibit a PCSD.

The results of typical remolded and undisturbed specimens following SP3A are presented in Figure 3.22 in order to (1) illustrate the variability of the undisturbed specimen data versus the variability of the remolded specimen data and (2) directly compare the remolded and undisturbed data. In comparison to the remolded test data, the test results from the undisturbed specimens exhibit extreme variability, in particular, the volumetric responses and the values of principal strain difference at the end of HC loading. The stressstrain curves of the undisturbed specimens are similar to those of the remolded specimens, however, the stress paths are somewhat different. The stress paths are again compared in Figure 3.23 with the CARES failure envelope superimposed upon the plot. All of the stress paths approach the failure envelope during the PCSD. These data clearly demonstrate the importance of conducting repetitive tests on remolded specimens in order to differentiate subtle changes in material response that might be difficult to discern within the inherent variability of "undisturbed" specimens.

Results of tests on undisturbed specimens UDC54, 55, and 58 following SP3C are presented in Figure 3.24. Test UDC55 developed a shear plane sometime during the strain-path loading, therefore, it should not be considered in the overall data analysis. The stress-strain curves and the stress paths of the two remaining tests, UDC54 and 58, are very similar despite their different volumetric responses during HC loading. Note that the PCSD is also present in these test data. These two tests are compared to tests on two 
remolded specimens (RDC31 and 32) in Figure 3.25. The one dramatic difference between the response of the remolded and undisturbed specimens is the repeatability in the remolded test data and the lack thereof in the undisturbed data. Quantitatively, the remolded specimens reached slightly higher values of principal stress difference. Figure 3.26 shows that the stress paths of both the remolded and undisturbed specimens reach the failure envelope; the data plates illustrate that the specimens enter the PCSD when the stress paths reach the failure envelope.

\subsubsection{SP2 Tests}

No undisturbed specimens were tested along the No. 2 strain paths; only remolded specimens were tested following strain paths $2 \mathrm{~A}, 2 \mathrm{~B}$, and $2 \mathrm{C}$. The stress paths for three remolded specimens following SP2A (RDC35, 36, and 37) are presented in Figure 3.27. The stress paths were qualitatively and quantitatively similar before peak stress difference was reached. The unloading stress paths show some variation which is attributed to the inconsistent movement of the individual strain paths around the corner of the intended strain path (see data plates). The reduction in mean normal stress at the start of strain-path loading was necessary to bring the strain path down to the desired path. The stress-strain curves and volumetric responses are identical for all three specimens. Unlike the stress paths from the No. 3 strain-path tests, these stress paths do not approach the failure envelope (Figure 3.27a).

Results from three of the four tests following SP2B (RDC24, 26 and 27 , Figure 3.28) exhibit identical stress and strain responses. During test RDC25, equipment problems developed, which could have affected the data. Note that none of the strain paths from these tests were able to reach the point of lateral strain reversal (Figure 3.28d); there was not enough elastic response in the specimens to rebound sufficiently. In the FY 83 test program, all of the specimens following SP2B were able to reach that point.

The results of three tests following SP2C are presented in Figure 3.29. The smaller strains of SP2C made the effect of the increasing lateral strains at the start of loading quite noticeable. This contributed to the relatively large variability present in the test data. Also note the extreme movement of the measured strain path of specimen RDC 49 away from the intended path; this caused the large "bulge" in the stress path at and after the point of peak 
stress difference. This series of data does not display the repeatability observed in the majority of the data reported herein.

\section{6 REVERSE SP TESTS}

In this series of tests, one stress path (generated by test RDC62) was followed three times, producing three measured strain paths. The only section of each test that varied in test procedures was the PCSD. Through this section of the stress path, the confining pressure was reduced at different rates while still following the desired stress path. Some knowledge of earlier test data was required in order to determine when the specimen had reached the PCSD.

Figure 3.30 presents the data from the three reverse tests, RDC63, 64, and 67; test RDC62 served as the standard for comparison. Note the slight deviation of the stress path of test RDC63 (Figure 3.30a). The reduction in confining pressure was initiated too early and too quickly, and the result was an increase in principal stress difference. Posttest analysis of the stressstrain data from test RDC63 (Figure 3.30b) indicated that the point at which the confining pressure was reduced lay just outside the PCSD. The times through the PCSD were obtained for each test from Figure 3.31; these values were $1.2,4.6,9.9$, and 10.4 minutes for tests RDC64, 63, 67, and 62, respectively. With the exception of the one excursion in the stress path of test RDC63, all of the stress paths plot on top of each other. The excellent duplication of the stress paths is further demonstrated by the stress-strain curves from all four tests in that they are identical up to the point of peak stress difference (if the axial strains are re-zeroed at the end of HC loading). As encountered in the FY 83 test program, a non-unique relationship between stress and strain was discovered after completing these reverse tests. It is obvious that the material exhibits strong time-dependent behavior during the PCSD, which only occurs during the time the stress paths are in the proximity of the failure envelope.

\subsection{STRAIN-RATE TESTS}

Figure 3.32 presents the results from five tests (RDC62, 65, 66, 68, and 72) which were conducted in order to discern possible strain-rate effects while following a given strain path, in this case SP3B. As in the reverse strain-path tests, test RDC62 served as the standard test for comparison; it was completed in approximately 30 minutes. Tests RDC65, 66, and 68 were 
completed in times of $17.6,5.3$, and 5.2 minutes, respectively. The fifth test, RDC72, was terminated prior to completion; it would have been completed in approximately 60 minutes. Data from the three "slow" tests (greater than 17 minutes to complete the strain path) exhibit negligible differences in their stress-strain responses, i.e., no rate effects were observed (Figure 3.32). The two "fast" tests (approximately 5 minutes to complete the strain path) have a noticeable increase in peak stress difference. Some of this increase in stress difference can be attributed to the overshoot in the measured strain path when going around the corner of the intended strain path. However, if one compares the slow and $\mathrm{f}$ ast tests at corresponding points on the strain path before the corner, the faster tests exhibit a definite increase in principal stress difference. Also note that in Figure $3.32 a$, the initial stress paths for the fast tests are close to a $3: 1$ slope (TXC 1oading). In comparison, the slow tests required a decrease in mean normal stress, even though the measured strain paths for the fast and slow tests are identical up to the corner of the intended strain path (start of the $1: 1$ ). After considering all of the variables in the data, one should conclude that there is a slight increase in peak stress difference due to strain-rate effects.

\subsection{EFFECTS OF CONF INING PRESSURE}

All of the response data from the SP 3 tests indicate that the PCSD occurs when the stress path is in the proximity of the failure envelope. With this knowledge, a method of reliably determining the compression failure envelope is available. Several strain-path tests could be conducted at different initial confining pressures, the PCSD determined in stress space, and the loci of these points plotted in order to define the failure envelope. This method has several advantages over conventional methods: (1) "failure" in these strain-path tests is distinct, the PCSD is clearly evident in all of the SP3B and SP3C stress-strain curves, and (2) the PCSD can be achieved at much smaller magnitudes of strain, both axial and radial, thus maintaining more uniform stresses within the test specimens. This method of defining failure could be used as opposed to an arbitrary definition of failure, e.g., 15- or 20-percent axial strain during TXC loading.

In order to prove that this method for determining a failure envelope is a viable alternative, three strain-path tests, RDC69, 70, and 71, were conducted following SP3B. These three tests were subjected to initial prestresses 
of $3.45,1.72$, and $0.41 \mathrm{MPa}$, respectively. Test RDC62 provided a fourth test at a prestress level of $6.9 \mathrm{MPa}$. Results from these four tests are presented in Figure 3.33. As illustrated in Figure 3.33d, the repeatability of the strain paths is excellent; differences are only distinguishable at the start of loading. The stress-strain and volume-strain curves are qualitatively similar for all four tests. The PCSD was achieved in all four tests. The major points of interest lie in the stress-path data. Note the dramatic change in the stress path of specimen RDC71 (Figure 3.33a and 3.34); its unloading stress path crosses over the loading path. This response is similar to that of overconsolidated clays. For this test, the prestress level of 0.41 MPa was probably less than the stresses imposed upon the specimen during construction; thus the specimen was in reality "overconsolidated." The stress paths during the PCSD fall along the failure envelope (Figure 3.34). It is obvious that several strain-path tests conducted at different initial confining pressures will adequately define a compression failure envelope.

\subsection{SP1T AND $2 \mathrm{~T}$}

Eight strain-path tests were conducted on paths $1 \mathrm{~T}$ and $2 \mathrm{~T}$ in the FY 84 test program. Specimens following SP1T were prestressed to $0.41 \mathrm{MPa}$. The $2 \mathrm{~T}$ strain-path tests were conducted at two different levels of prestress, 0.41 and $3.45 \mathrm{MPa}$.

Results from tests UDC73, 74 , and 80 following SP1T are presented in Figure 3.35. The data from all three tests are qualitatively the same but quantitatively different. The stress paths are especially interesting; each path exhibited a reduction in mean normal stress with increasing values of principal stress difference while at the same time the strain path went around the corner. Past this point, the stress paths again continued to move to higher values of both principal stress difference and mean normal stress. Lade (Reference 4) reports a similar response for undisturbed CARES specimens subjected to SP1T. As shown in Figure 3.36, the stress paths for all three tests inove parallel to the failure envelope as mean normal stress increases.

At the point of mean normal stress decrease, there exists a state of increasing axial strain, decreasing radial strain, near constant (uniform method) or increasing (cone method) volumetric strain, and increasing principal stress difference. This combination of responses implies that shear induced volume change occurred. This can be supported by data from some of 
the other strain-path tests in which relatively little change in mean normal stress during the initial strain-path loading occurred and yet compressive volumetric strains were calculated using both calculation methods, e.g., RDC21 (Figure 3.18).

SP2T test results with a prestress of $0.41 \mathrm{MPa}$ (UDC75, 76, and 77 , Figures 3.37 and 3.38 ) show significant differences in response. These differences are attributed to the variations in specimen water content and density. Note especially specimen UDC75 which had a water content greater than 8 percent, a low peak stress difference, and a large volume change. SP2T test results with a prestress of $3.45 \mathrm{MPa}$ (UDC7 8 and 79 , Figure 3.39) exhibit extreme differences in response; these differences are again attributed to the unusually high water content $(>8 \%)$ and thus the lower value of dry density of specimen UDC78. UDC78 achieved lower values of peak stress difference and larger volumetric strains during prestress than specimen UDC79. The stress paths from these two tests are presented in Figure 3.40. In observing these test results, one is again reminded of the variability of the undisturbed specimens and the importance of conducting remolded tests in order to obtain basic response information. 


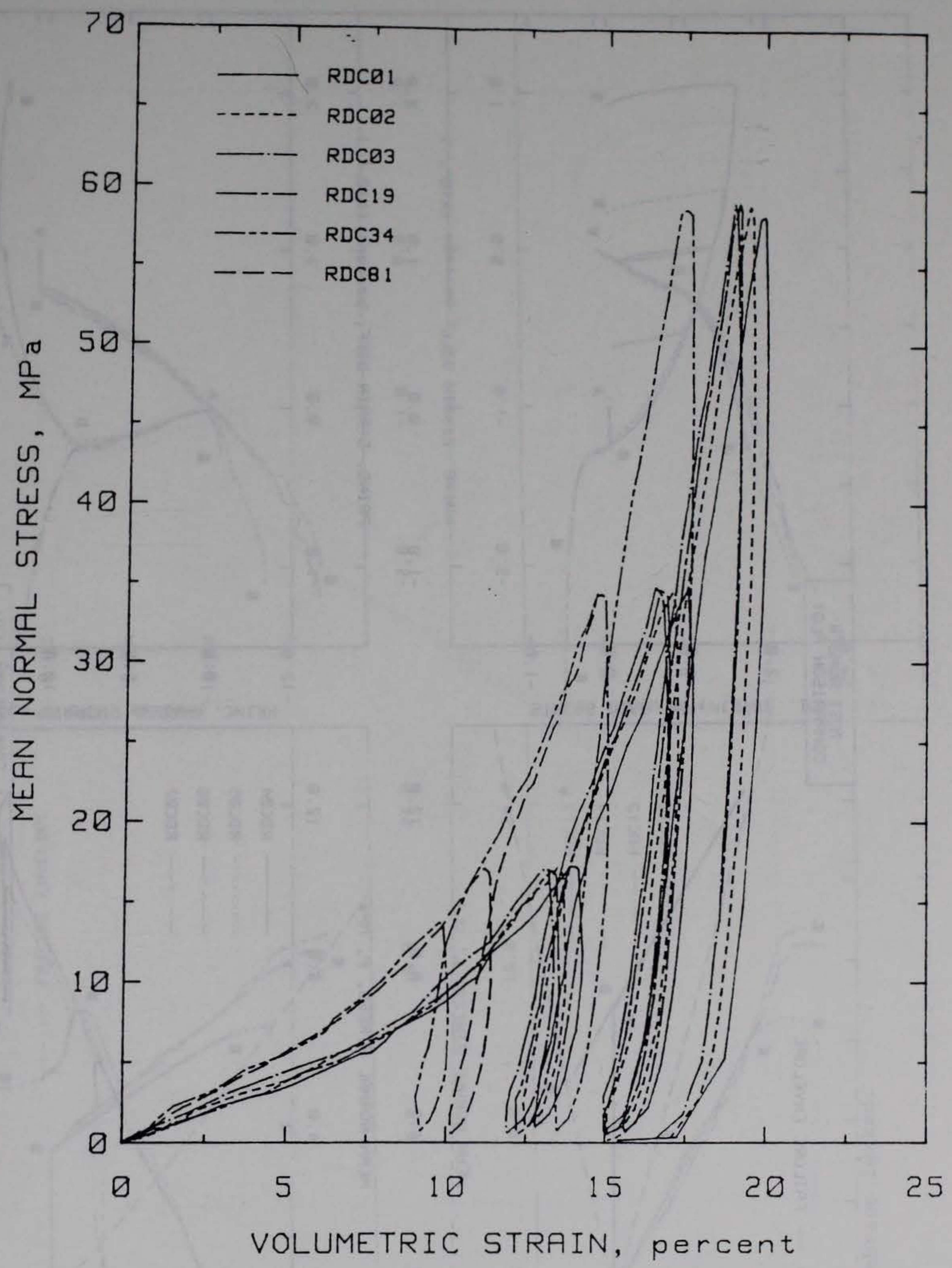

Figure 3.1. Static high-pressure hydrostatic compression test results. 


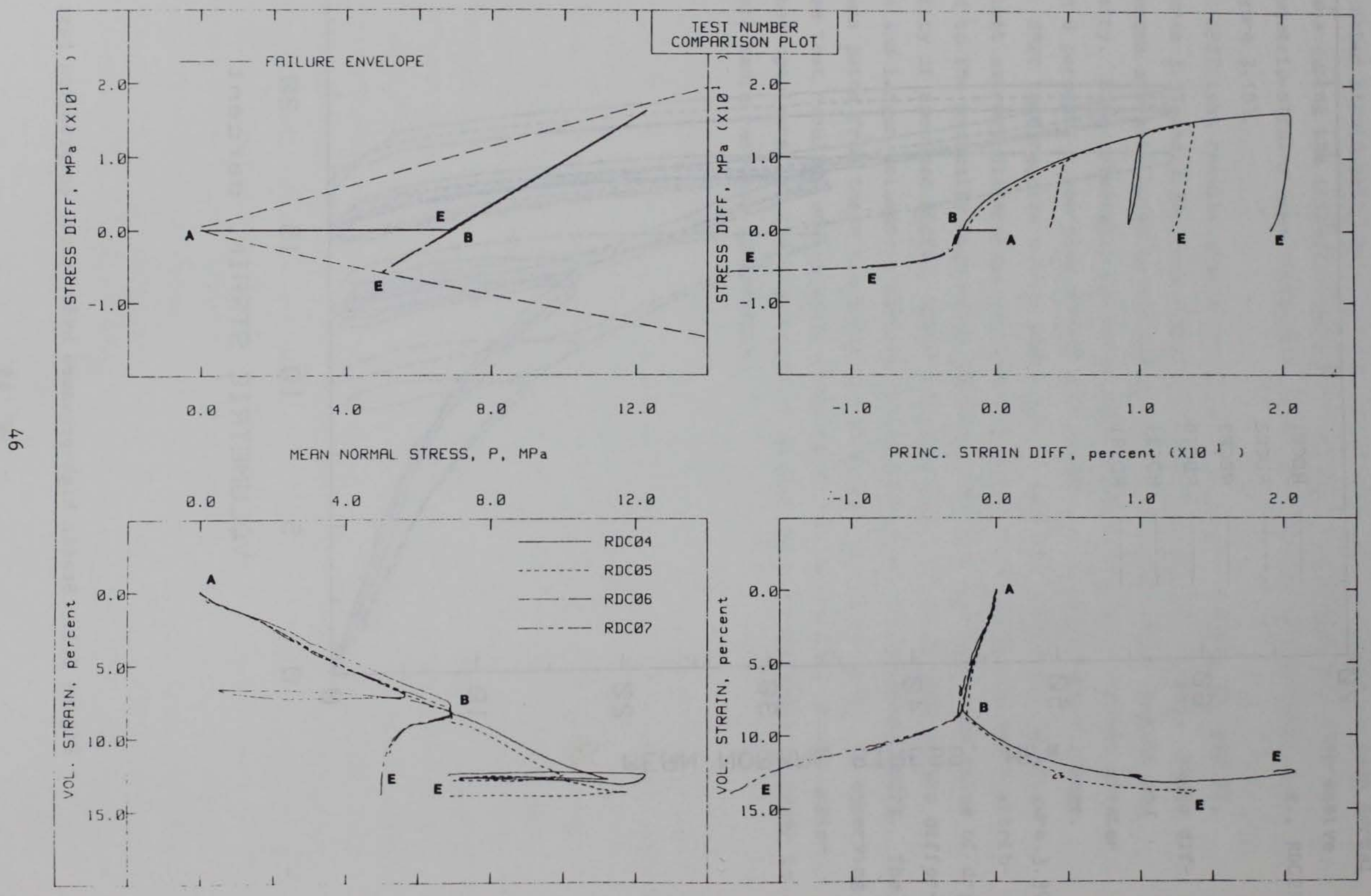

Figure 3.2. CTC and RTE test results. 


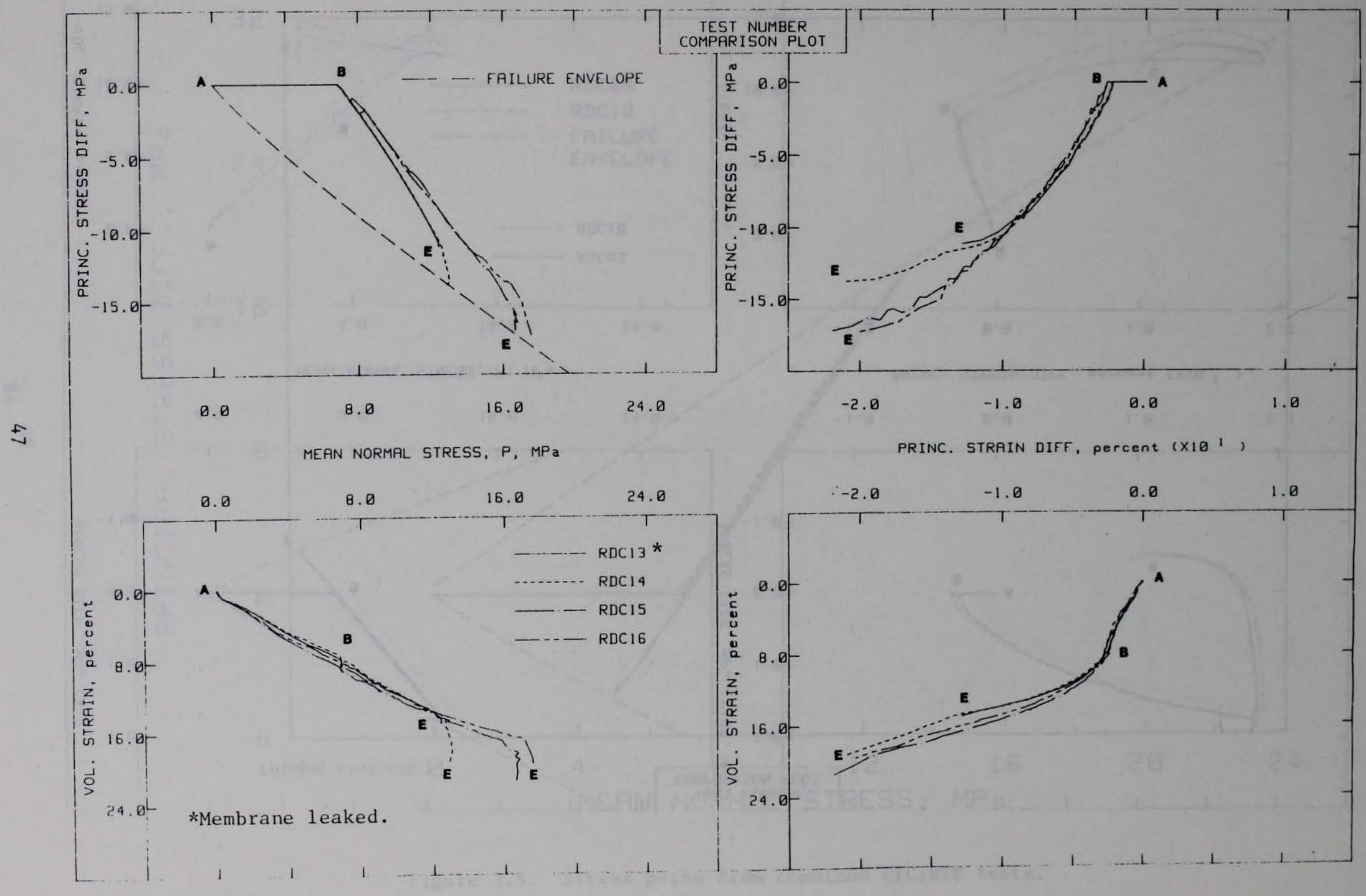

Figure 3.3. CTE test results. 


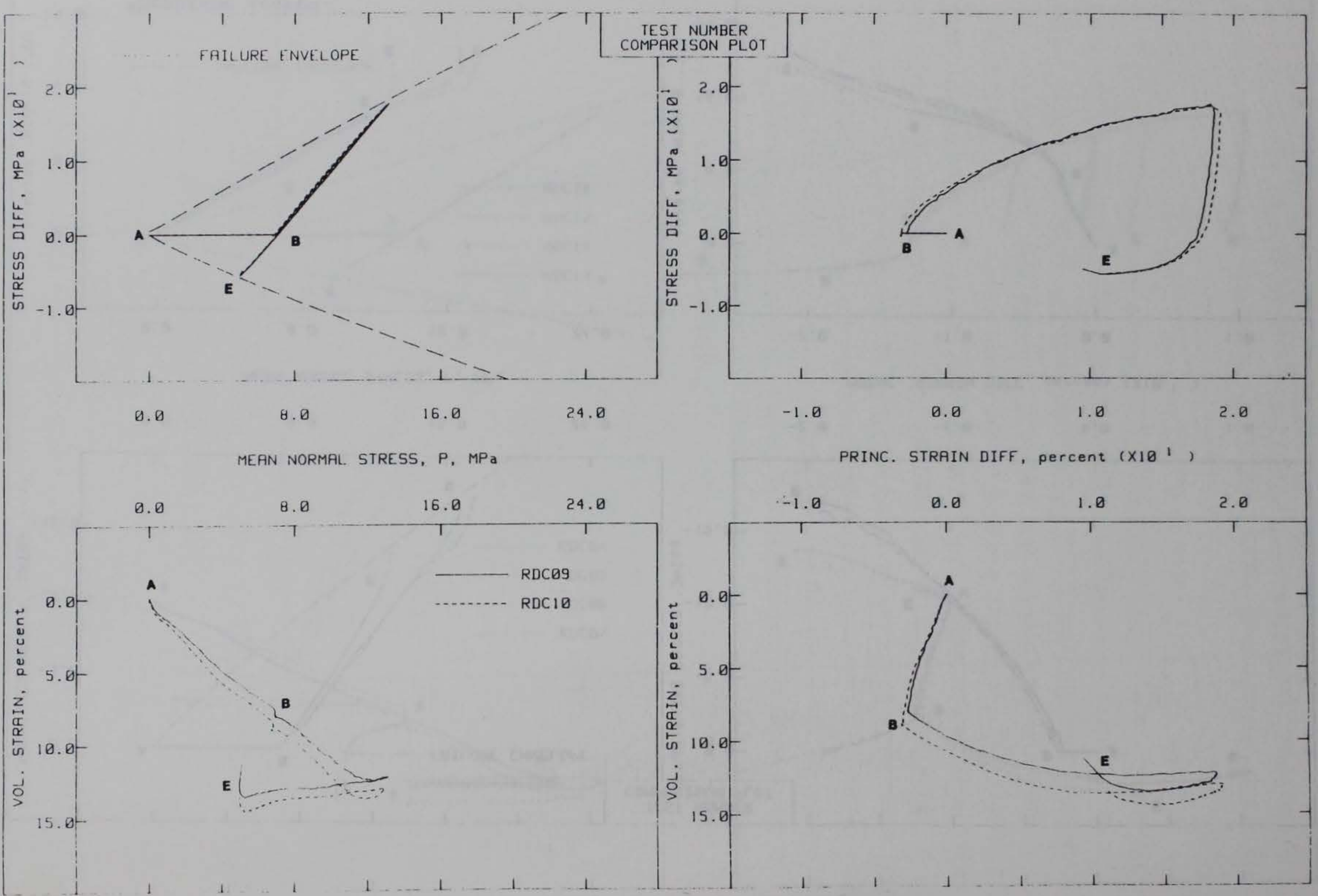

Figure 3.4. Combined CTC/RTE test results. 


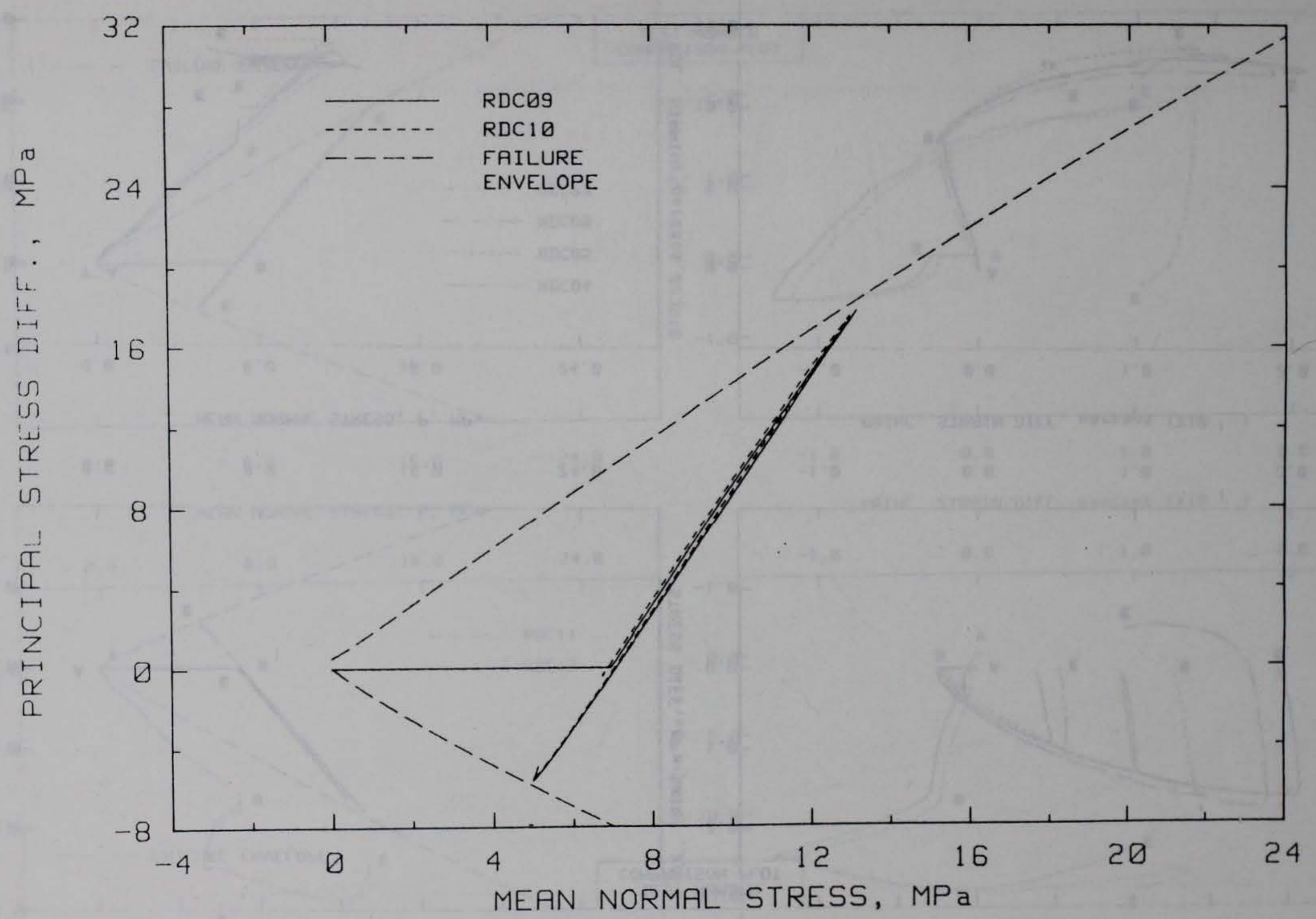

Figure 3.5. Stress paths from combined CTC/RTE tests. 


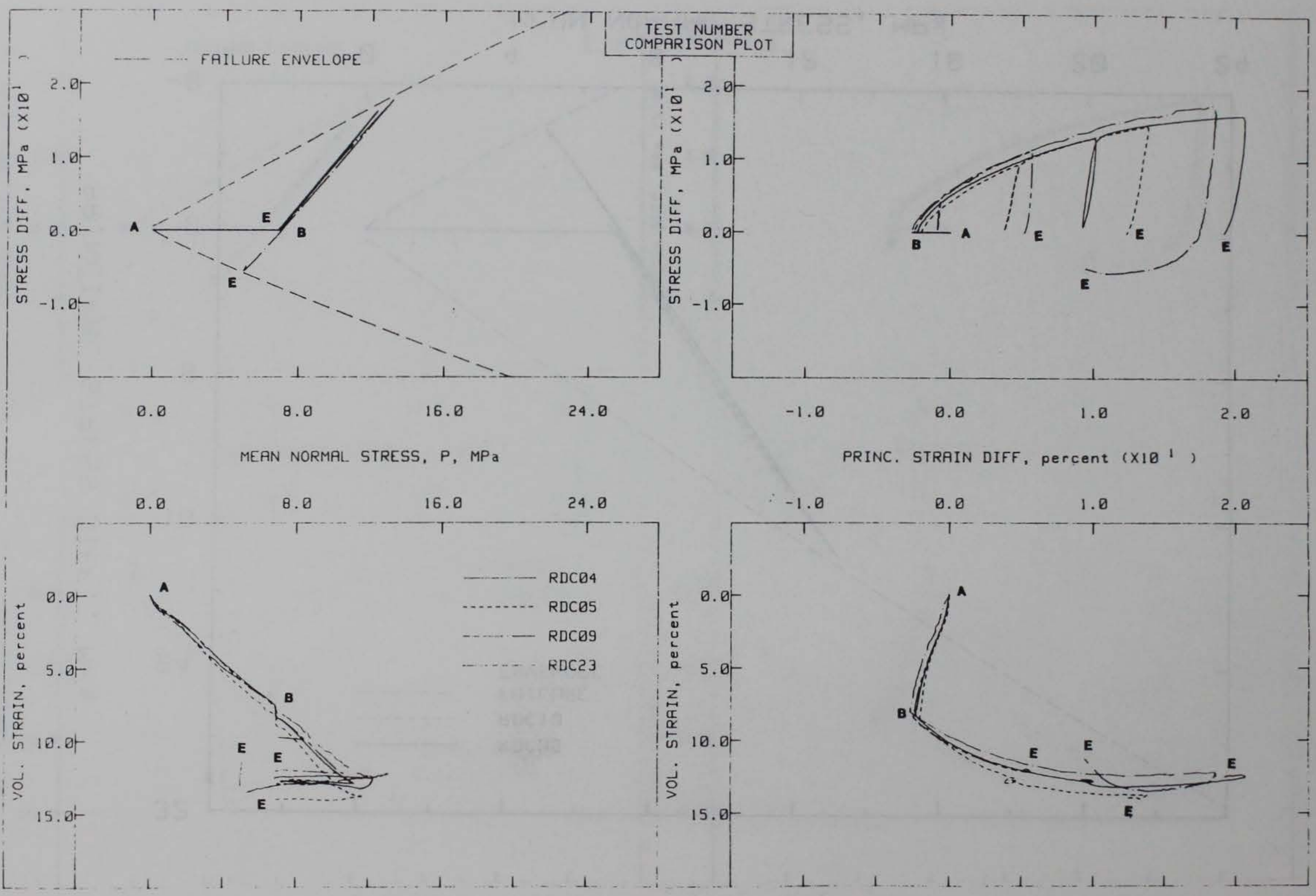

Figure 3.6. Comparison of CTC and CTC/RTE test results. 


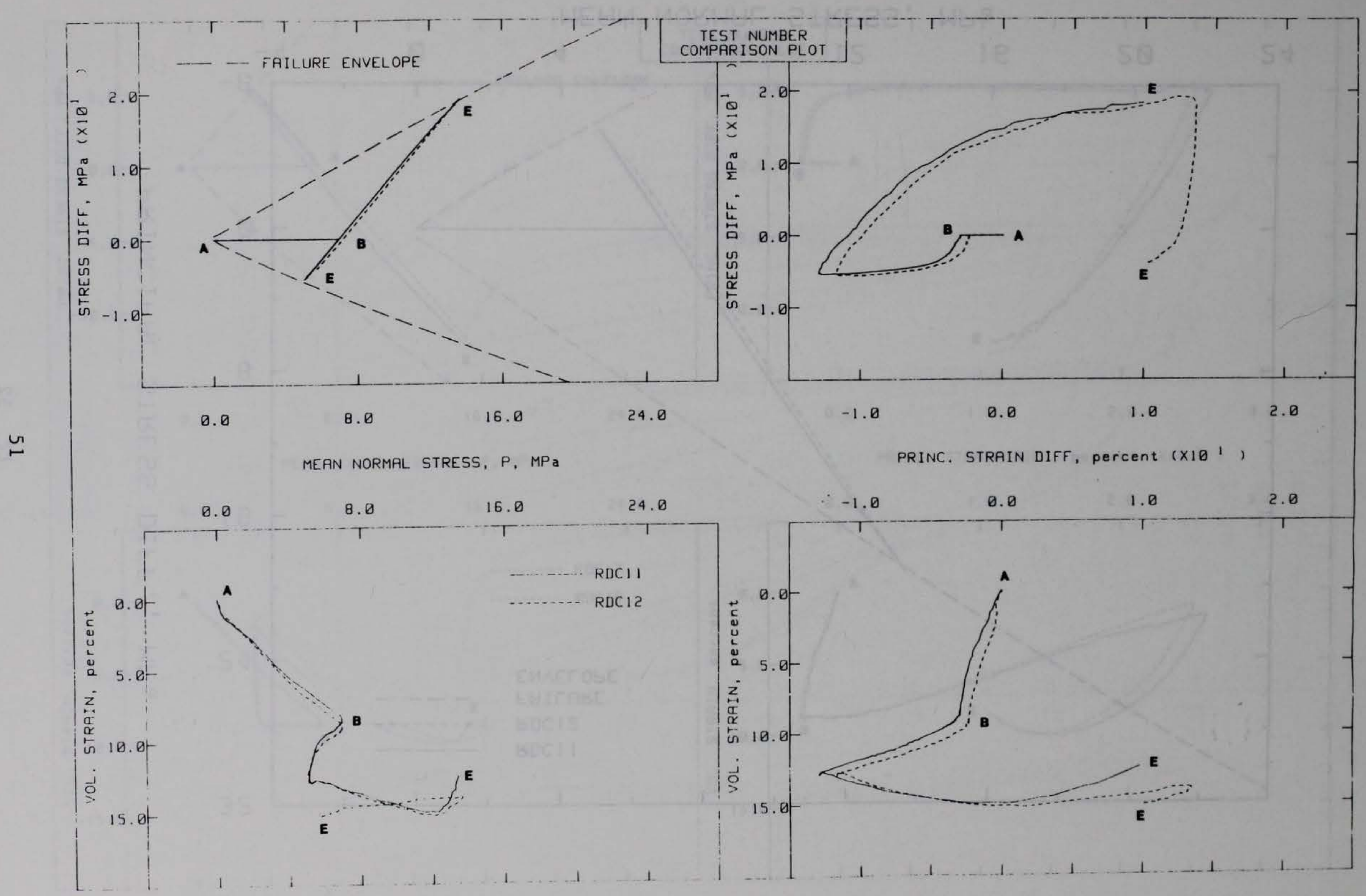

Figure 3.7. Combined RTE/CTC test results. 


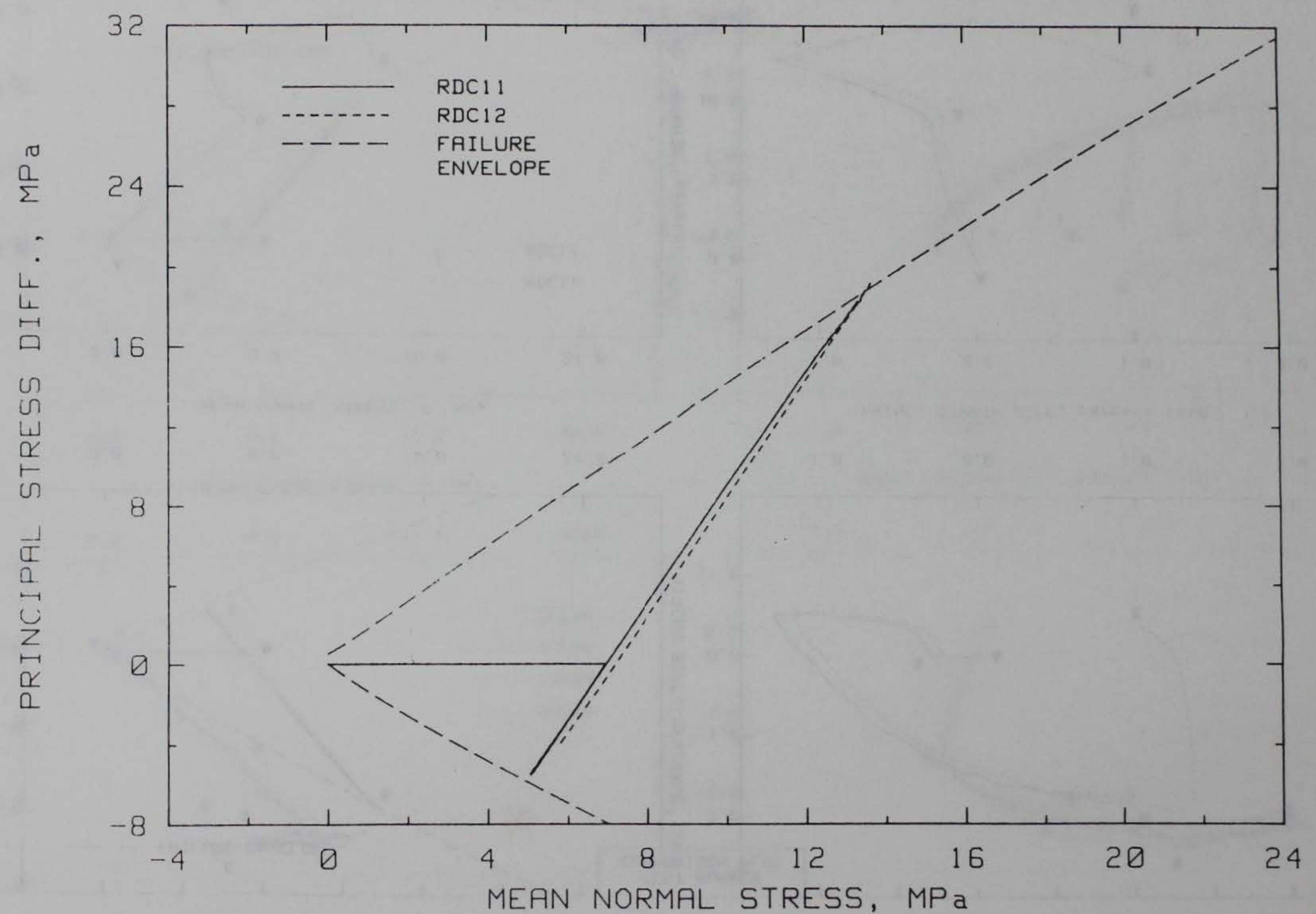

Figure 3.8. Stress paths from combined RTE/CTC tests. 


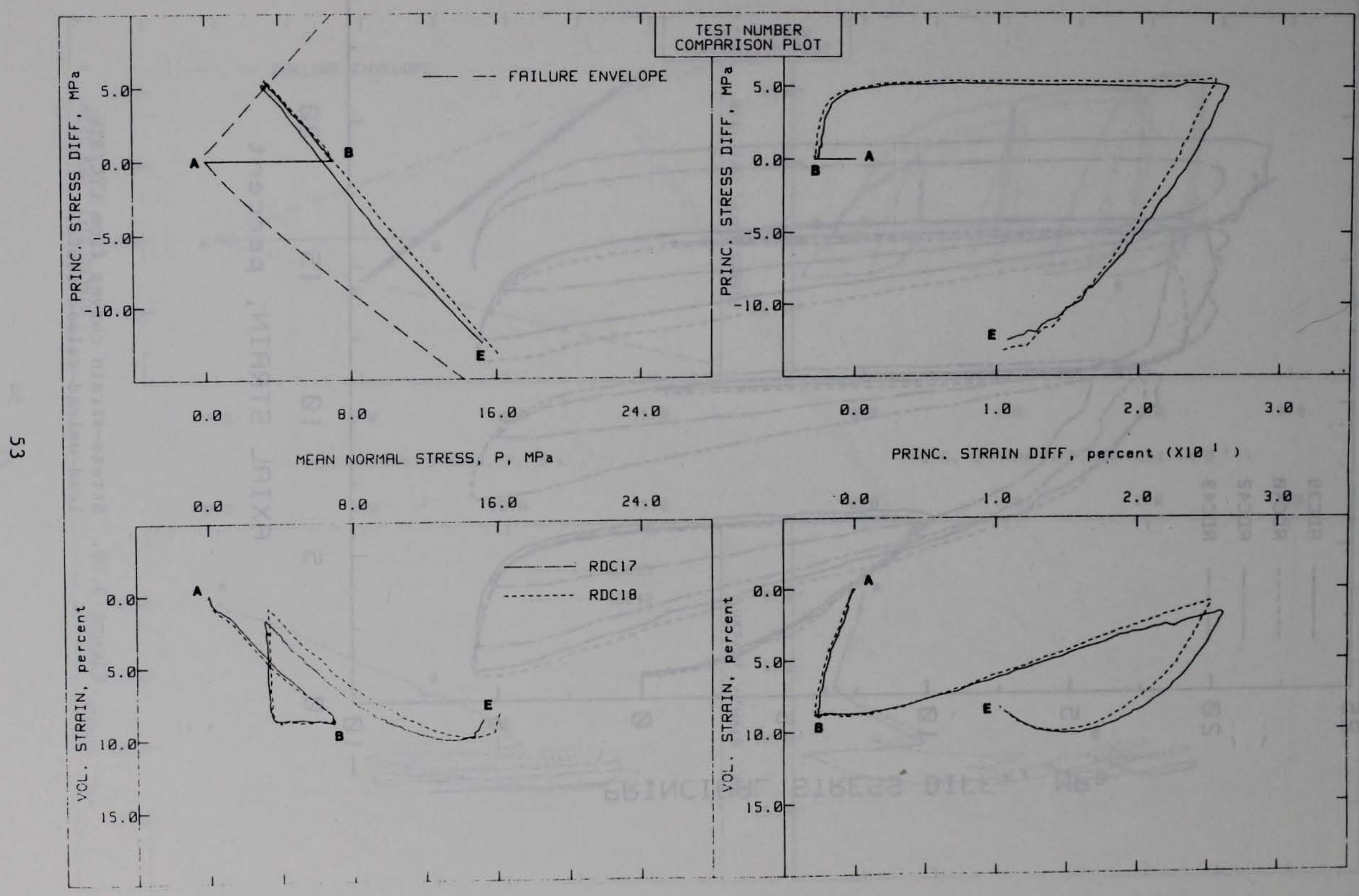

Figure 3.9. Combined RTC/CTE test results. 


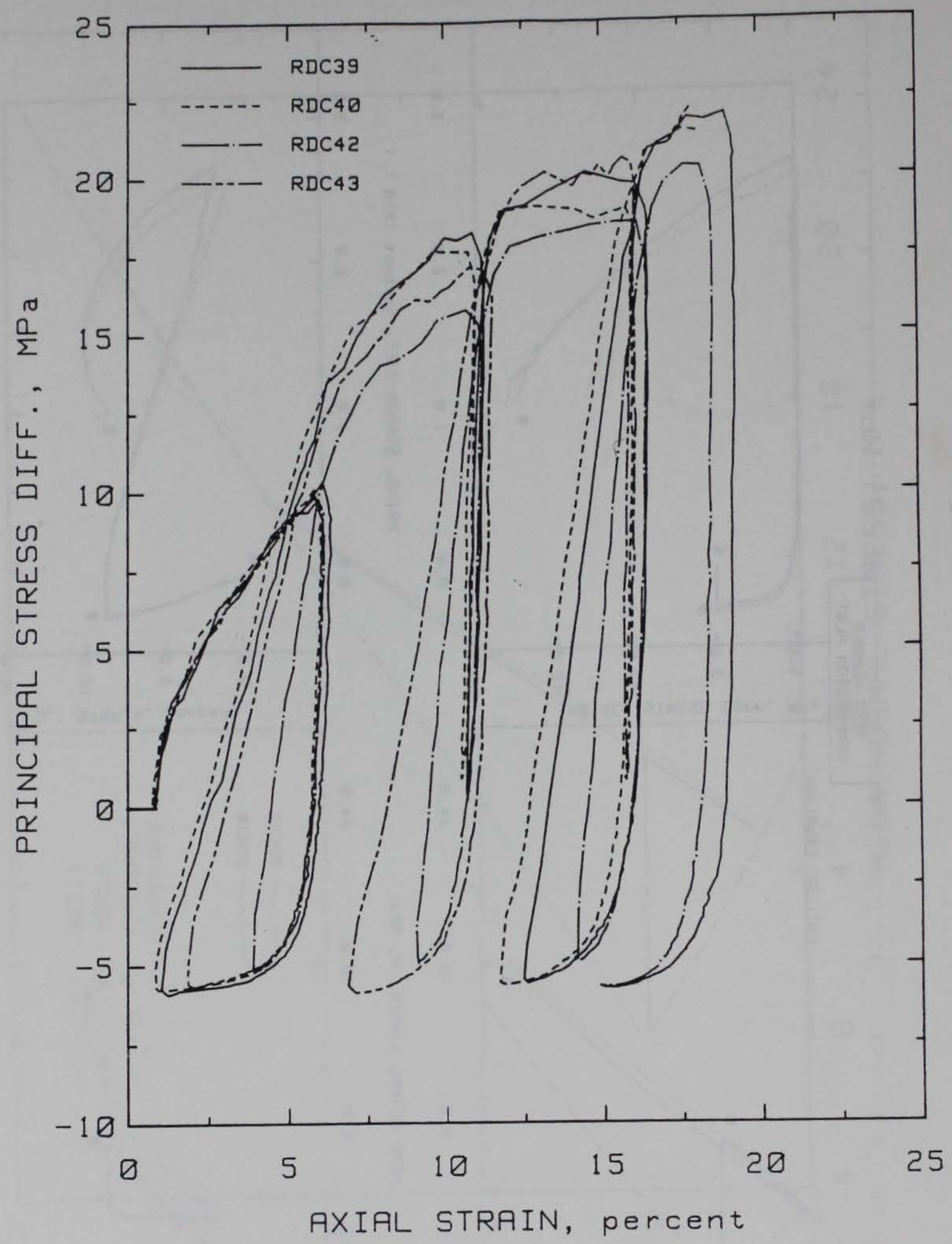

Figure 3.10. Stress-strain curves from CTC/RTE load-unload-reload tests. 


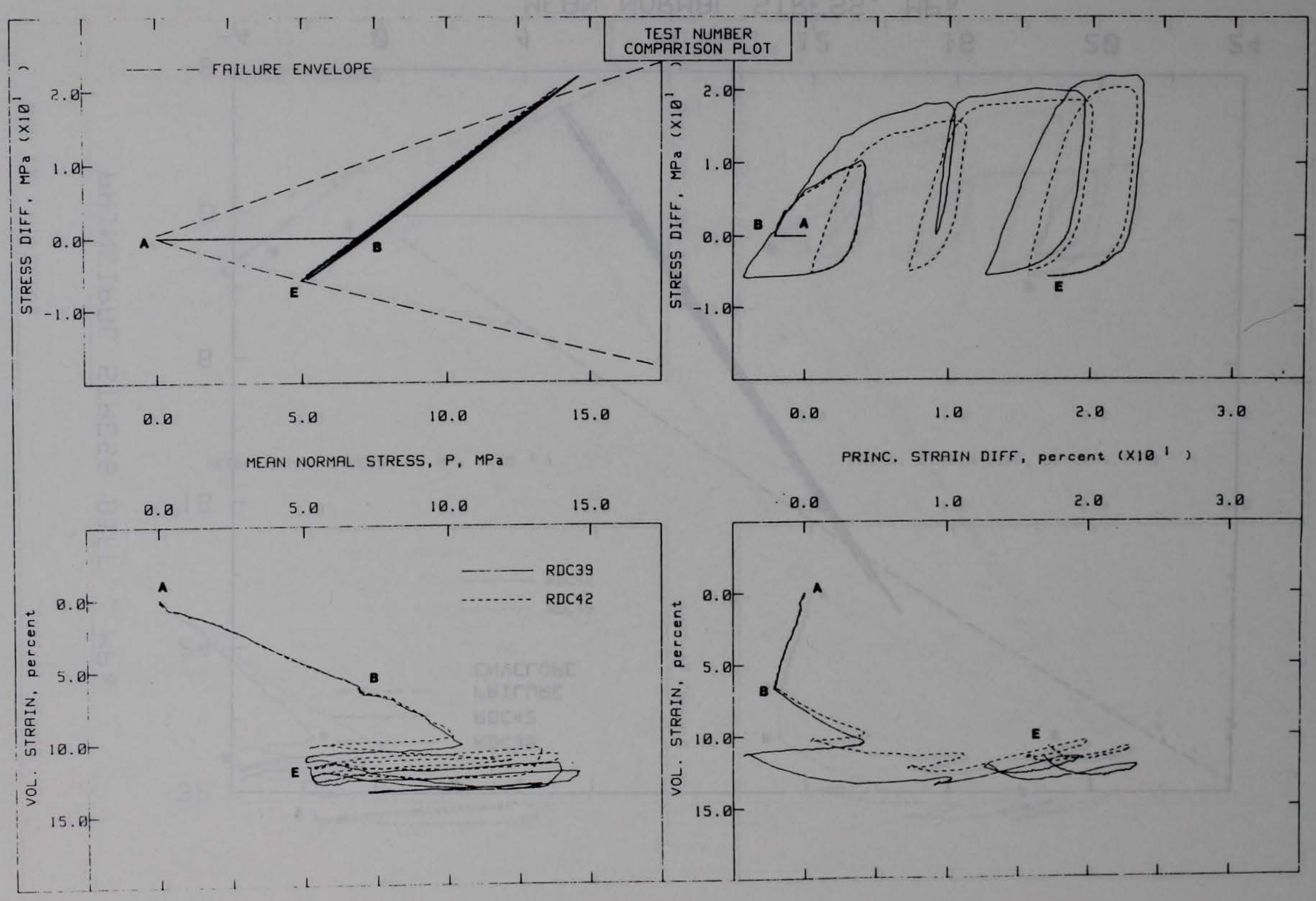

Figure 3.11. Multiple unload-reload CTC/RTE test results at $6.9 \mathrm{MPa}$ prestress. 


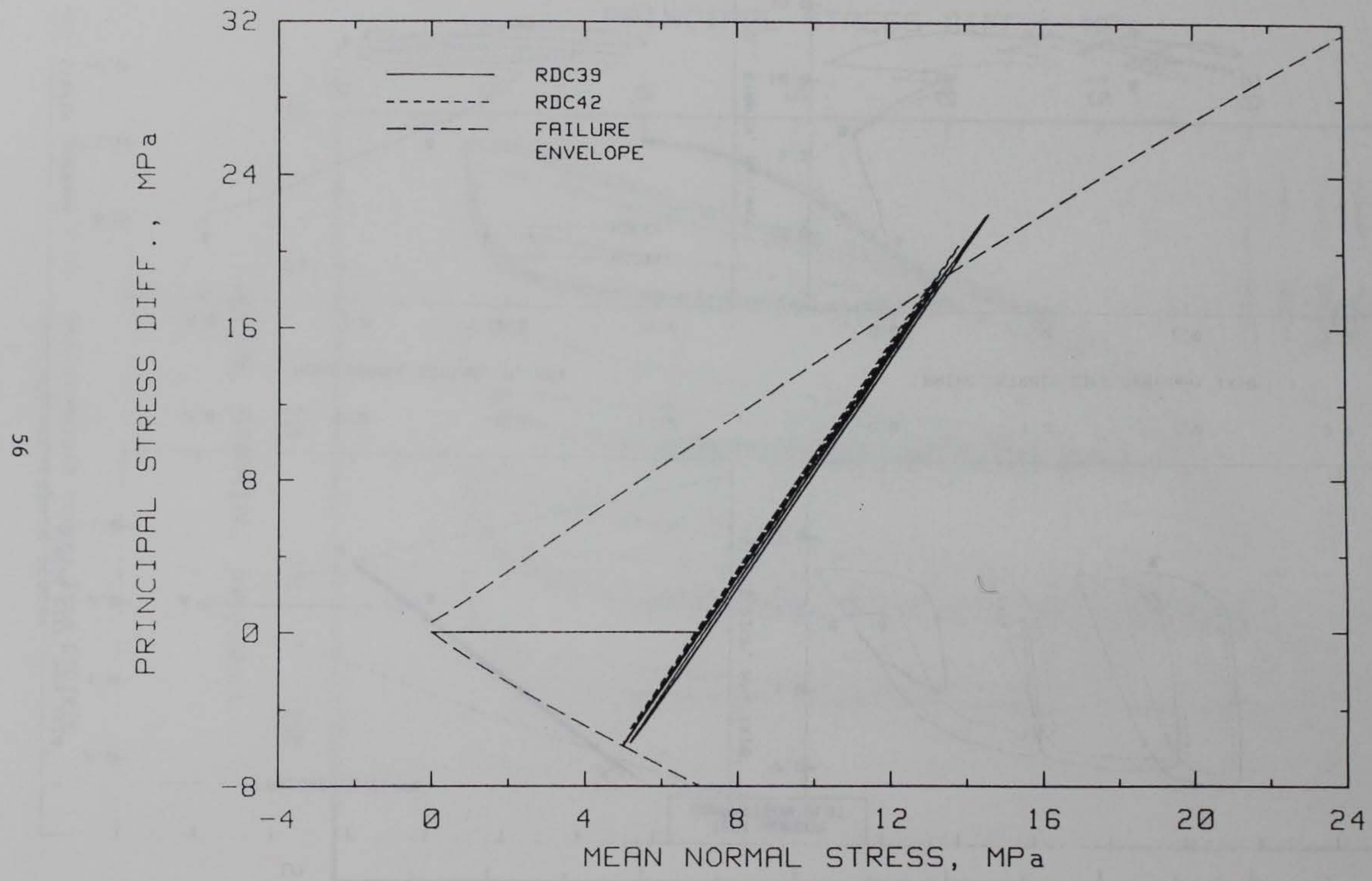

Figure 3.12. Stress paths from unload-reload CTC/RTE tests at $6.9 \mathrm{MPa}$ prestress. 


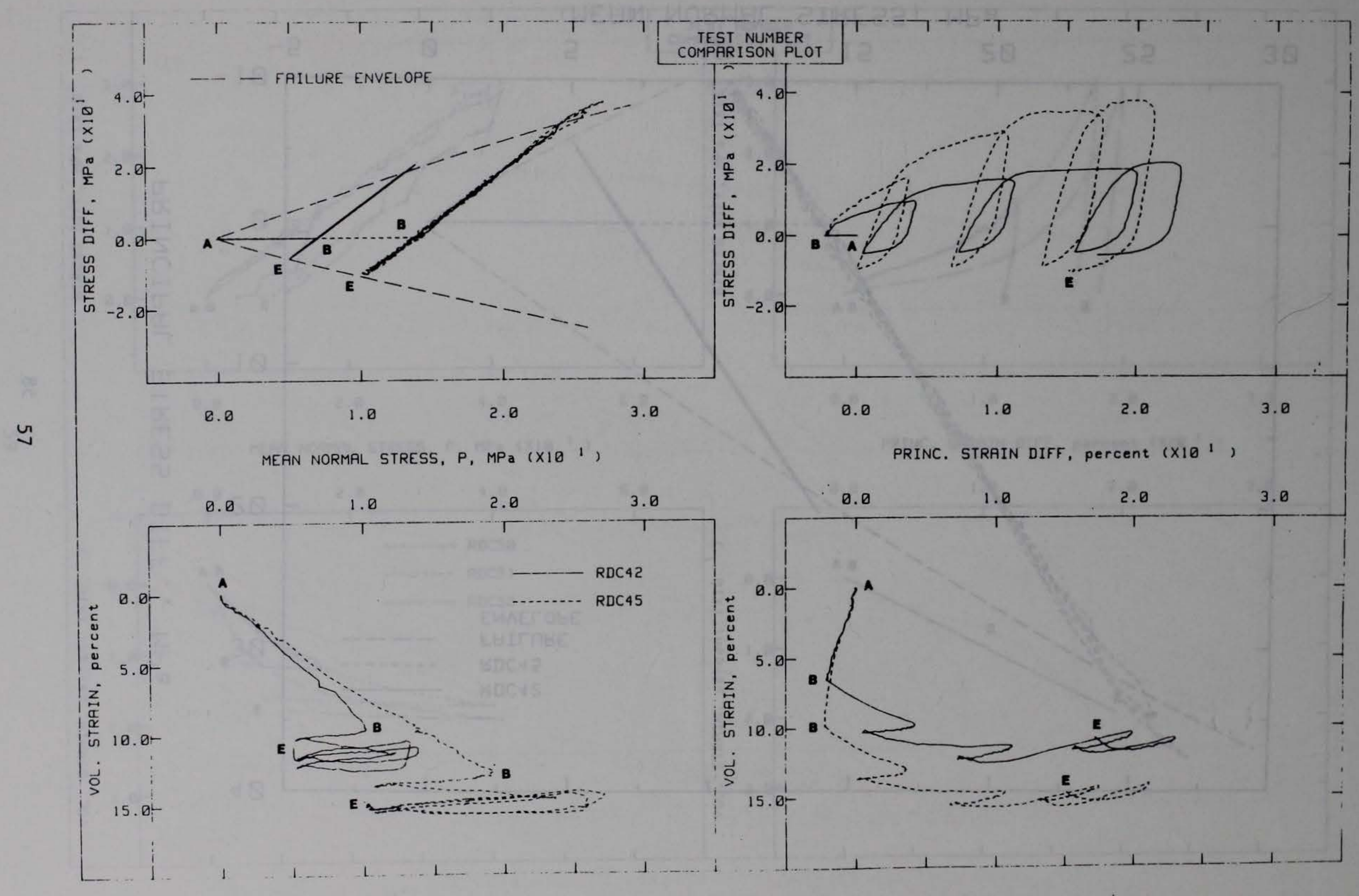

Figure 3.13. Comparison of unload-reload CTC/RTE tests at 6.9 and $13.8 \mathrm{MPa}$. 


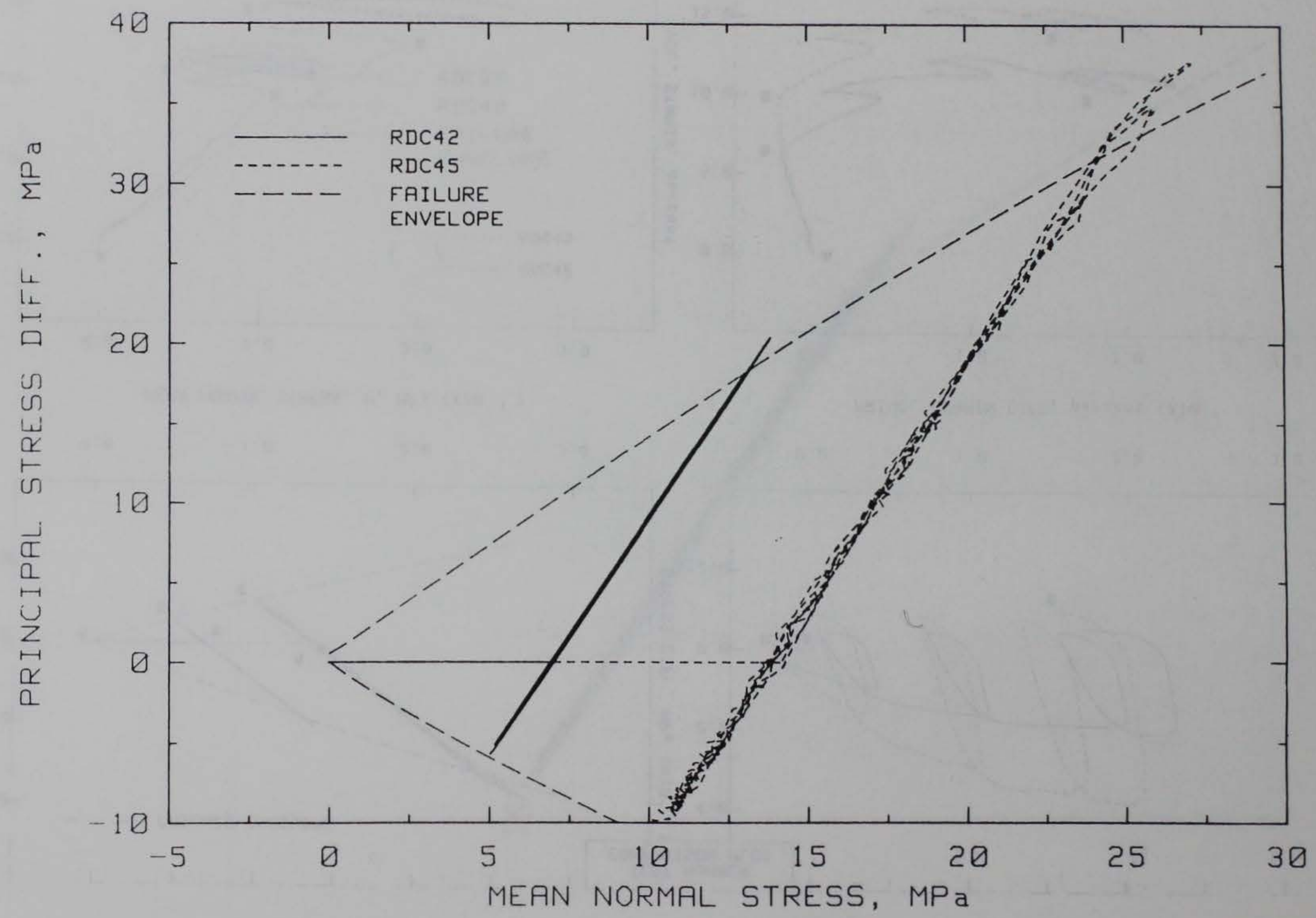

Figure 3.14. Stress paths from unload-reload CTC/RTE tests at 6.9 and $13.8 \mathrm{MPa}$. 


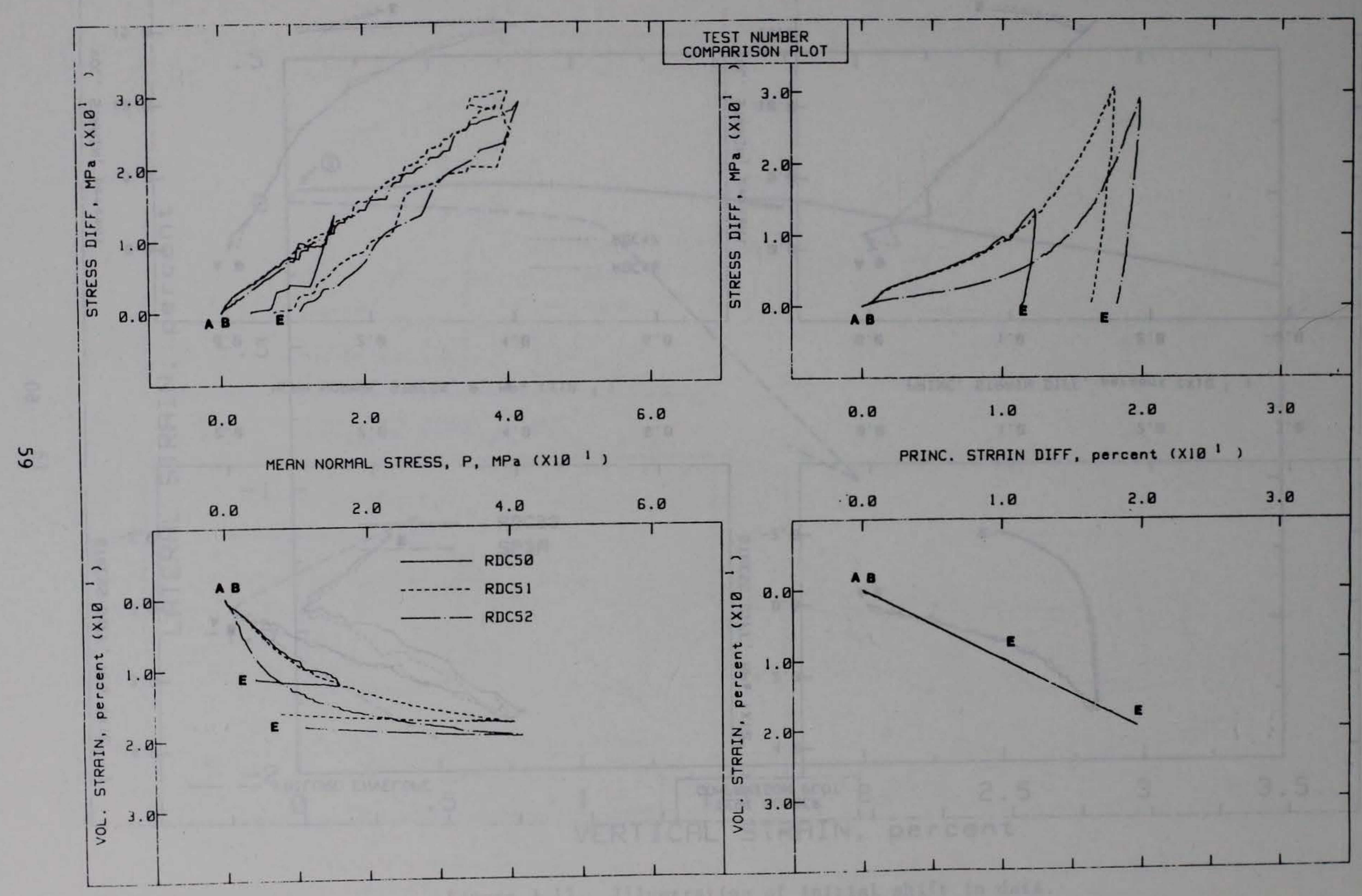

Figure 3.15. $\mathrm{UX} / \mathrm{K}_{\mathrm{o}}$ test results. 


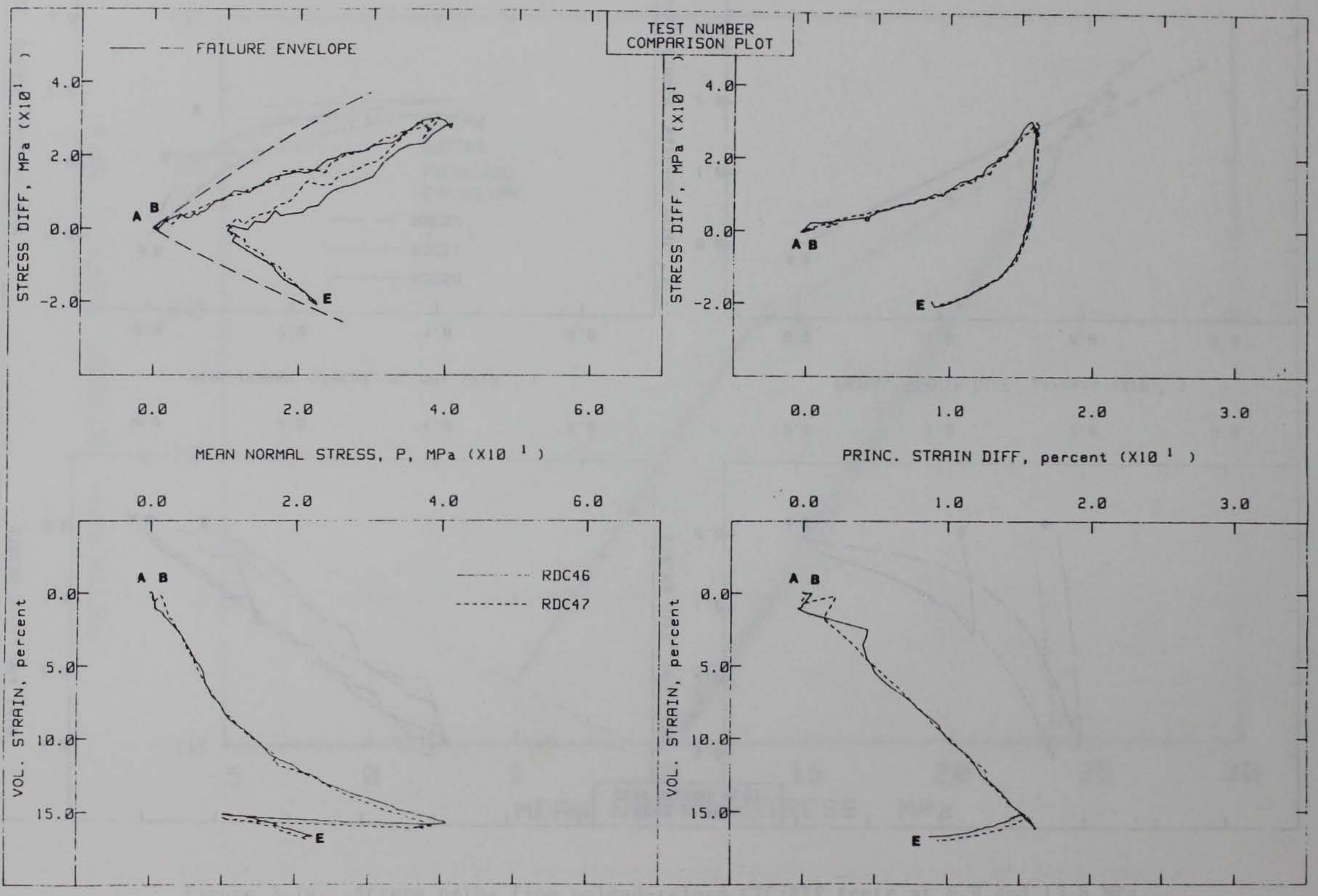

Figure $3.16 . \mathrm{K}_{\mathrm{o}} / \mathrm{CTE}$ test results. 


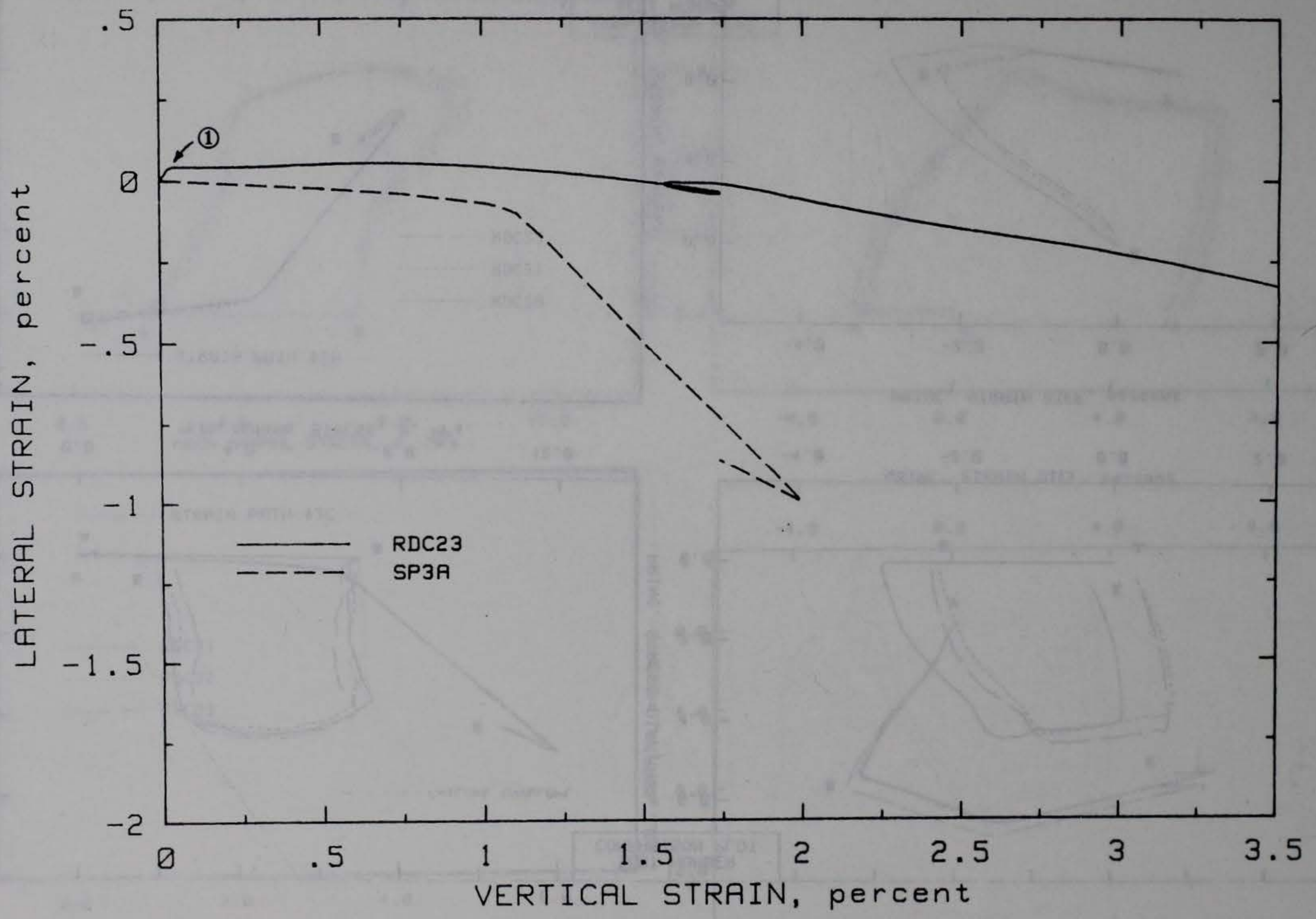

Figure 3.17. Illustration of initial shift in data. 


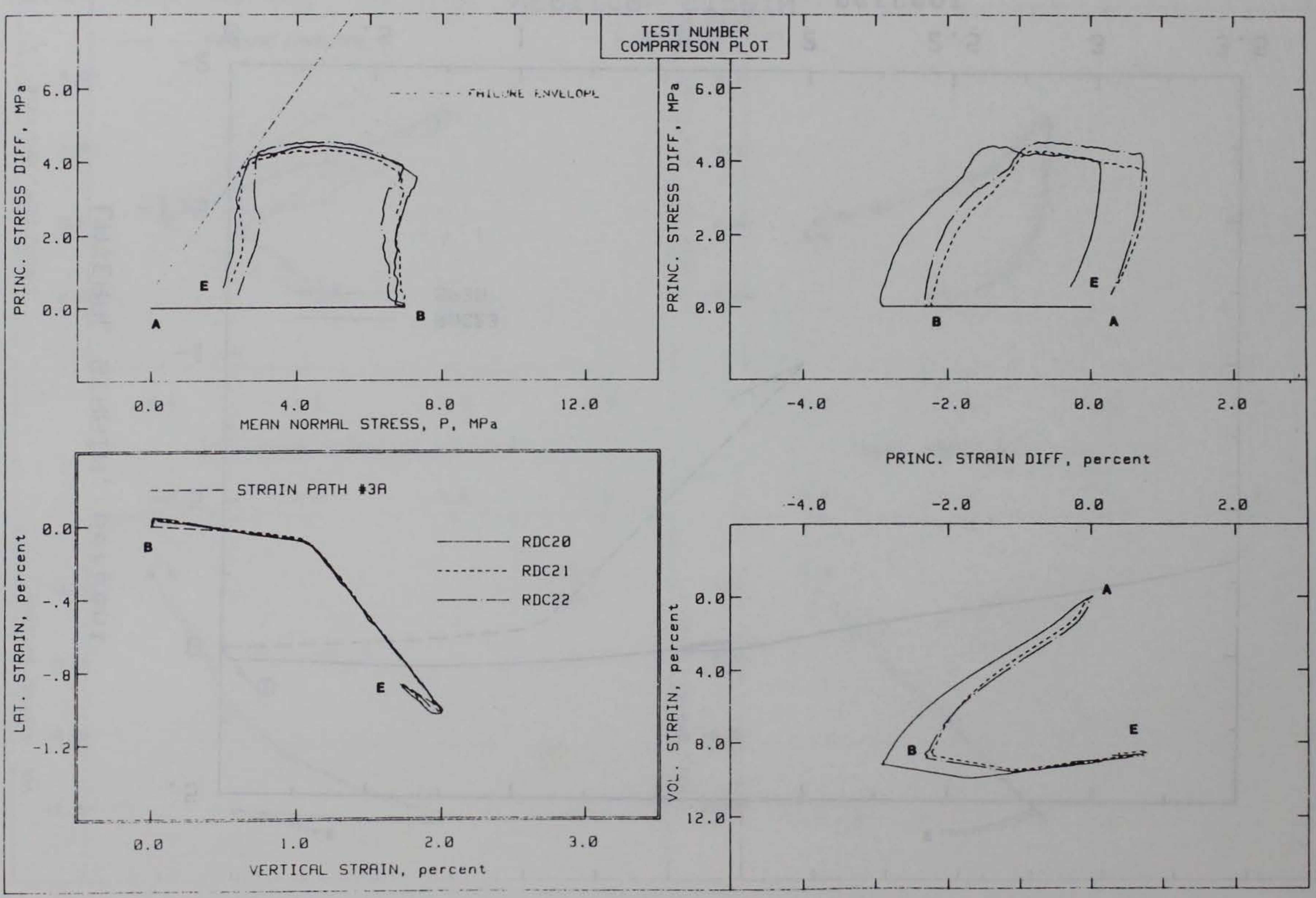

Figure 3.18. Strain path 3 A test results from remolded specimens. 


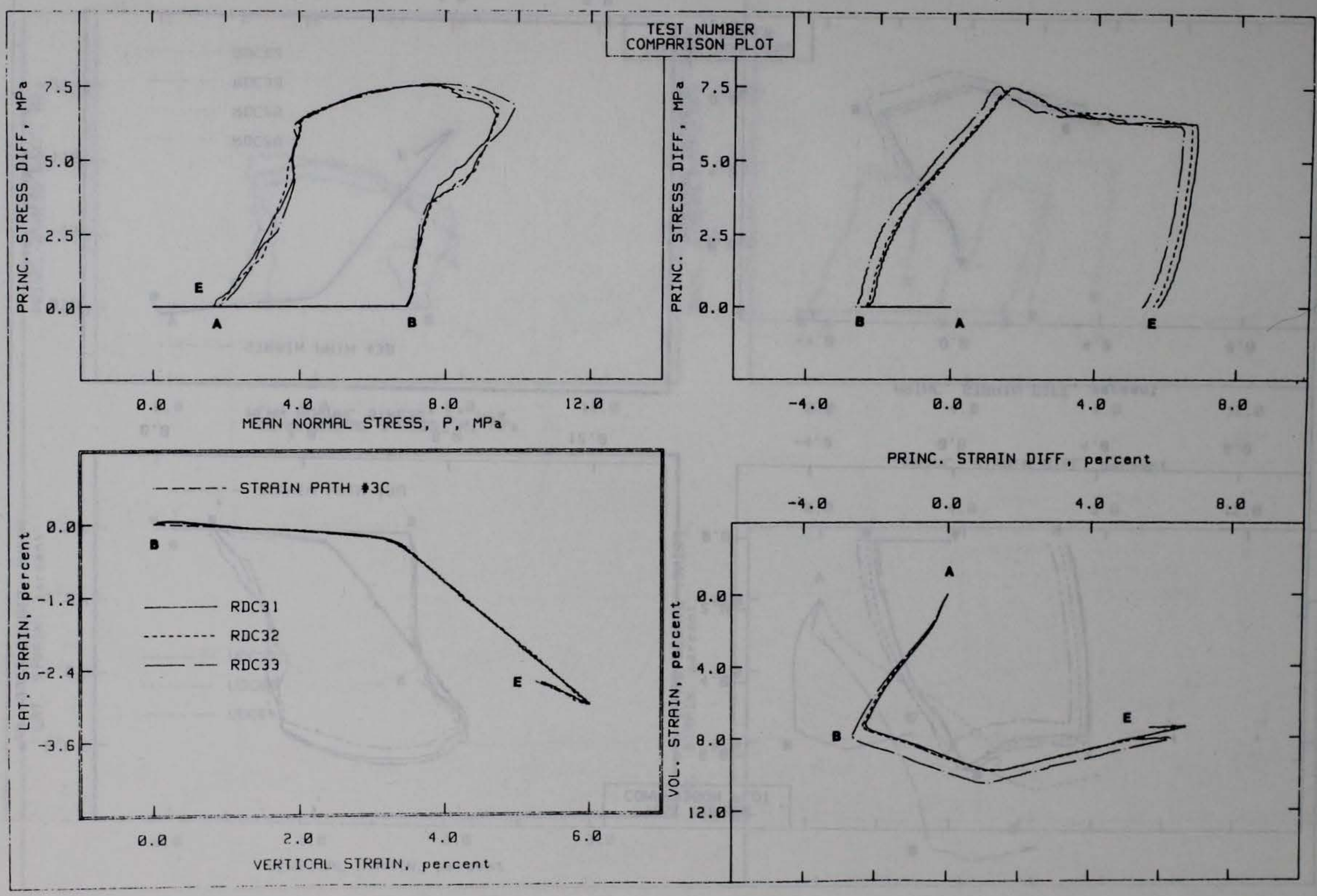

Figure 3.19. Strain path $3 C$ test results from remolded specimens. 


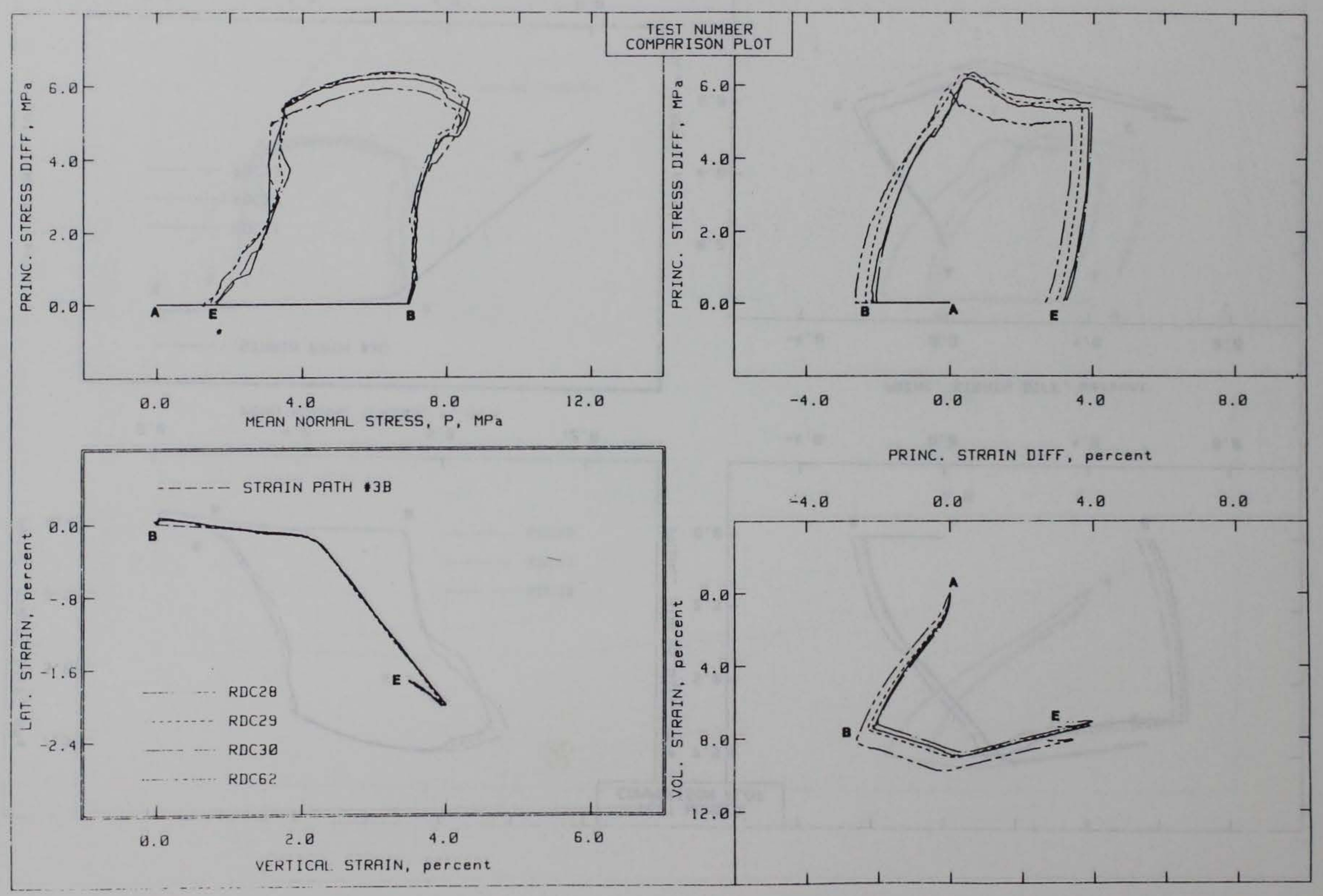

Figure 3.20. Strain path 3 B test results from remolded specimens. 


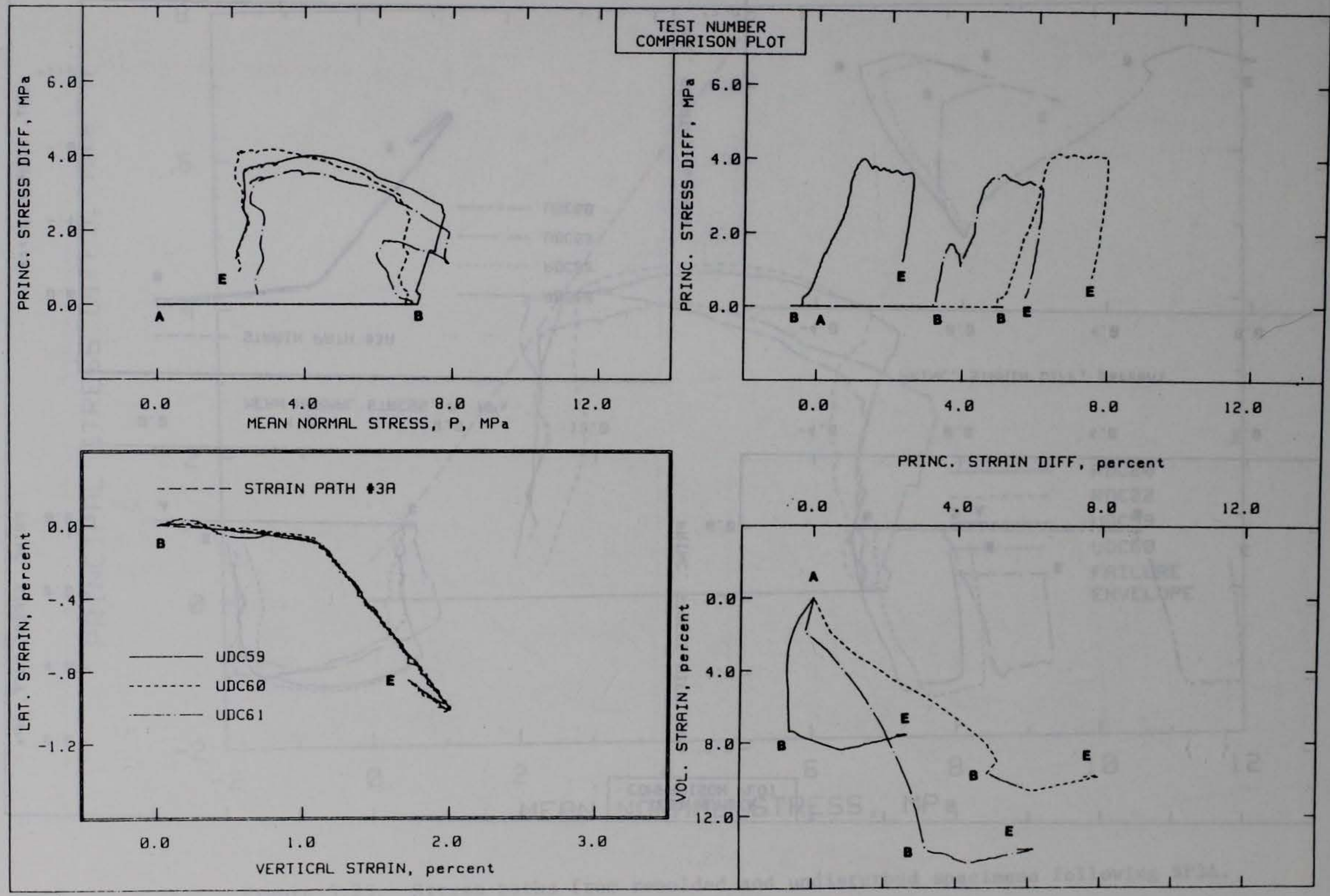

Figure 3.21. Strain path $3 \mathrm{~A}$ test results from undisturbed specimens. 


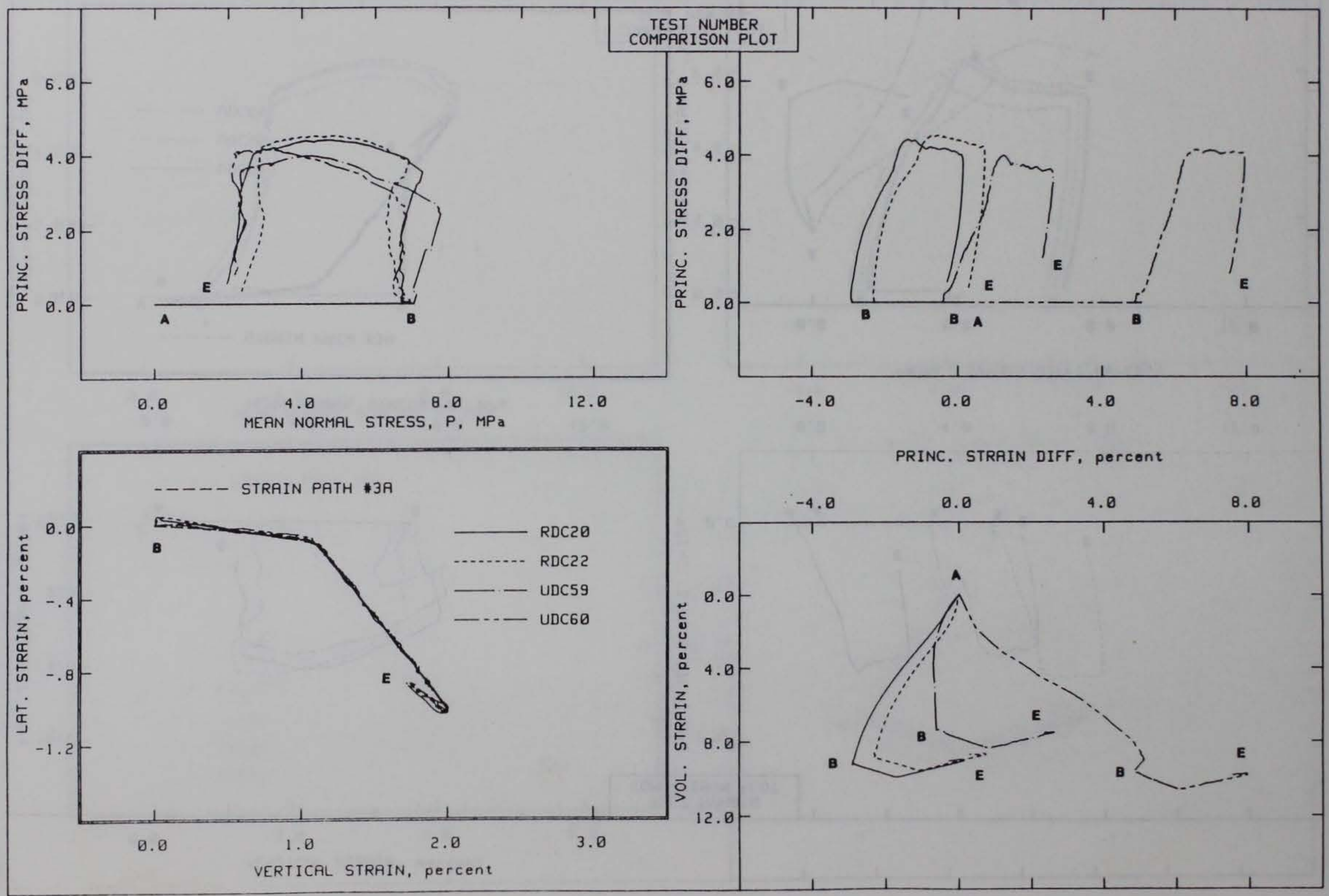

Figure 3.22. Comparison of strain path data from remolded and undisturbed specimens following SP3A. 


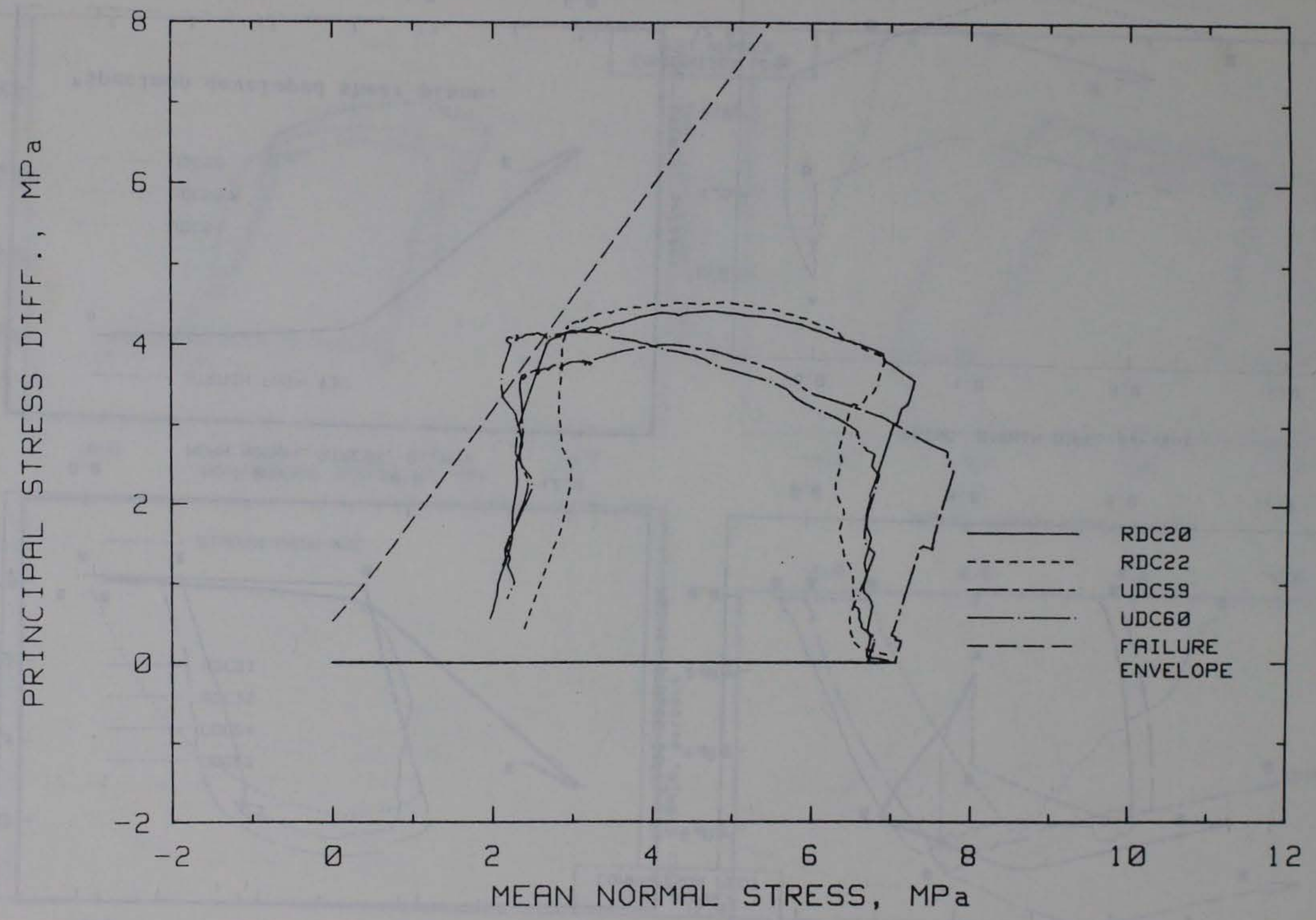

Figure 3.23. Stress paths from remolded and undisturbed specimens following SP3A: 

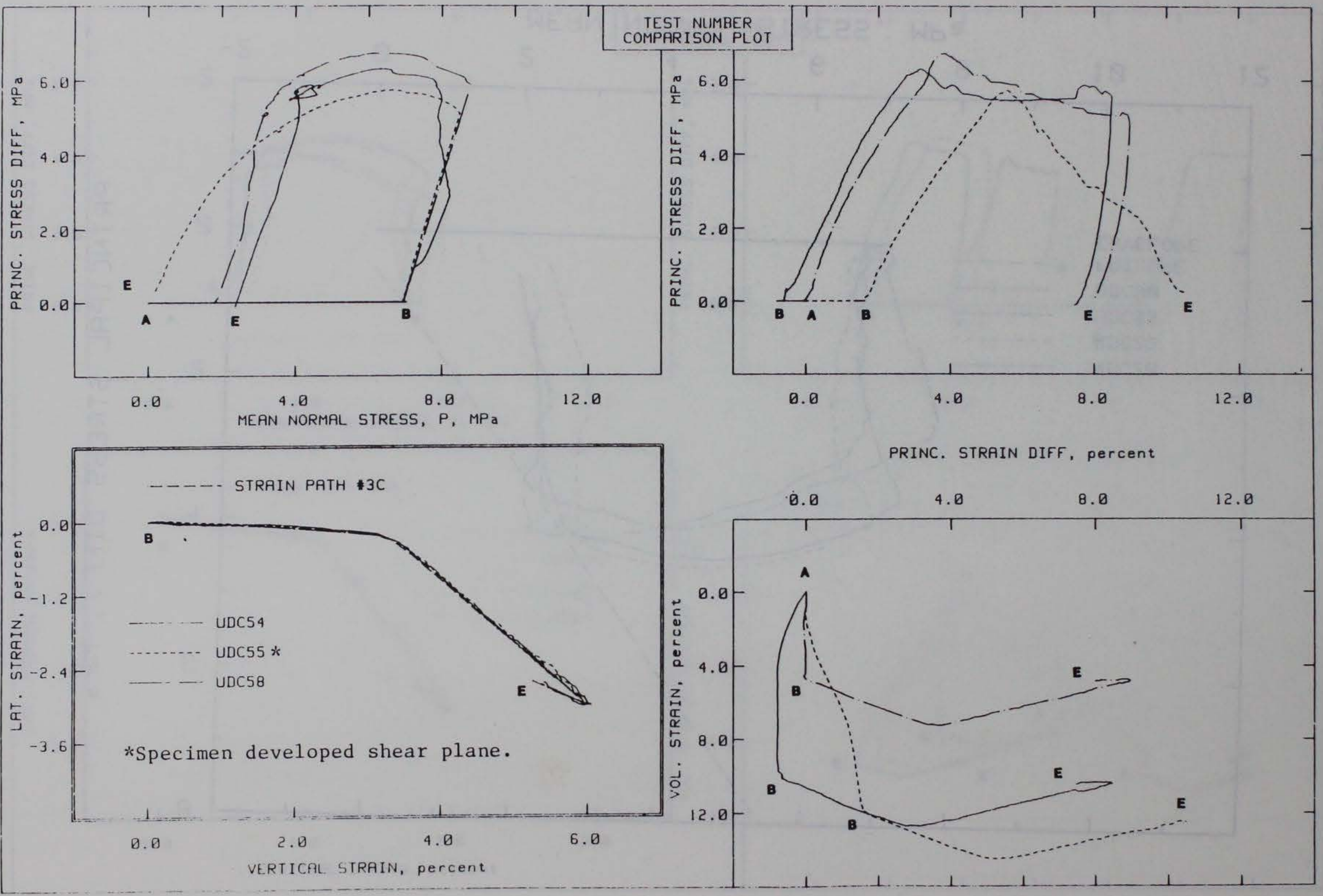

Figure 3.24. Strain path $3 \mathrm{C}$ test results from undisturbed specimens. 


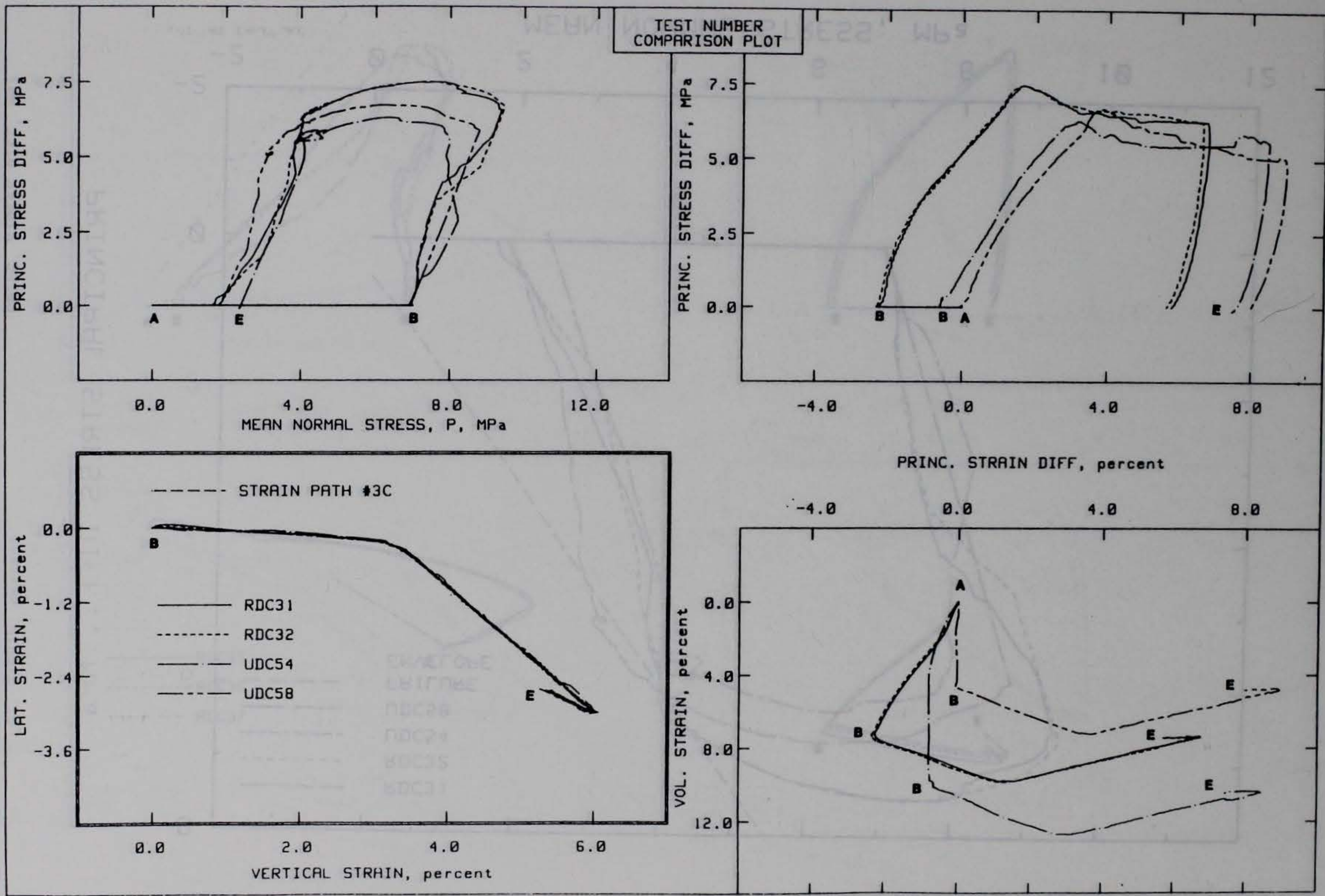

Figure 3.25. Comparison of strain path data from remolded and undisturbed specimens following SP3C. 


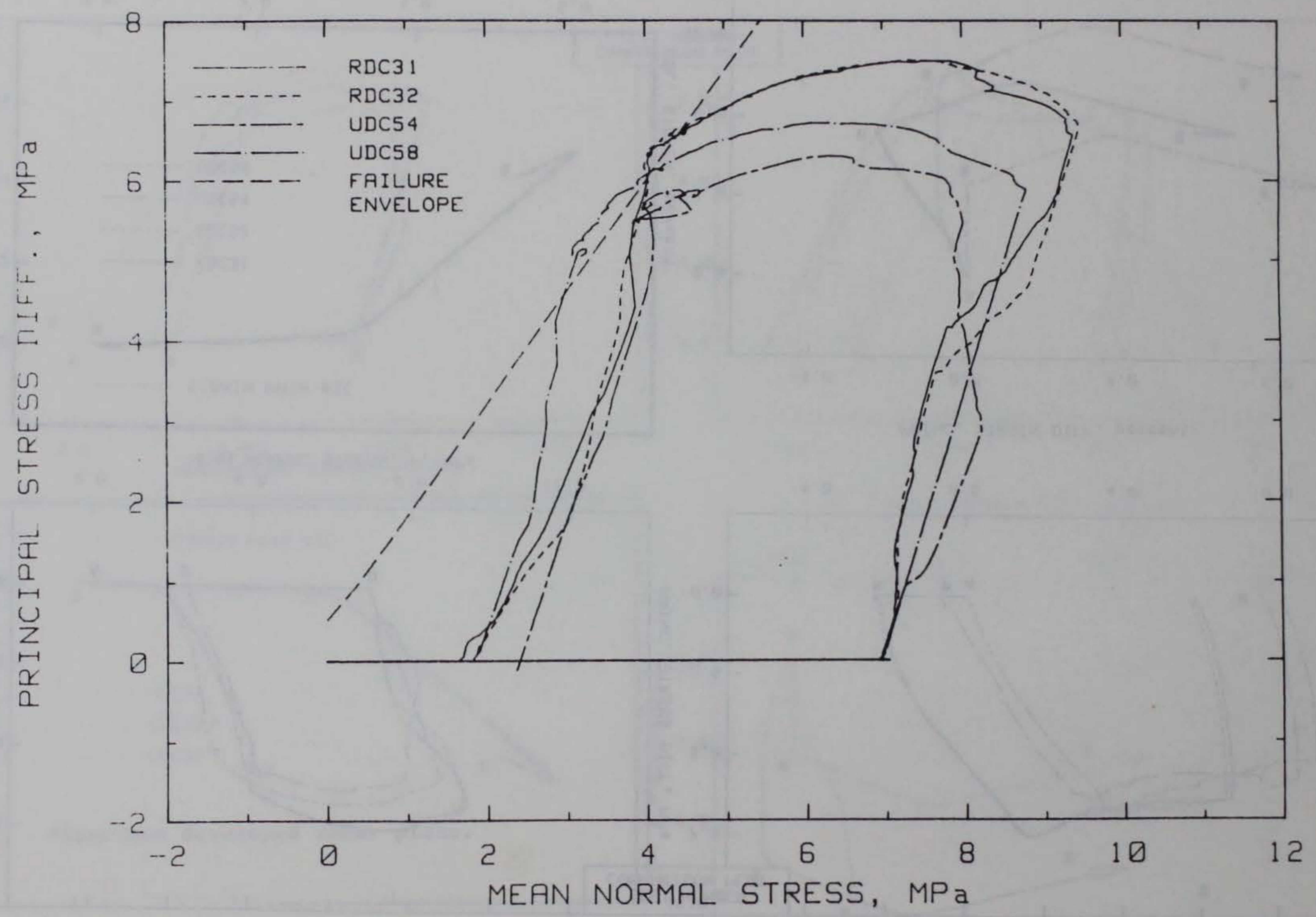

Figure 3.26. Stress paths from remolded and undisturbed specimens following SP3C. 


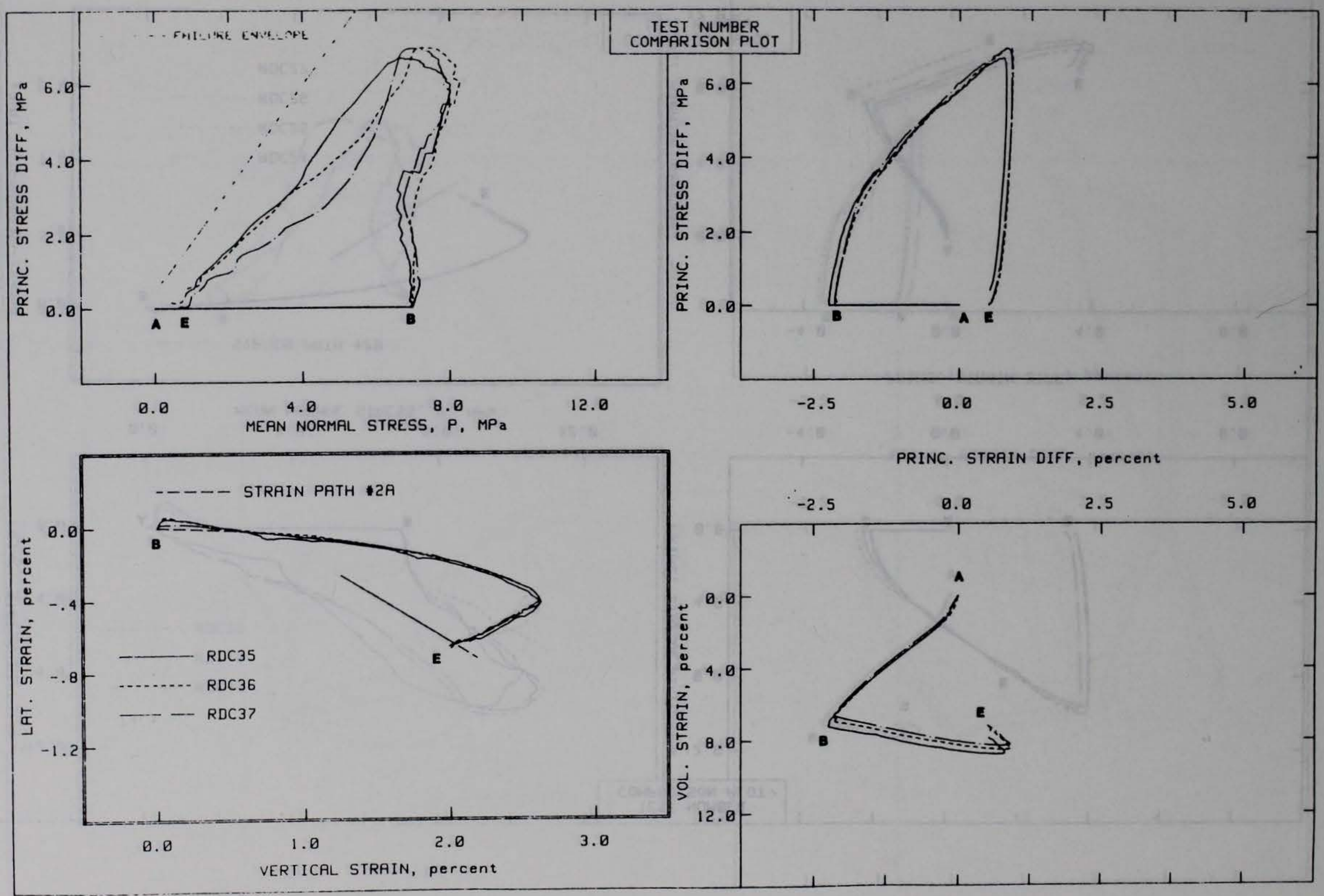

Figure 3.27. Strain path $2 \mathrm{~A}$ test results. 


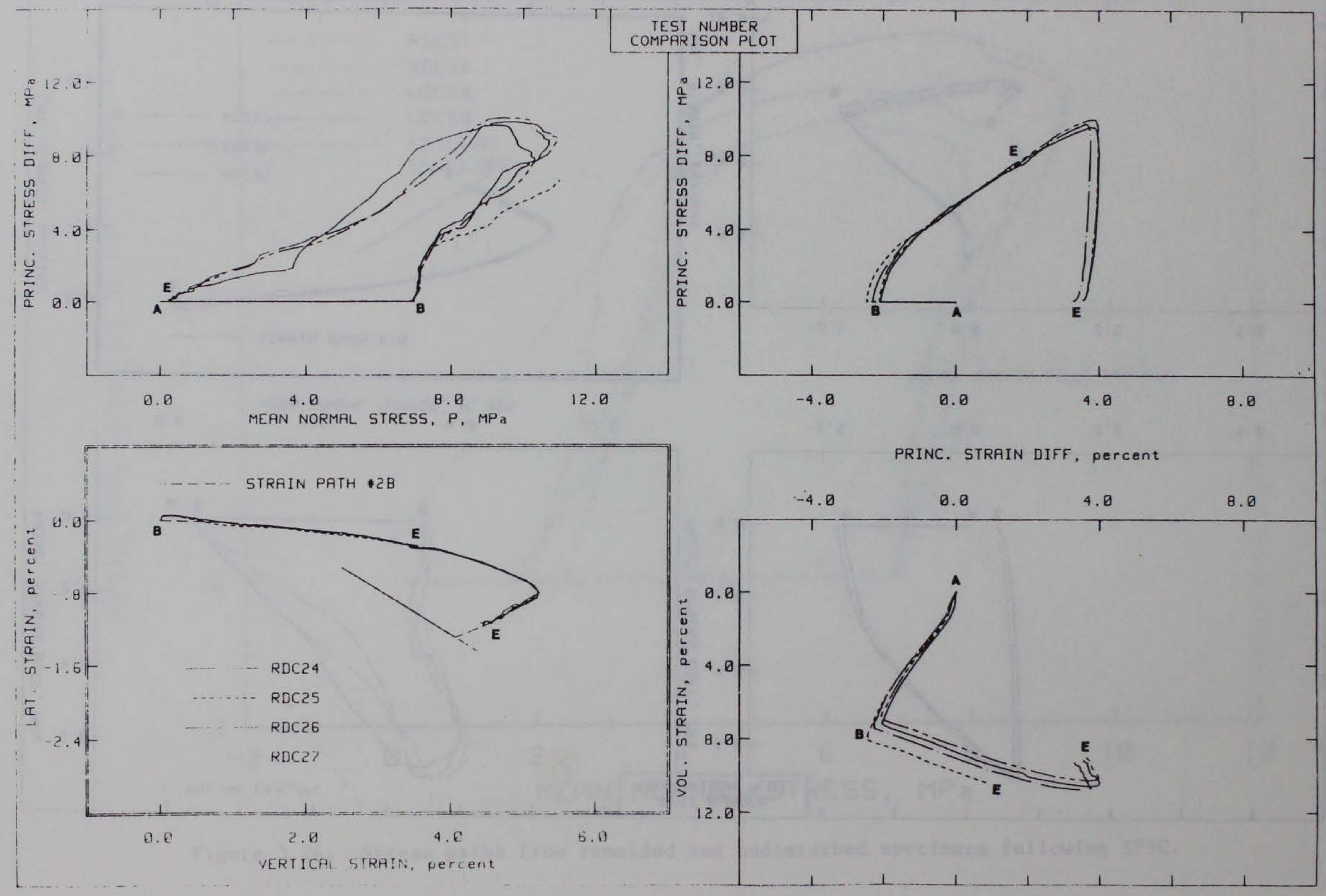

Figure 3.28. Strain path 2B test results. 


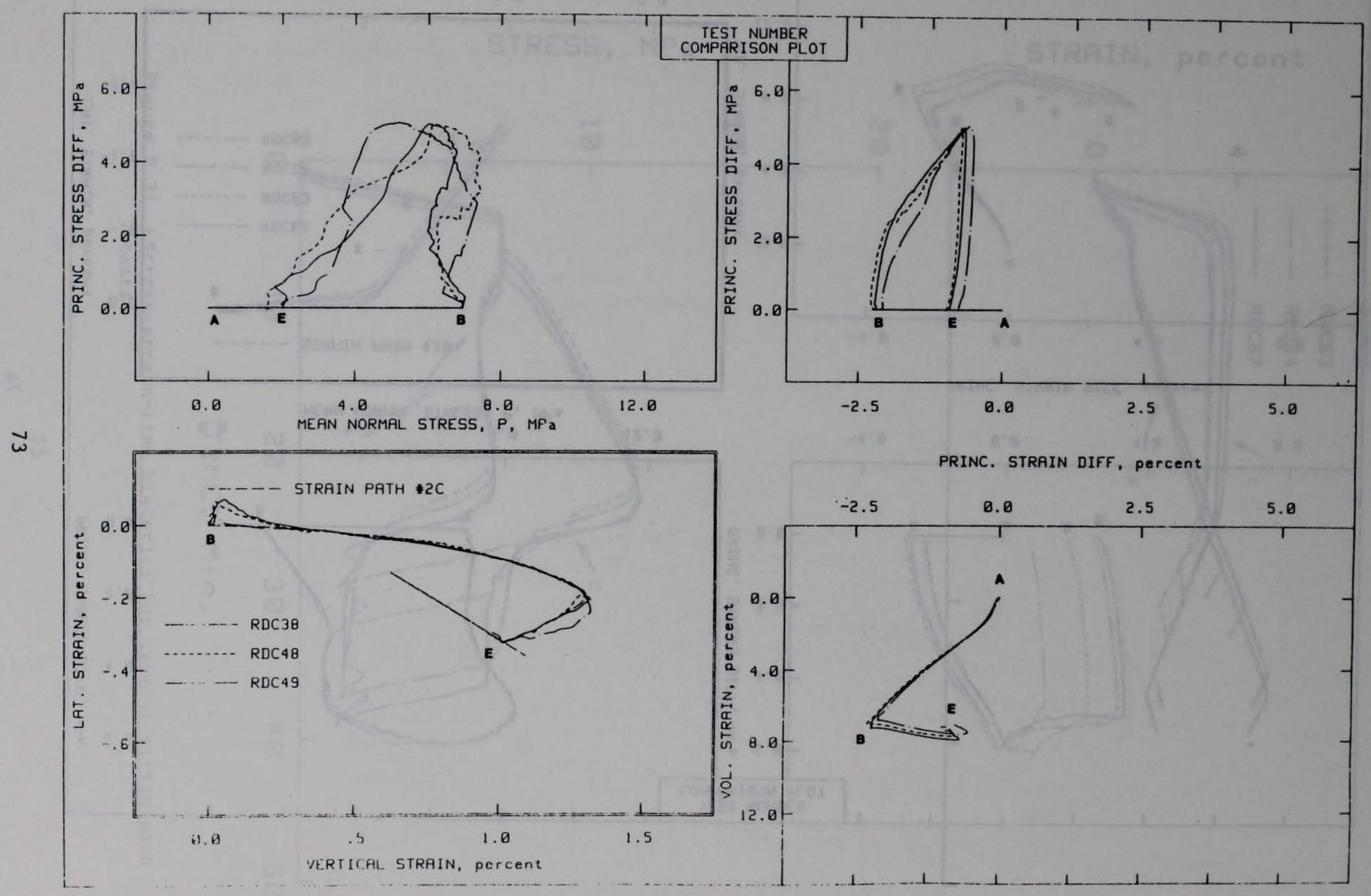

Figure 3.29. Strain path 2 C test results. 


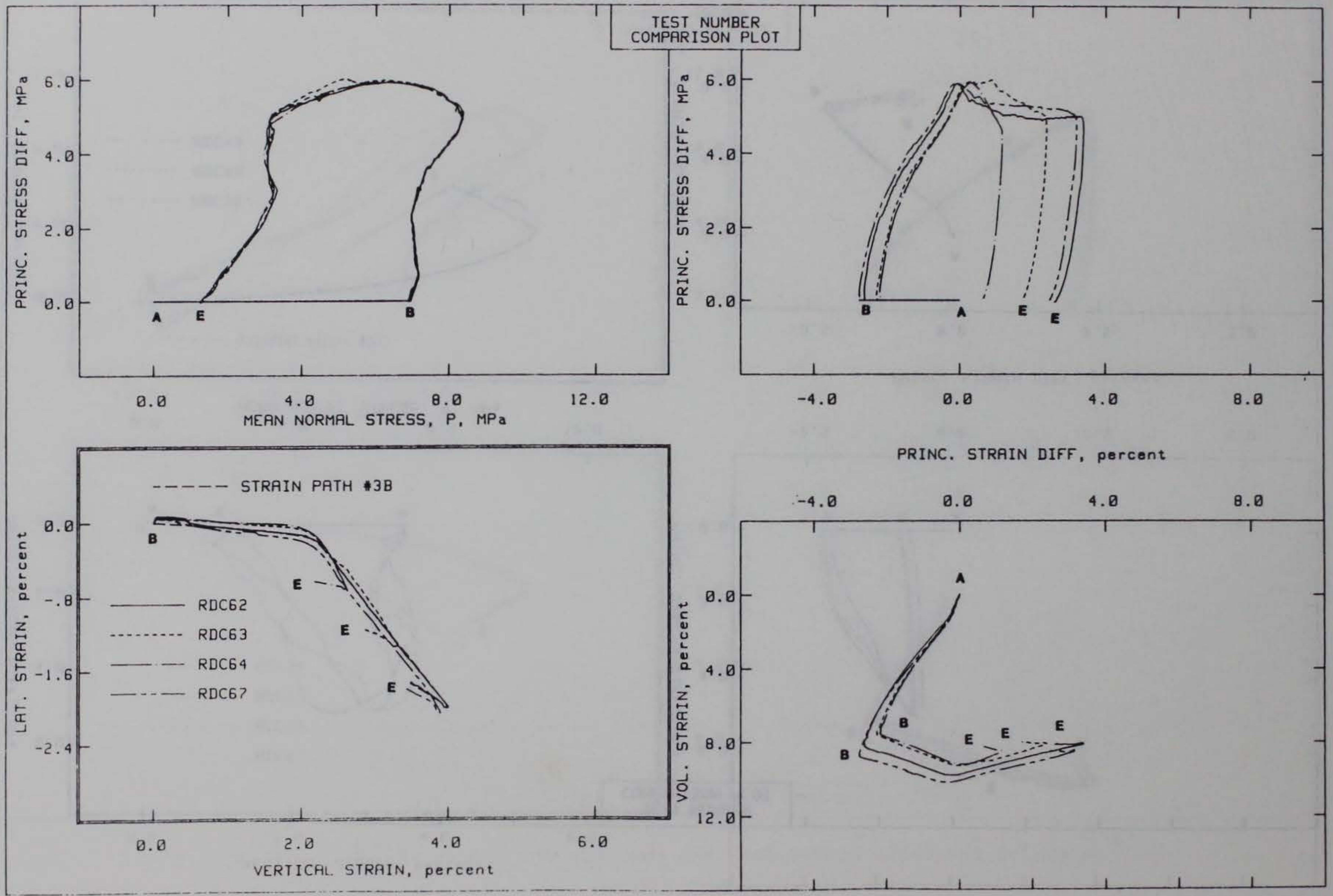

Figure 3.30. Reverse strain path test results. 

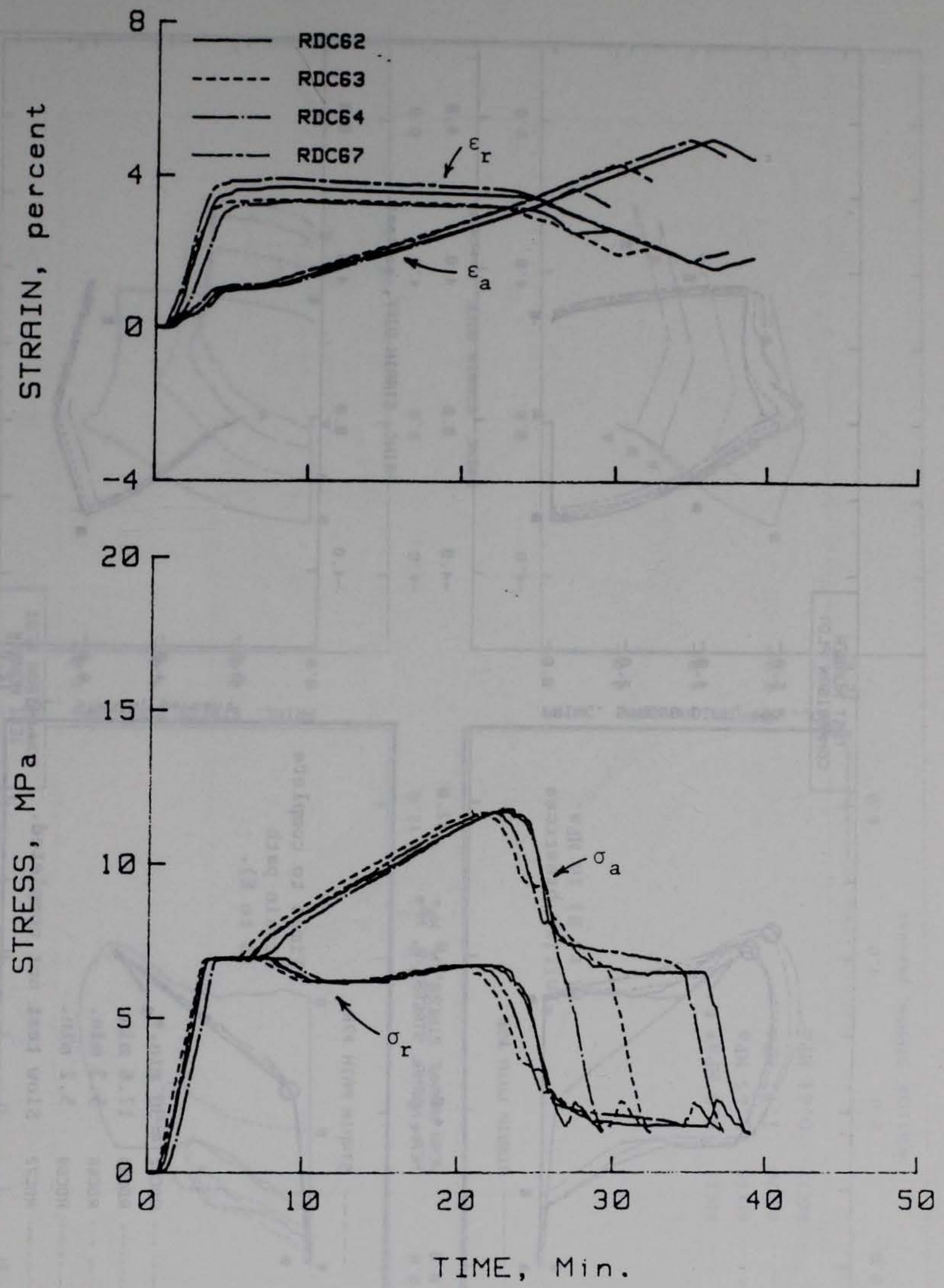

Figure 3.31. Stress-strain-time histories for reverse strain-path tests. 


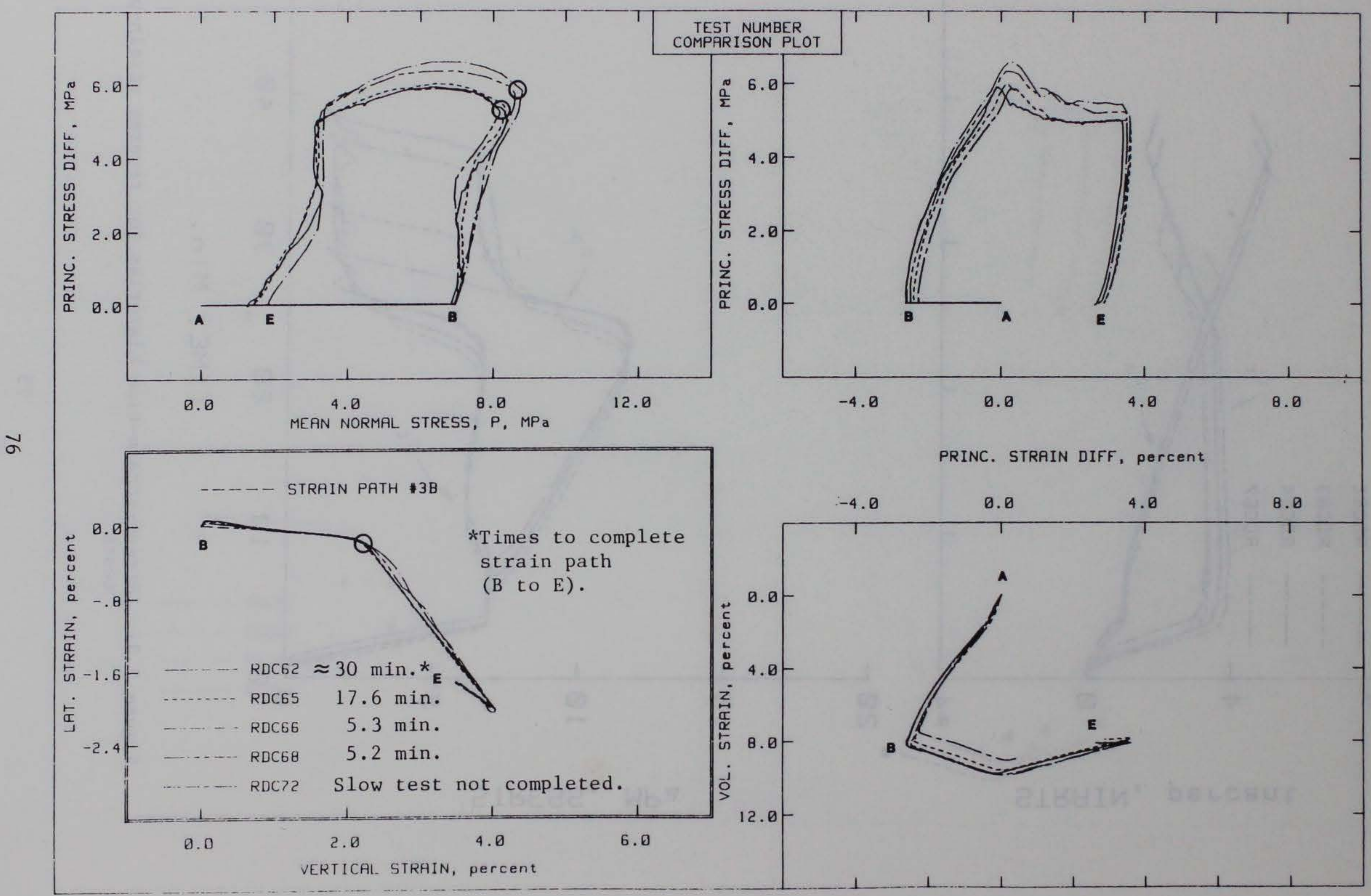

Figure 3.32. Strain-rate test results. 



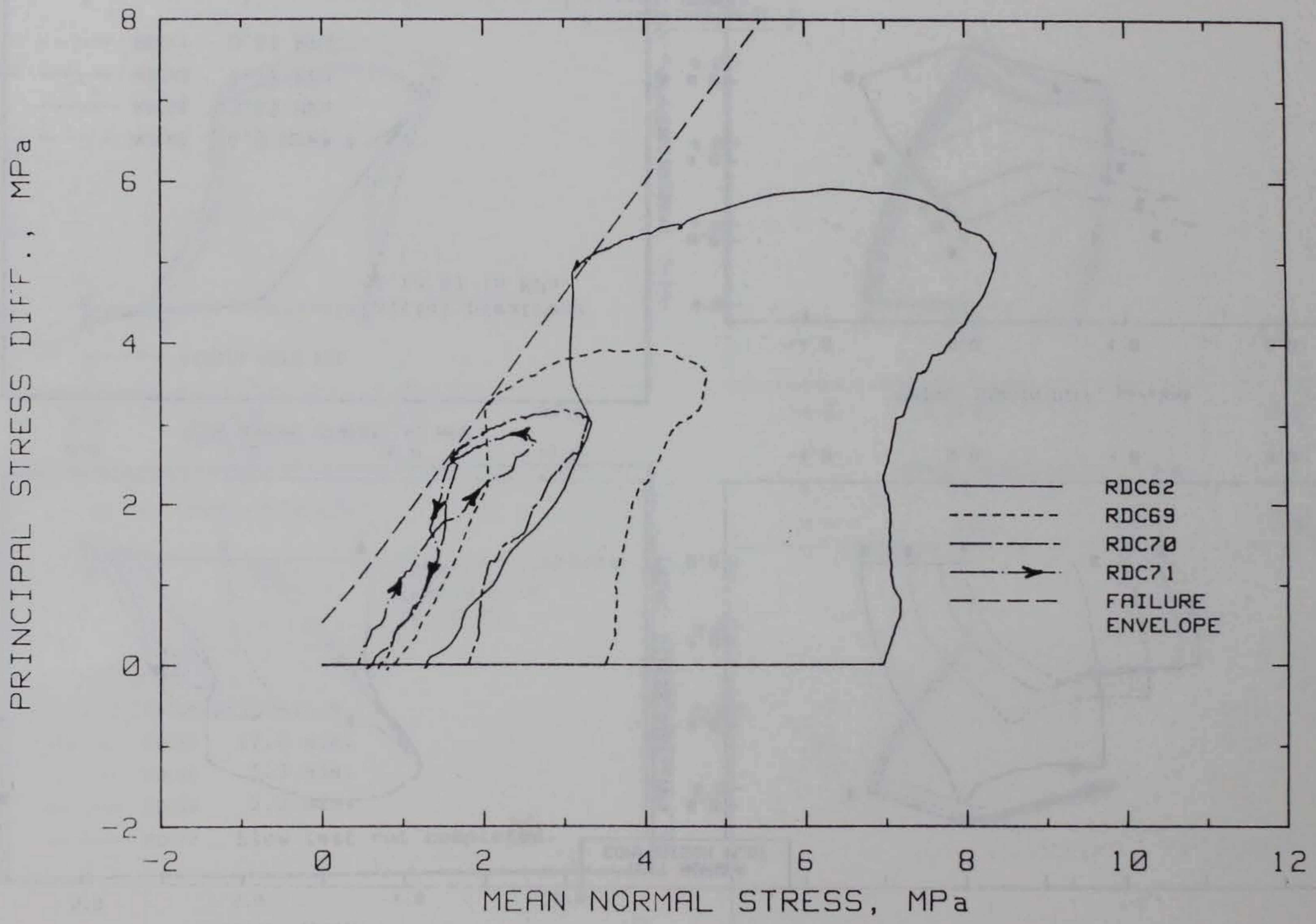

Figure 3.34. Stress paths from strain path tests at different levels of prestress. 


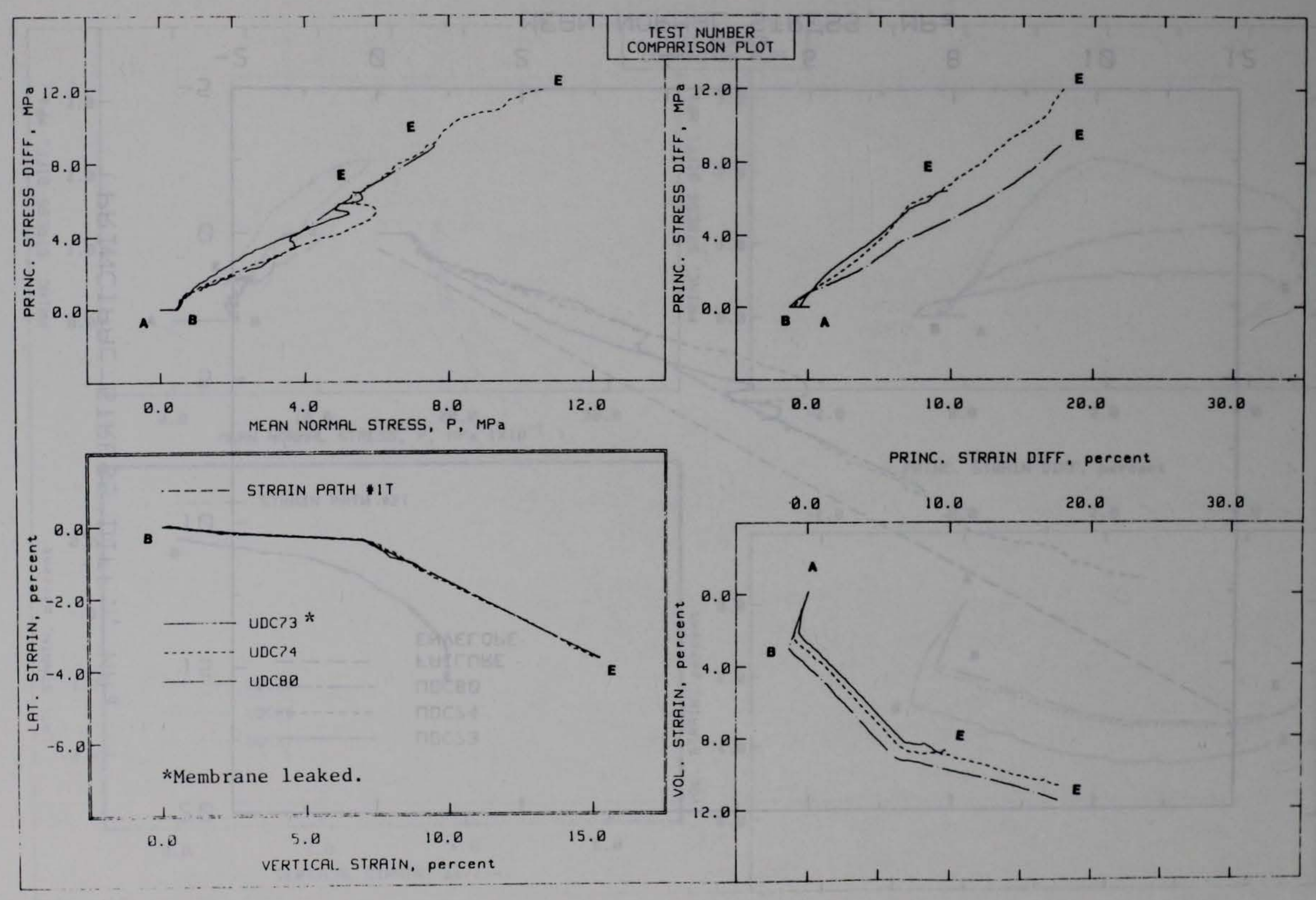

Figure 3.35. Strain path IT test results. 


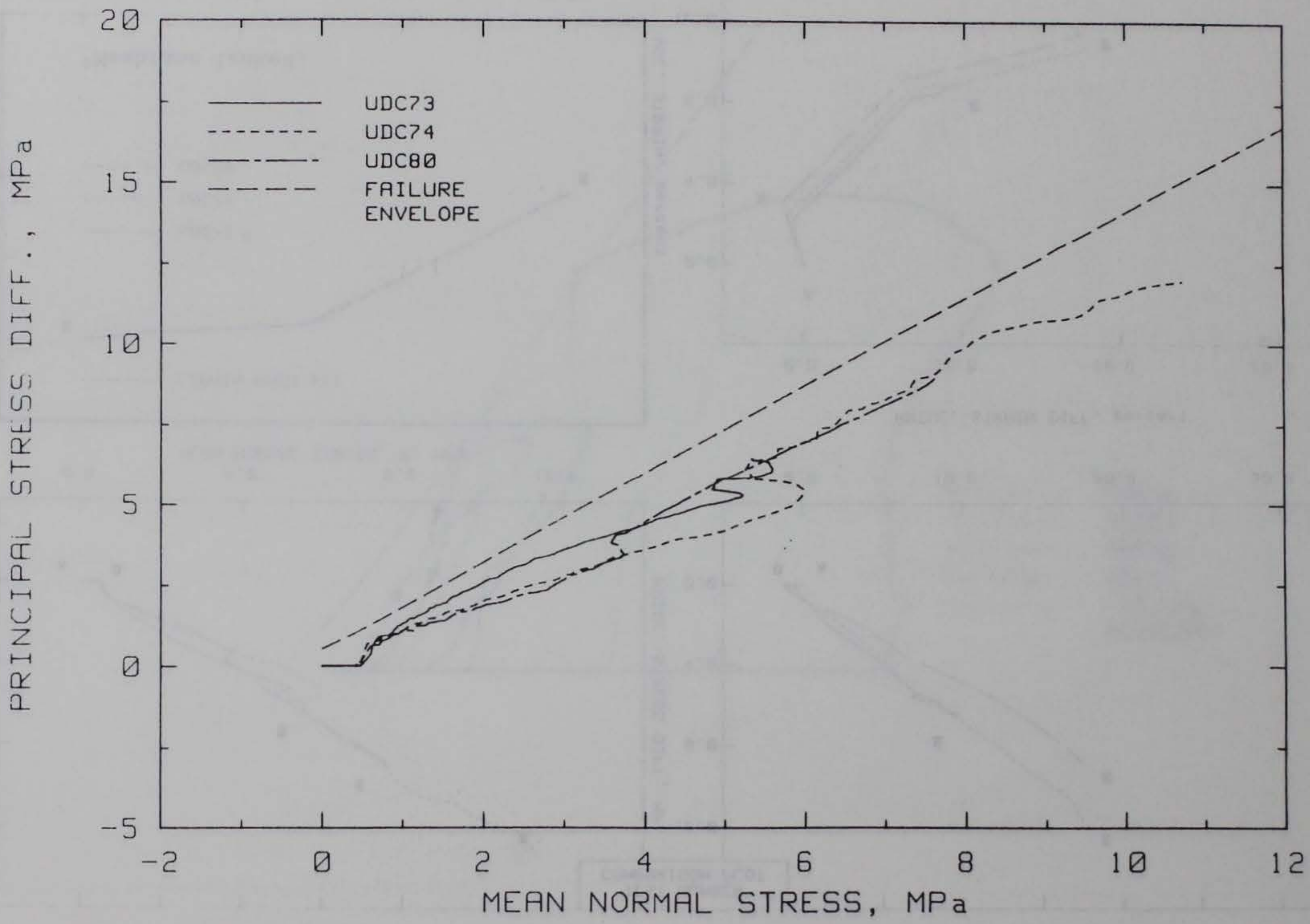

Figure 3.36. Stress paths from tests following SP1T. 

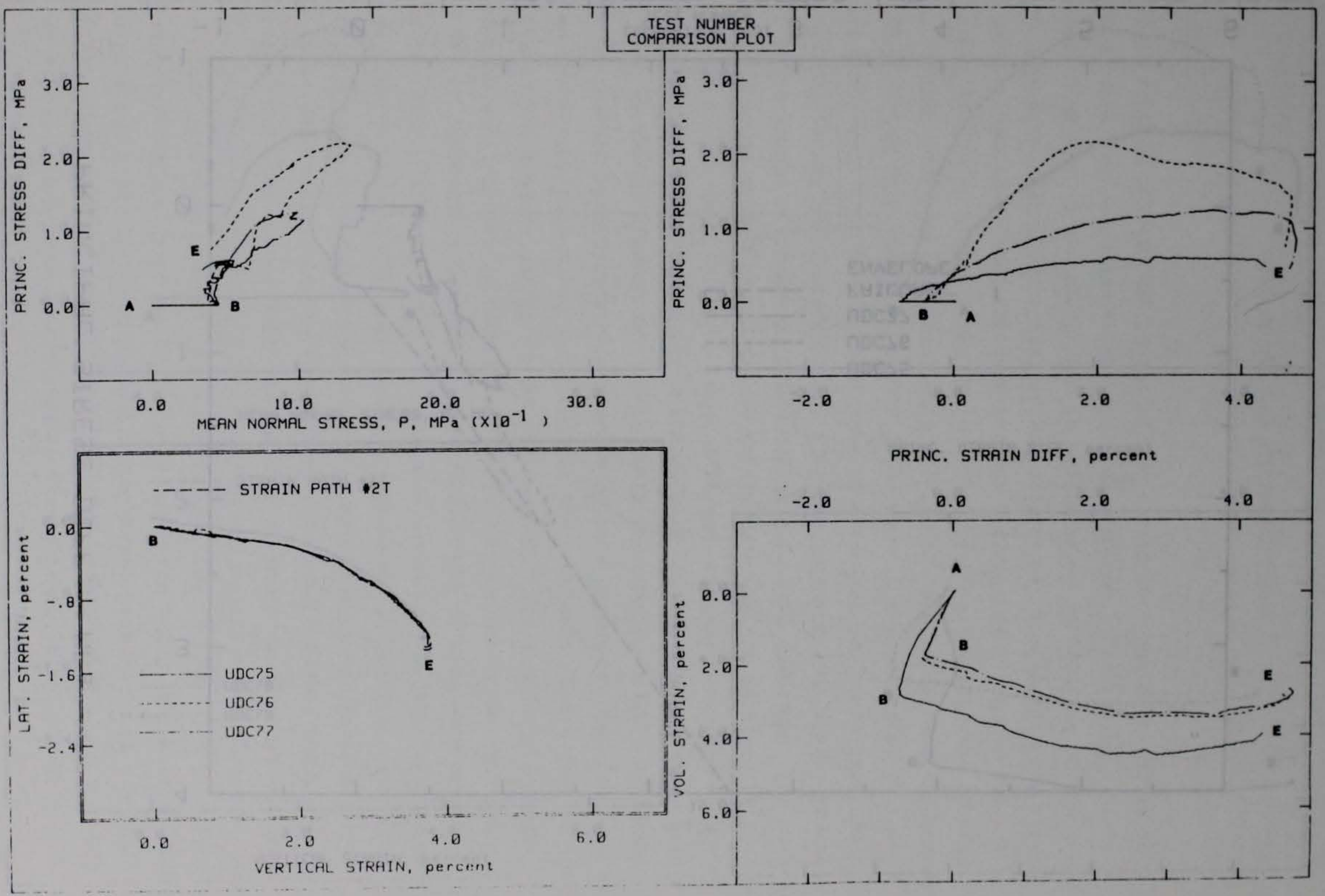

Figure 3.37 . Strain path $2 \mathrm{~T}$ test results at $0.41-\mathrm{MPa}$ prestress. 


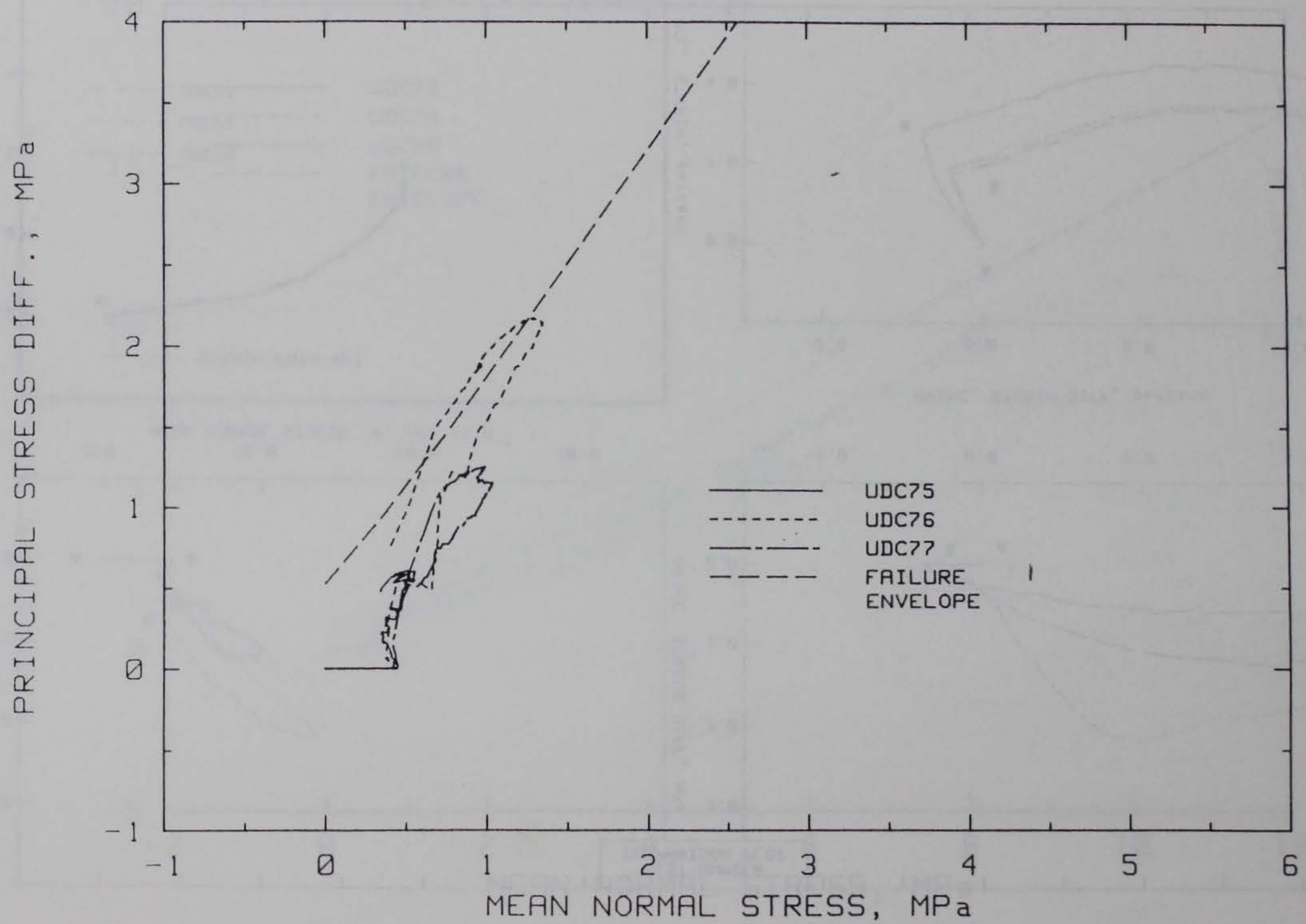

Figure 3.38. Stress paths from tests following SP2T at 0.41-MPa prestress. 


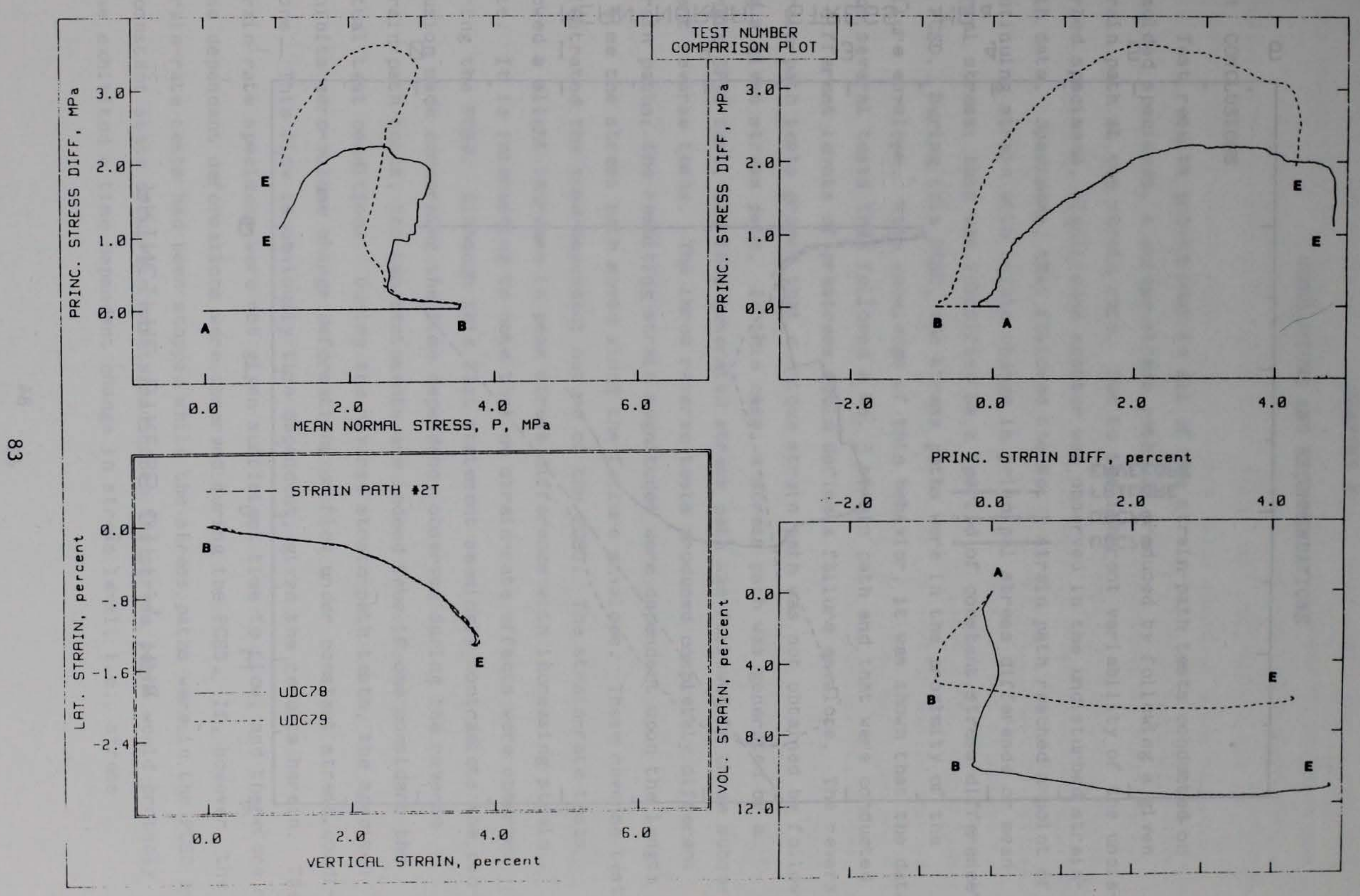

Figure 3.39. Strain path $2 \mathrm{~T}$ test results at $3.45-\mathrm{MPa}$ prestress. 


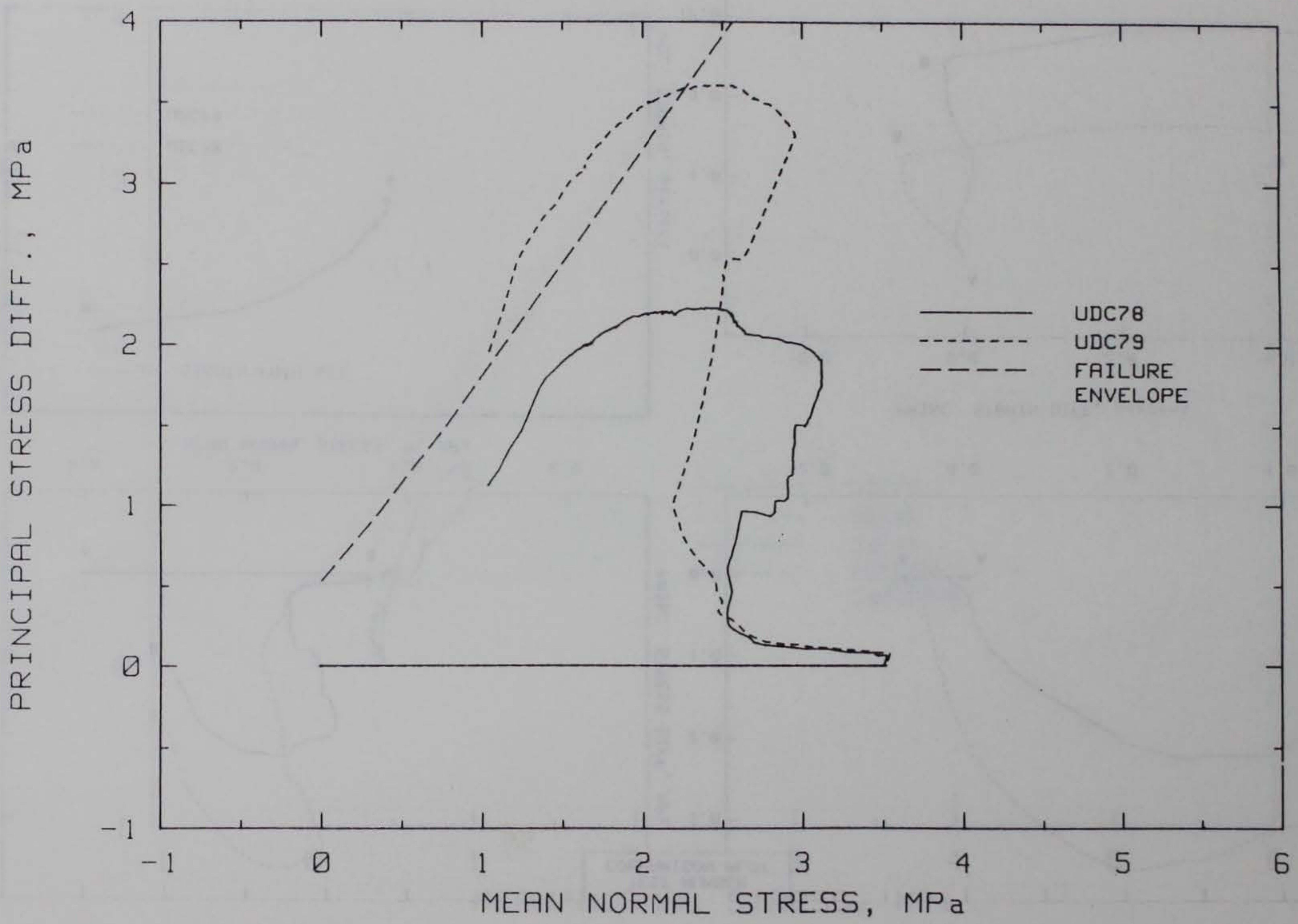

Figure 3.40. Stress paths from tests following SP2T at 3.45-MPa prestress. 


\section{CHAPTER 4 \\ CONCLUSIONS AND RECOMMENDATIONS}

\subsection{CONCLUSIONS}

Test results proved that in all of the strain-path tests conducted on remolded specimens, a unique stress path was produced by following a given strain path at one strain rate. Due to the inherent variability of the undisturbed specimens, significant scatter was observed in the undisturbed strainpath data. Specimens, that followed the No. 3 strain path reached a point of continuing strain with little change in principal stress difference or mean normal stress; this was identified as a "period of constant stress difference" or PCSD. During this PCSD, the stress paths were in the proximity of the failure envelope. With knowledge of this behavior, it was shown that the data from several tests that followed a No. 3 strain path and that were conducted at different levels of prestress would define a failure envelope. The reverse strain-path tests proved that a unique strain path was not obtained by following a given stress path. In this case, a stress path was generated by a single SP3B test, and this generated stress path was followed in three subsequent reverse tests. The three reverse tests produced completely different strain paths; the resulting strain magnitudes were dependent upon the length of time the stress path moved along the failure envelope. These reverse tests illustrated the time-dependent nature of the PCSD. The strain-rate tests showed a slight increase in peak stress difference with increasing strain rate. It is interesting to note that no strain-rate effects were observed during the PCSD. Although this last statement seemingly contradicts the conclusion made concerning the time dependency observed during the reverse strain-path tests, the two statements are indeed true if one considers the actual test conditions. During the reverse strain-path tests, the specimen exhibits zero-volume change deformations or flow under constant stress conditions. This flow is obviously time dependent, given the resuits herein. The strain-rate specimens were not given sufficient time to flow, and therefore no time dependent deformations were observed during the PCSD. If, however, the strain-rate tests had been stopped while the stress paths were in the PCSD and a constant state of strain maintained, then the stress paths would probably have exhibited a time dependent change in stress level, i.e., stress 
relaxation would have been observed. Thus, time dependent deformations were not observed in the strain-rate tests because the appropriate stress and strain conditions were not applied.

In the stress-path test phase, both compression and extension tests were conducted under constant axial and constant radial stress boundary conditions with a manually controlled pressure system. The available test data were insufficient to characterize the hardening response of the CARES material as either isotropic or kinematic. Several load-unload-reload tests were successfully conducted on the CARES material; these tests exhibited a type of "stiffening" response. The specimens that were deformed to larger values of strain in extension and reloaded into compression achieved higher val ues of stress difference. This behavior was not observed when the specimens were unloaded to zero stress difference, then reloaded; in those cases, the stress-strain curve would bend over upon reaching the point of initial unloading. The information gained from these tests could be quite useful for constitutive model fitting.

\subsection{RECOMMENDATIONS}

This report documents the second of two laboratory test programs which investigated the responses of two dry granular soils to strain-path loadings; in each investigation, similar soil behavior was measured. In terms of pure research, it would be interesting to further investigate the response of a given soil while it is being loaded into the PCSD. The behavior of specimens during this phase of the strain-path tests appears to be very complicated, yet it seems to be yielding some interesting data on the response of soils in a quasi-unstable state. Rather than using a strain-controlled loading system, a stress-controlled loading system may provide more information about the "stability" of specimens in the proximity of the failure envelope. Another improvement that would provide valuable data is the measurement of accurate vol umetric strains. These data are especially needed during the PCSD.

Two new strain paths were introduced in this report, strain paths $1 \mathrm{~T}$ and $2 \mathrm{~T}$; these paths were only conducted on undisturbed test specimens. A more representative data base could be collected if these paths were conducted on remolded specimens. One must again state that subtle changes in response will not be found when testing undisturbed specimens; the variability is too great. 


\section{REFERENCES}

1. S. A. Akers; "Axisymmetric Strain Path Tests on Nellis Baseline Sand"; October 1983; US Army Engineer Waterways Experiment Station, Vicksburg, MS.

2. S. A. Akers; "Axisymmetric Strain Path Tests on Undisturbed Specimens from the DIRECT COURSE Site"; January 1984; US Army Engineer Waterways Experiment Station, Vicksburg, MS.

3. H. Y. Ko and R. W. Meier; "Cubical Test Data and Strain Path Testing on Nellis Baseline Sand"; January 1983; Department of Civil, Environmental and Architectural Engineering, University of Colorado, Boulder, CO; prepared for the US Army Engineer Waterways Experiment Station, Vicksburg, MS.

4. P. V. Lade; "Strain-Path Tests on Yuma Soil"; October 1983; prepared by the University of California at Los Angeles, Los Angeles, CA; prepared for Applied Theory, Inc., Los Angeles, CA.

5. US Army Engineer Waterways Experiment Station; "The Unified Soil Classification System"; Technical Memorandum No. 3-357, April 1960 (reprinted May 1967, Amended 1 May 1980), Vicksburg, MS.

6. J. D. Cargile and R.E. Wahl; "Geotechnical Investigation for the CARES-Dry Site: Results from Subsurface Exploration Programs"; October 1983; US Army Engineer Waterways Experiment Station, Vicksburg, MS..

7. S. A. Akers and P. A. Reed; "WES High-Pressure Uniaxial Strain and Triaxial Shear Test Equipment"; Miscellaneous Paper (in publication); US Army Engineer Waterways Experiment Station, Vicksburg, MS.

8. J. D. Cargile; "Geotechnical Investigation for the CARES-Dry Site: Laboratory Test Results"; February 1984; US Army Engineer Waterways Experiment Station, Vicksburg, MS. 
HYDROSTATIC COMPRESSION TEST RESULTS 


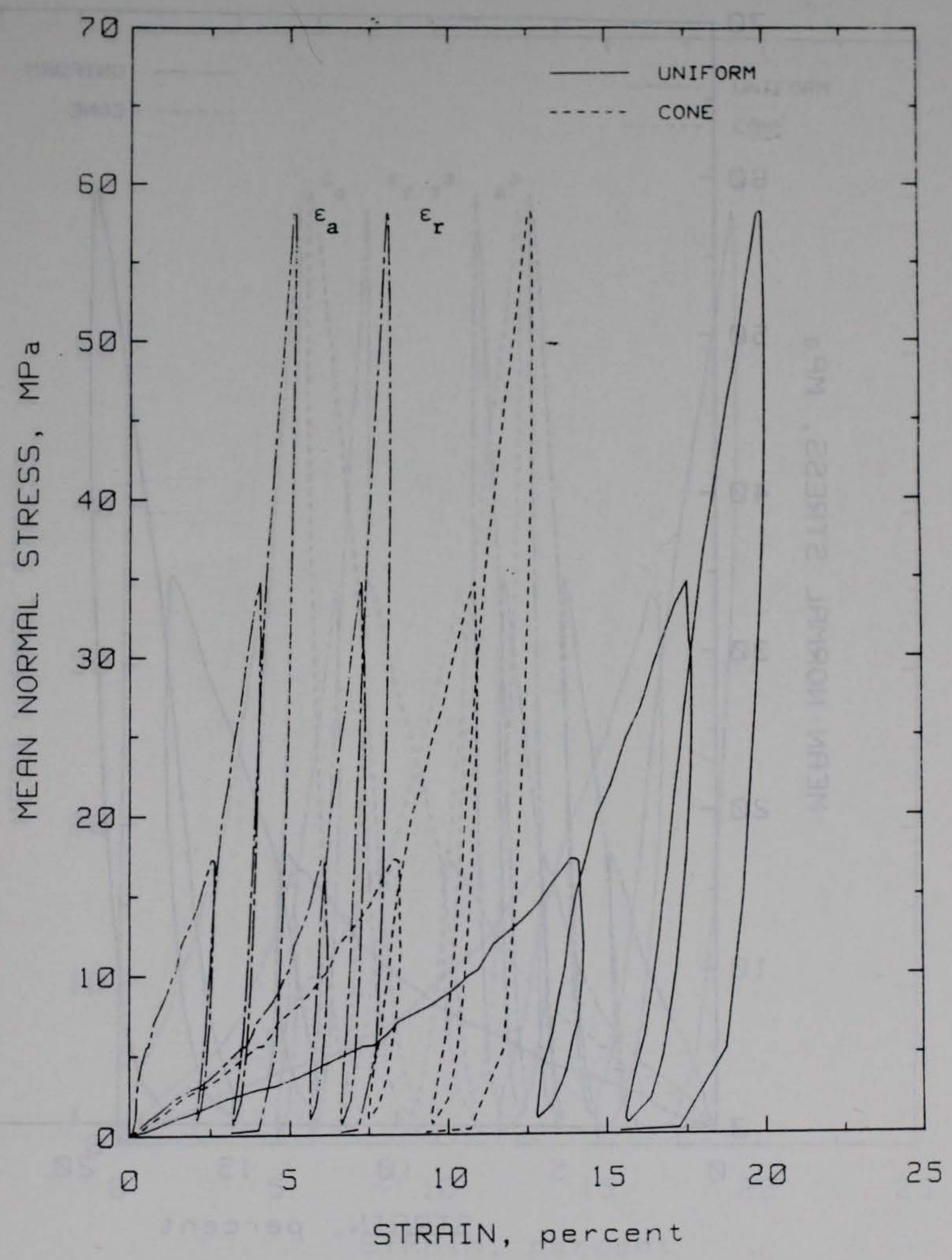

TEST NO: RDCOI 


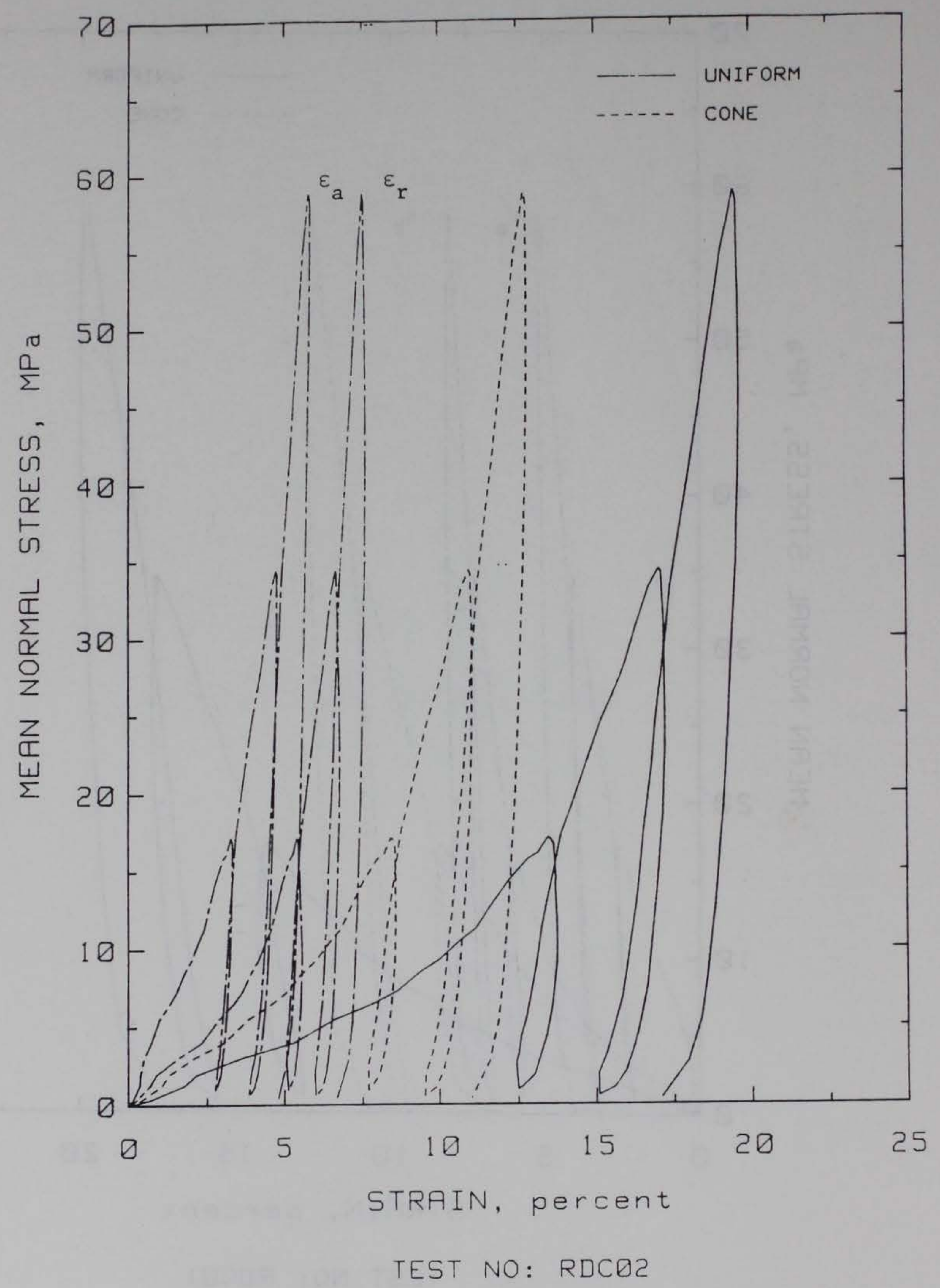

PLATE 2 


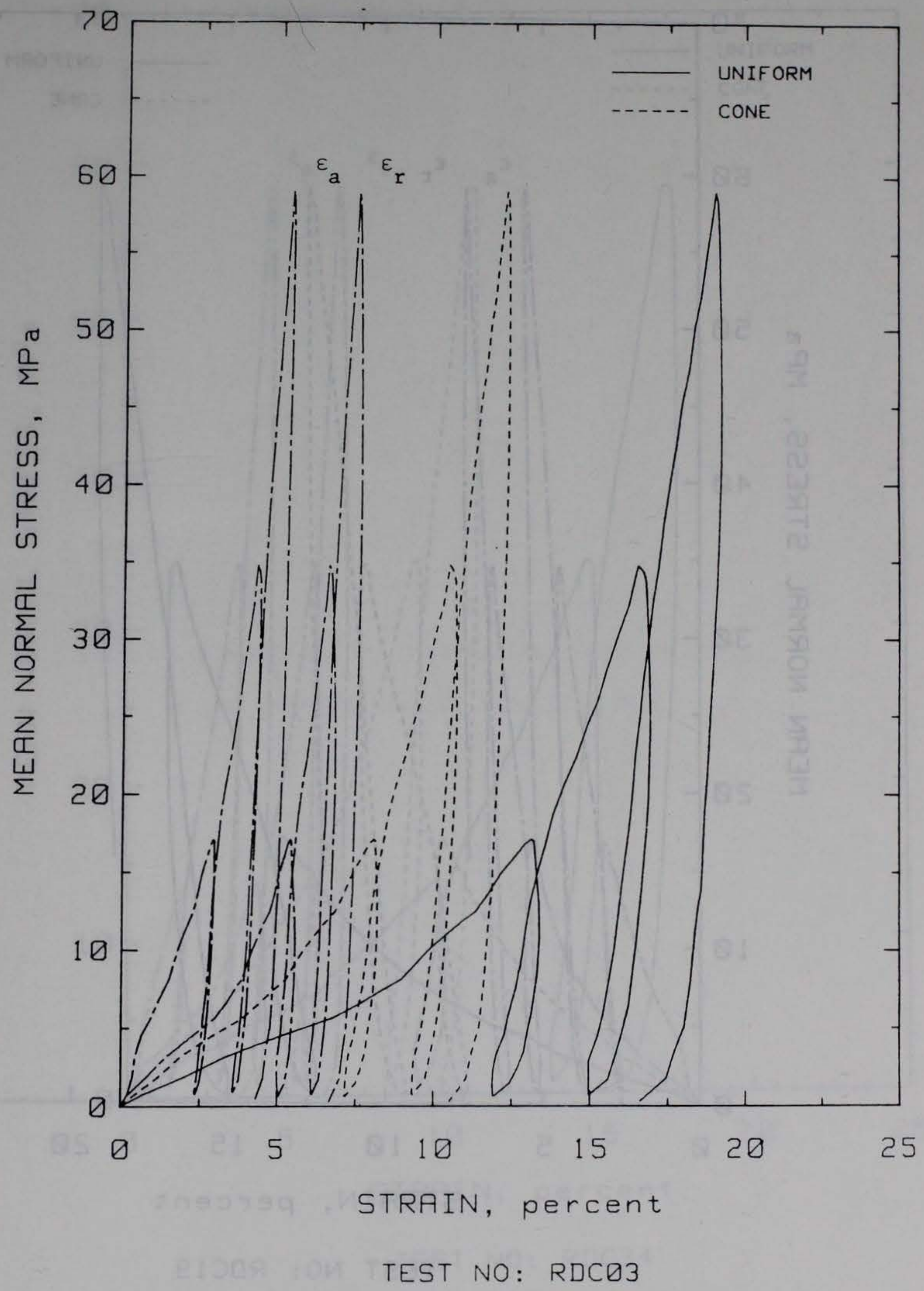

PLATE 3 


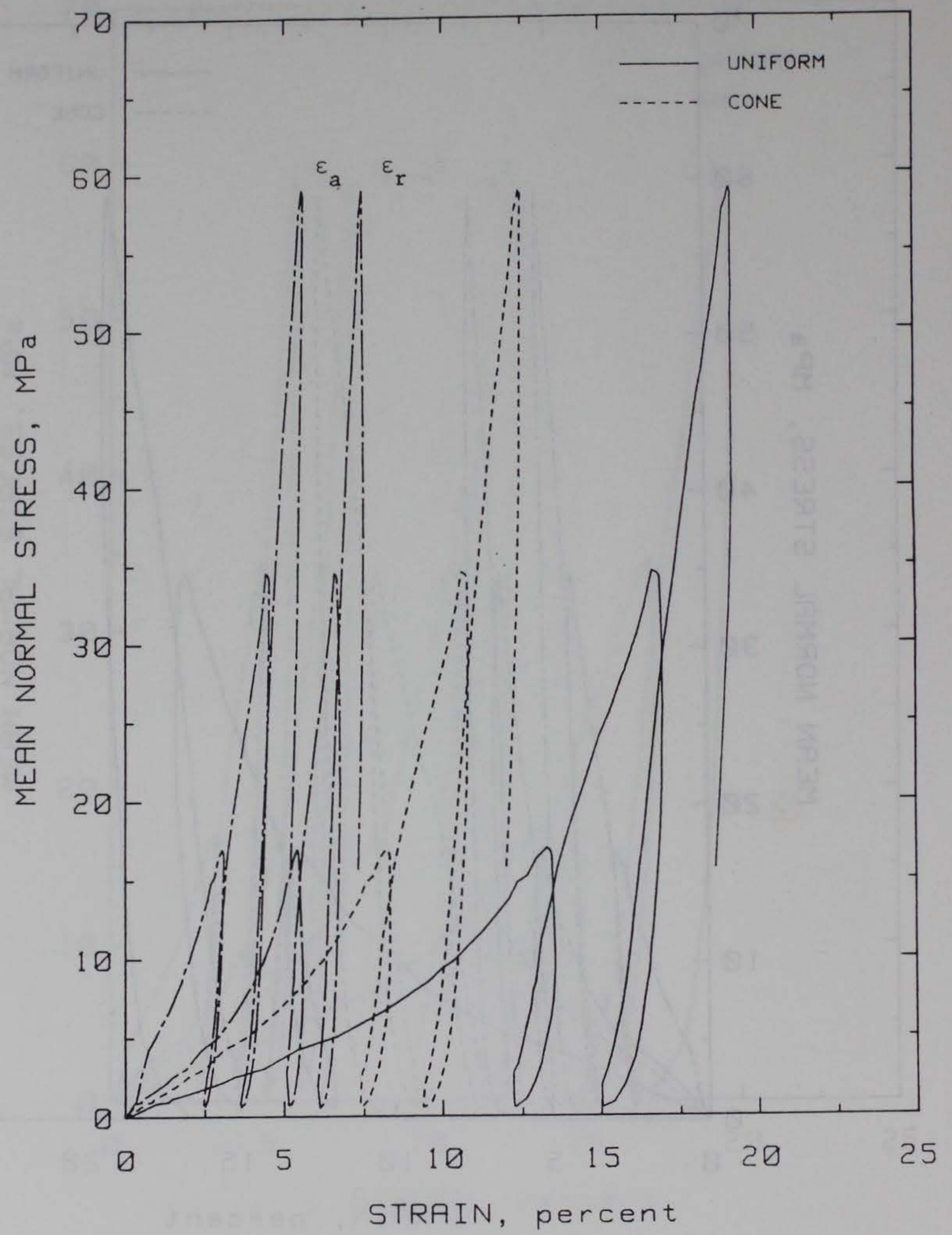

TEST NO: RDC19 


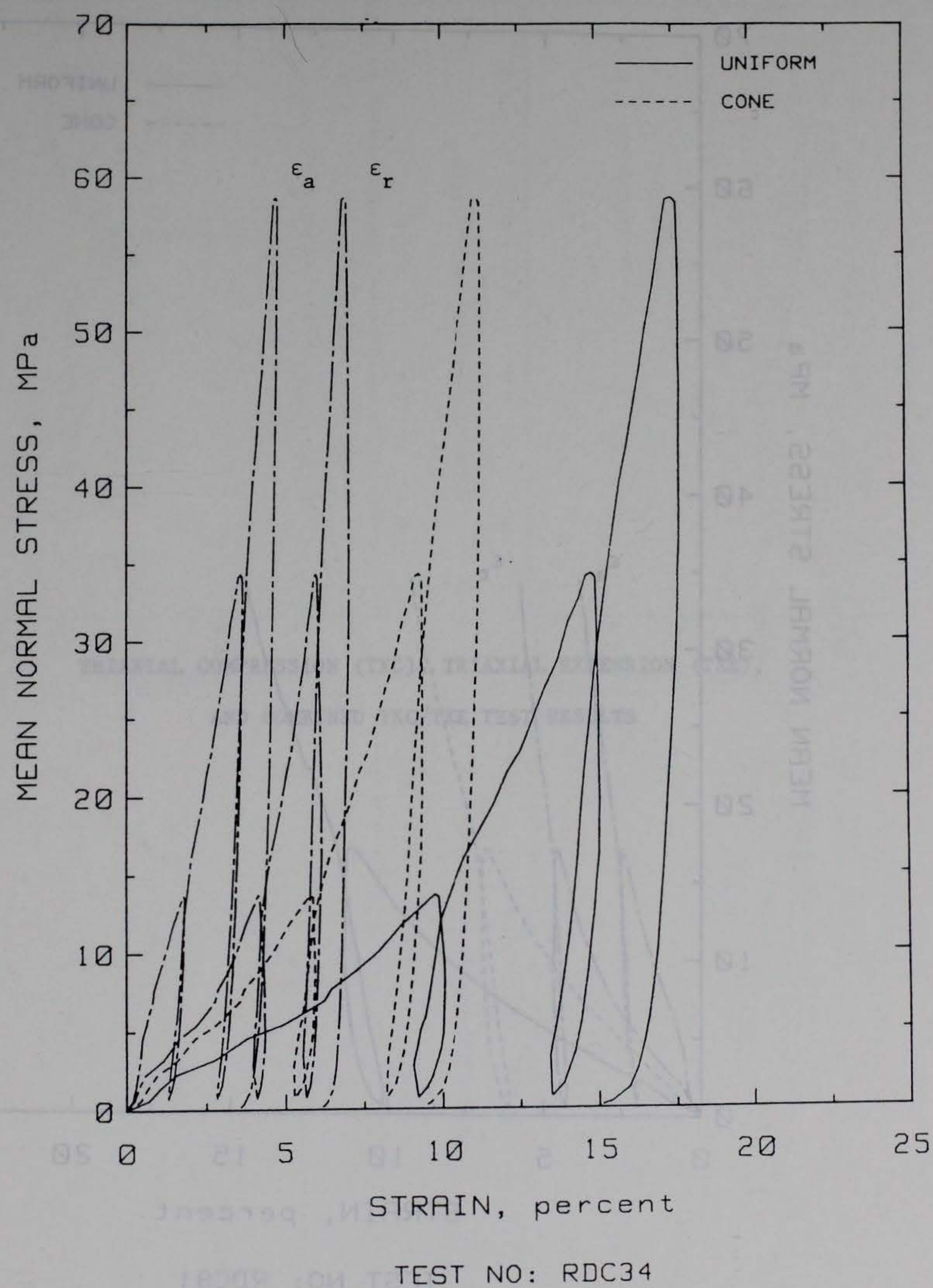




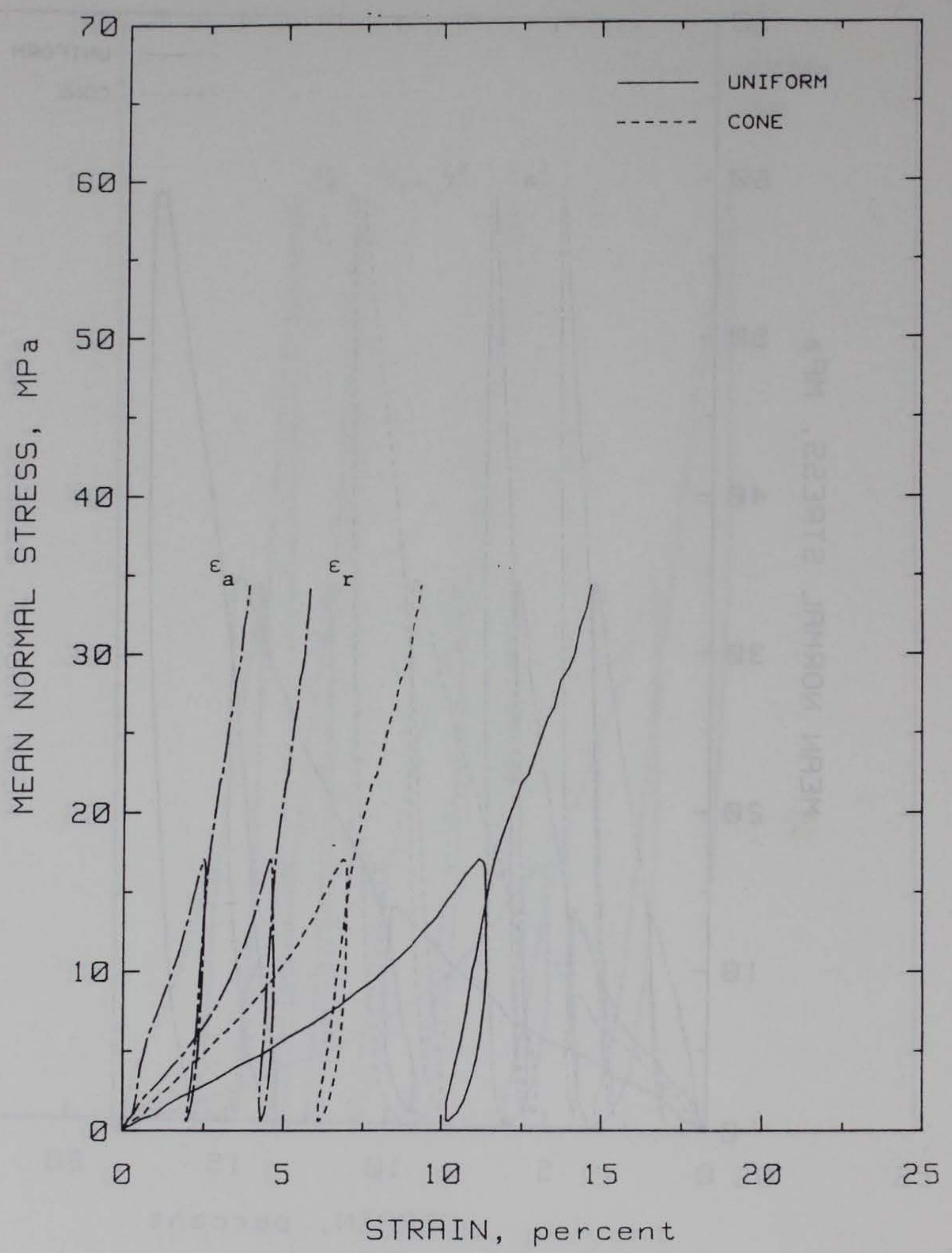

TEST NO: RDC8 1 
TRIAXIAL COMPRESSION (TXC), TRIAXIAL EXTENSION (TXE), AND COMBINED TXC/TXE TEST RESULTS 


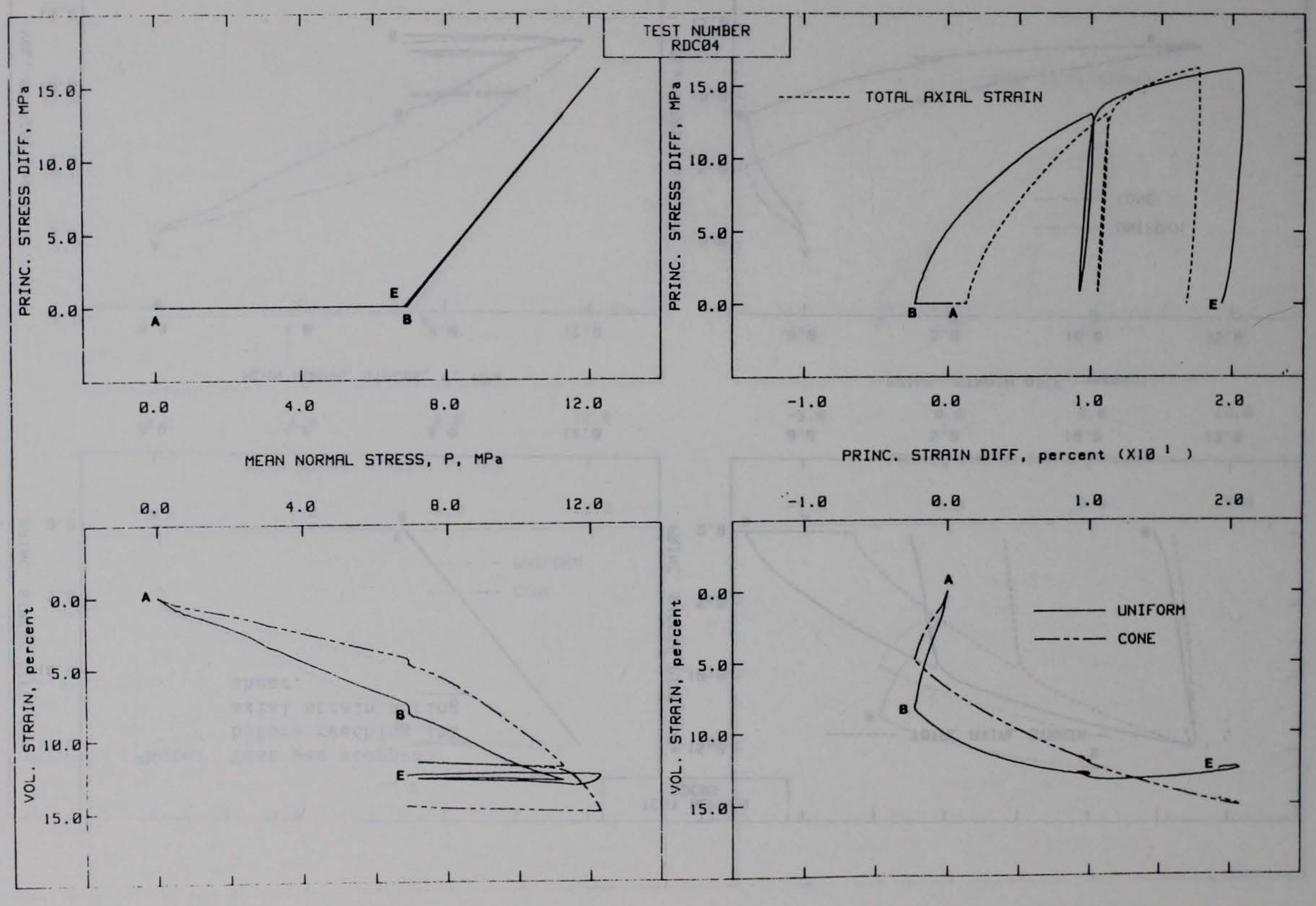




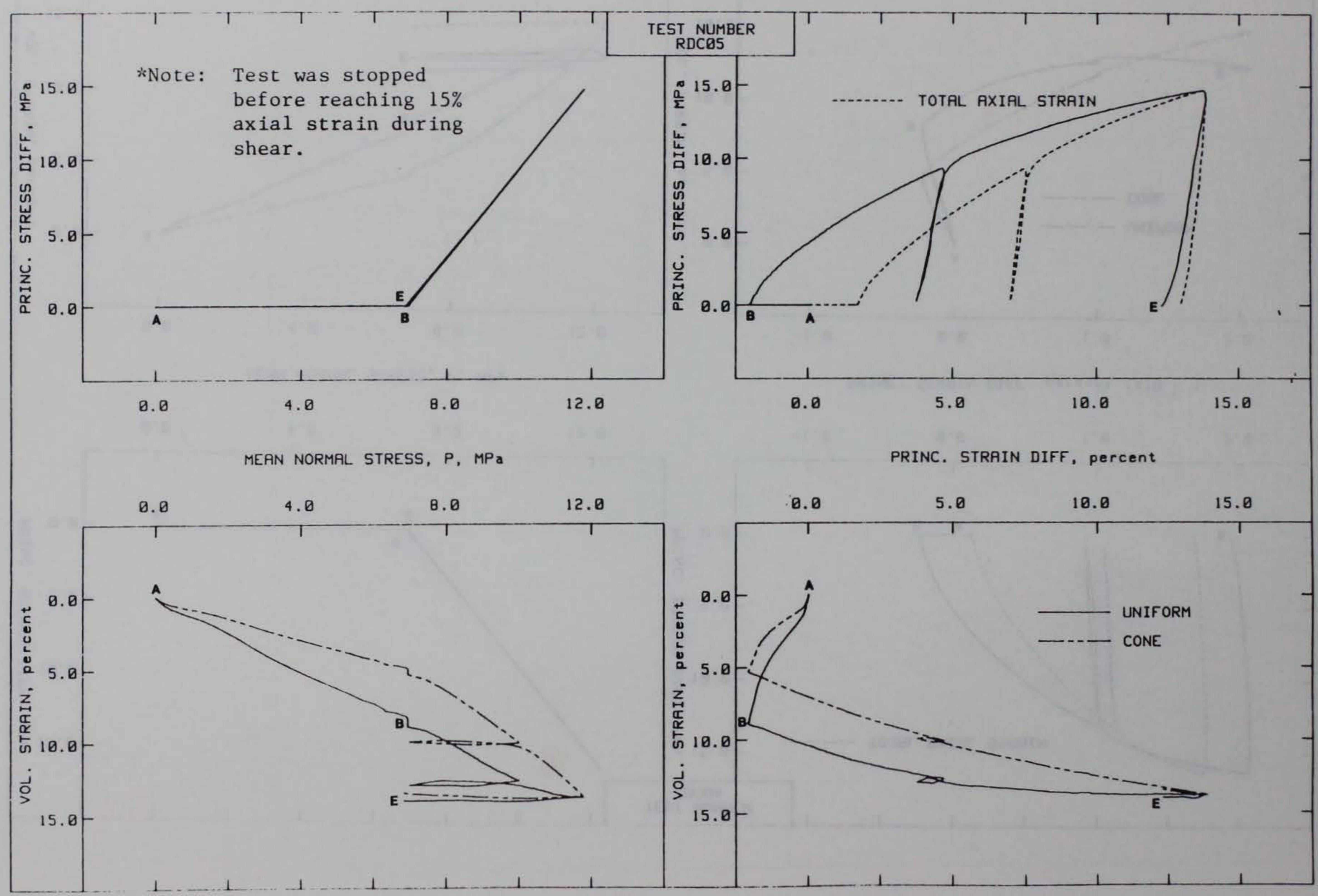




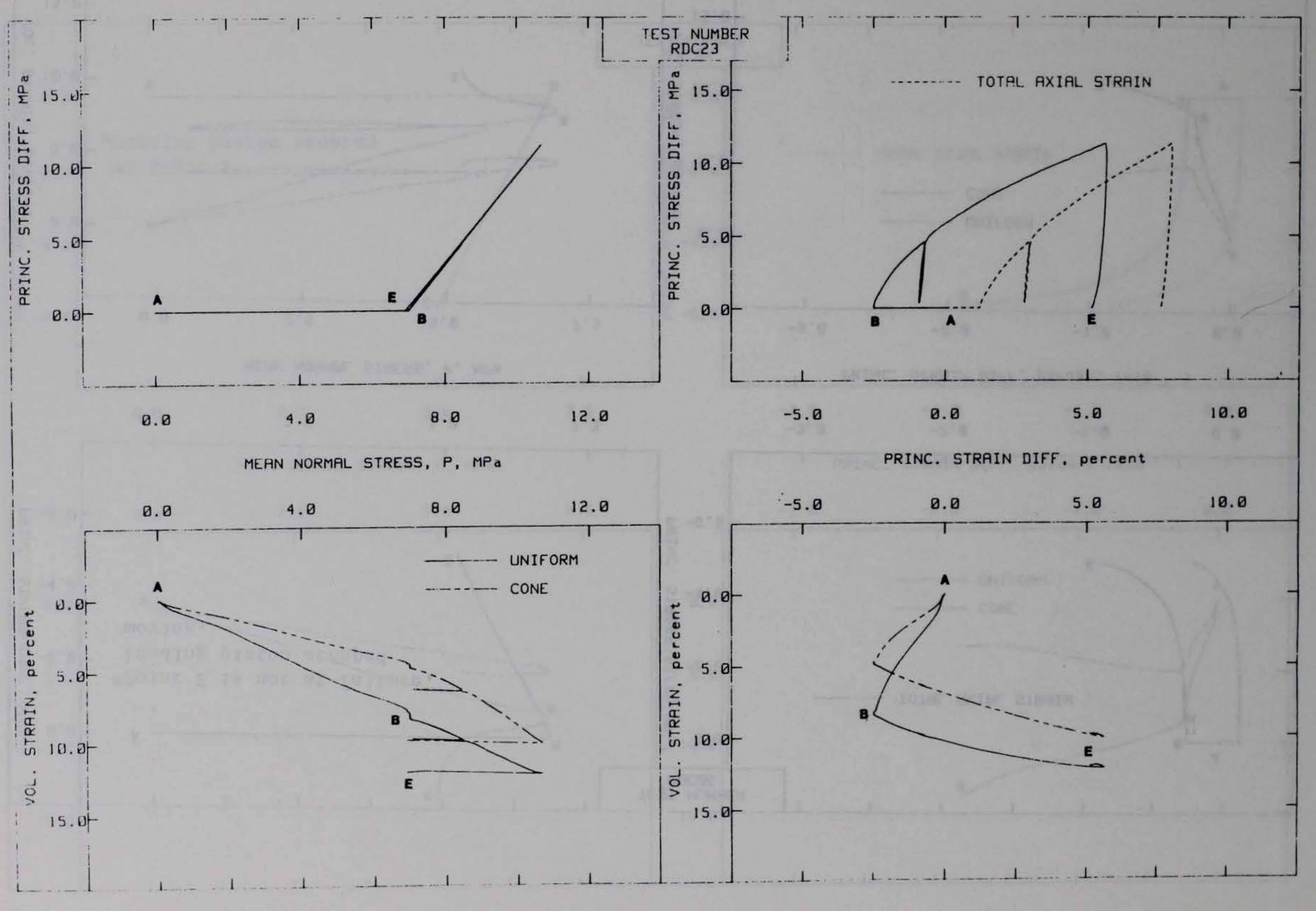




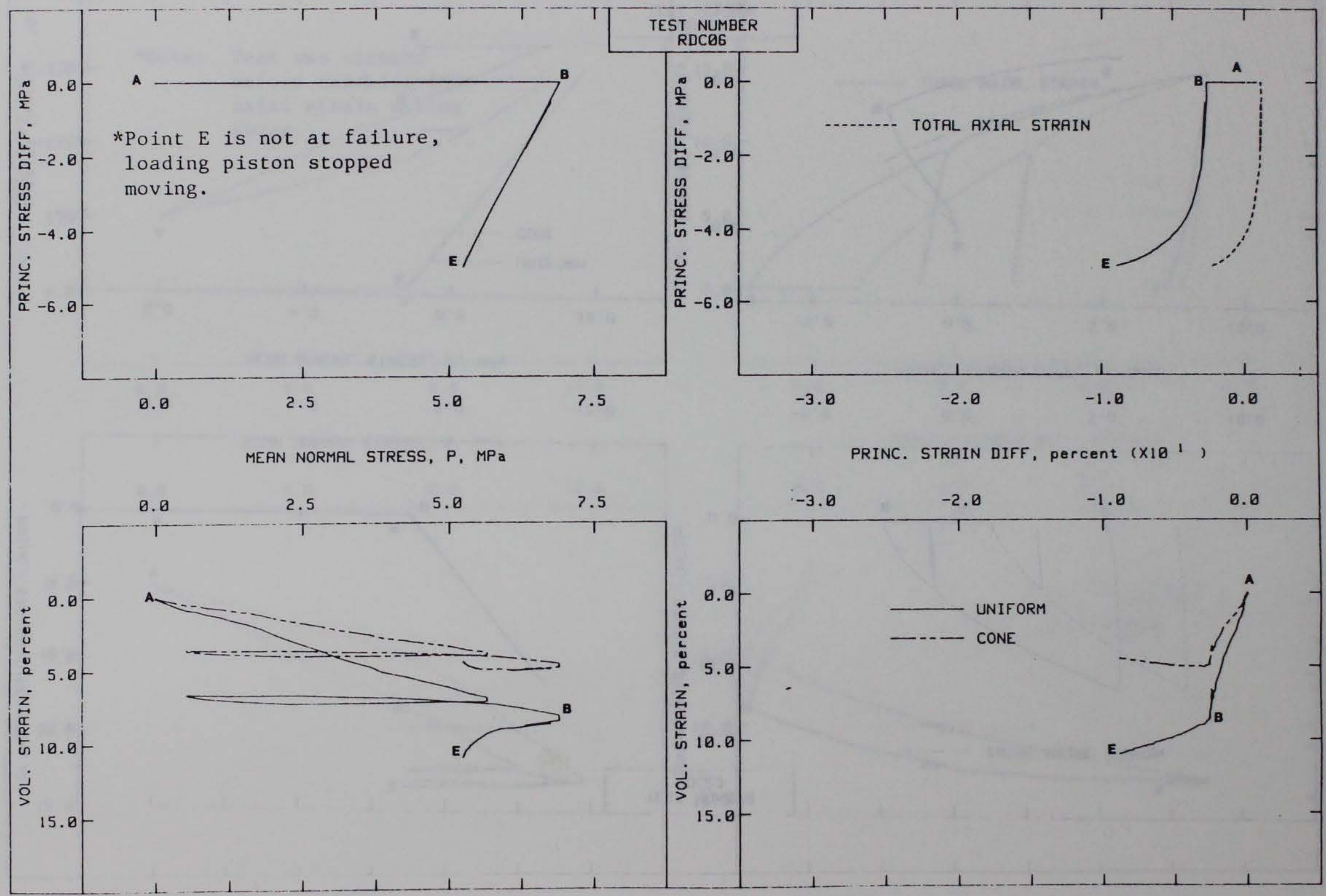




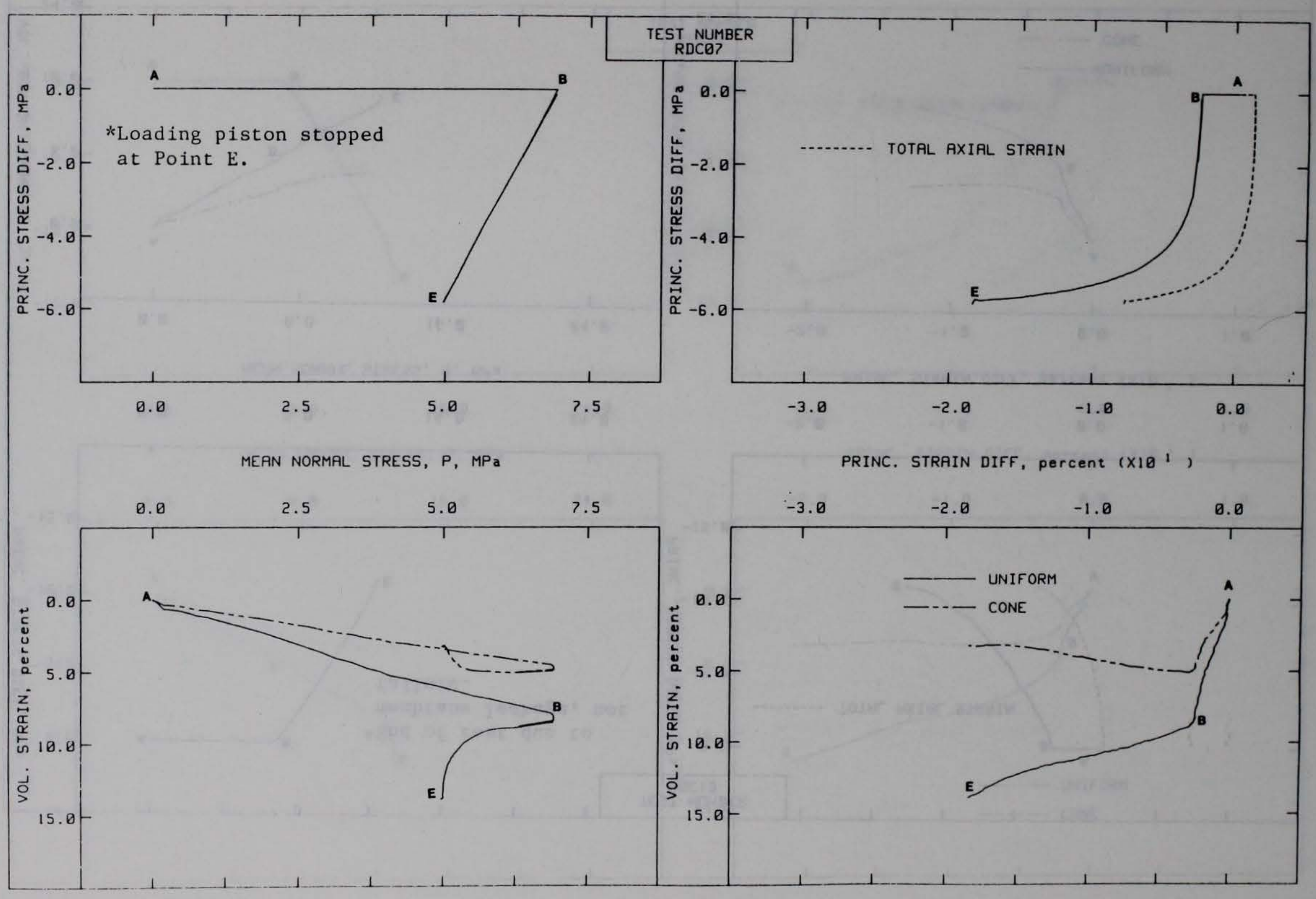




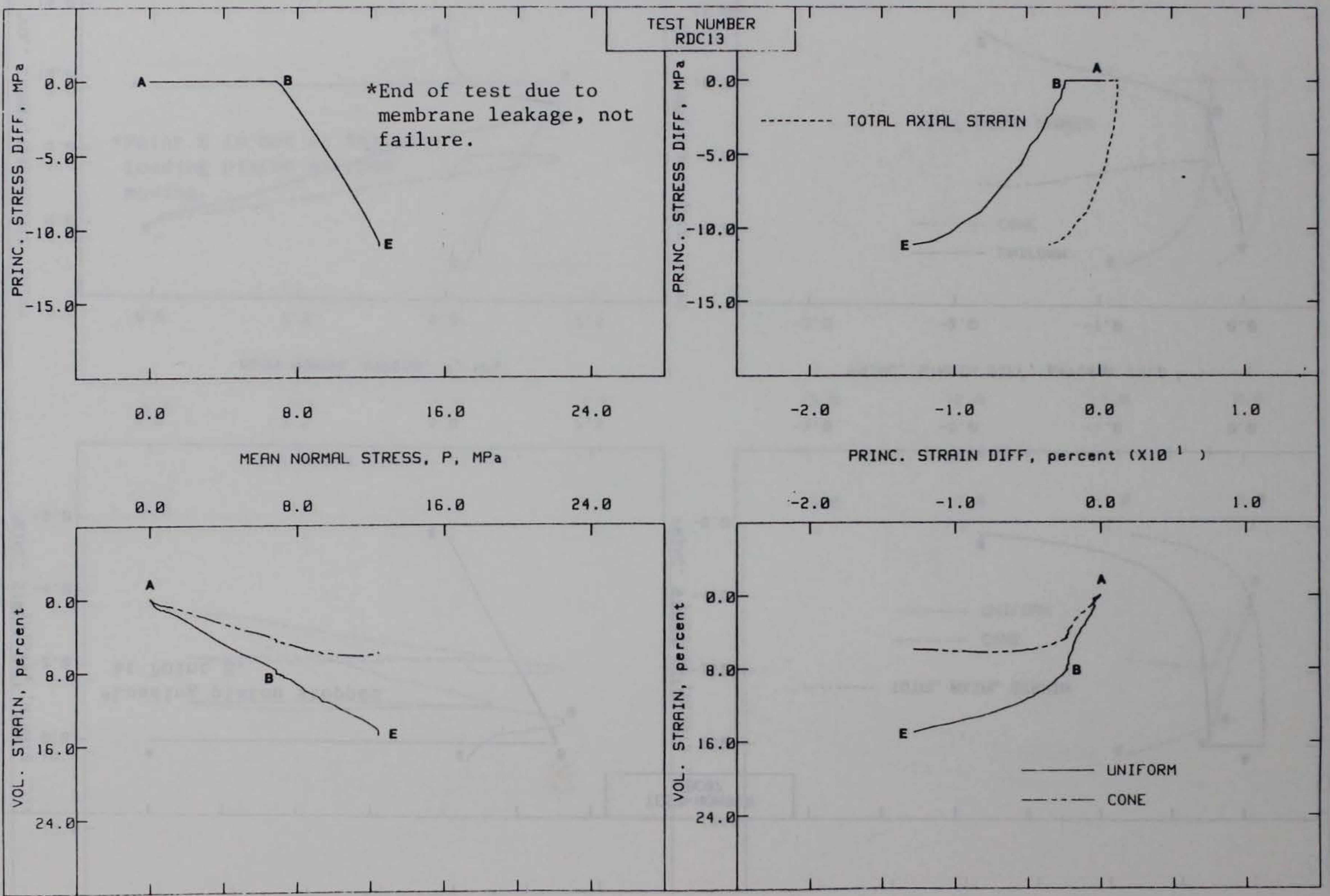




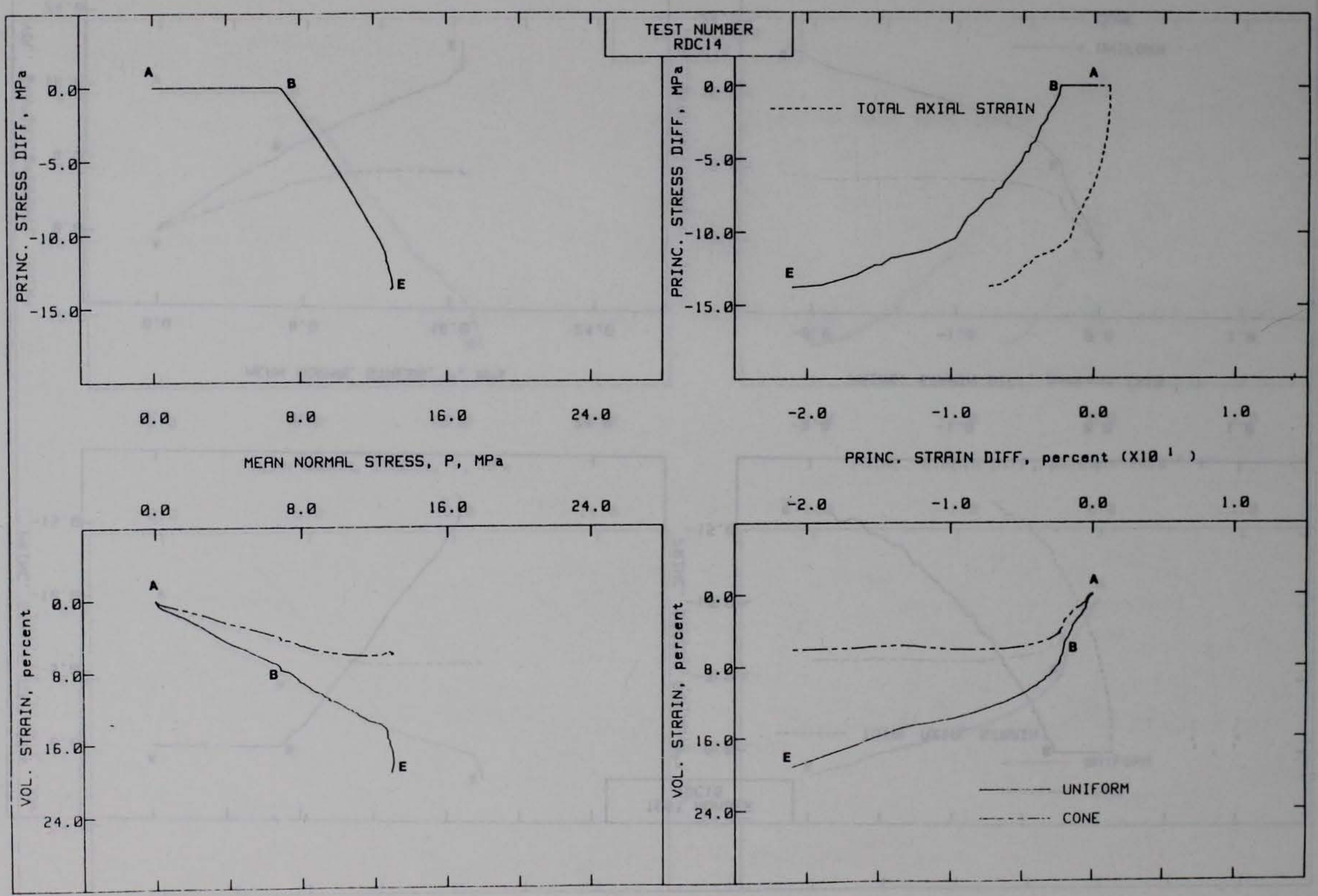




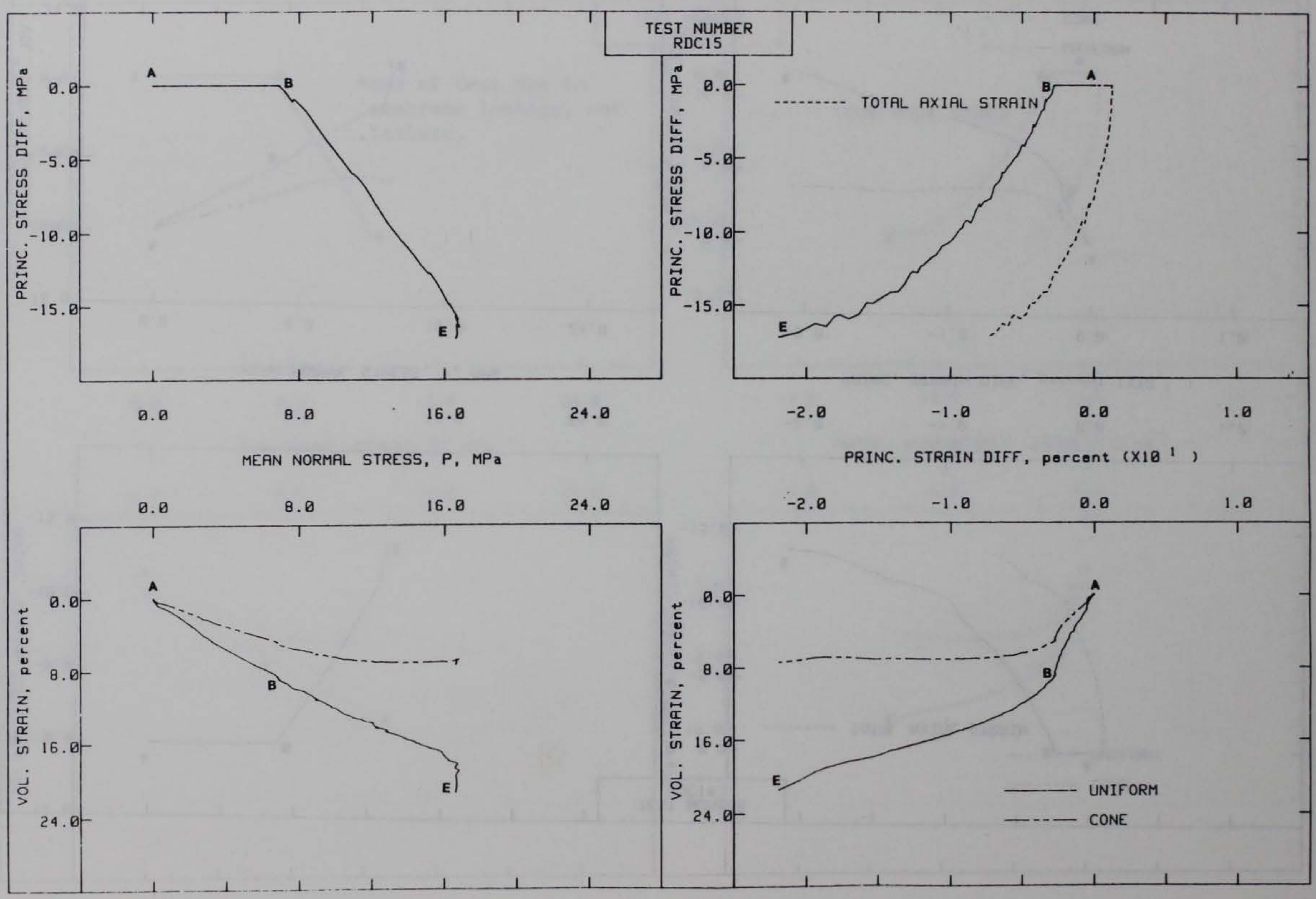




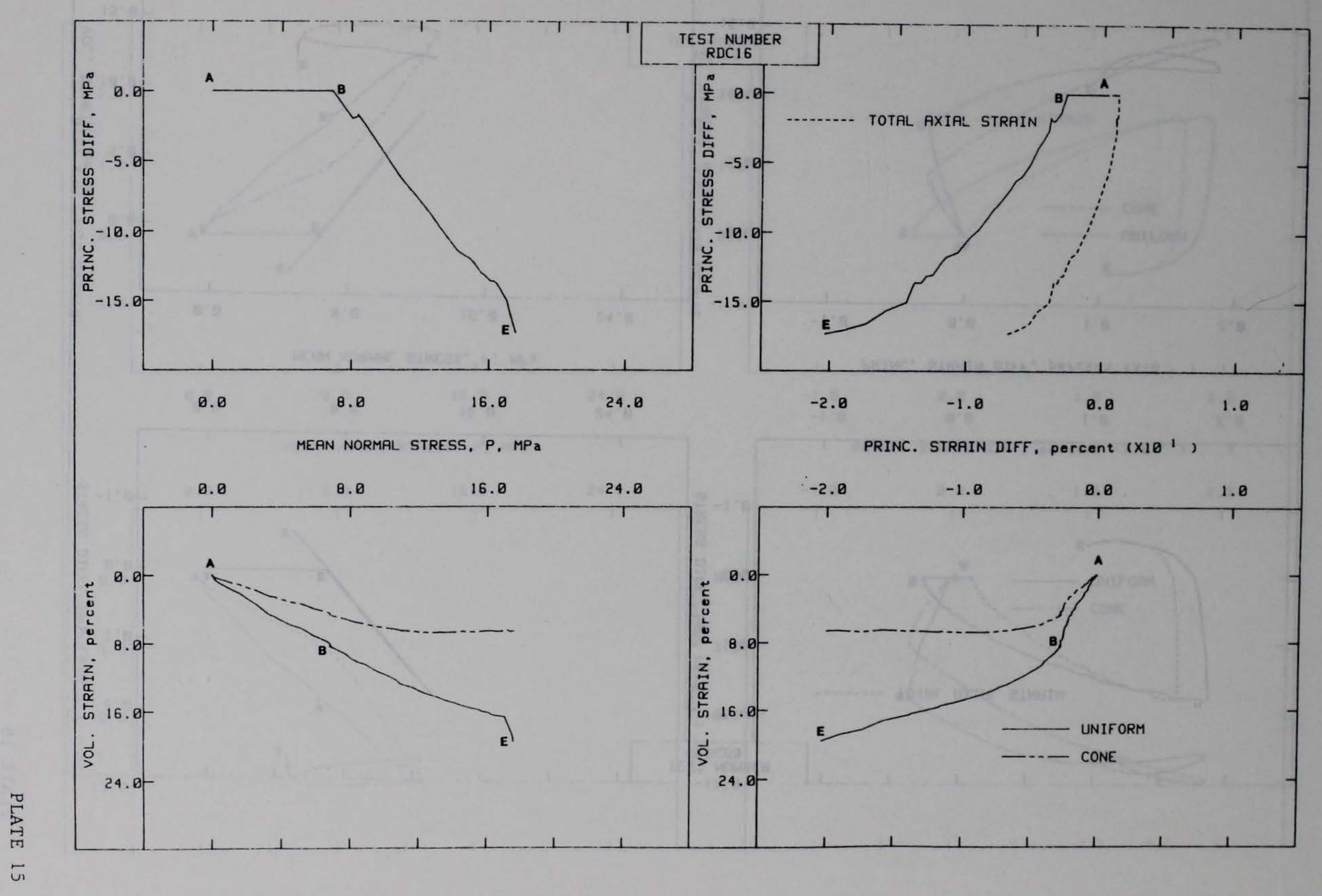



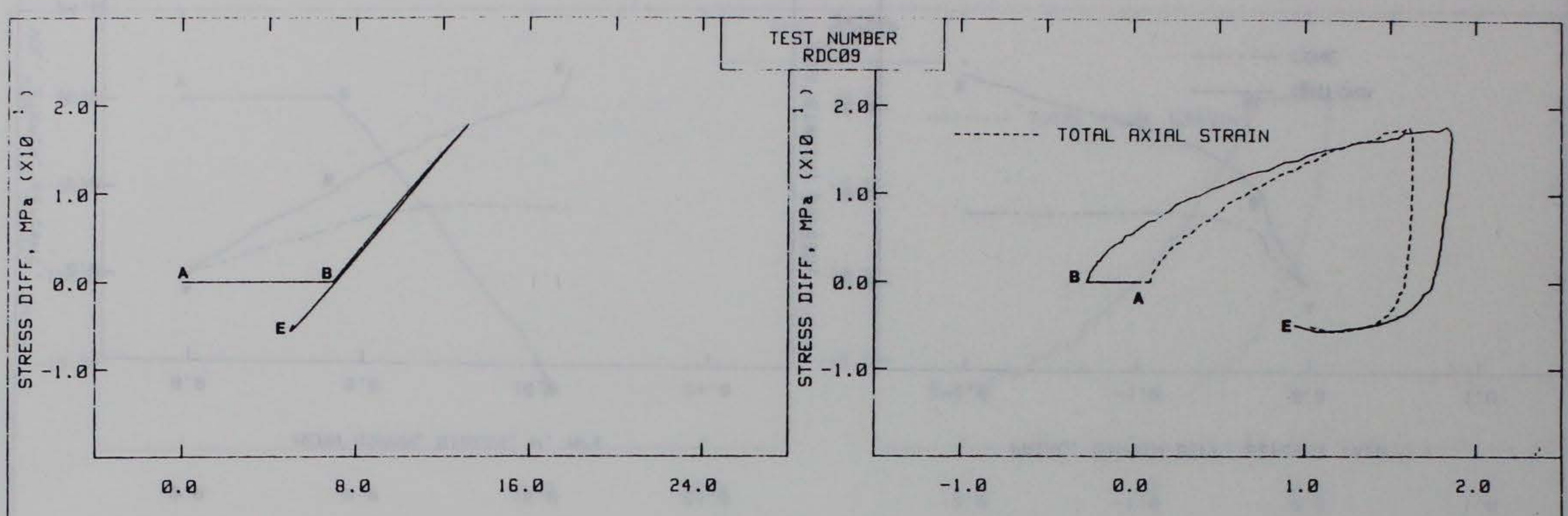

MEAN NORMAL STRESS, $P, M P a$

PRINC. STRAIN DIFF, percent $\left(X 10^{1}\right)$

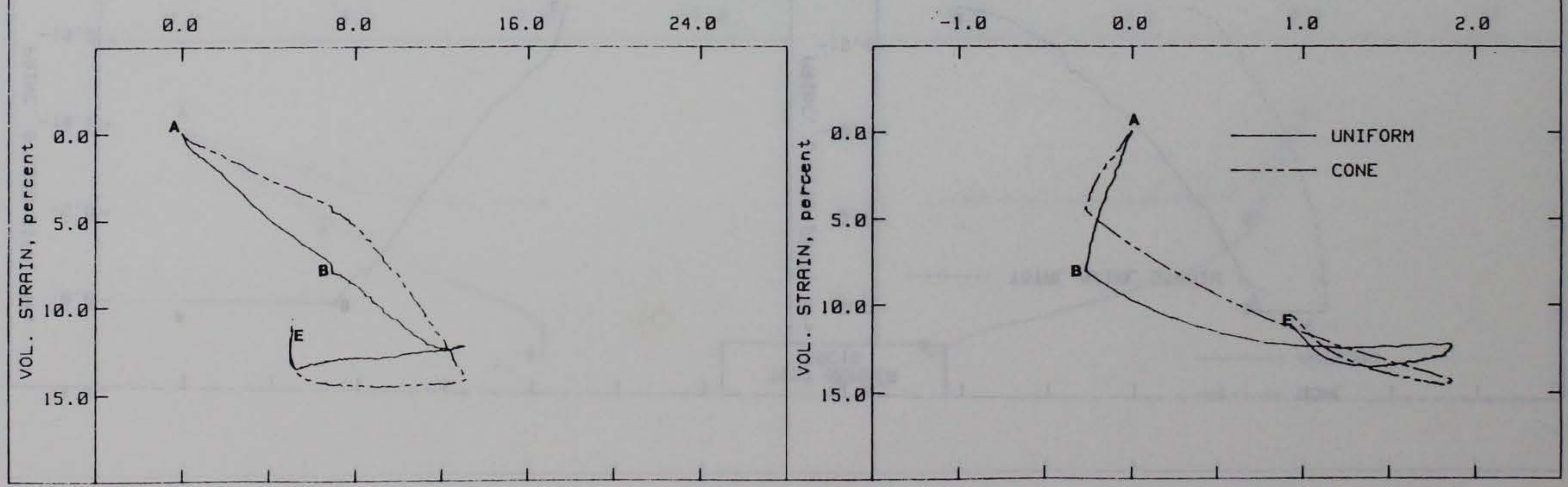




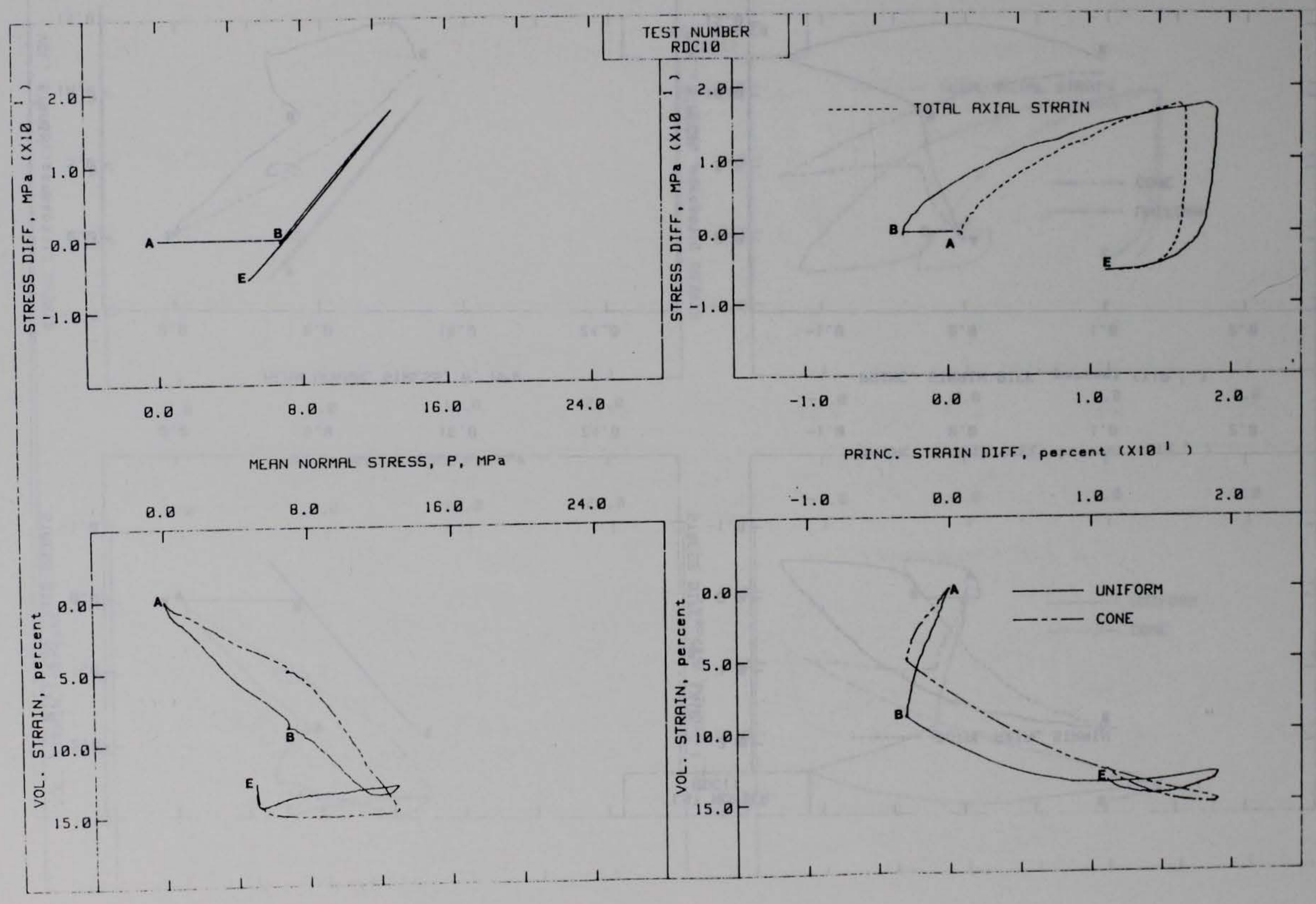




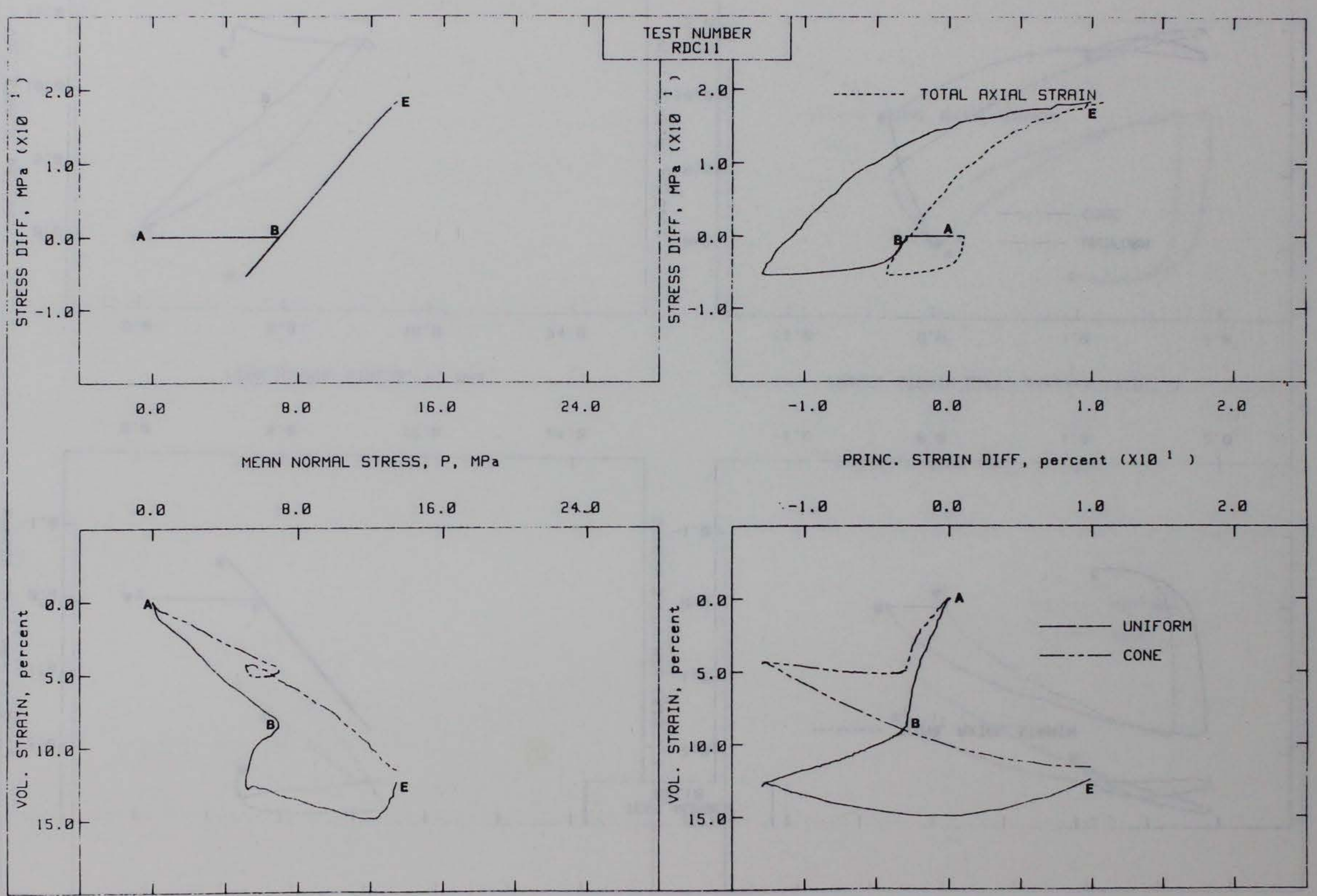



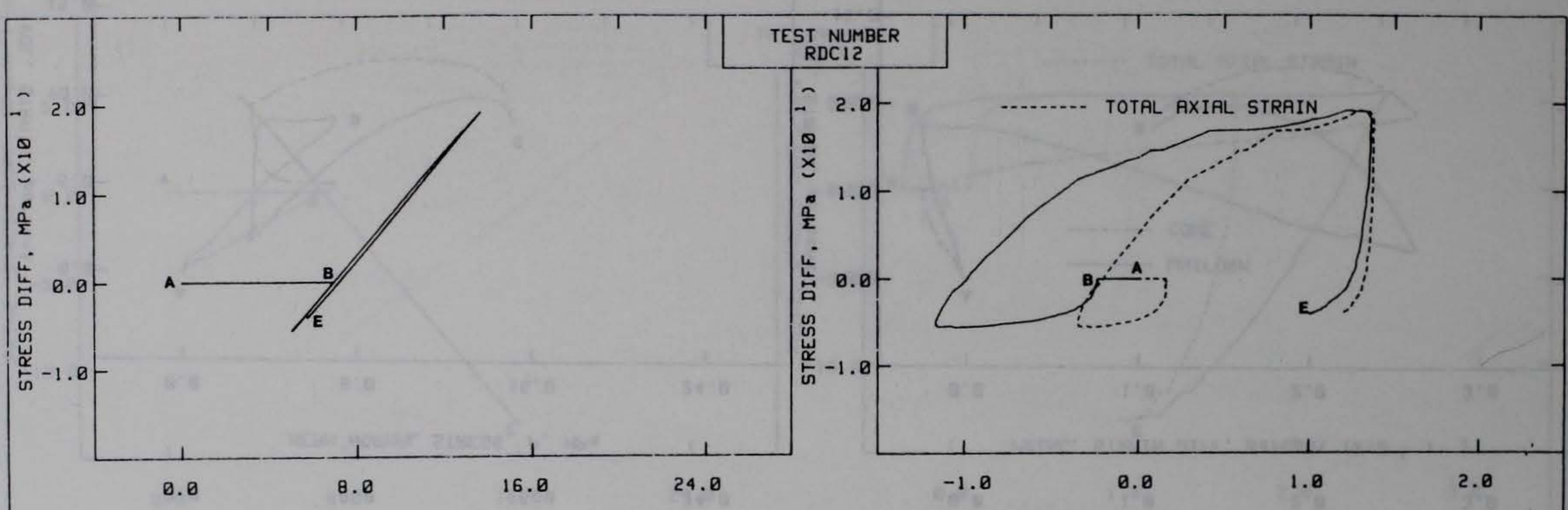

MEAN NORMAL STRESS, $P, M P a$

PRINC. STRAIN DIFF, percent $\left(X_{10} 0^{1}\right)$
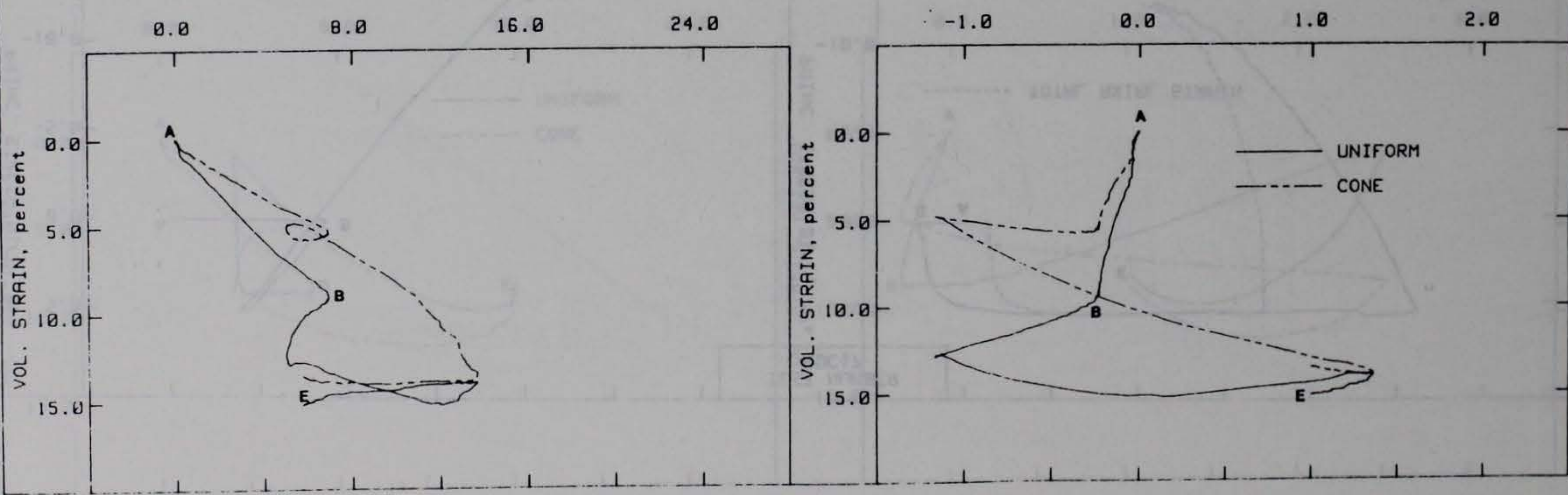



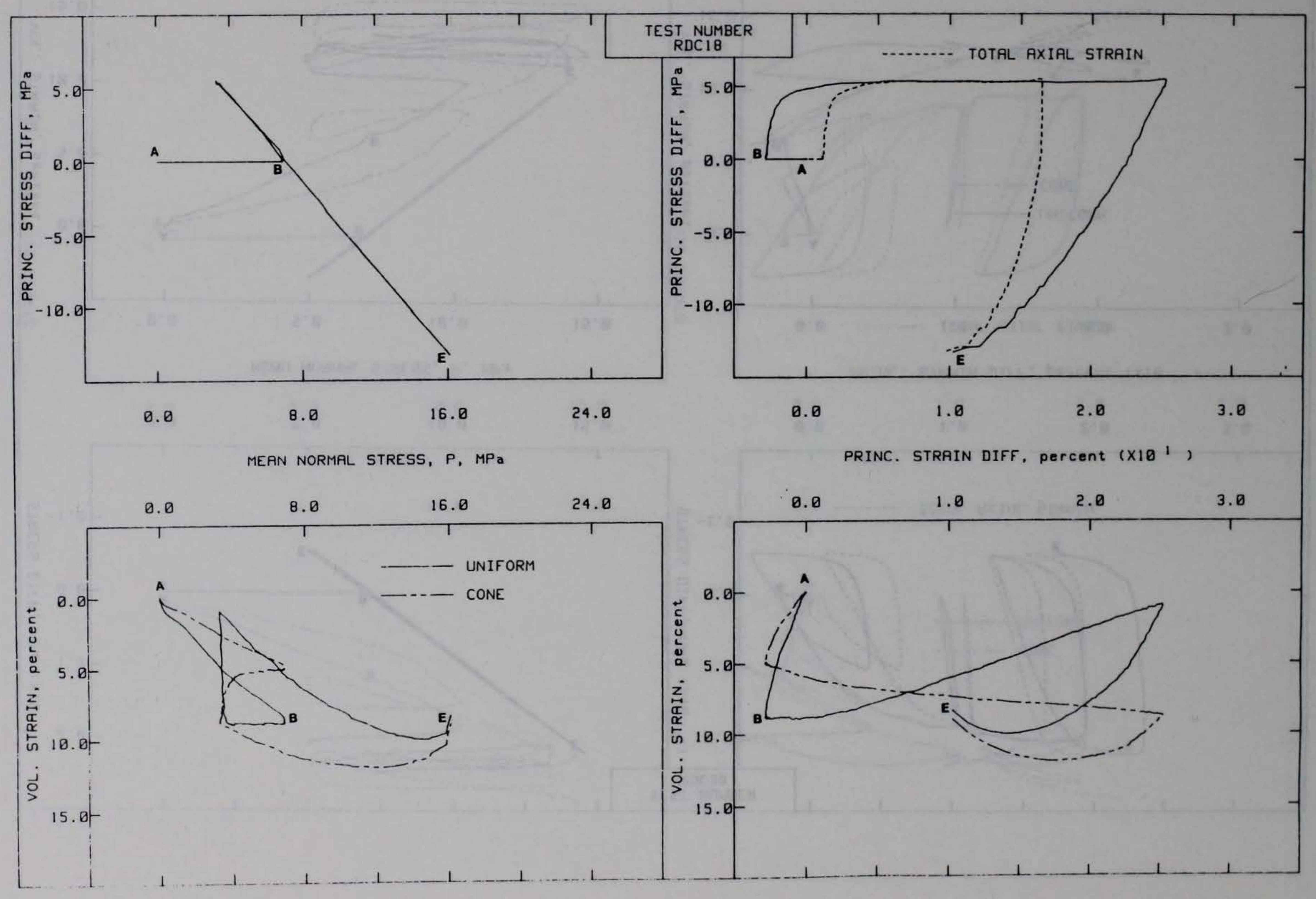




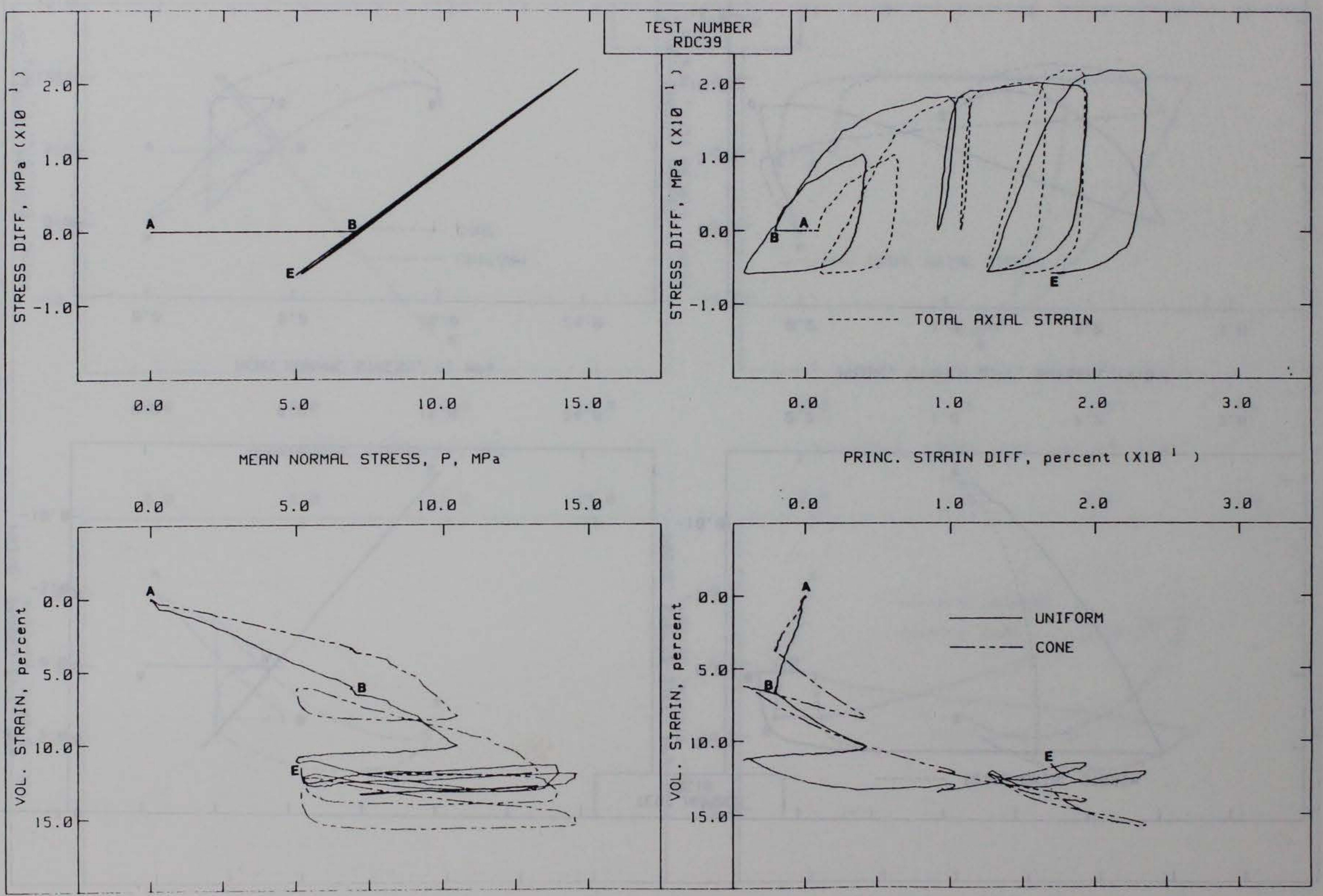




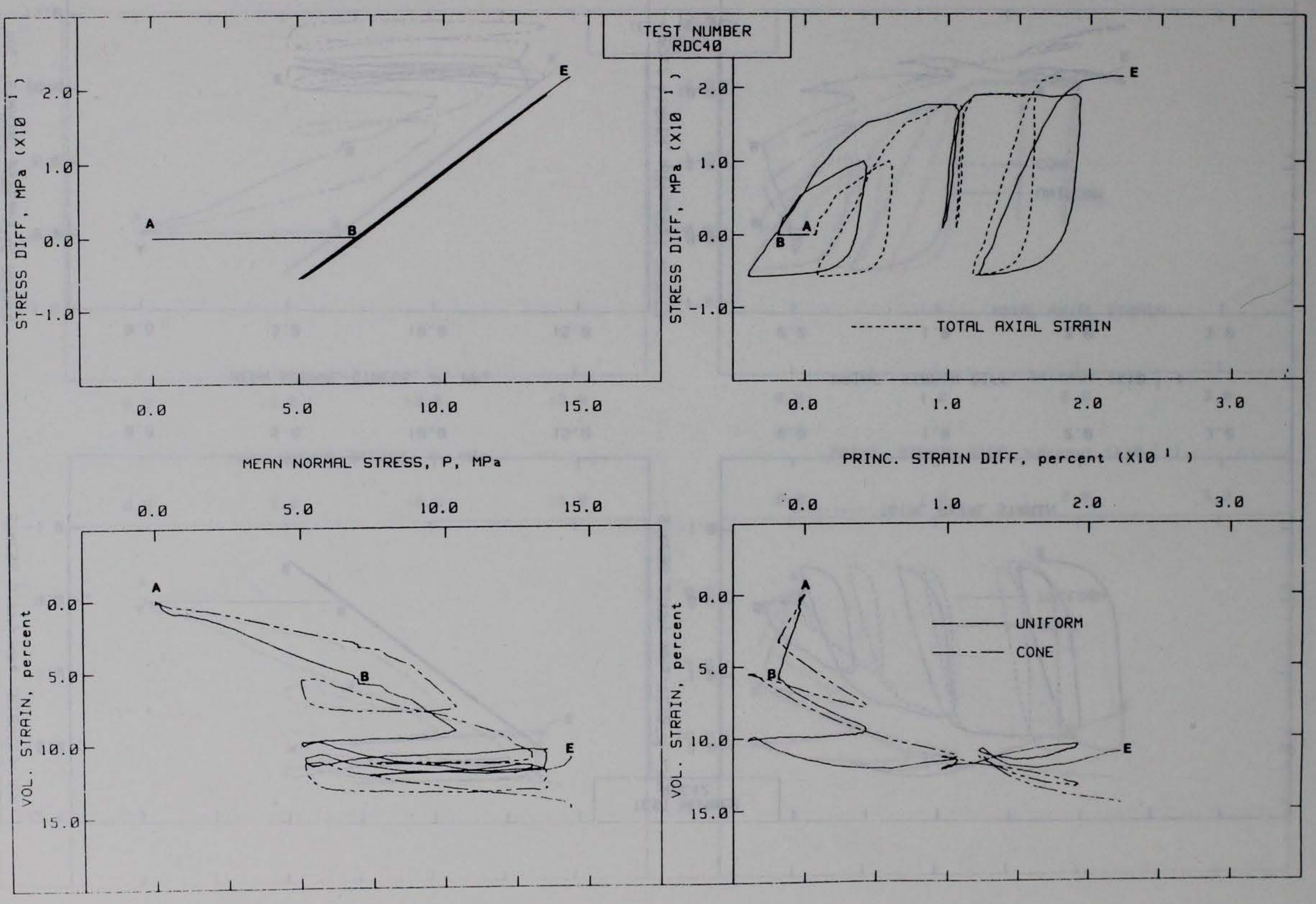




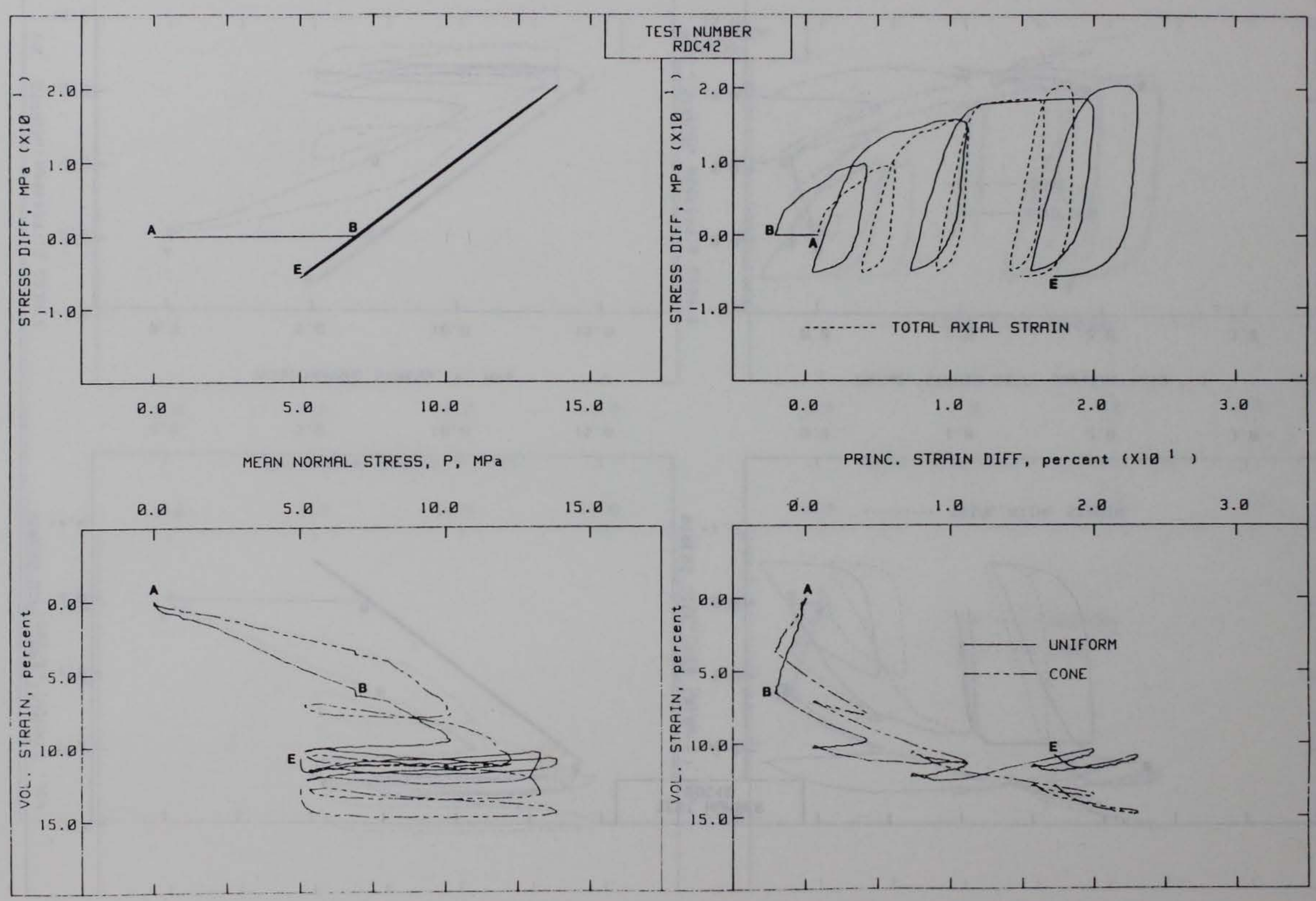




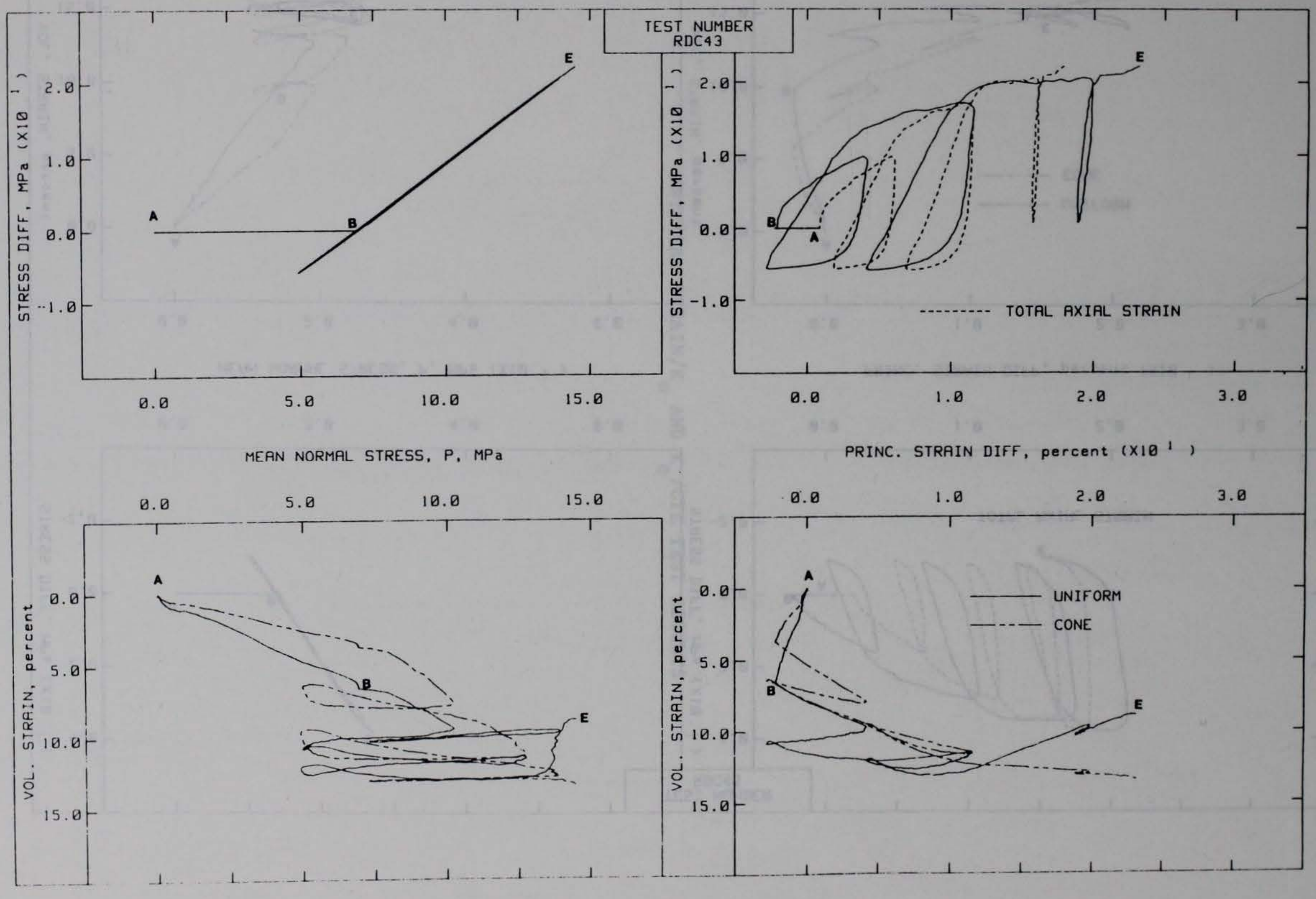




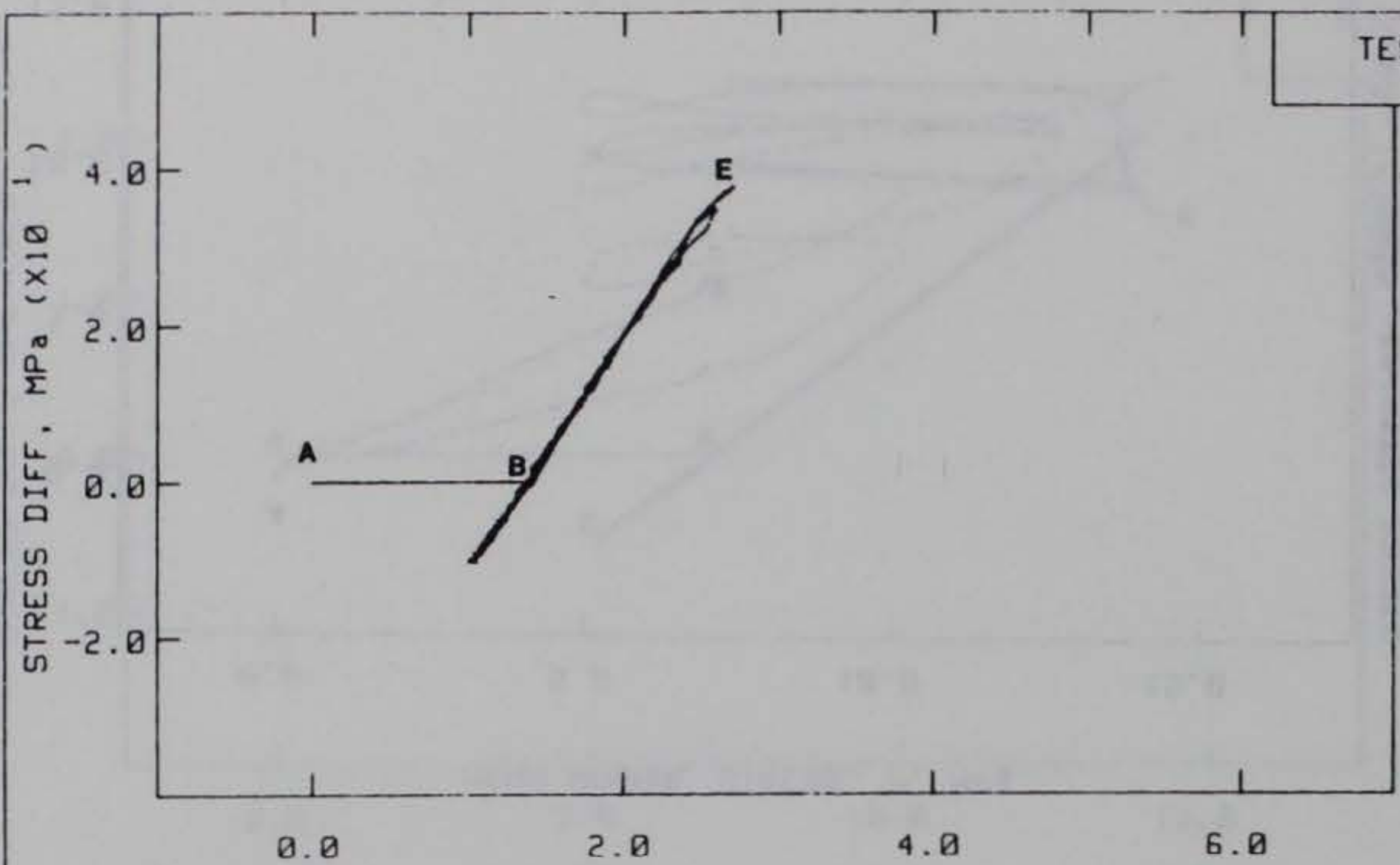

TEST NUMBER
RDC 45

MEAN NORMAL STRESS, $\mathrm{P}, \mathrm{MPa}\left(\mathrm{X}_{10}{ }^{\prime}\right)$

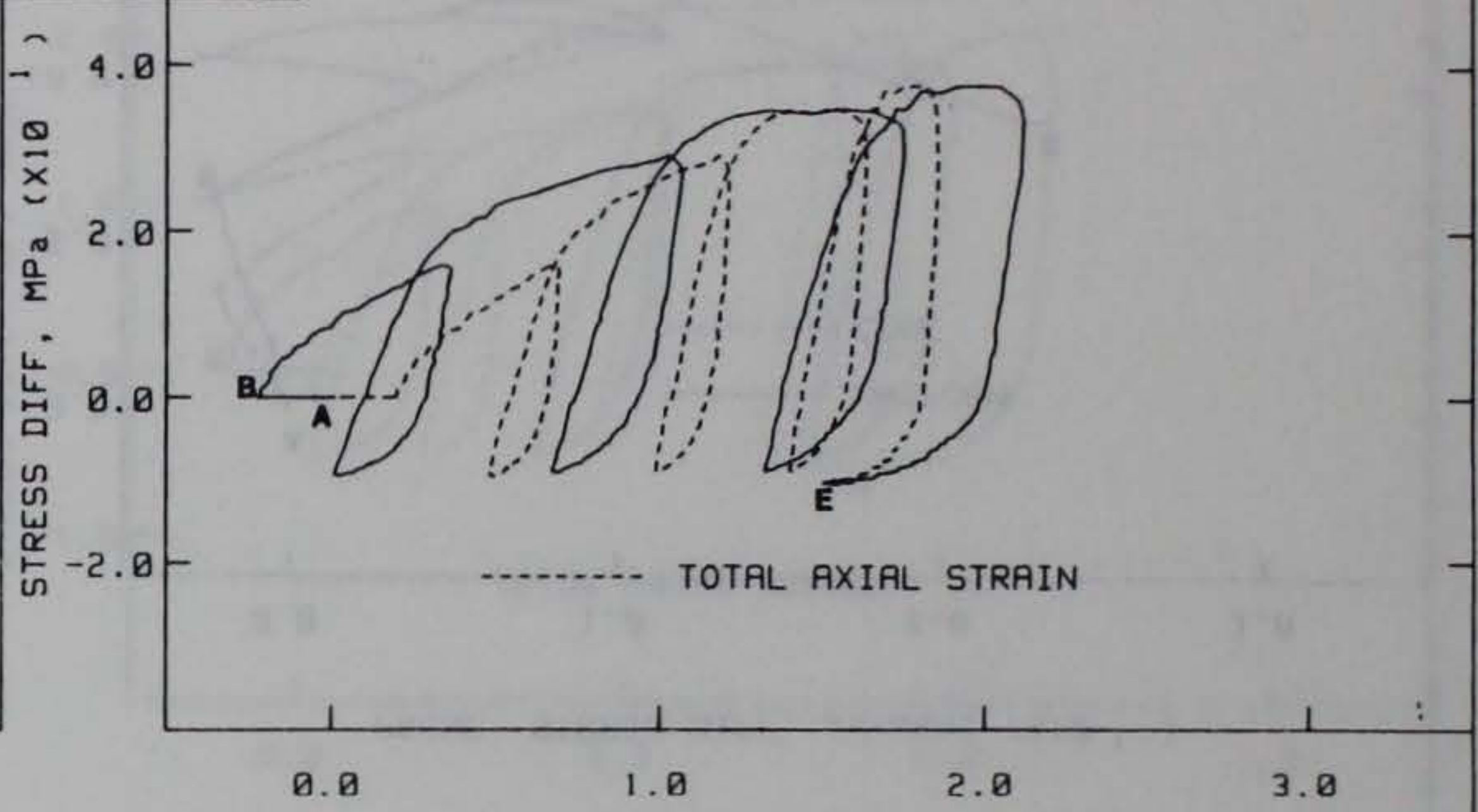

PRINC. STRAIN DIFF, percent $\left(X_{1} 0^{1}\right)$

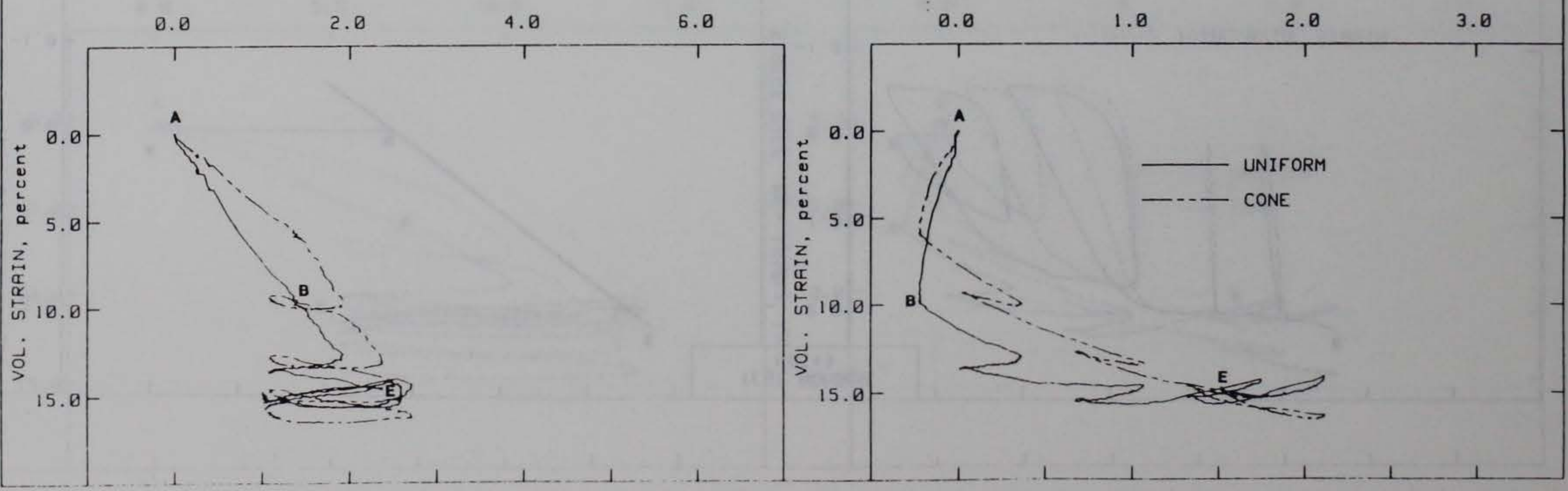


UNIAXIAL STRAIN/K $\mathrm{K}_{0}$ AND $\mathrm{K}_{\mathrm{o}} /$ CTE TEST RESULTS 


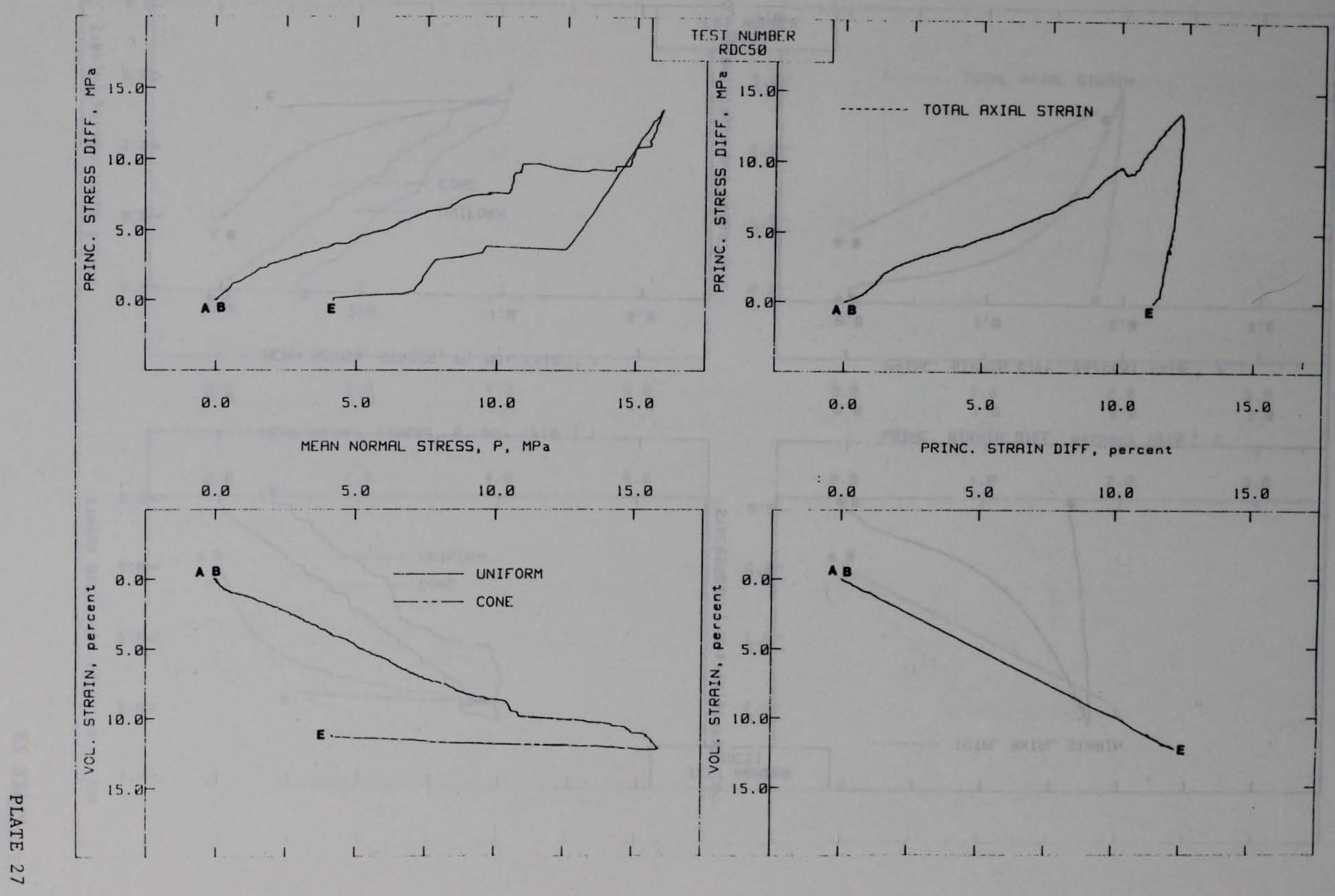




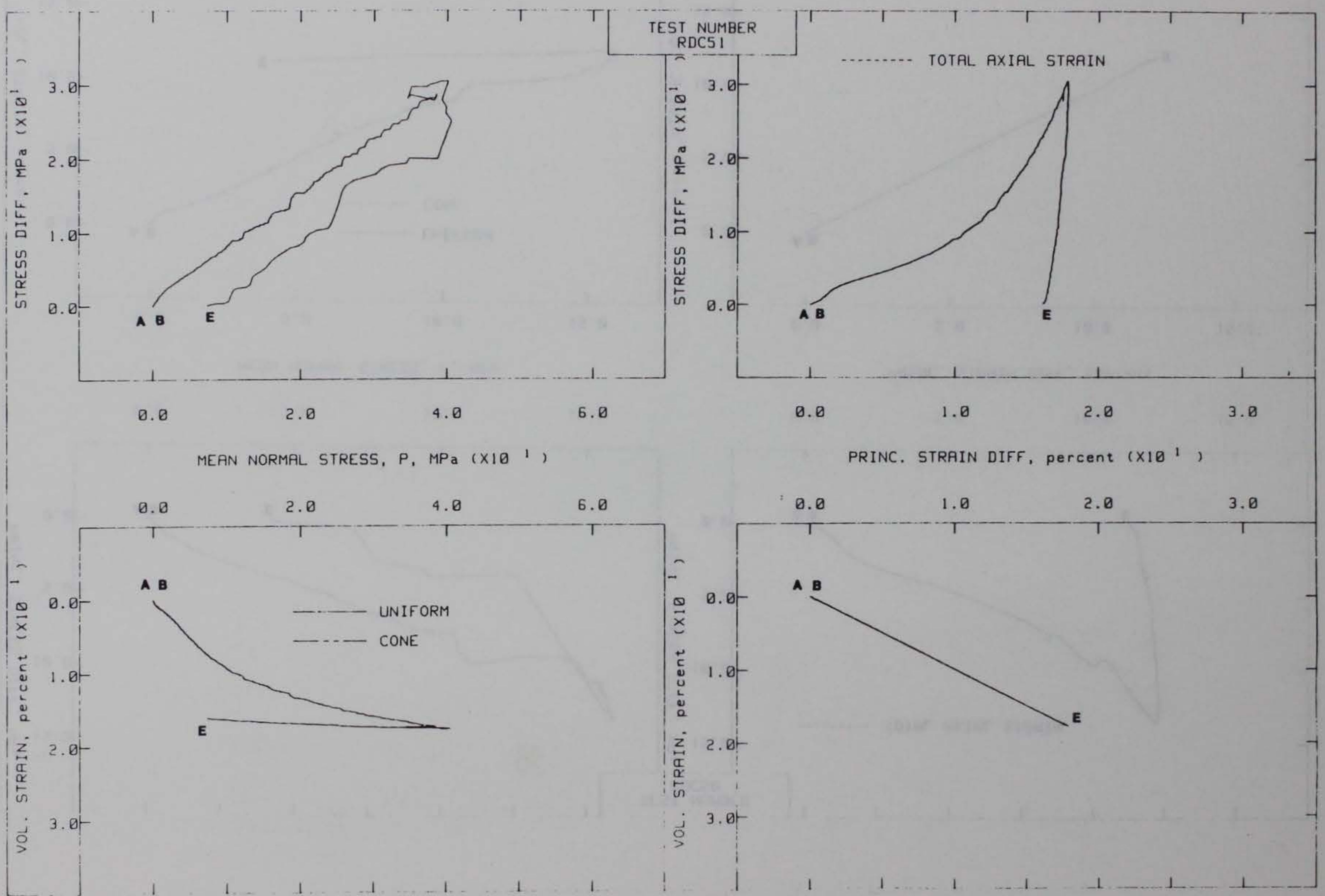




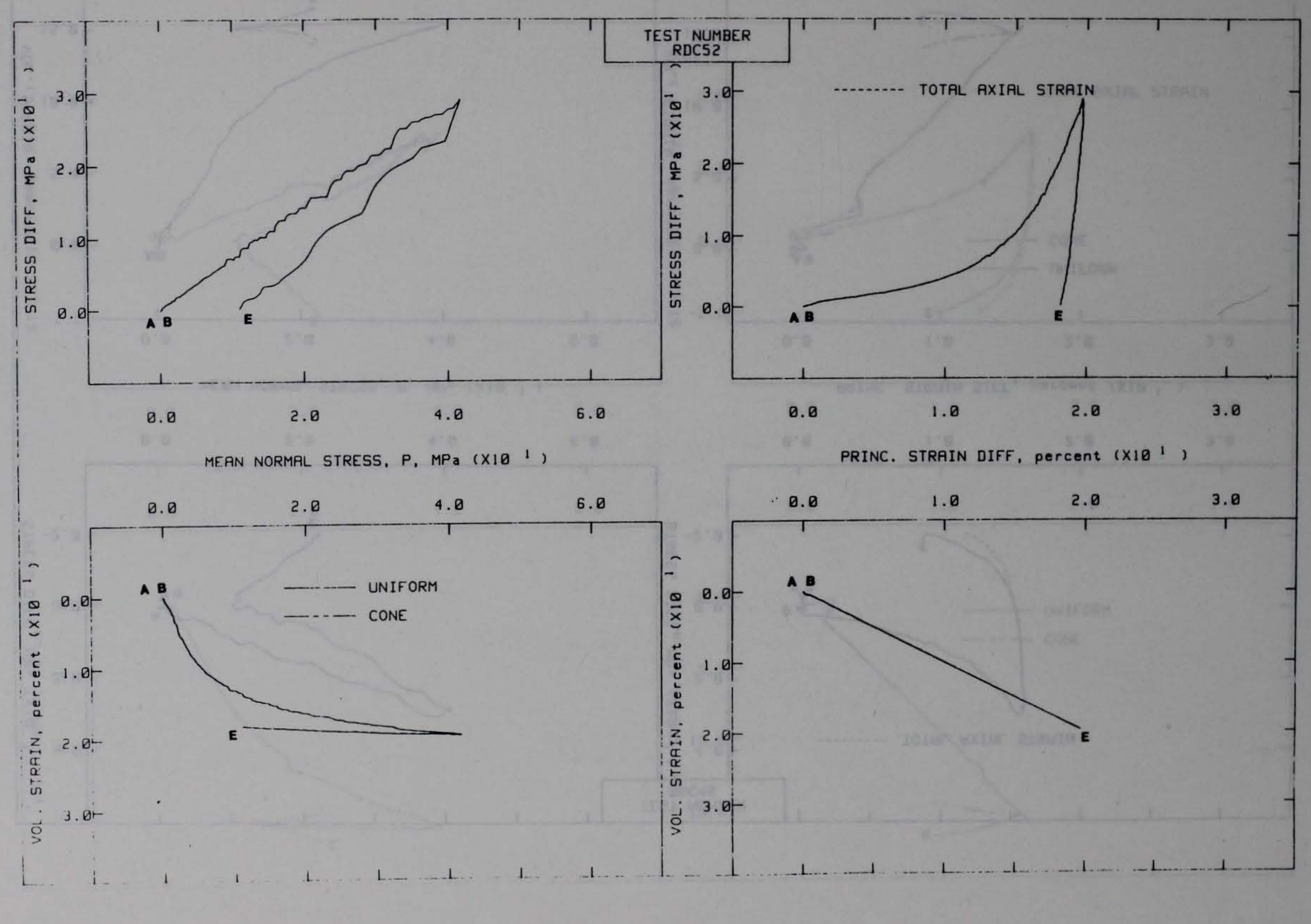




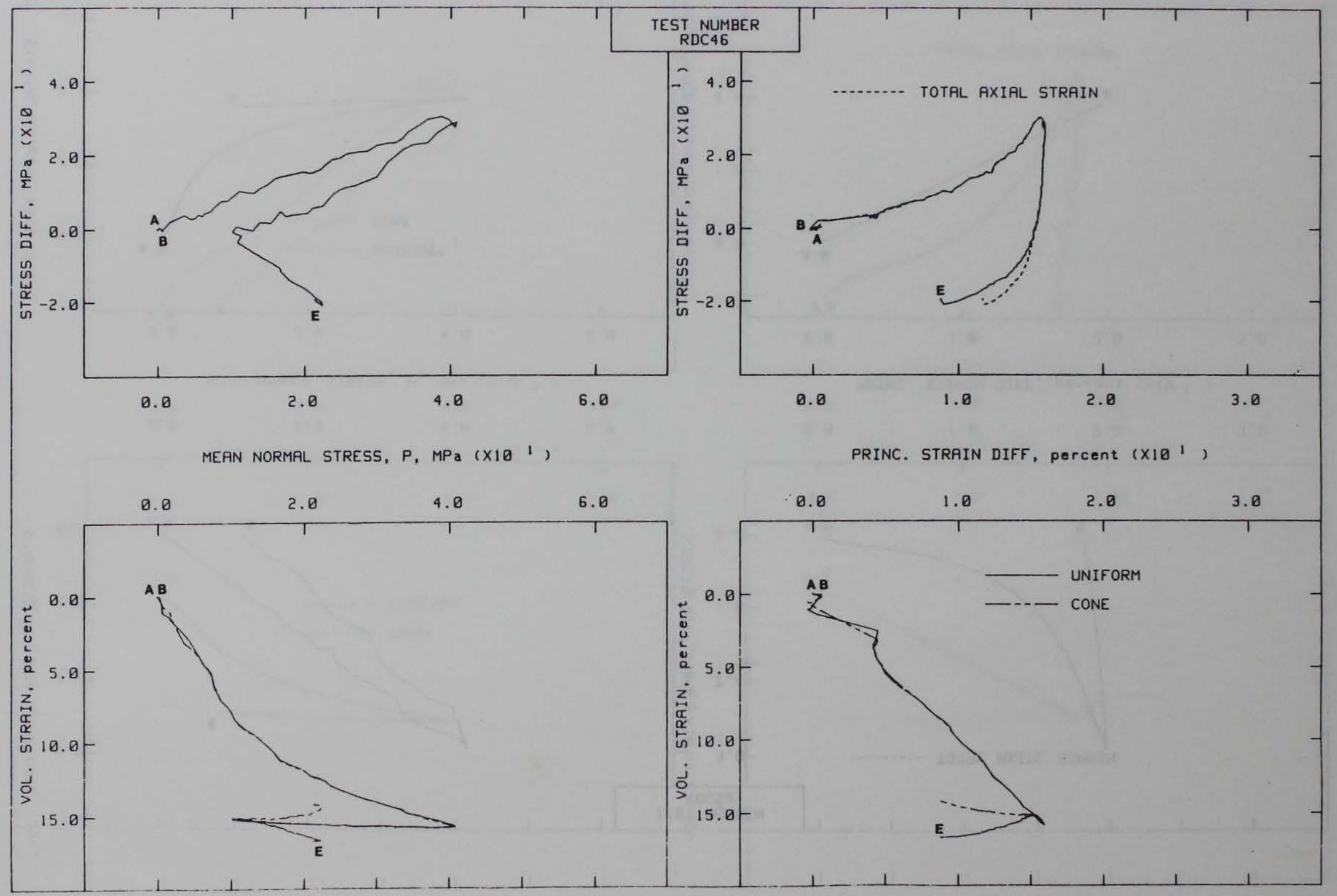




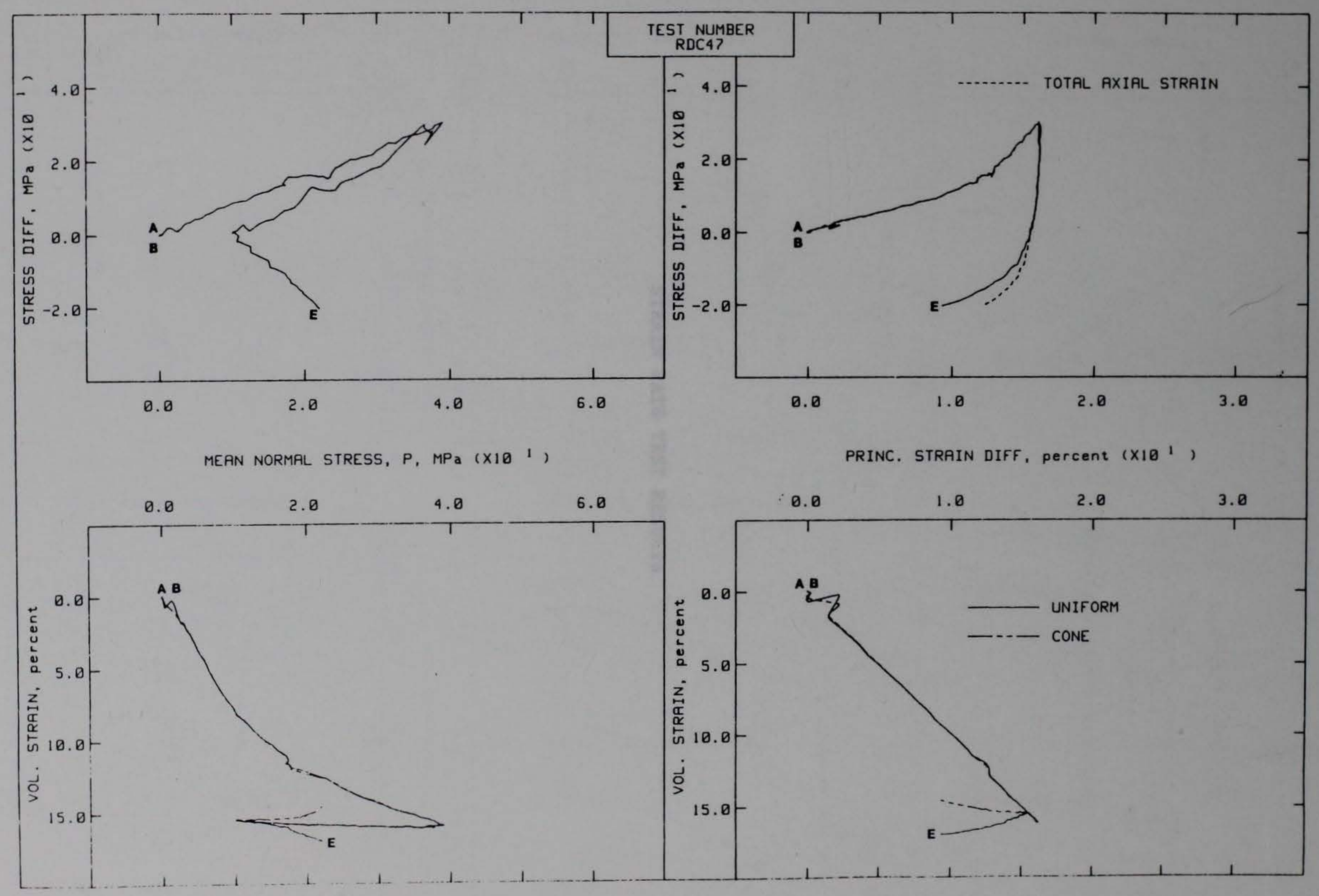


STRAIN PATH TEST RESULTS 


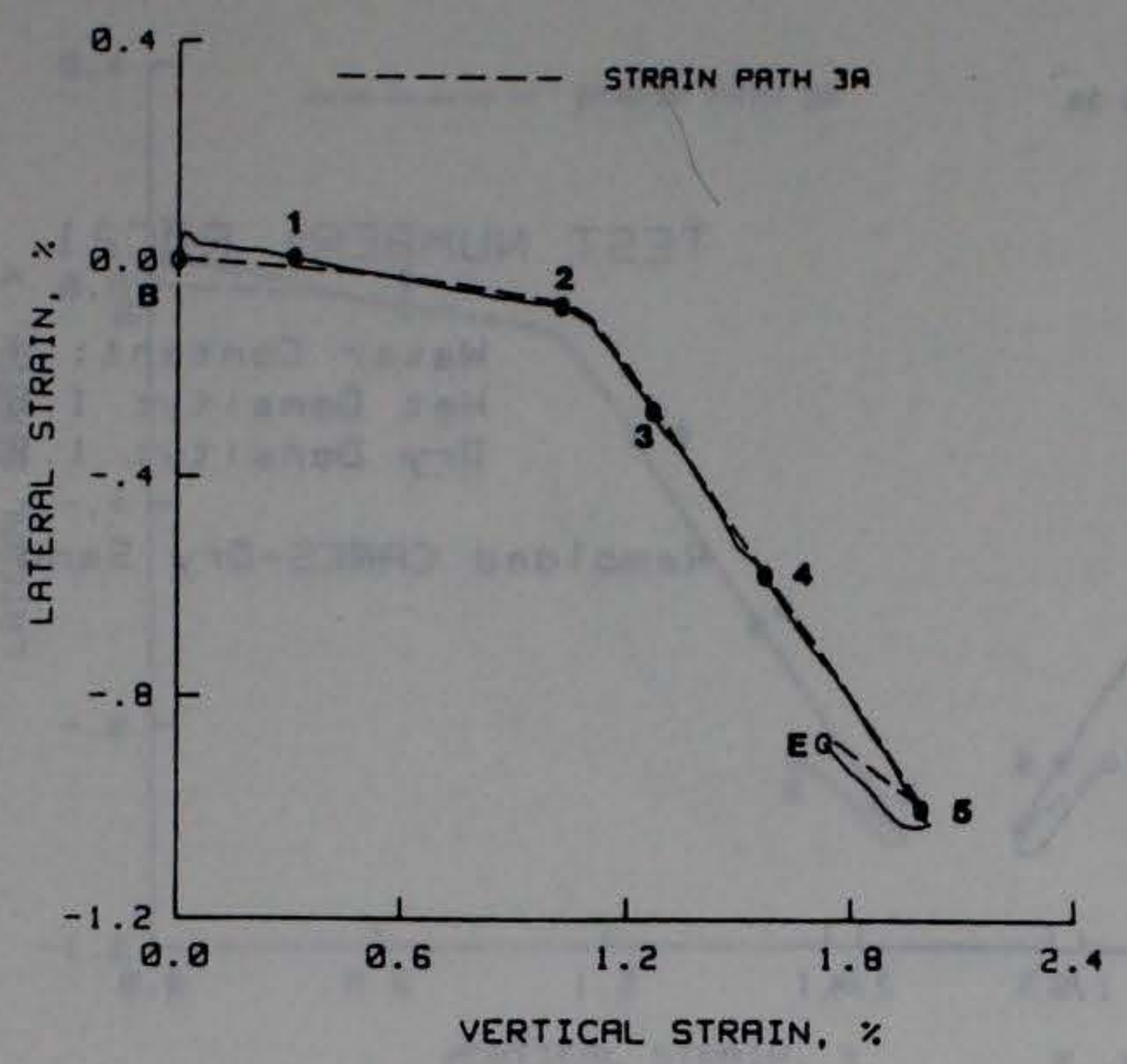

TEST NUMBER: RDCZO

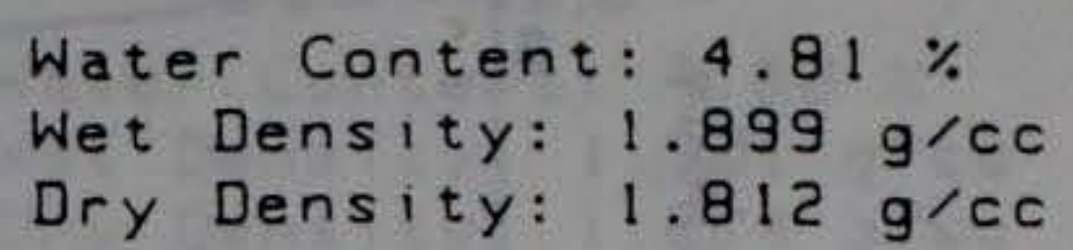

Remolded CARES-Dry Sand
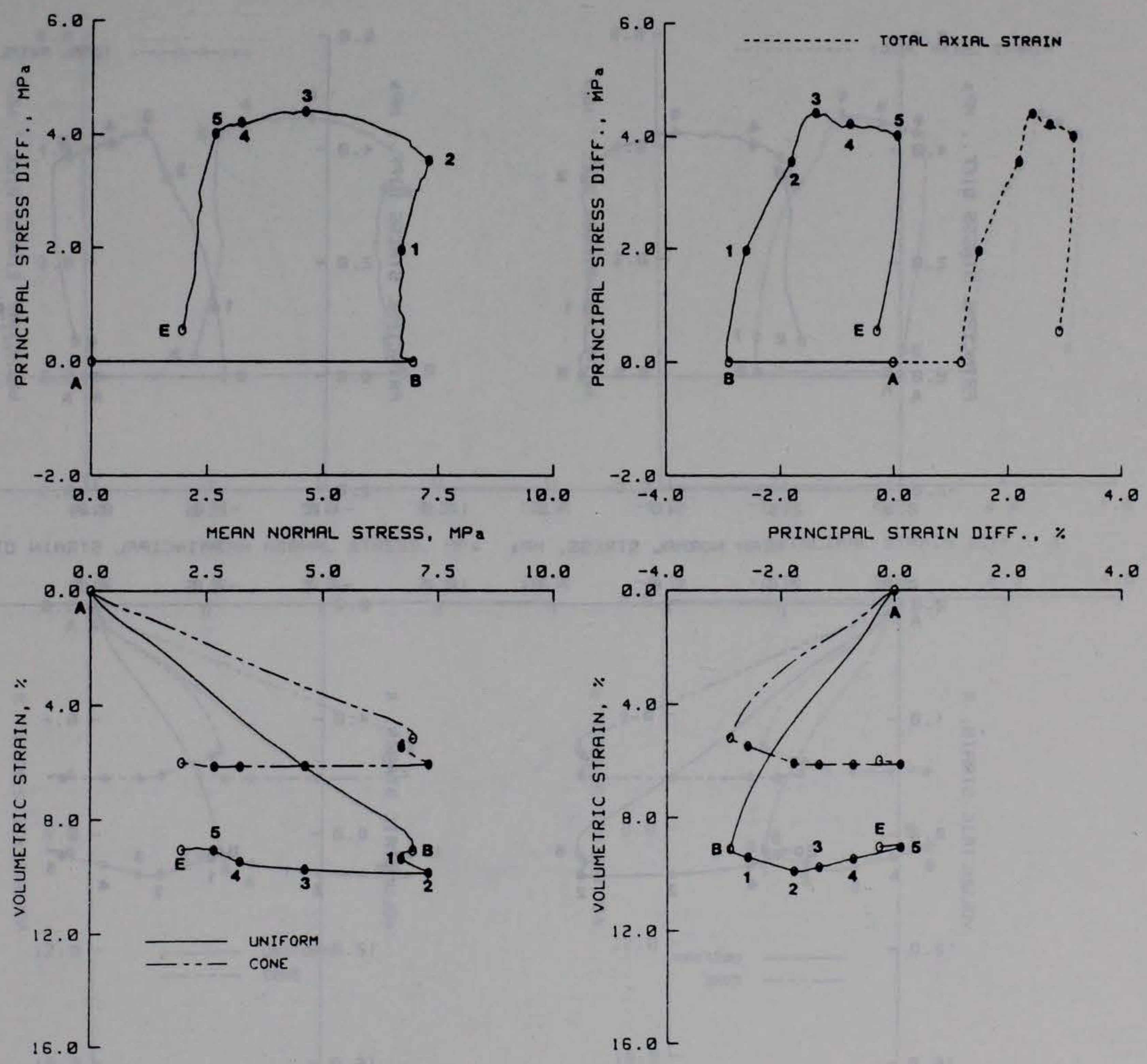


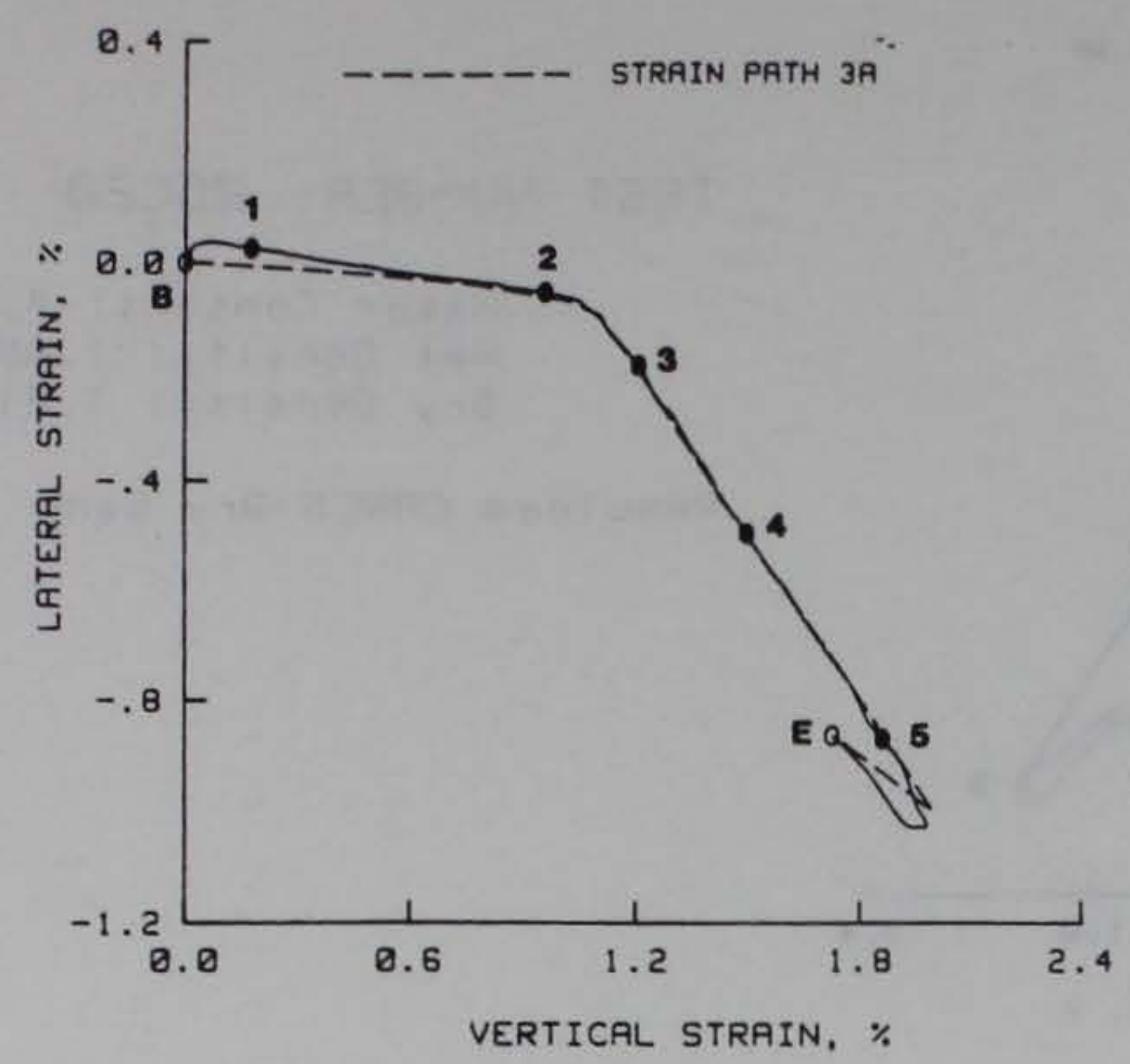

TEST NUMBER: RDC2 1

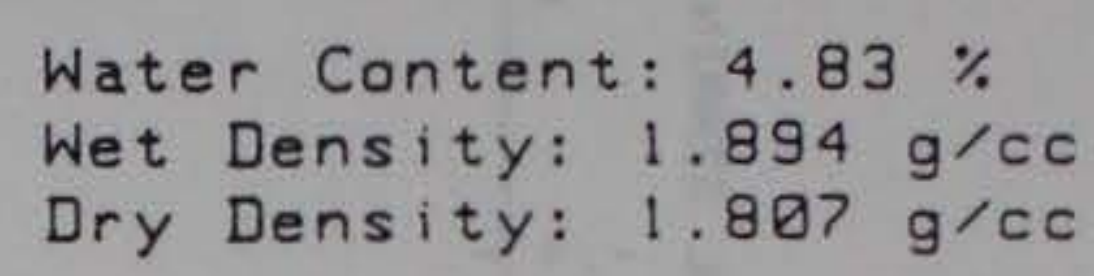

Remolded CARES-Dry Sand
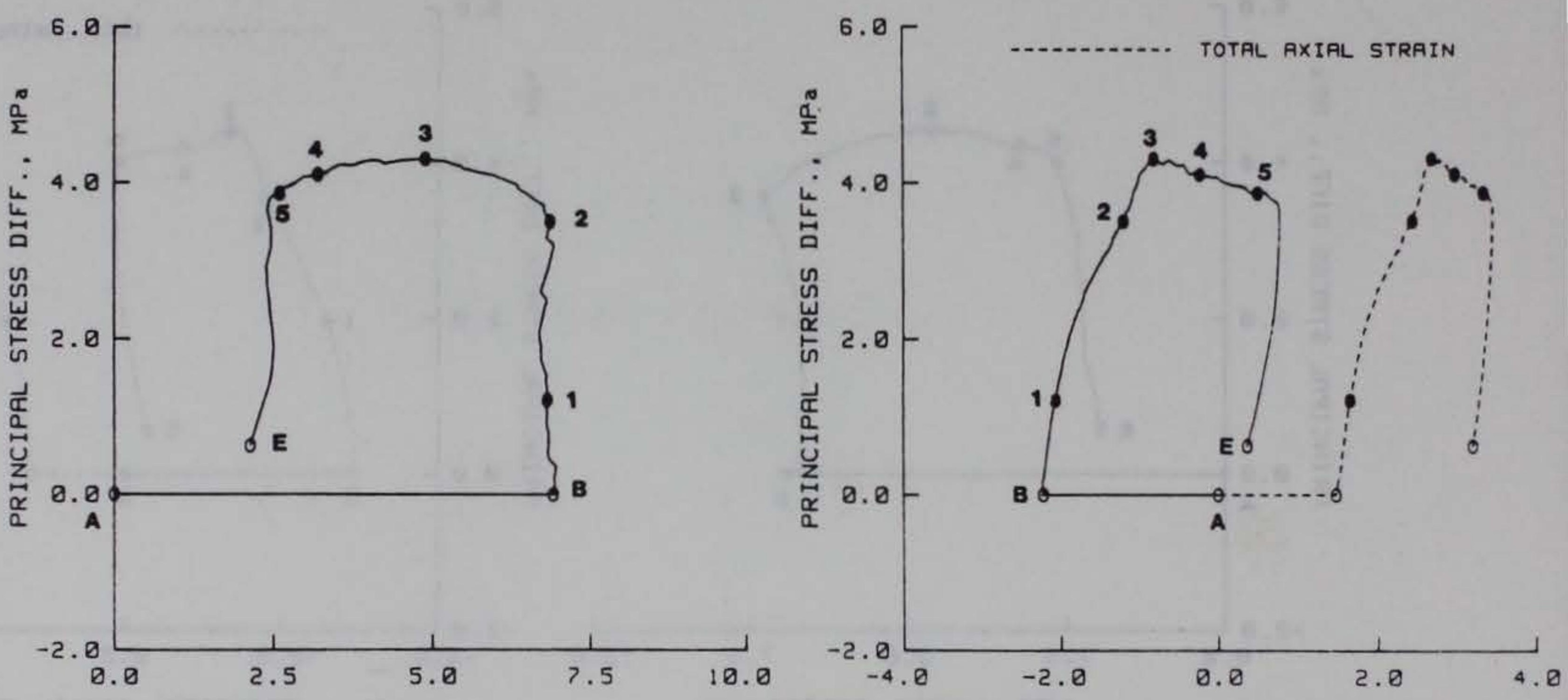
MEAN NORMAL STRESS, MPa
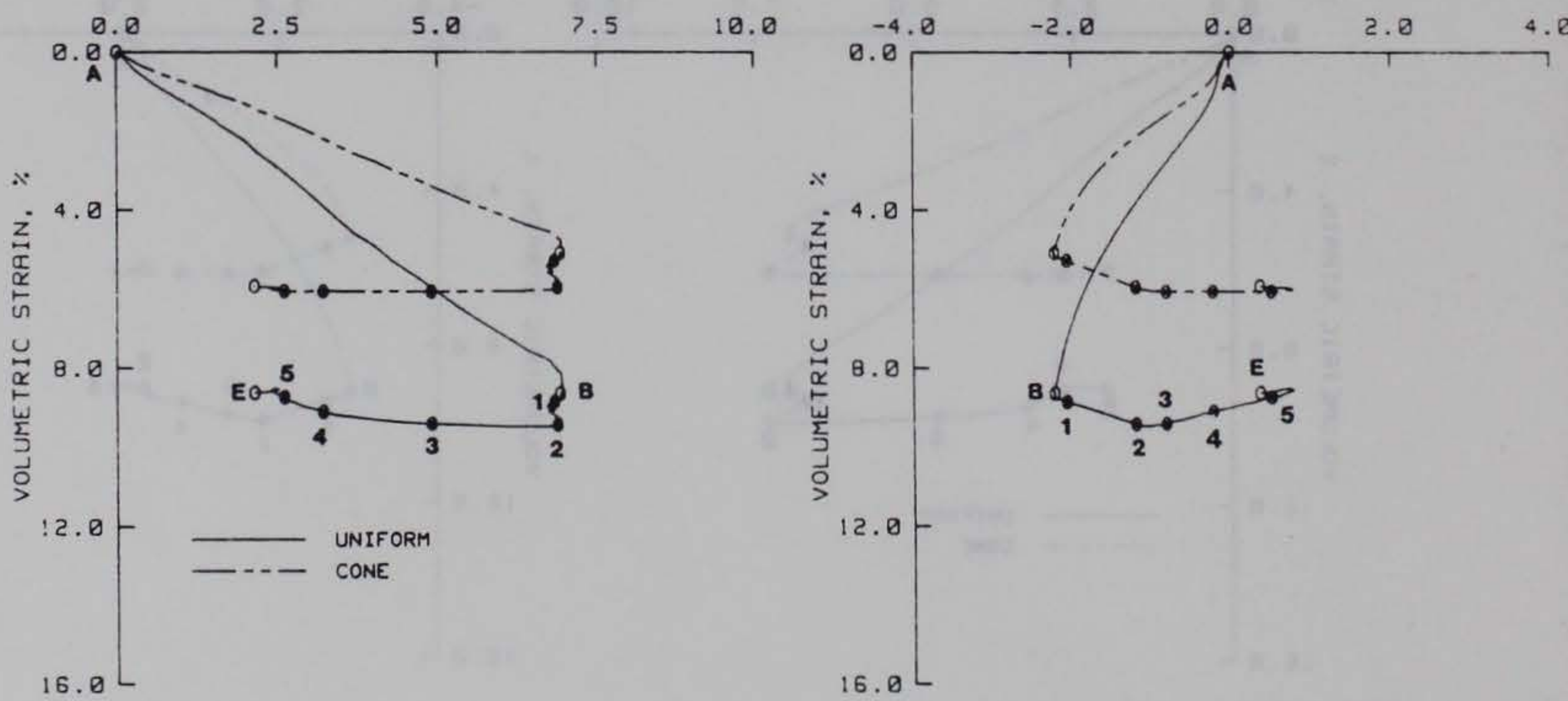


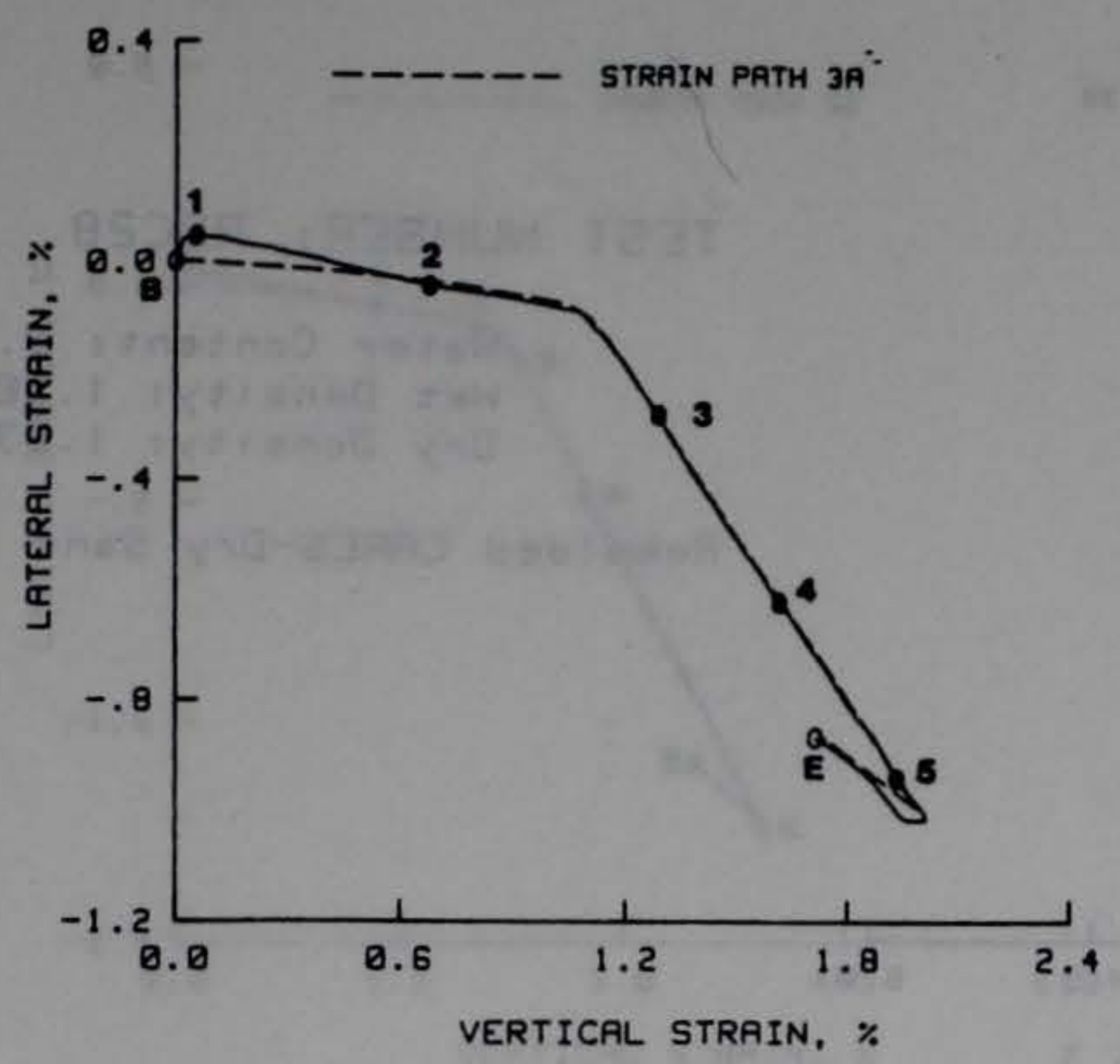

TEST NUMBER: RDC22

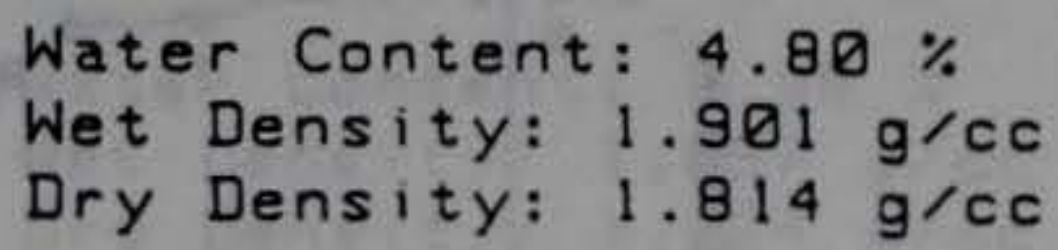

Remolded CARES-Dry Sand
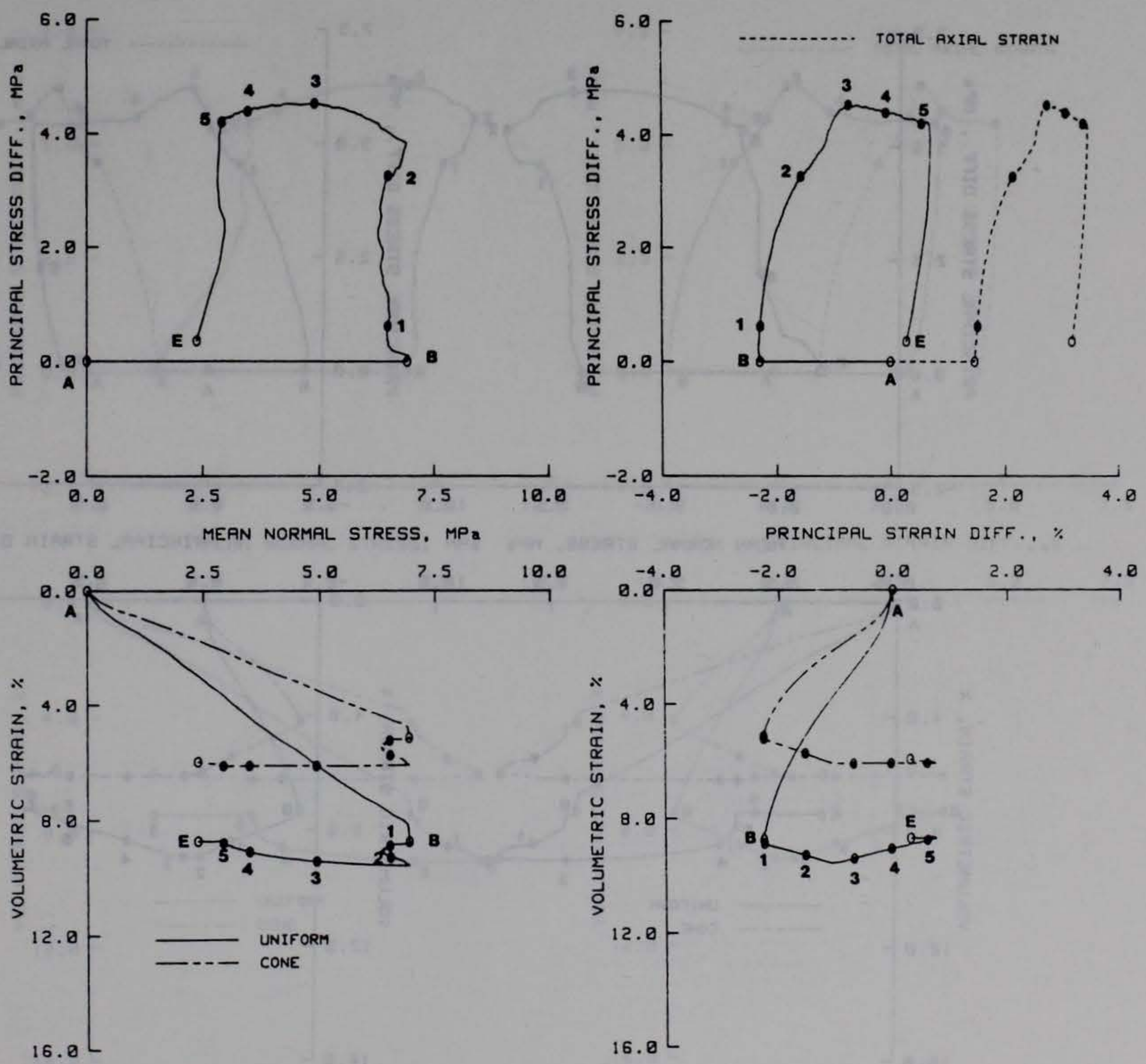


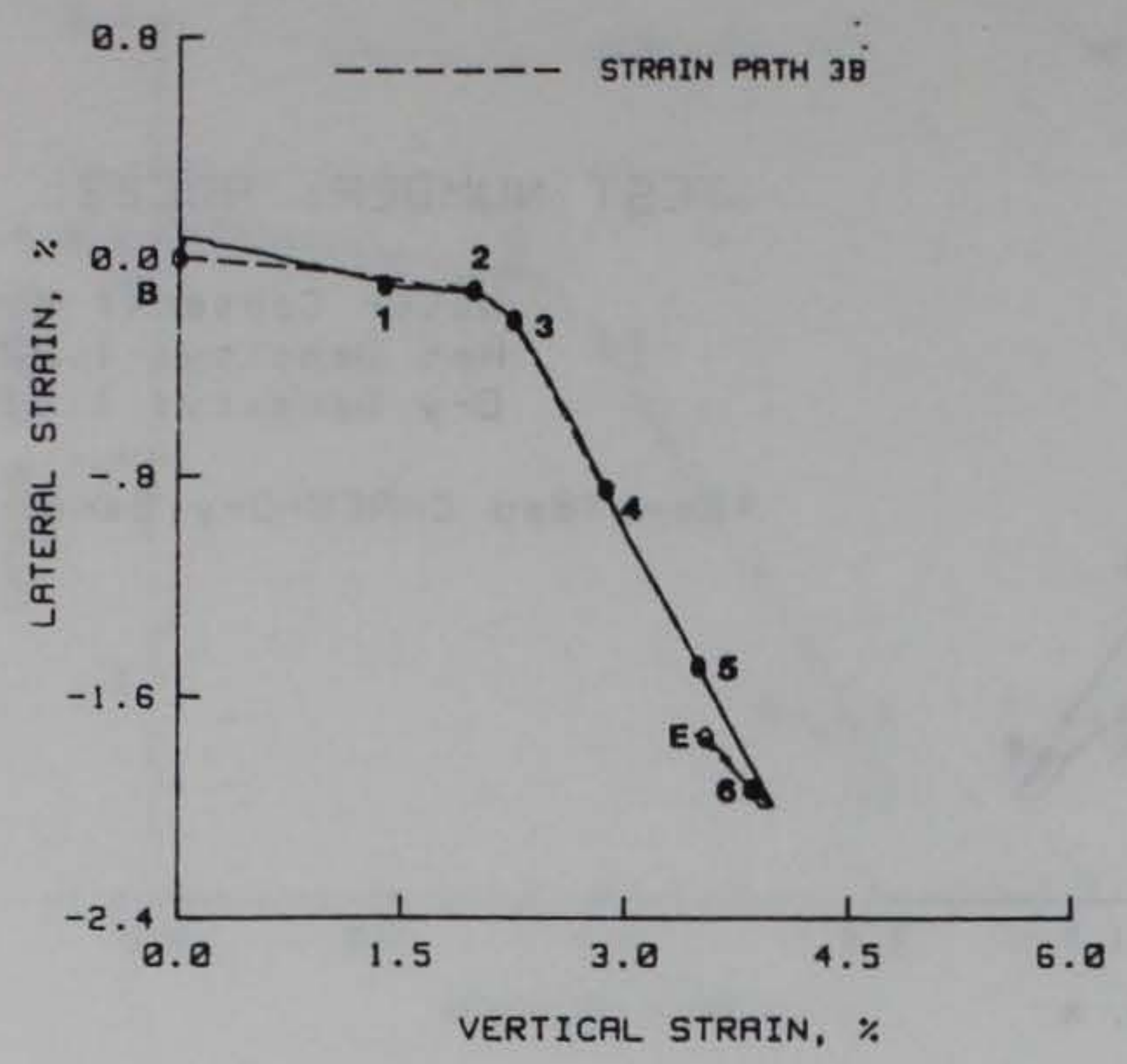

TEST NUMBER: RDC28

Water Content: $3.96 \%$

Wet Density: $1.903 \mathrm{~g} / \mathrm{cc}$

Dry Density: $1.831 \mathrm{~g} / \mathrm{cc}$

Remolded CARES-Dry Sand
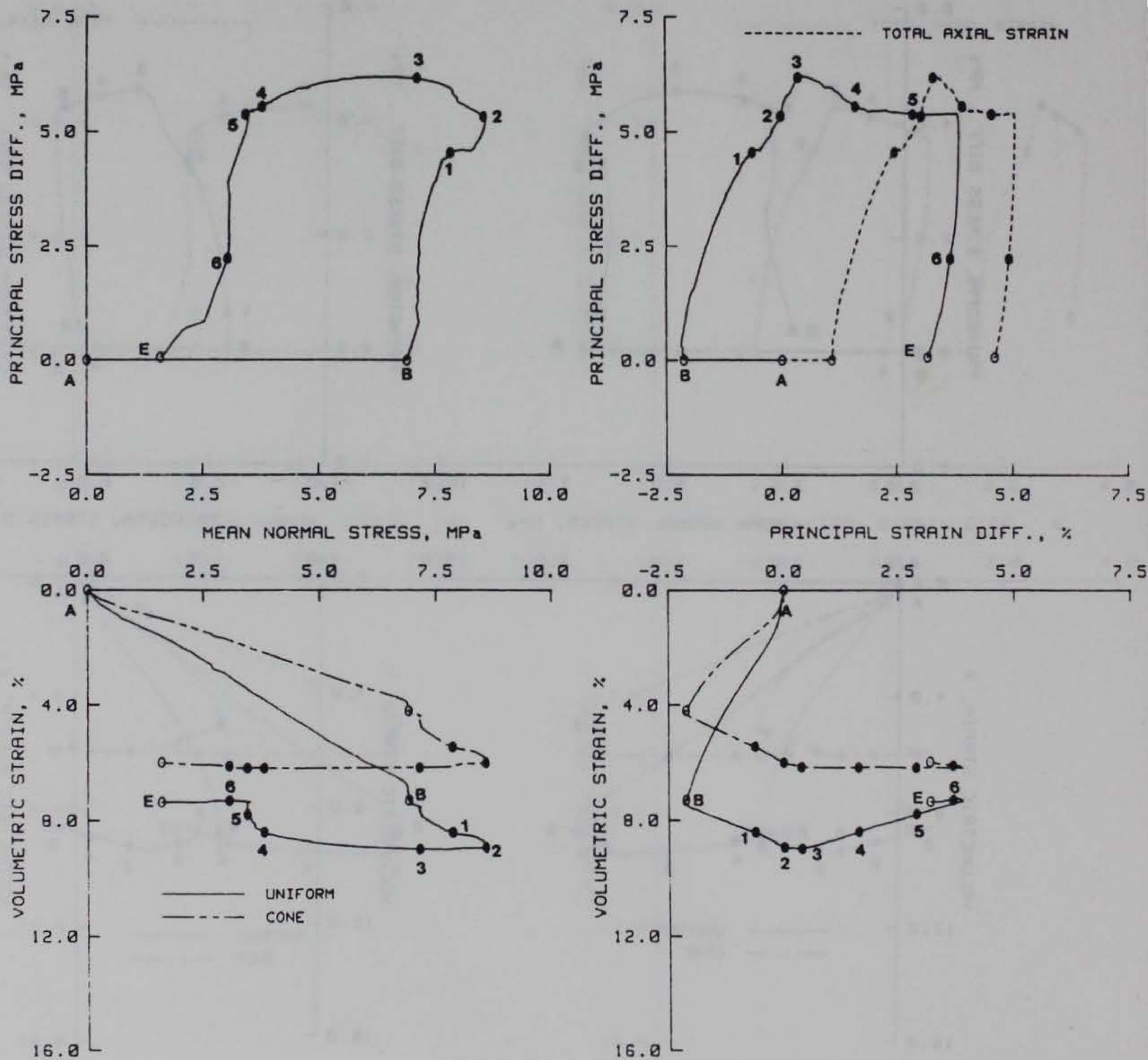


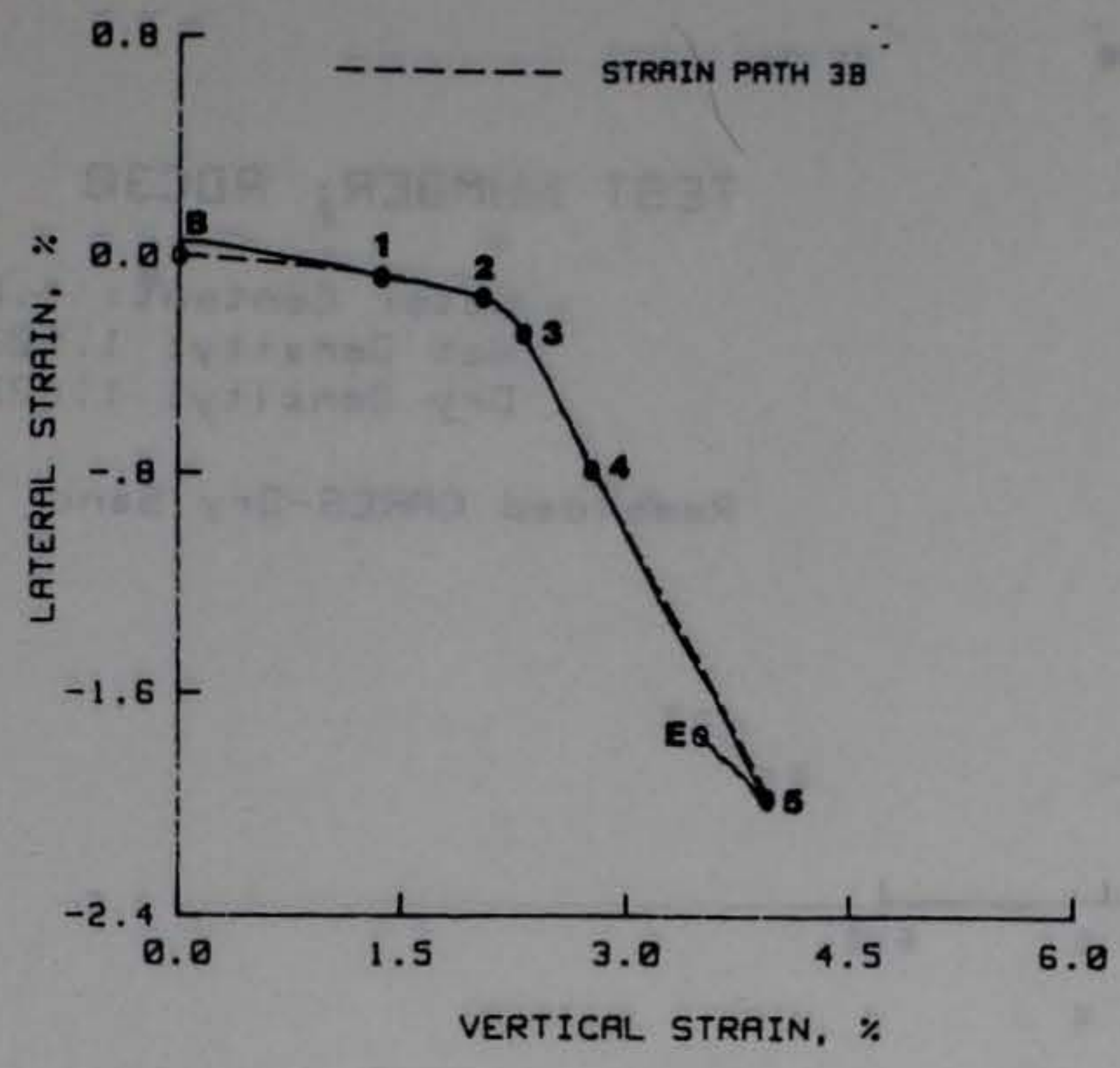

TEST NUMBER: RDC29

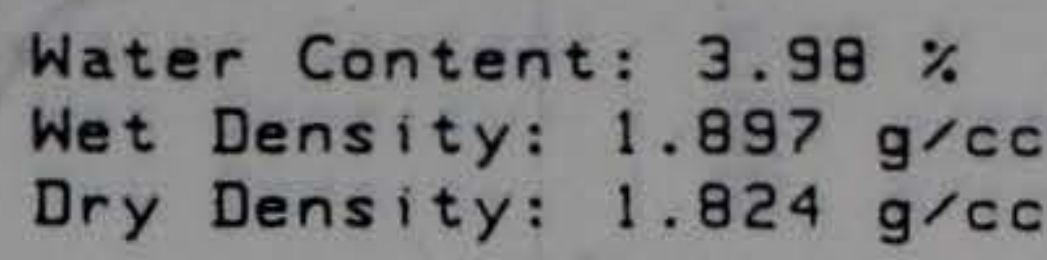

Remolded CARES-Dry Sand
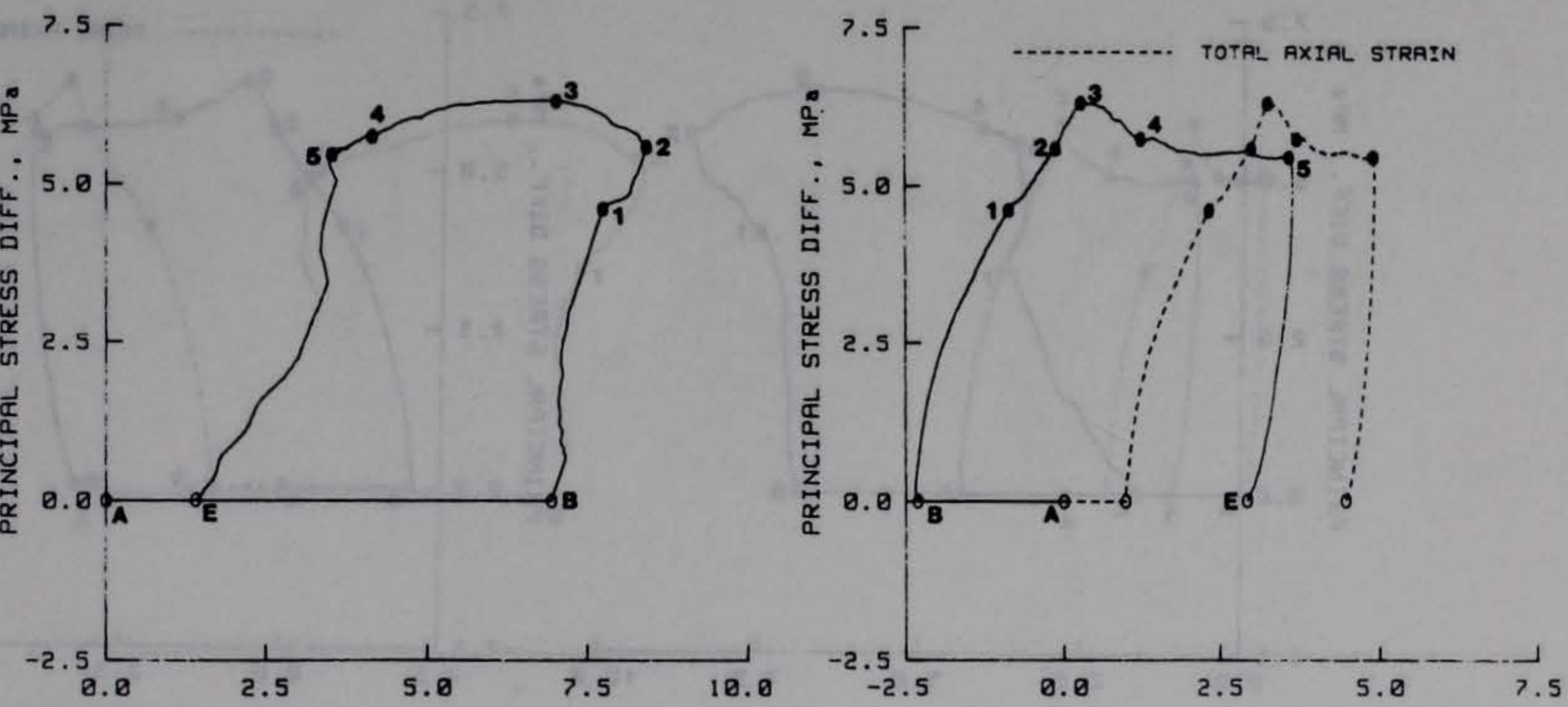

MEAN NORMAL STRESS, MPa
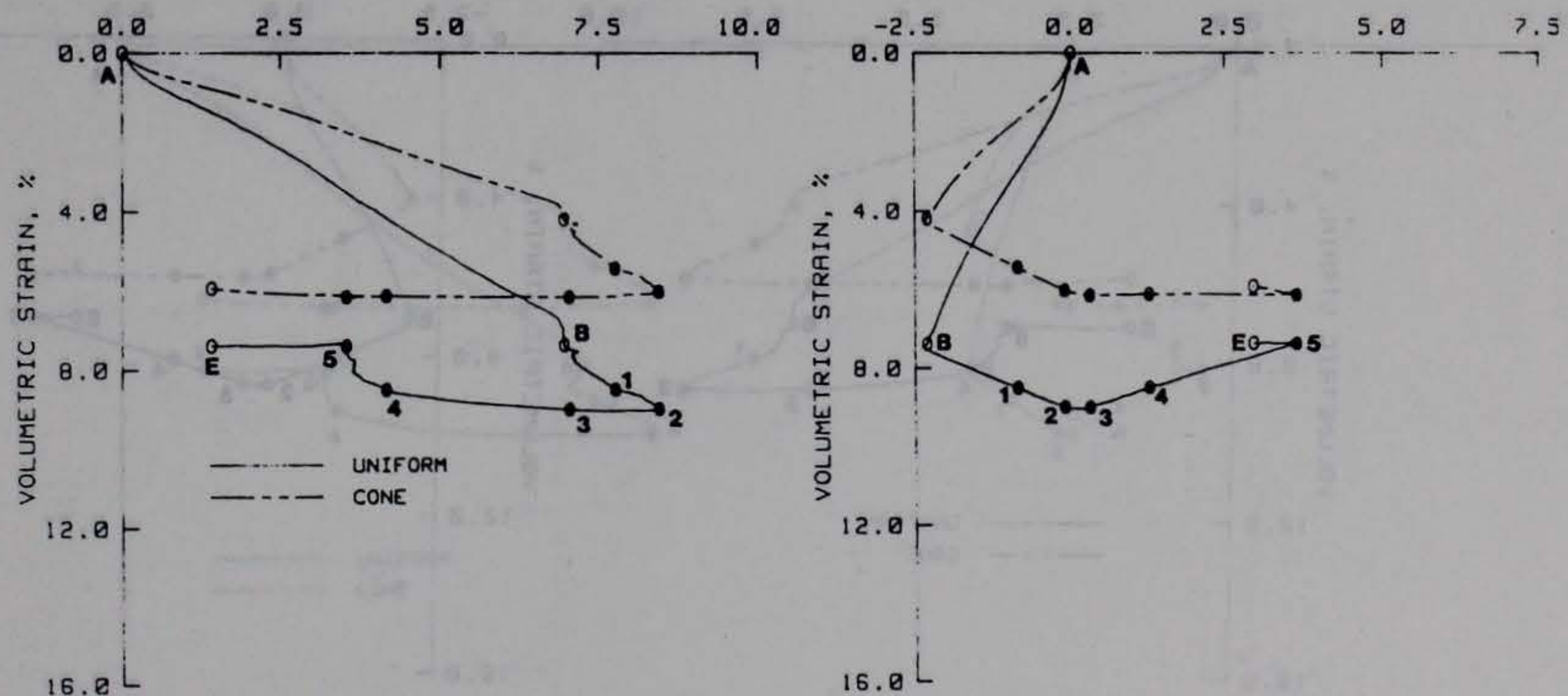

12.05

$16.0 \mathrm{~L}$ 


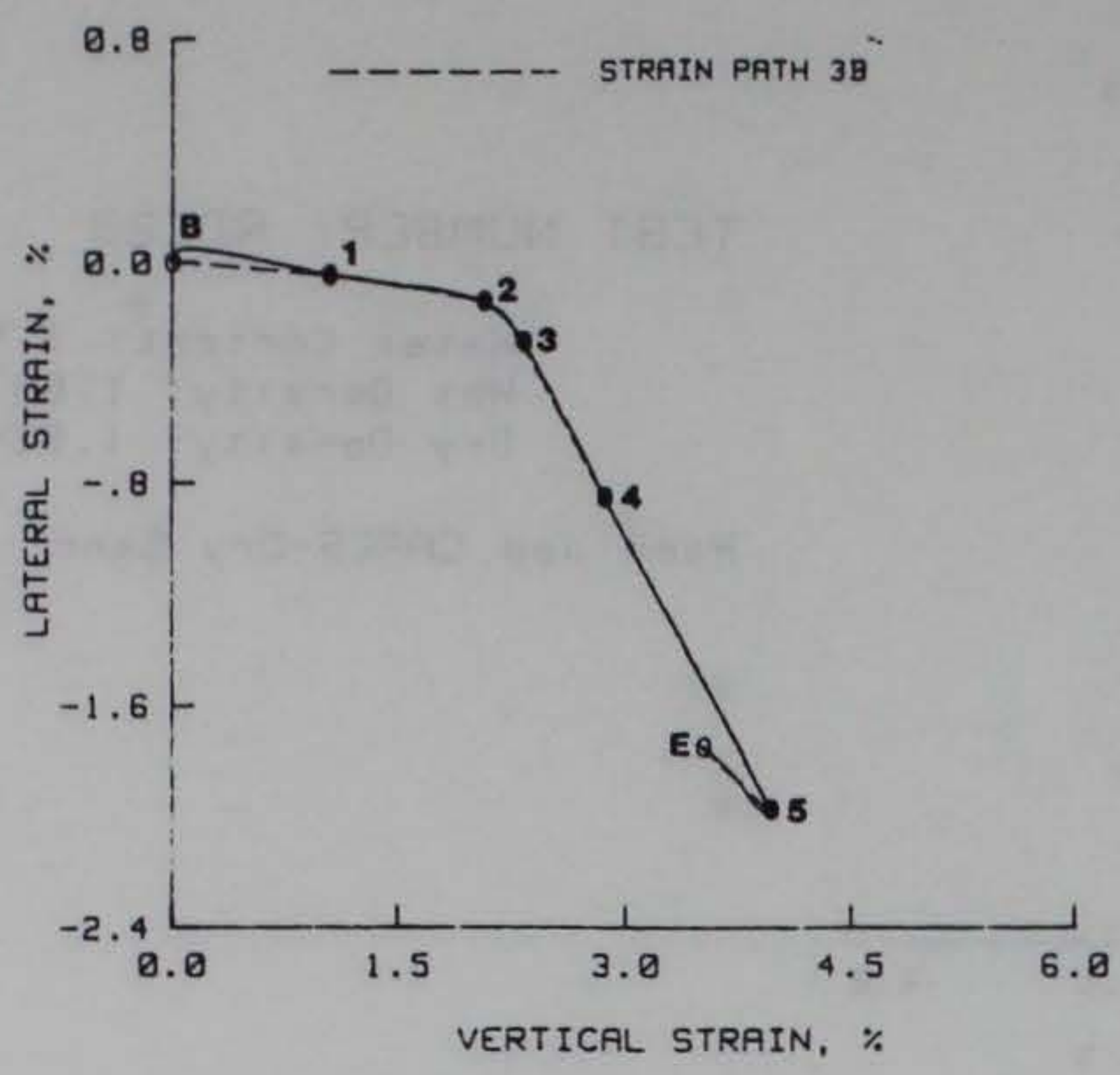

TEST NUMBER: RDC30

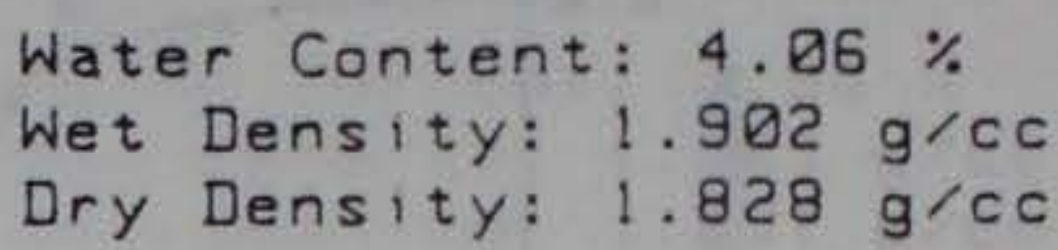

Remolded CARES-Dry Sand
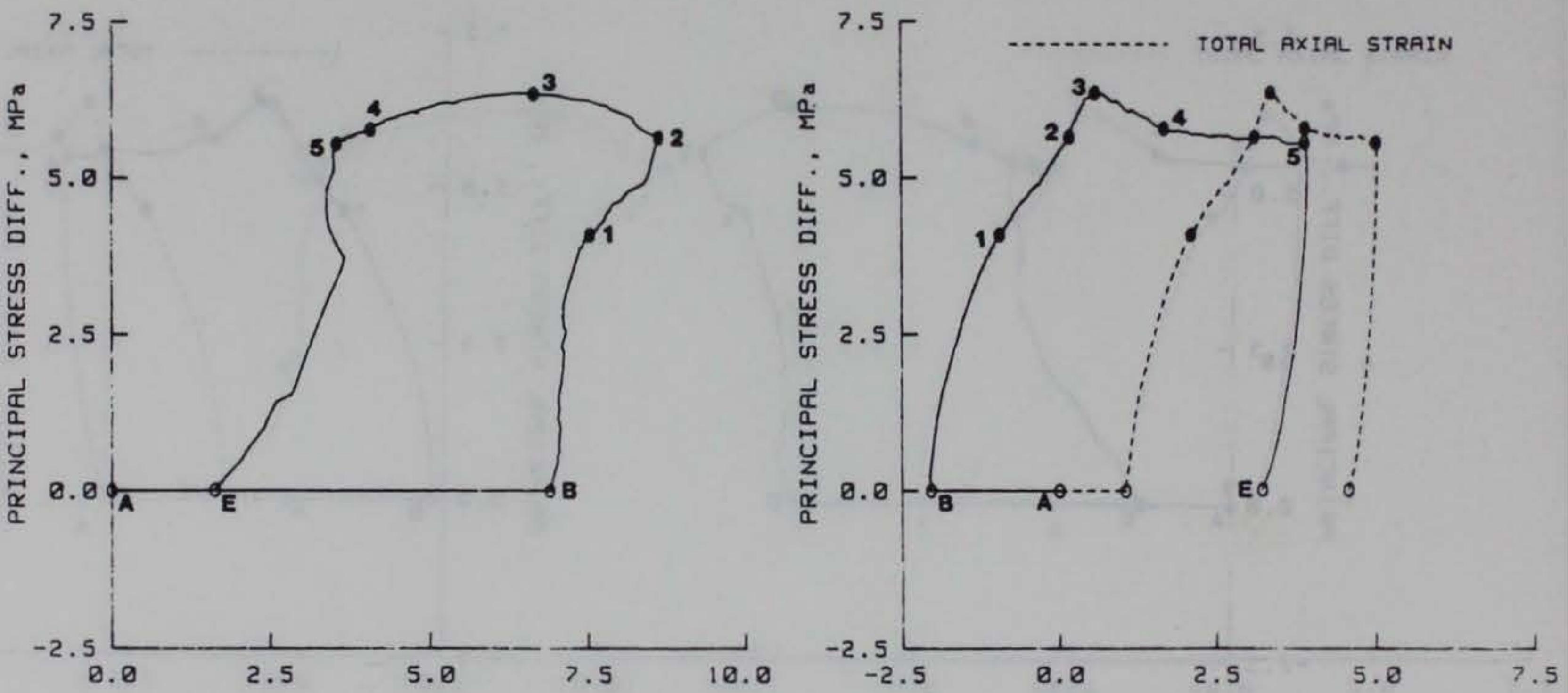

MEAN NORMAL STRESS, MPa
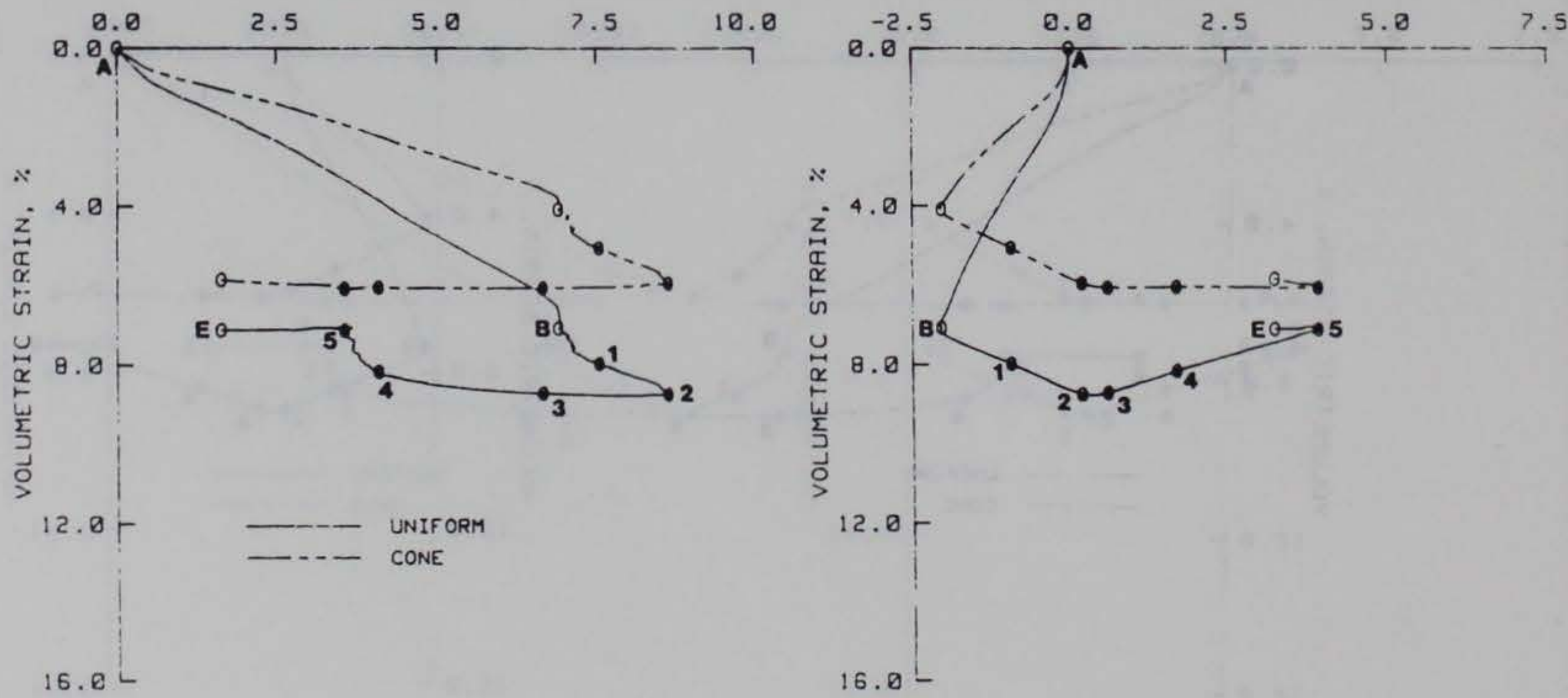


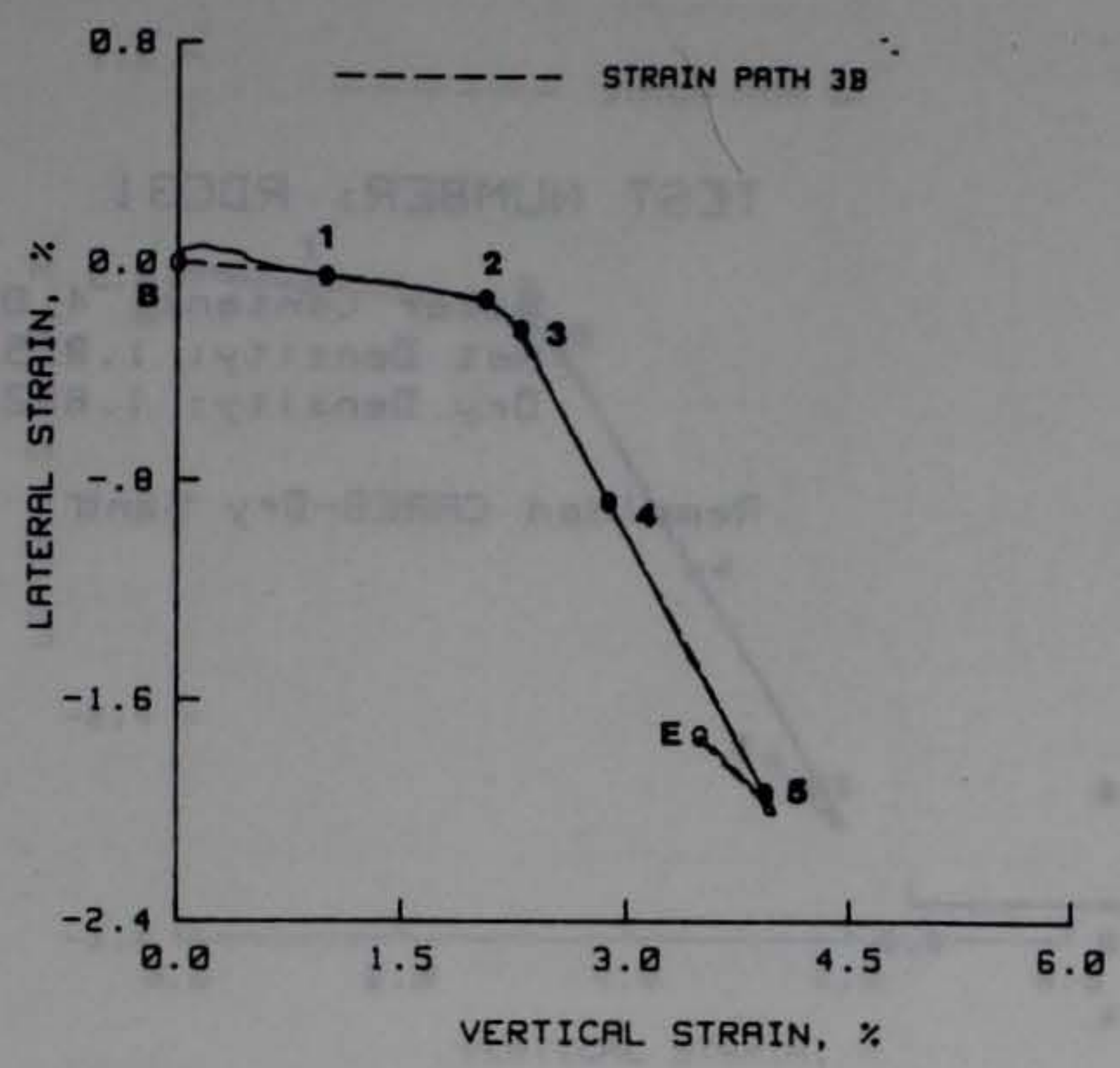

TEST NUMBER: RDC62

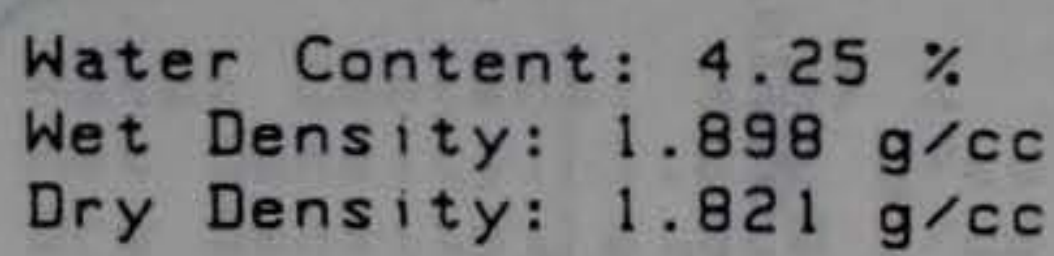

Remolded CARES-Dry Sand

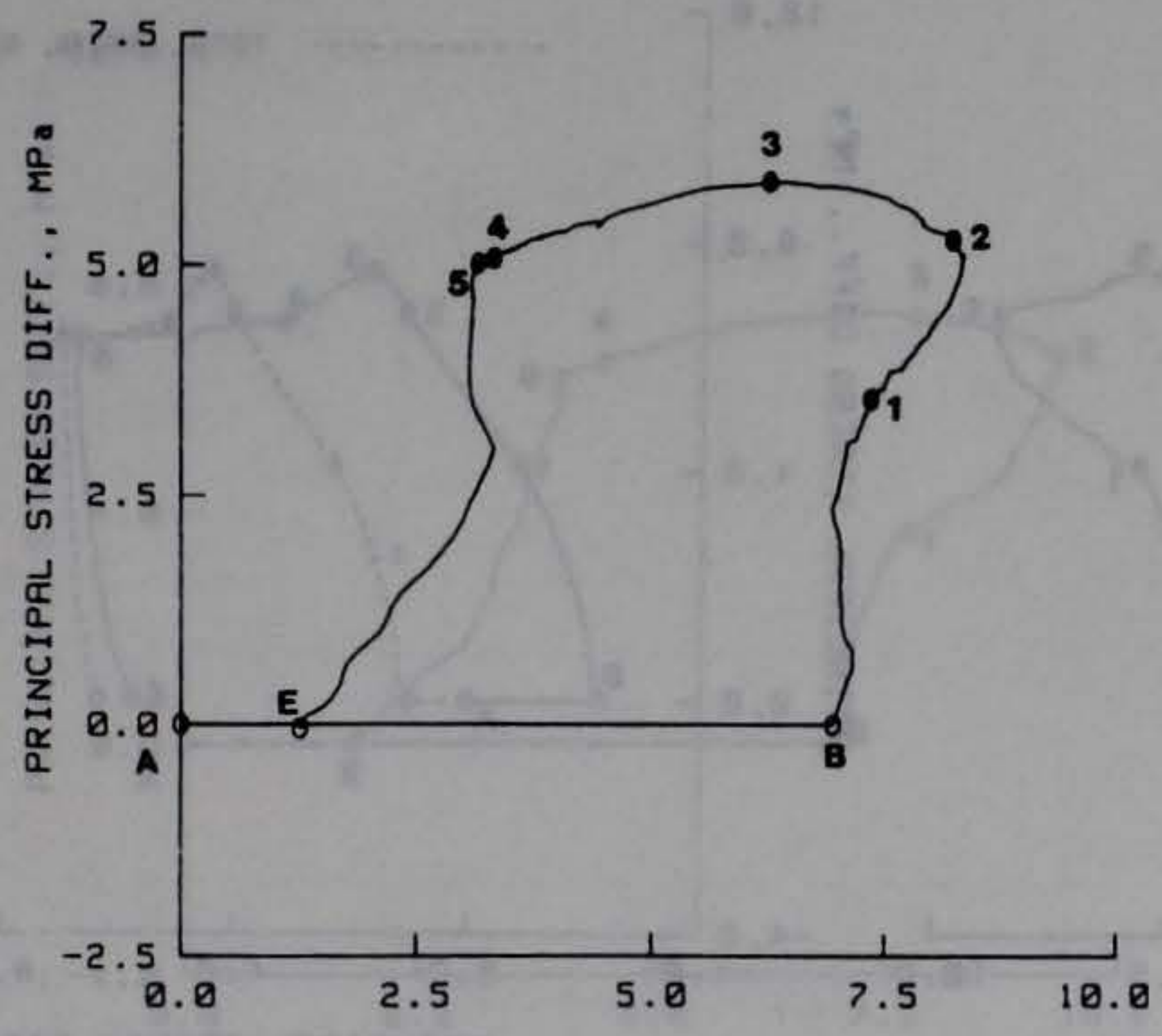

MEAN NORMAL STRESS, MPa

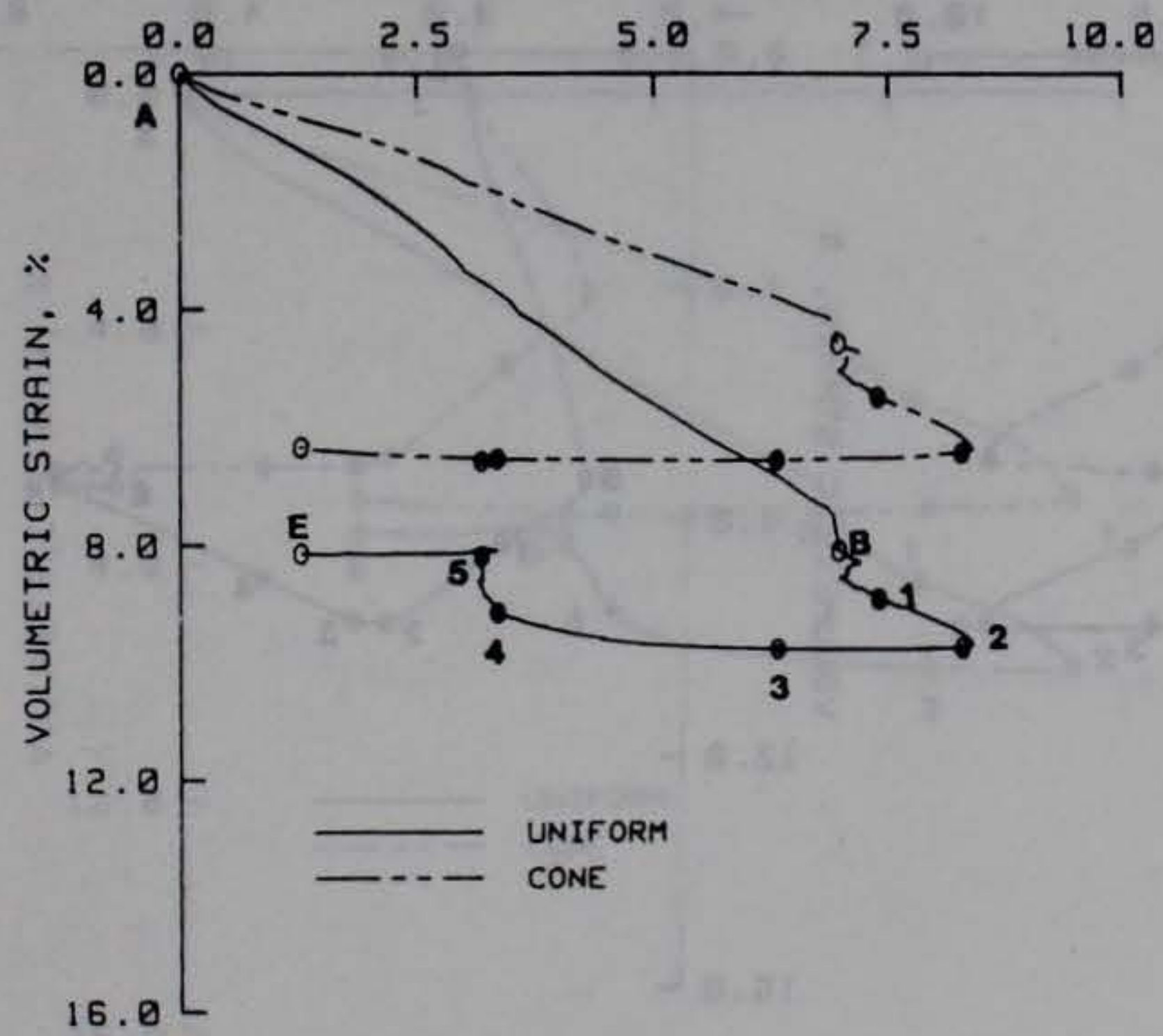

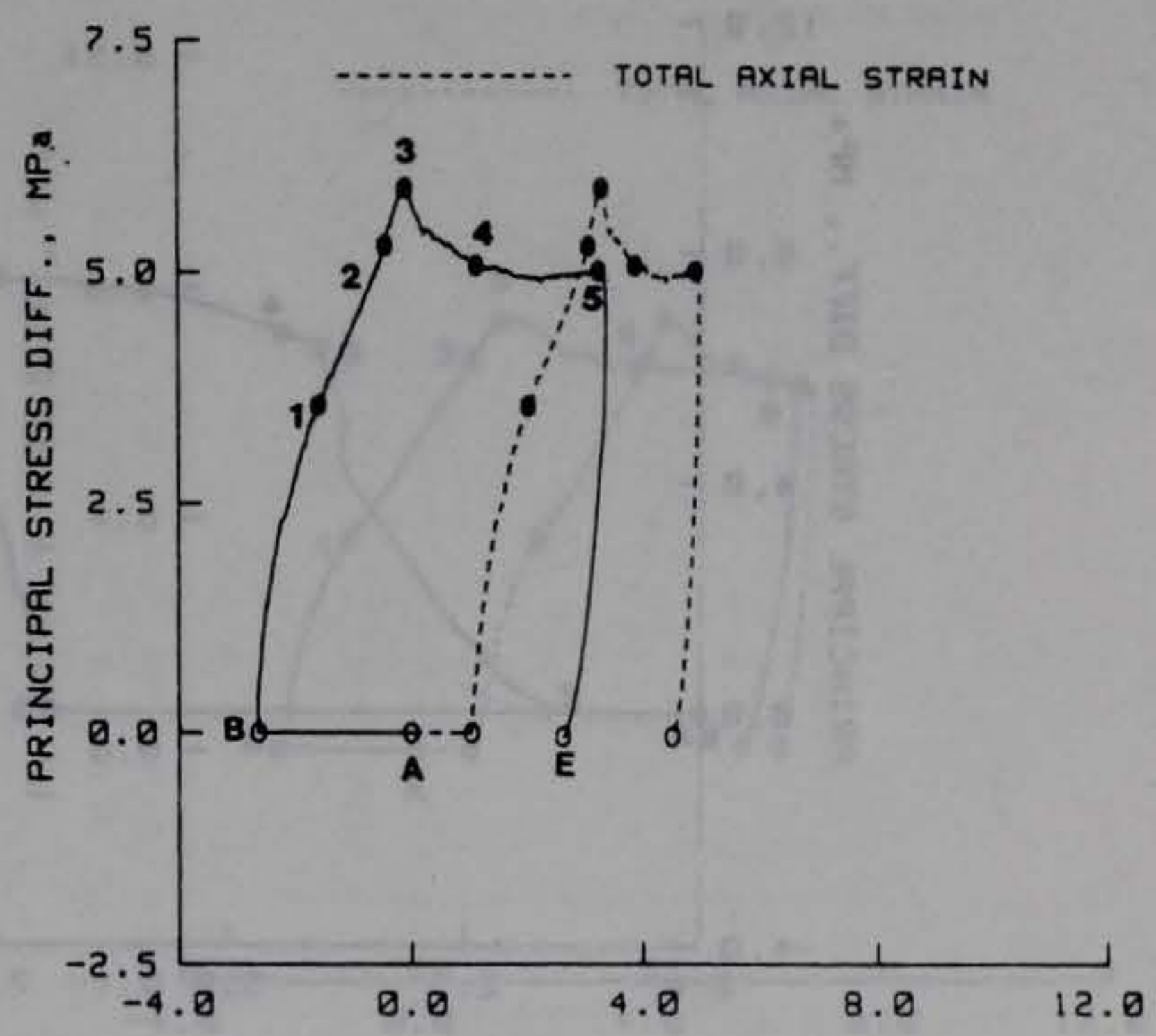

PRINCIPAL STRAIN DIFF., \%

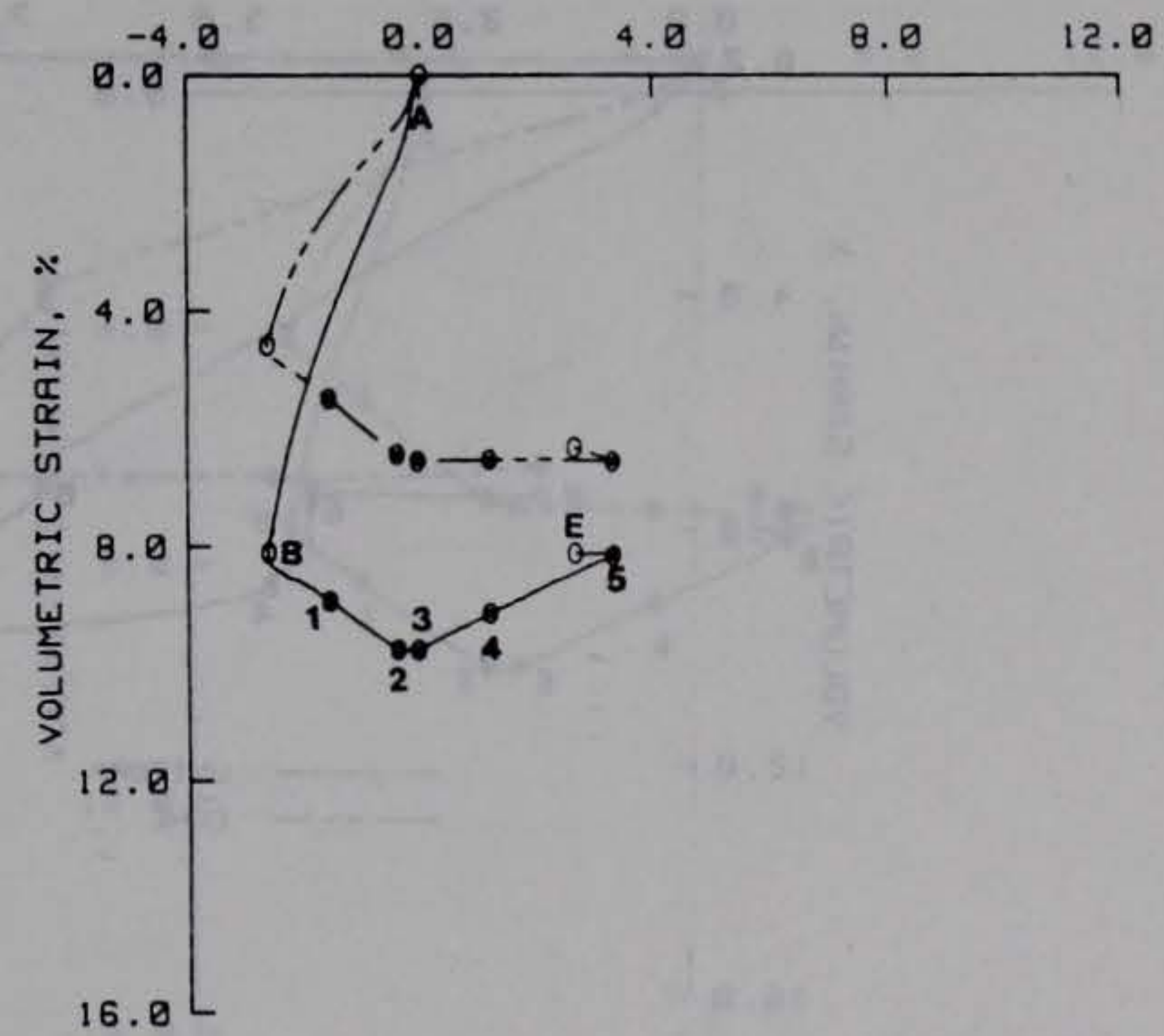




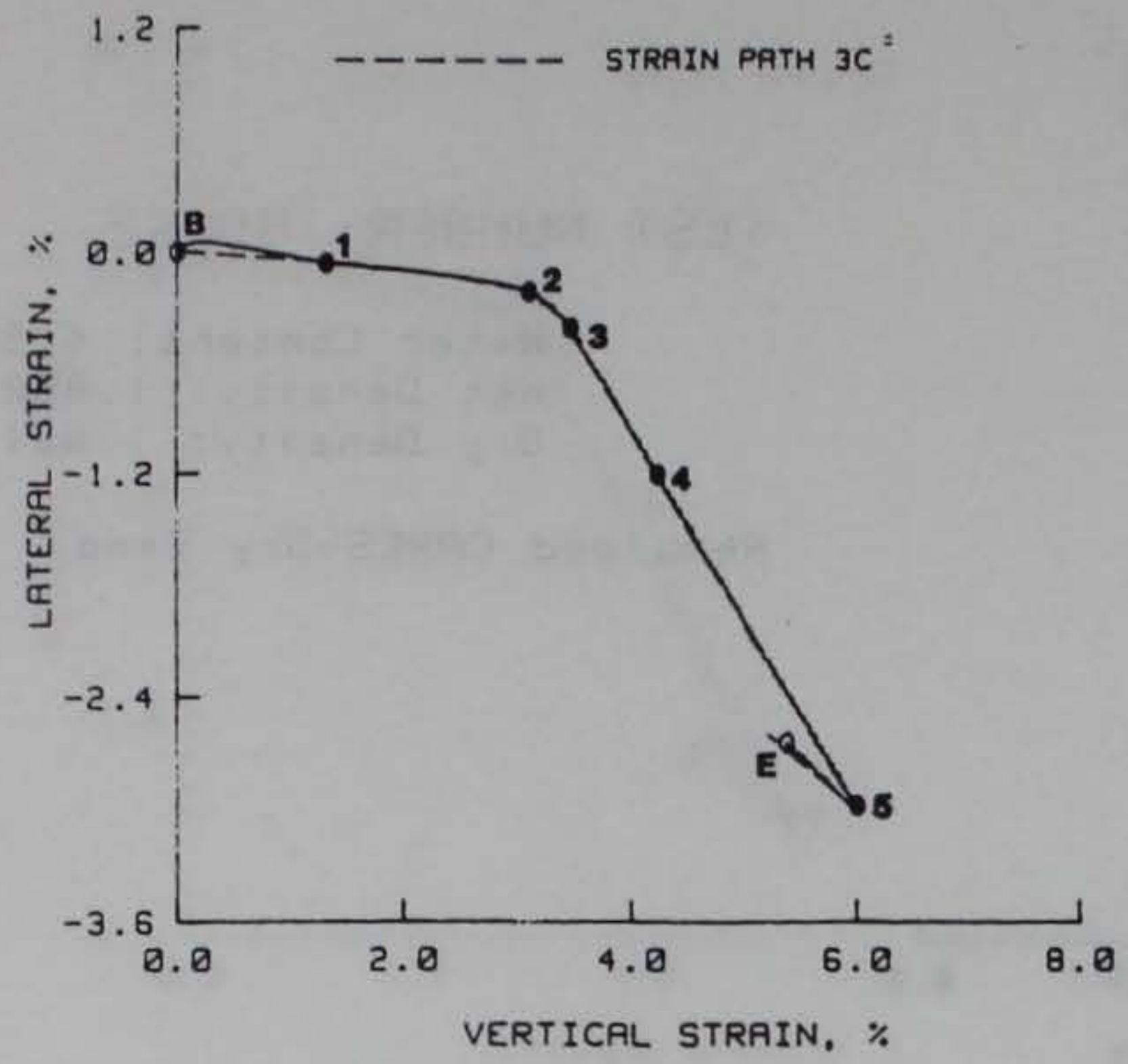

TEST NUMBER: RDC31

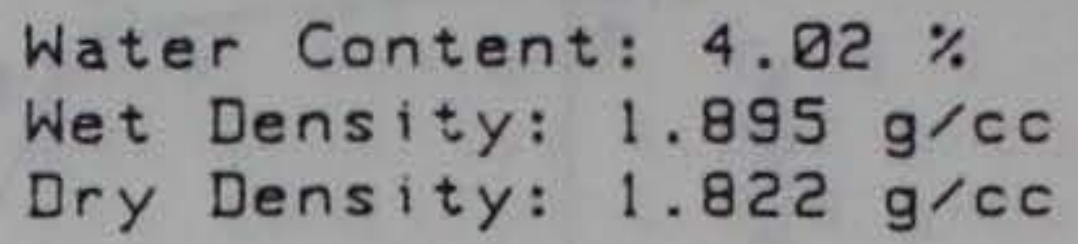

Remolded CARES-Dry Sand
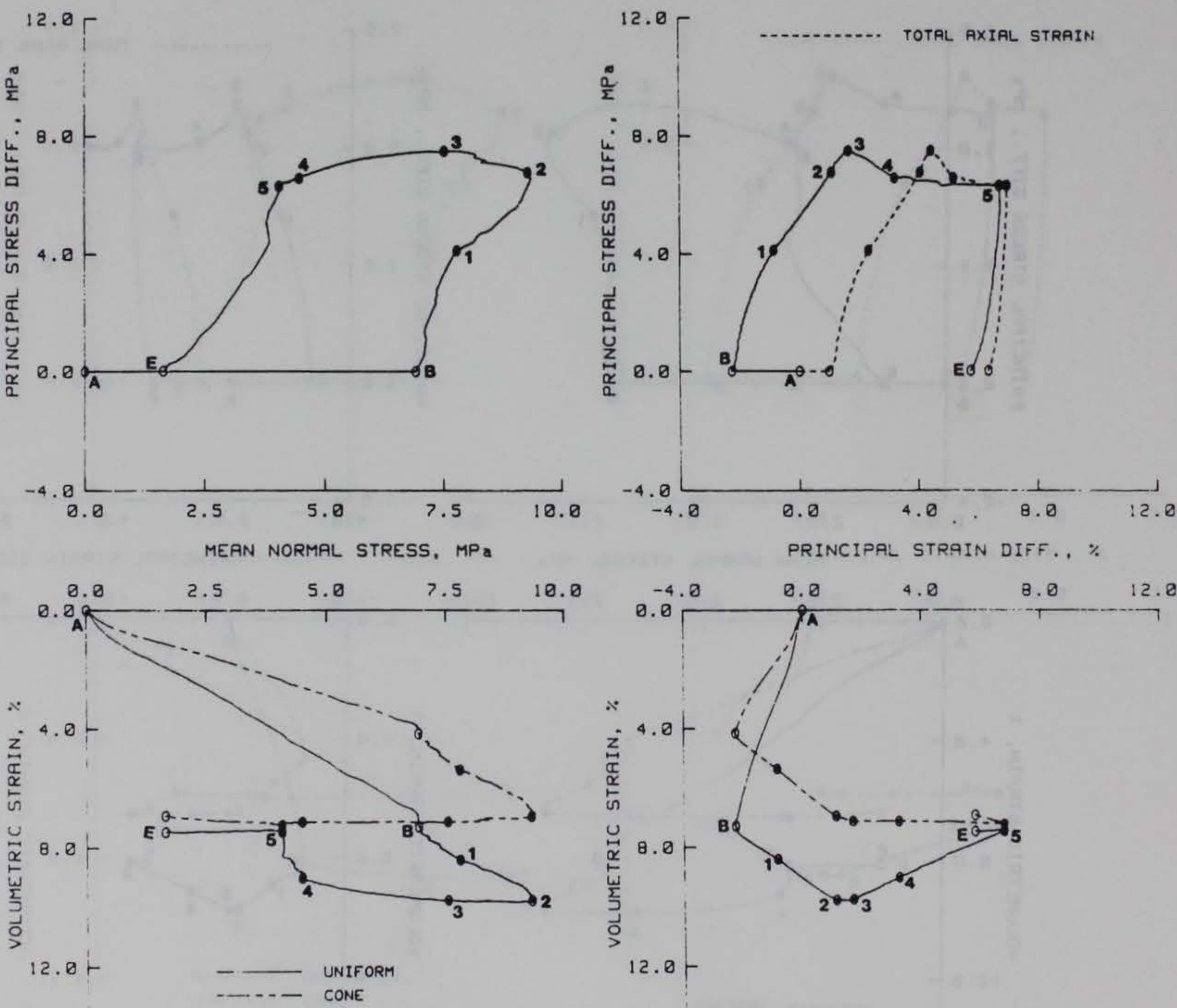


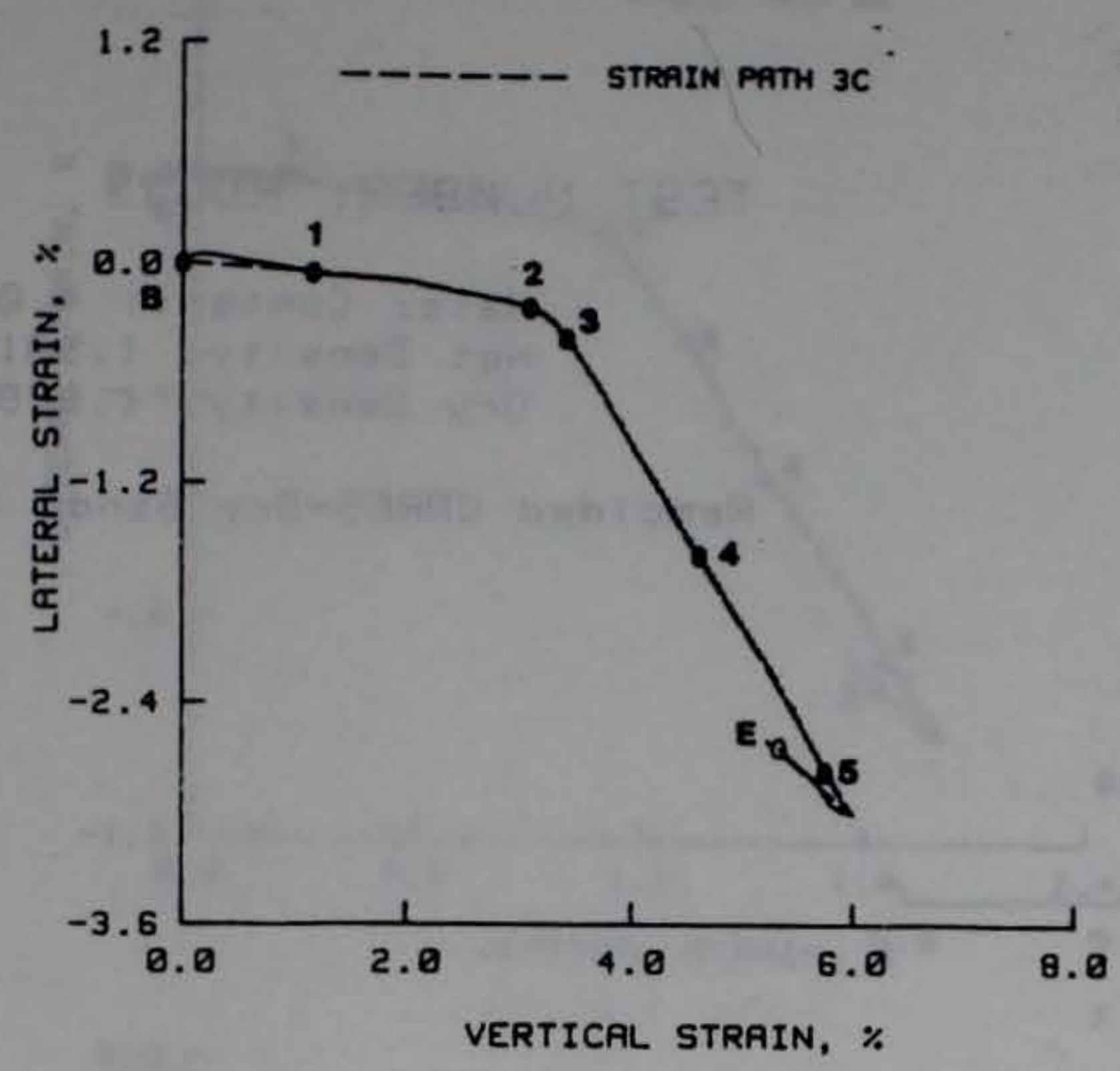

TEST NUMBER: RDC32

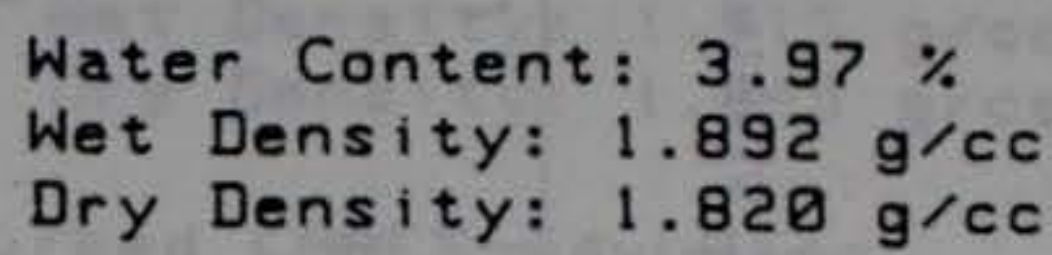

Remolded CARES-Dry Sand
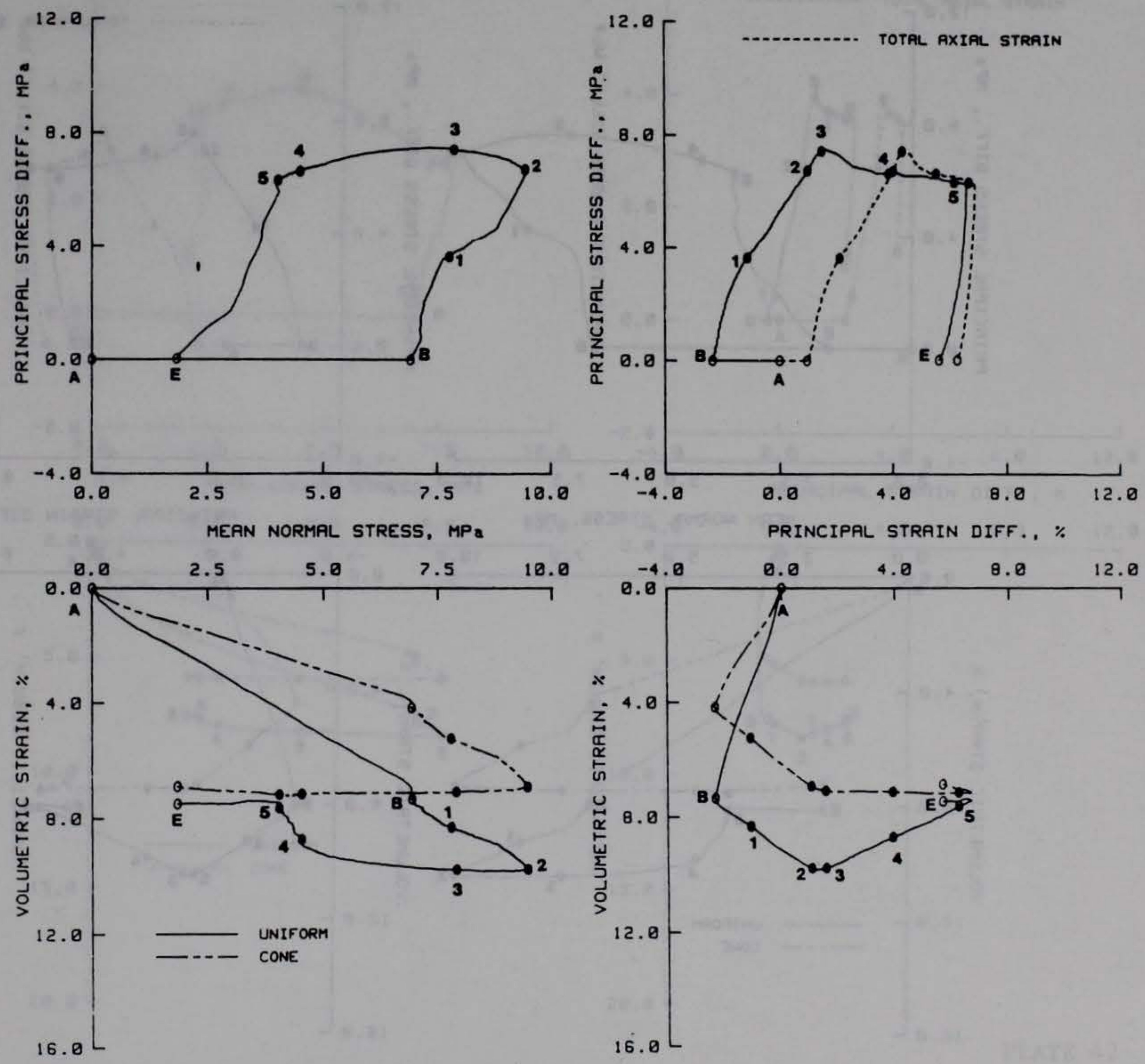


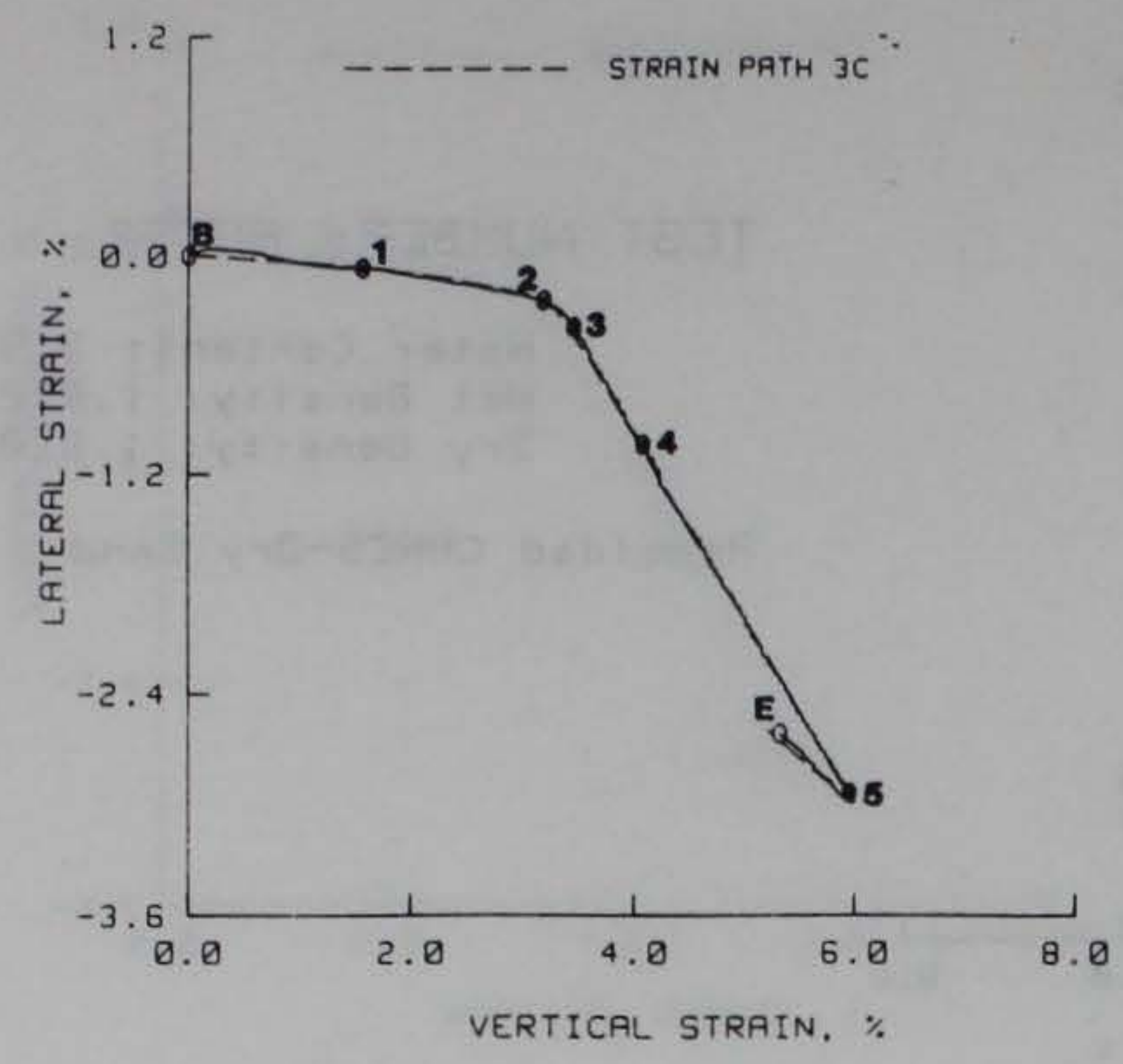

TEST NUMBER: RDC33

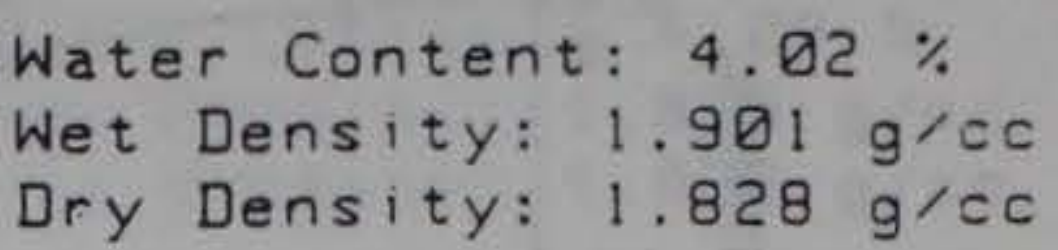

Remolded CARES-Dry Sand
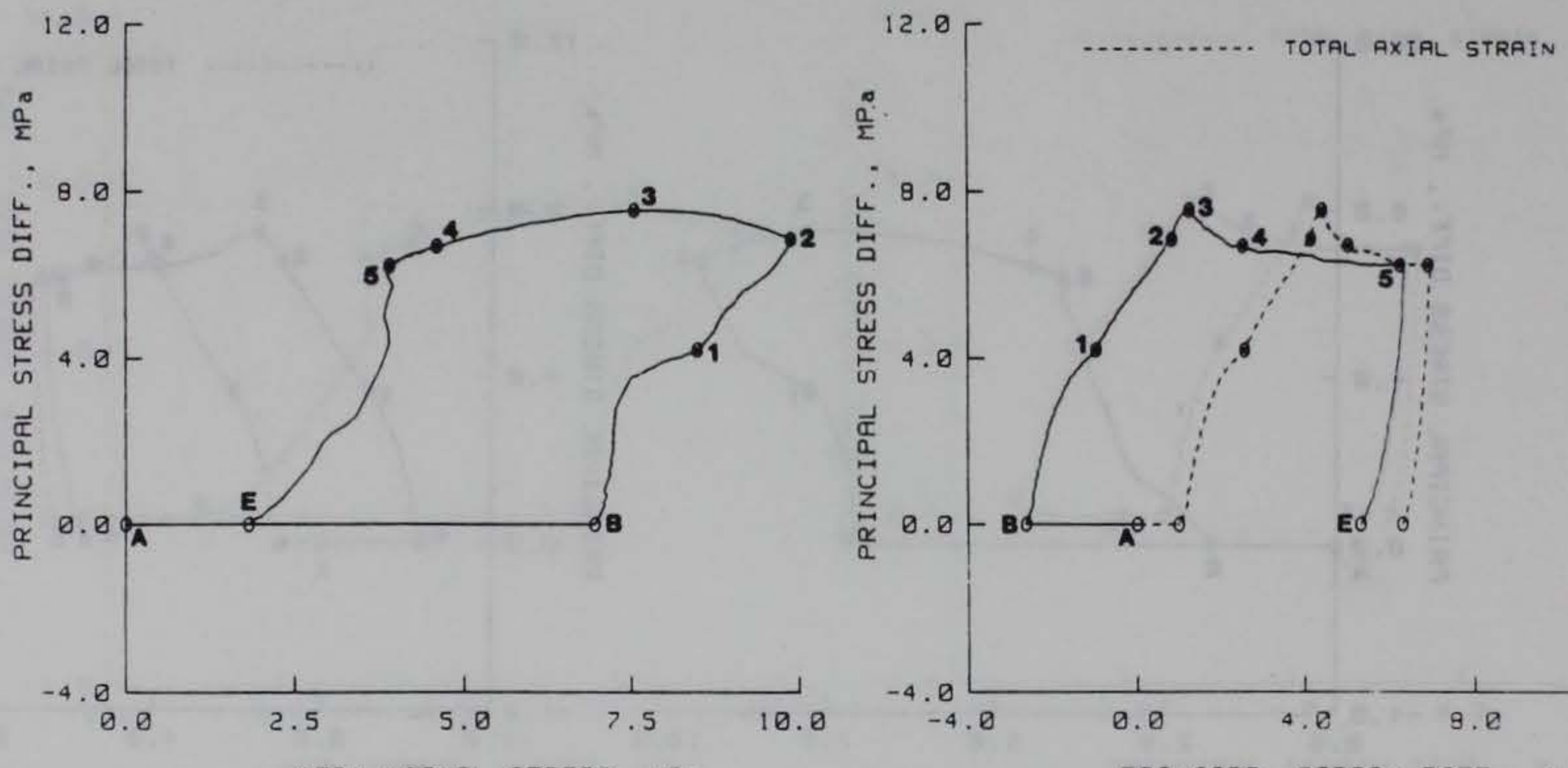
MEAN NORMAL STRESS, MPa
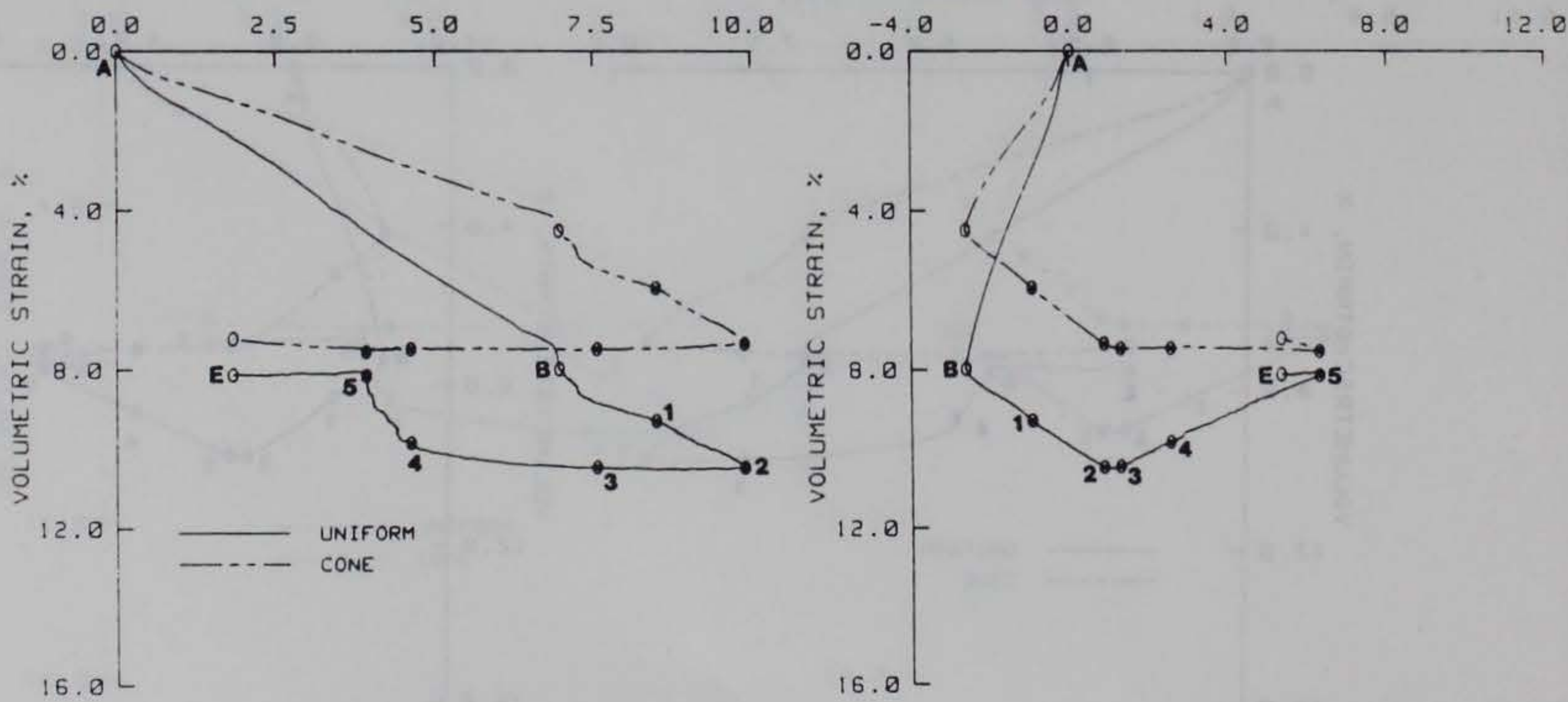


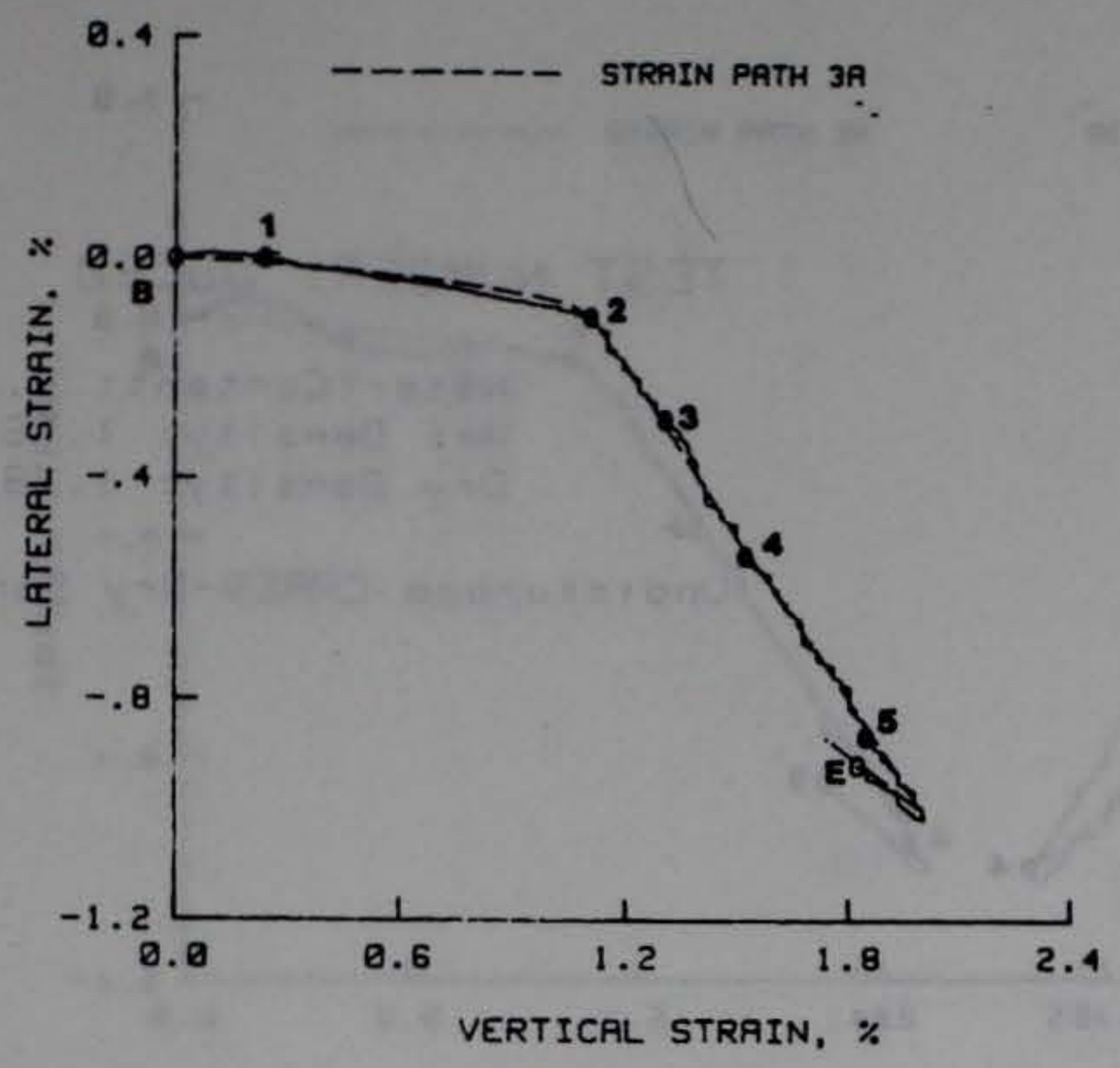

TEST NUMBER: UDC59

Water Content: $3.80 \%$ Wet Density: $1.915 \mathrm{~g} / \mathrm{cc}$ Dry Density: $1.845 \mathrm{~g} / \mathrm{cc}$

Und isturbed CARES-Dry Sand

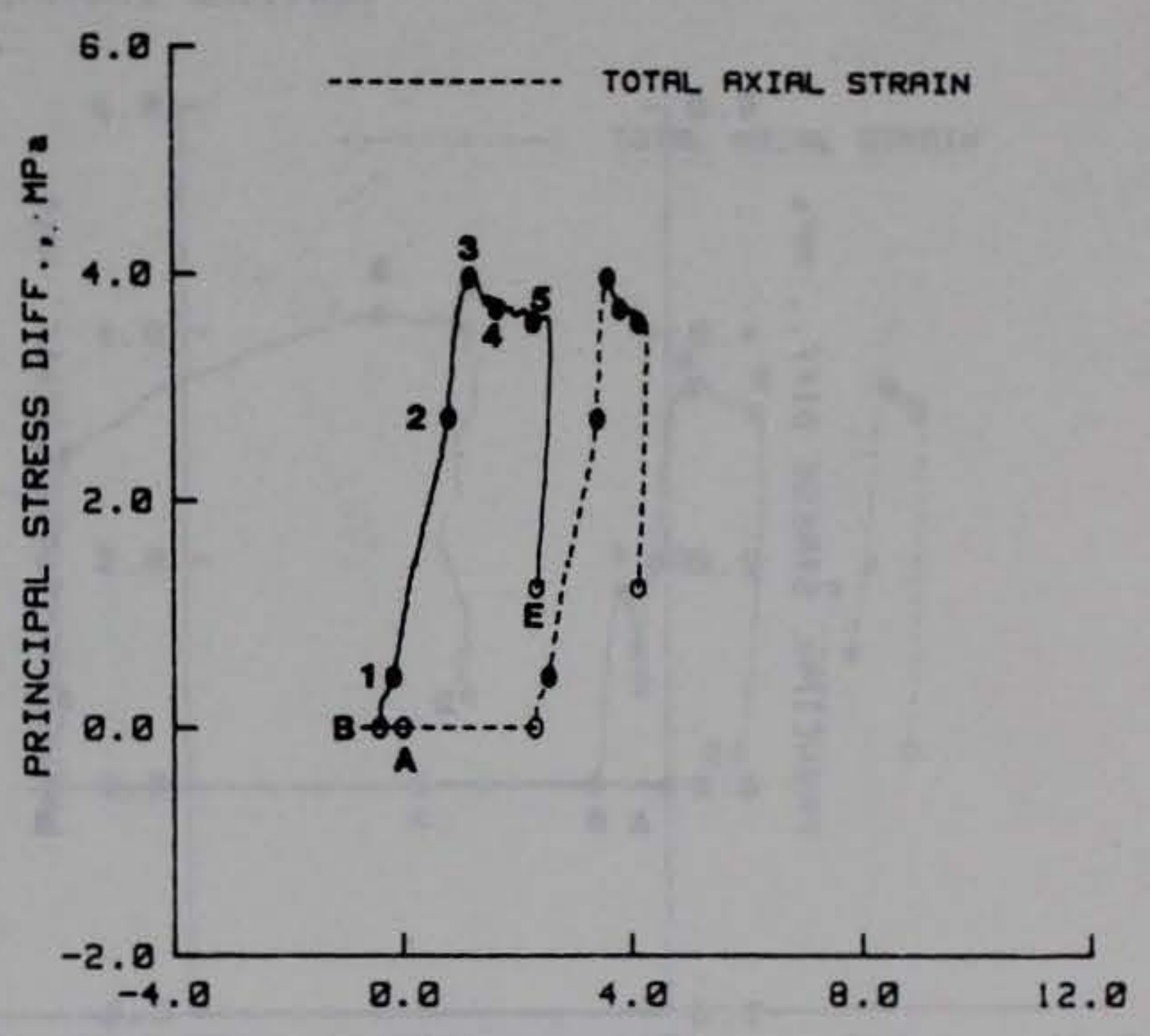
PRINCIPAL STRAIN DIFF., \%

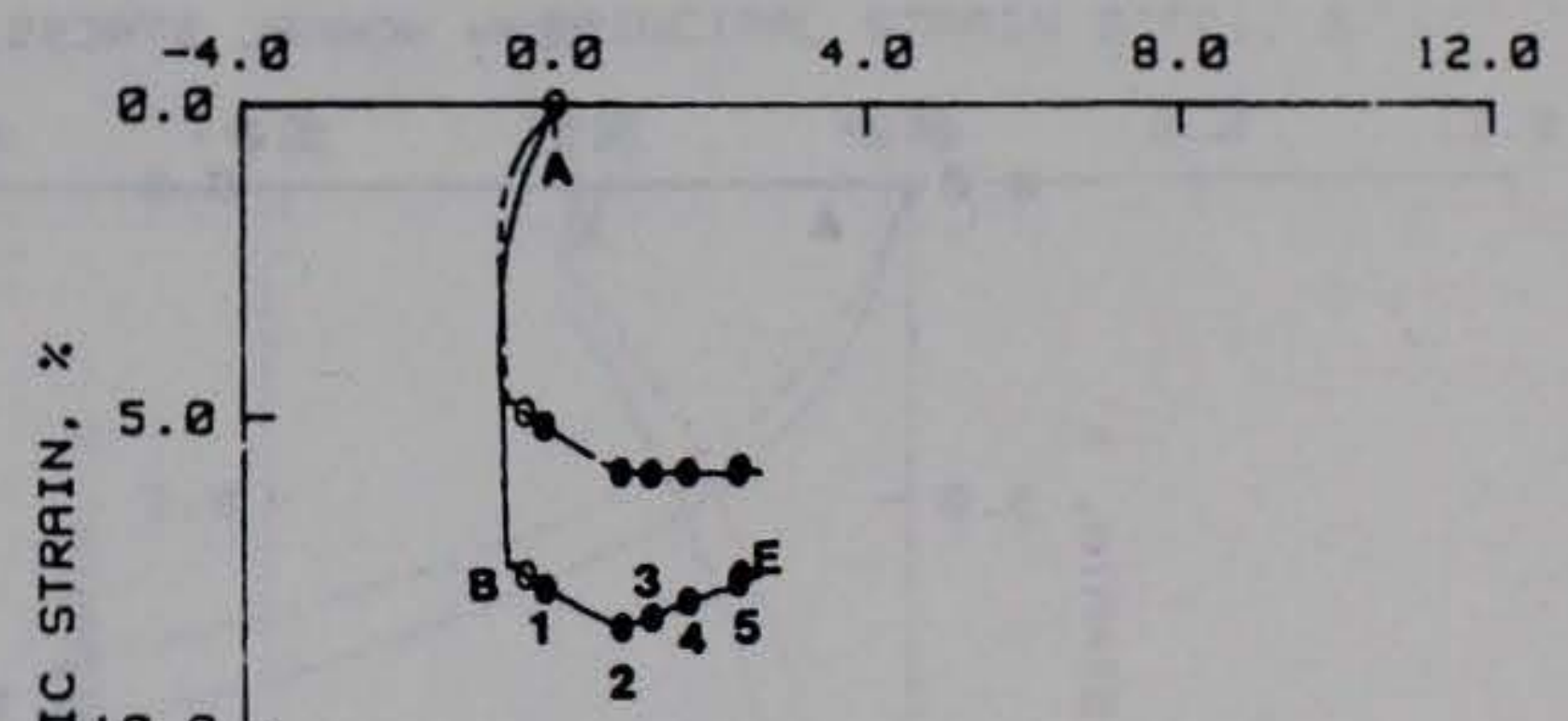

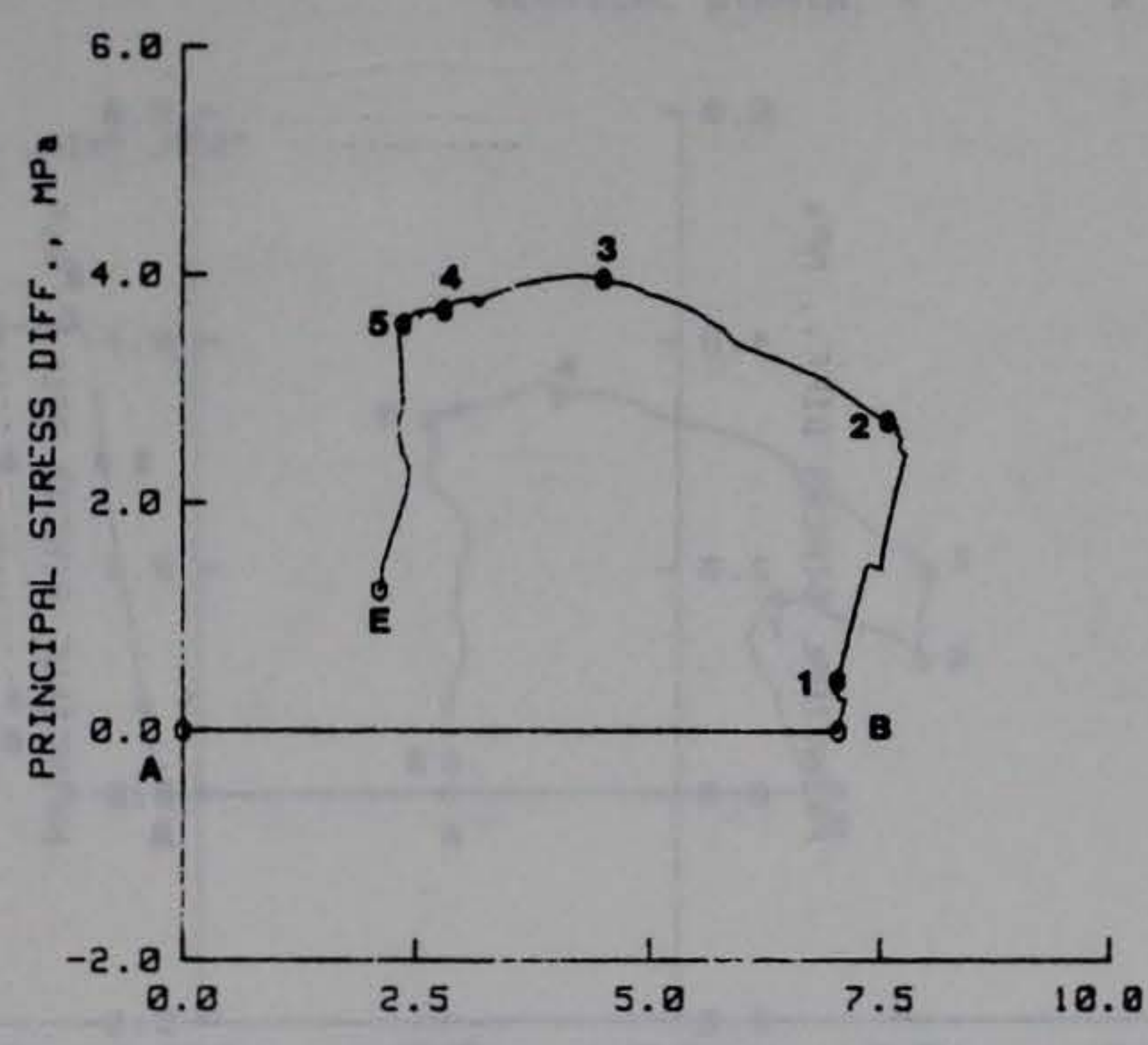
MEAN NORMAL STRESS, MPa

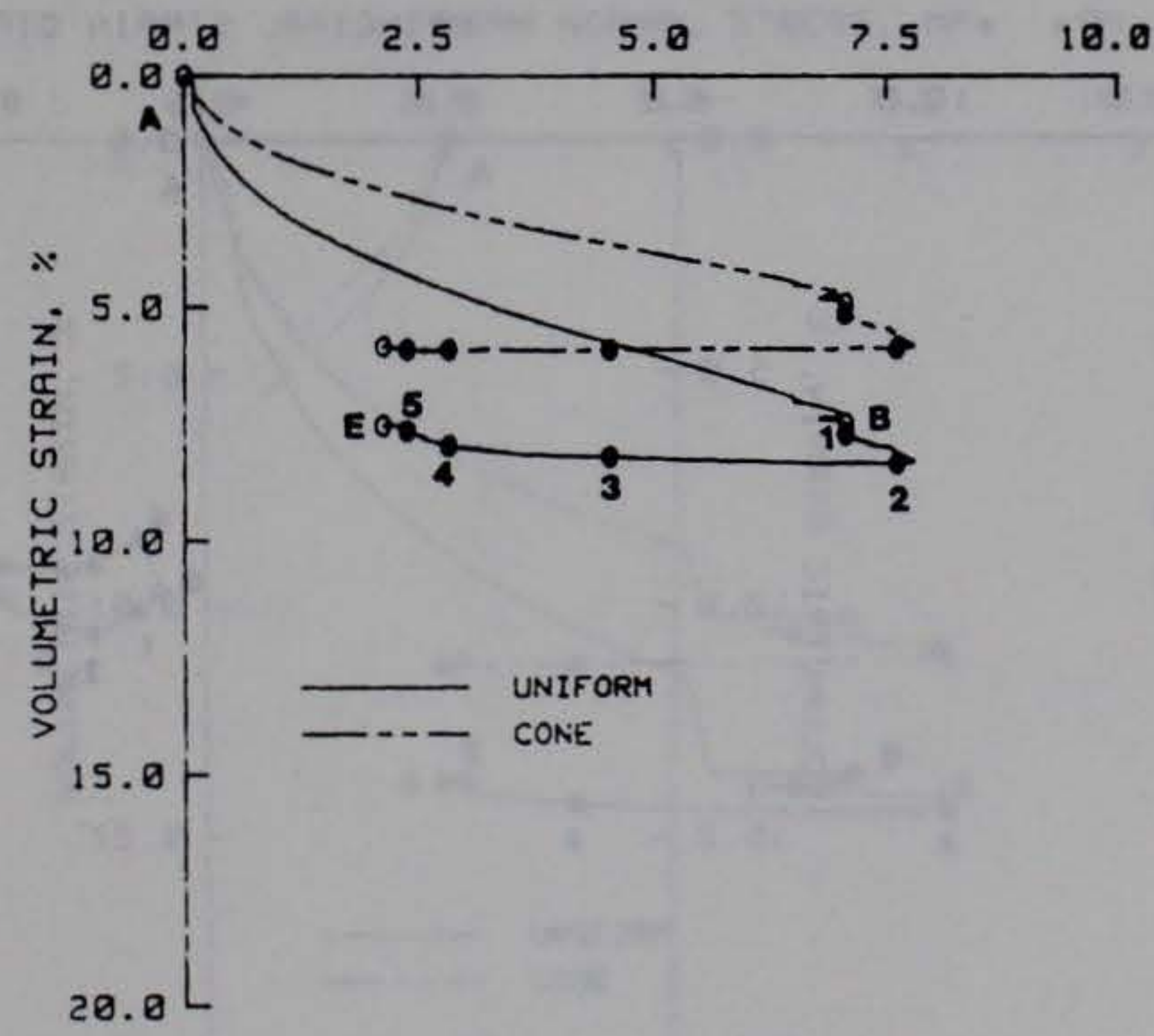

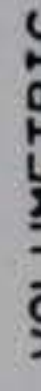

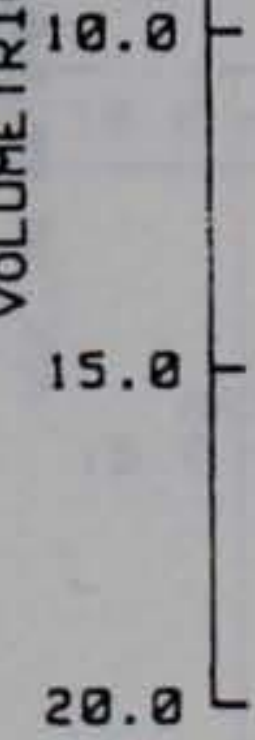




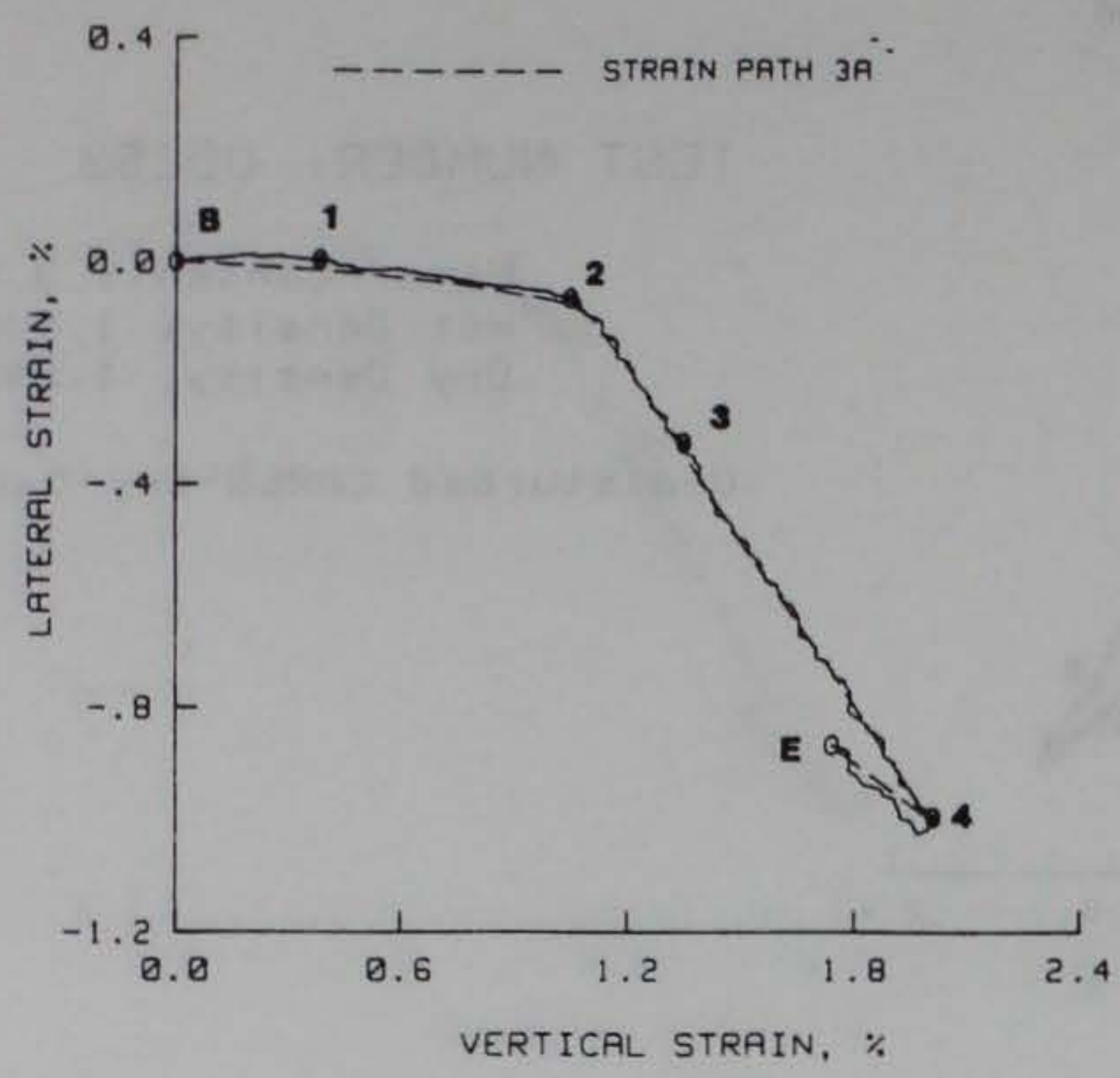

TEST NUMBER: UDCGO

Water Content: $4.32 \%$

Wet Density: $1.963 \mathrm{~g} / \mathrm{cc}$

Dry Density: $1.882 \mathrm{~g} / \mathrm{cc}$

Undisturbed CARES-Dry Sand
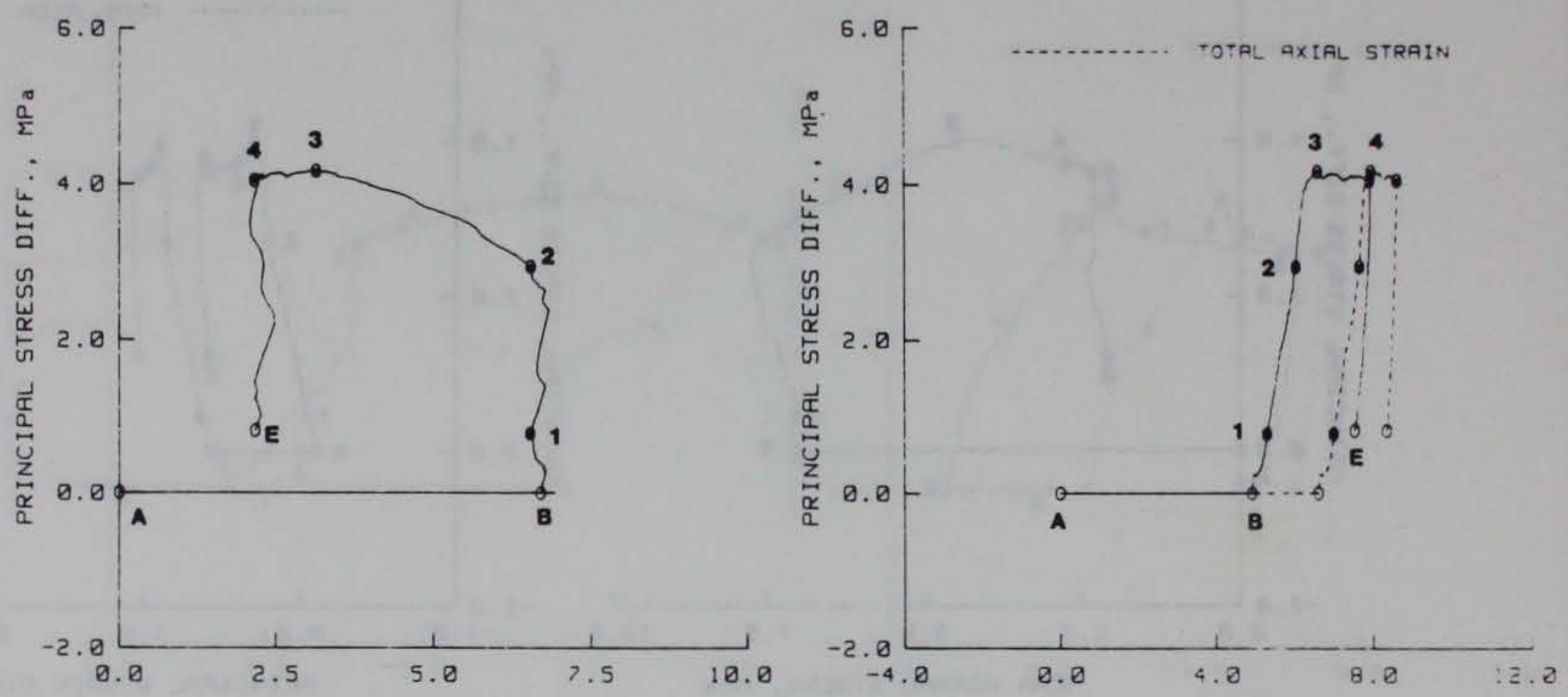

MEAN NORMAL STRESS, MPa
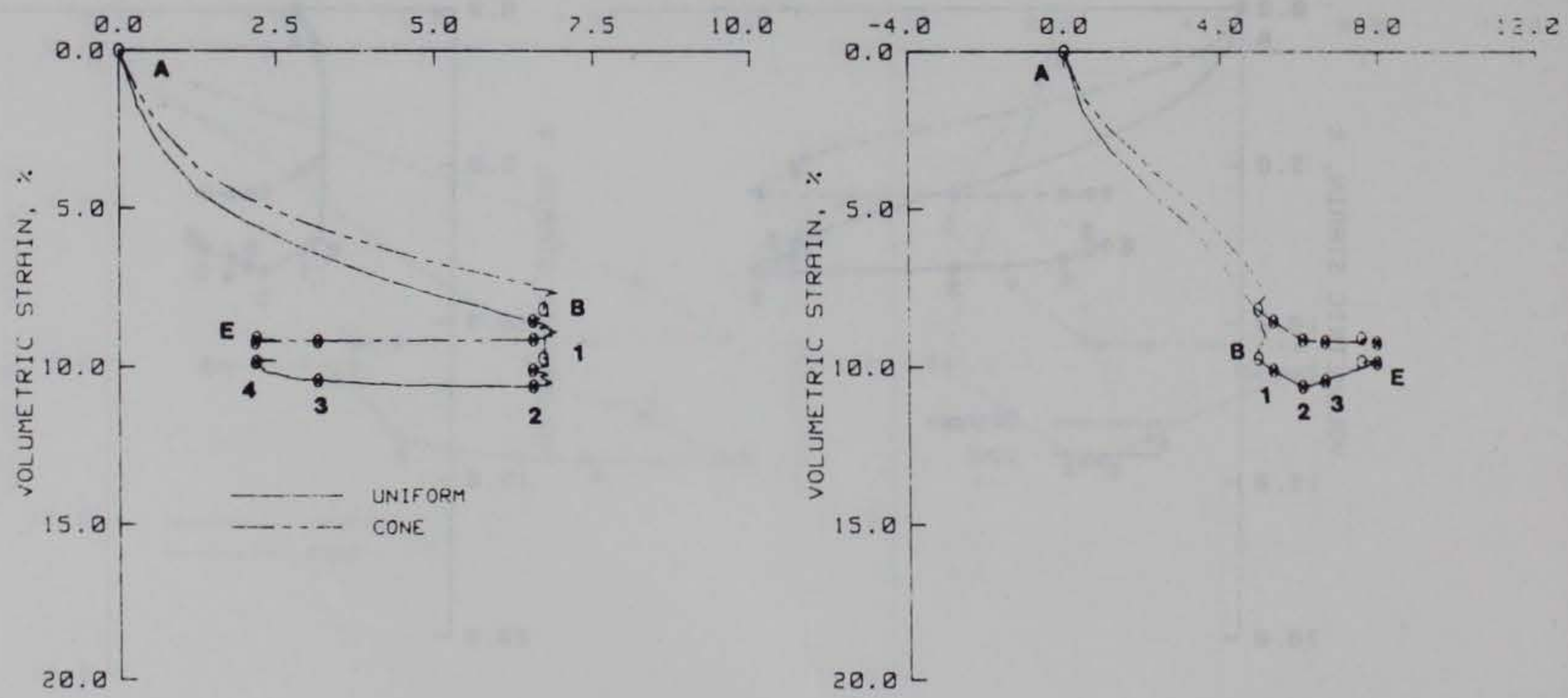


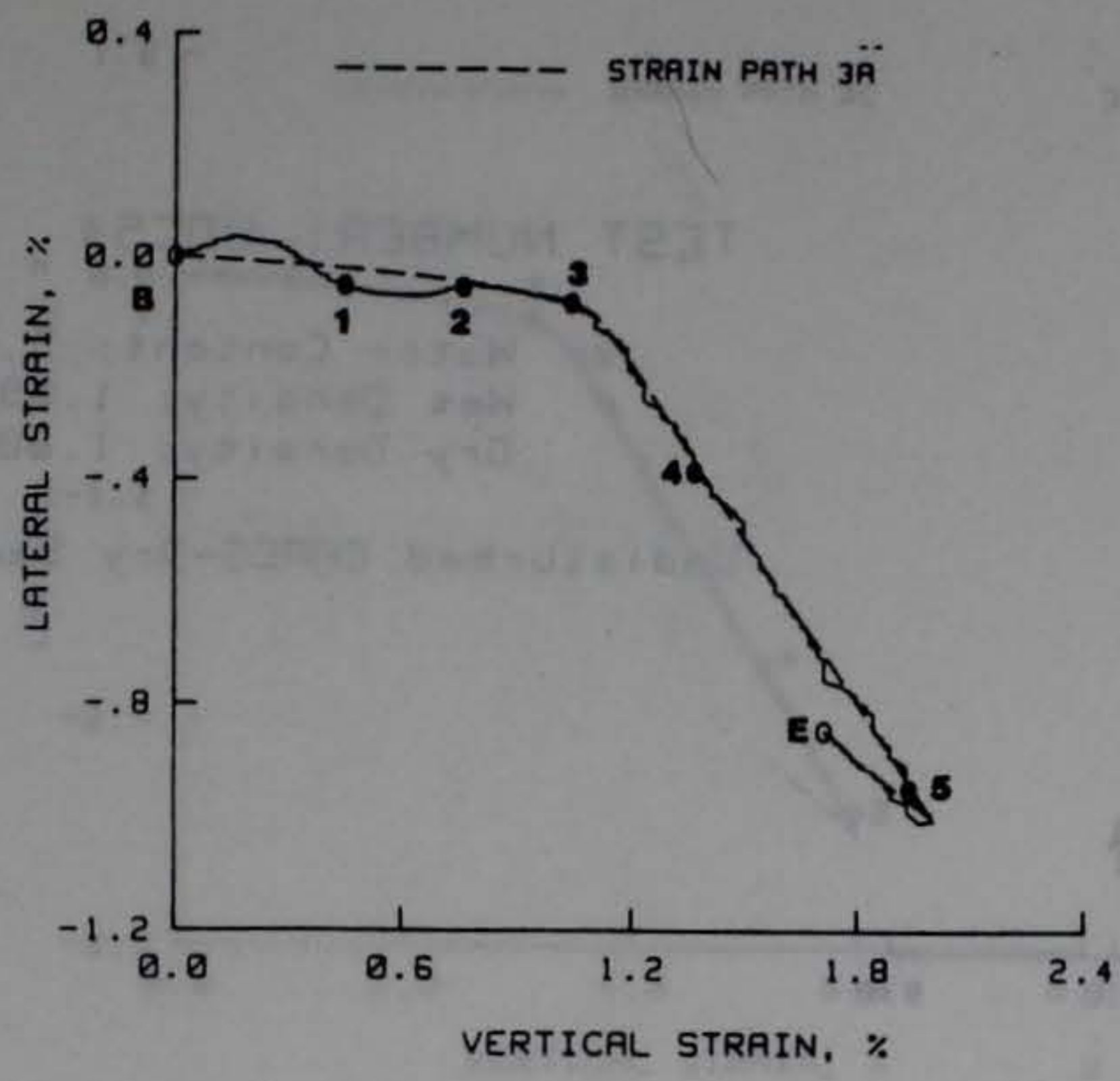

TEST NUMBER: UDC6 1

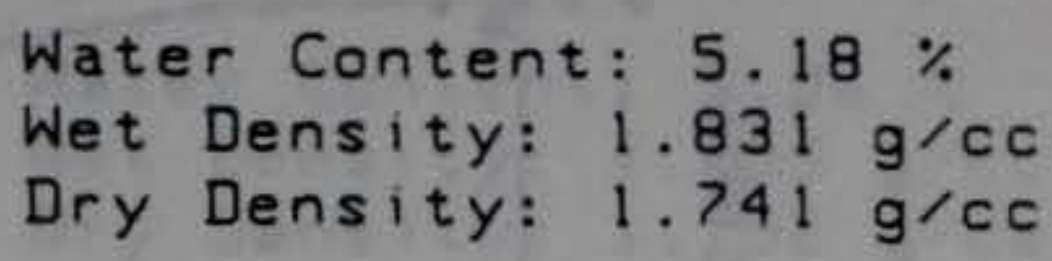

Undisturbed CARES-Dry Sand
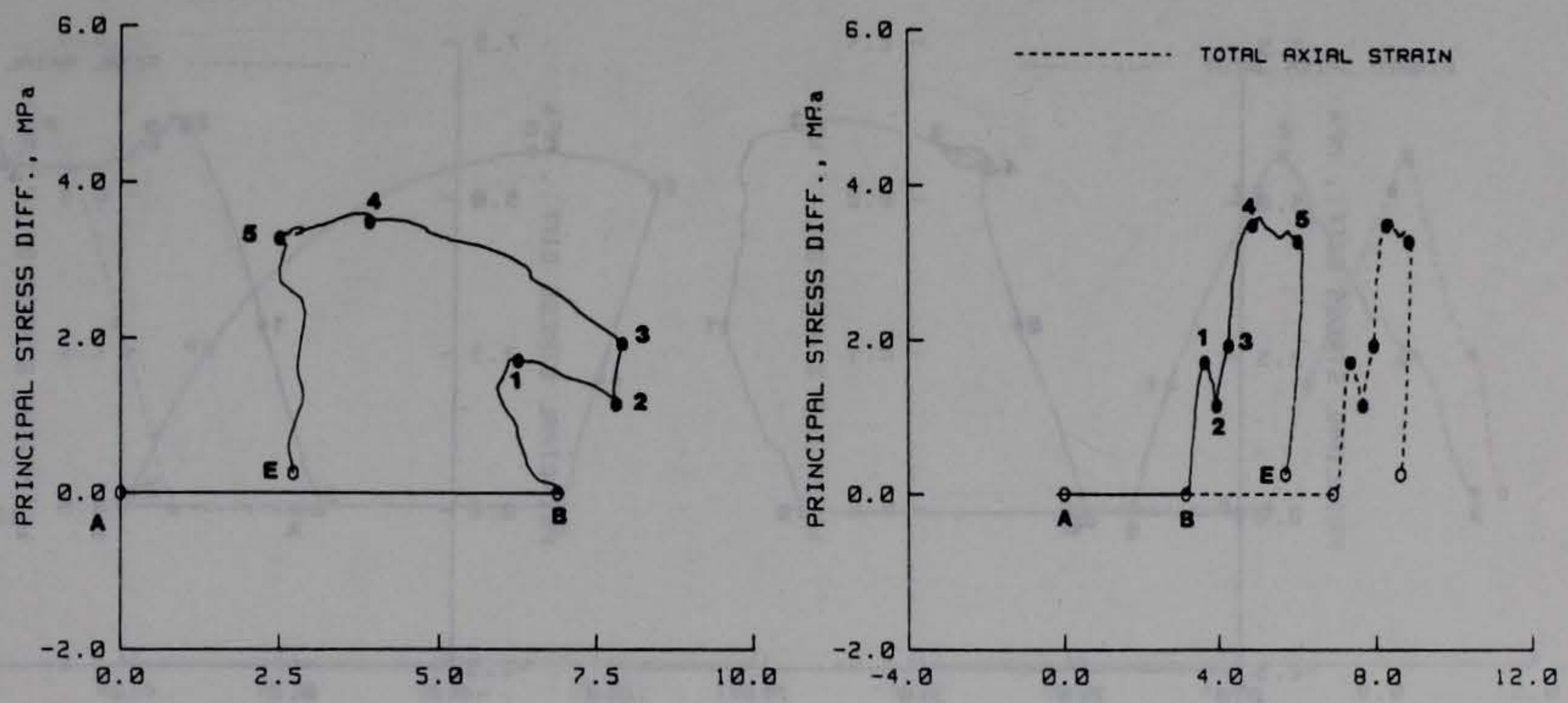

MEAN NORMAL STRESS, MPa

PRINCIPAL STRAIN DIFF., \%
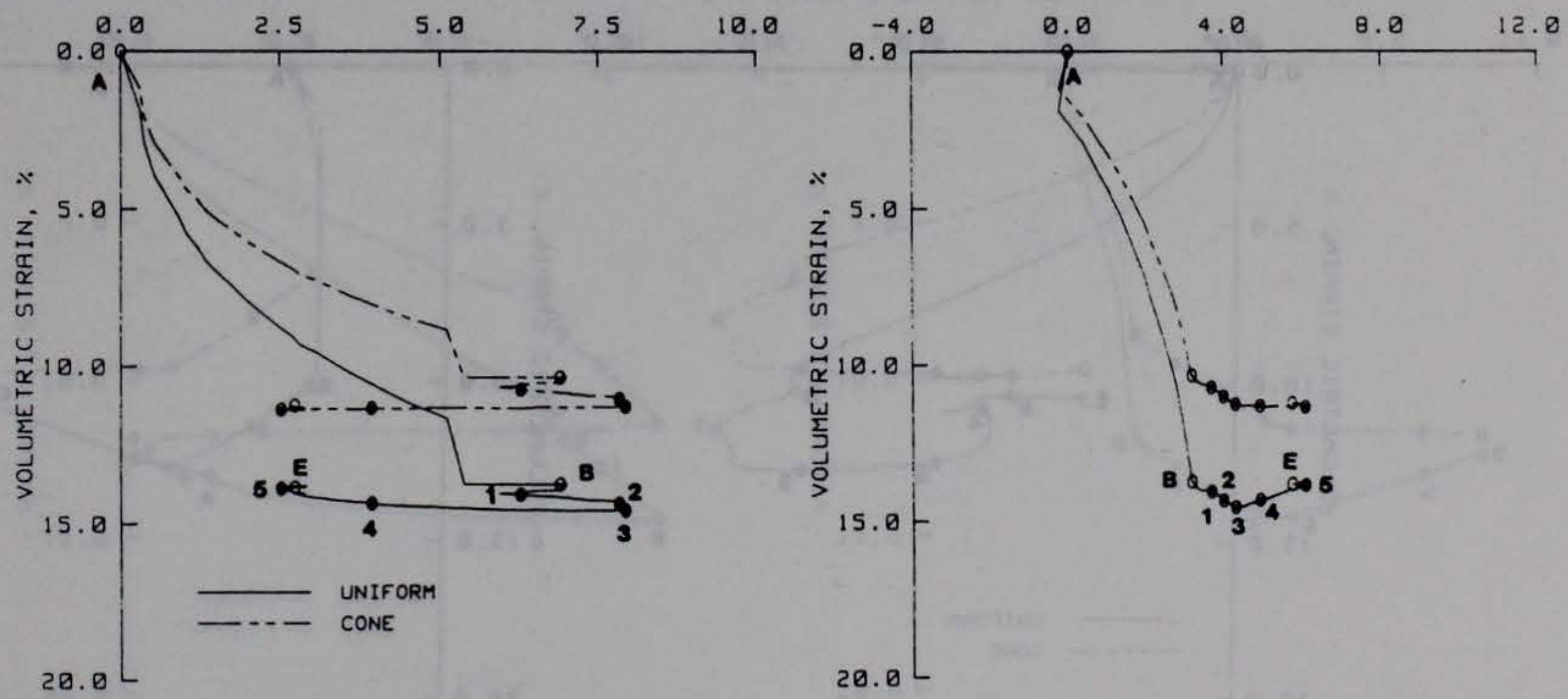


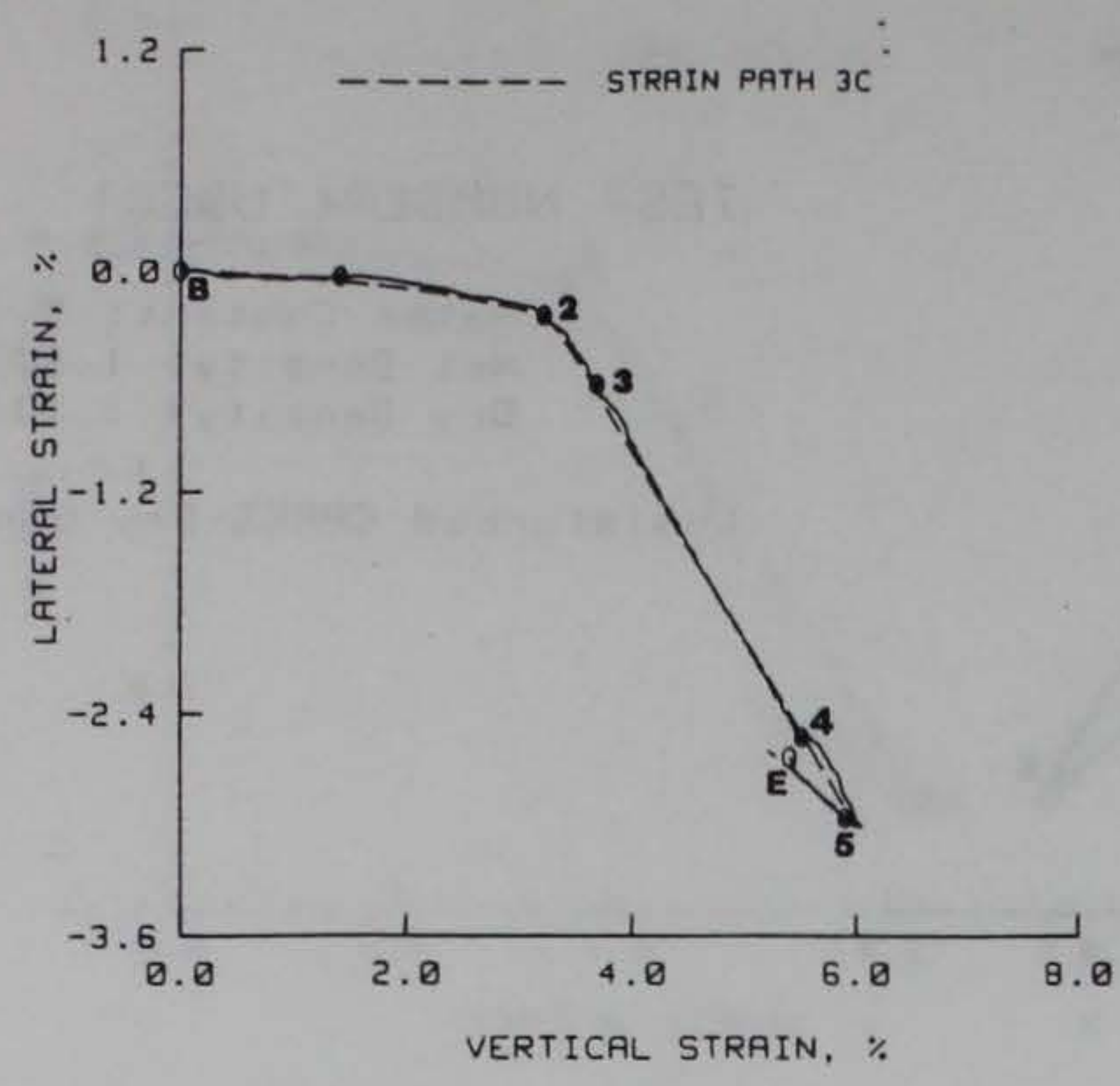

TEST NUMBER: UDC54

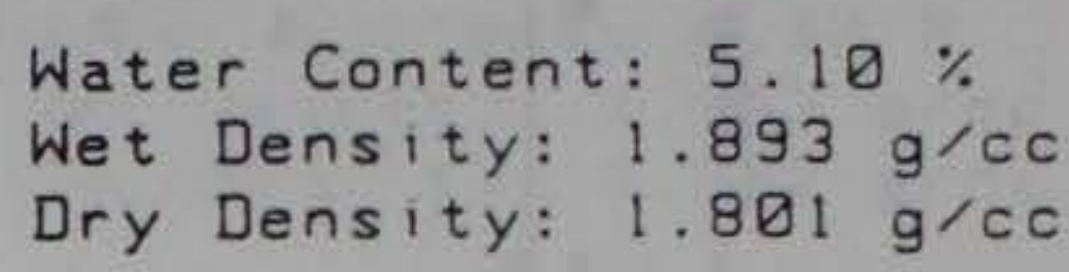

Und isturbed CARES-Dry Sand
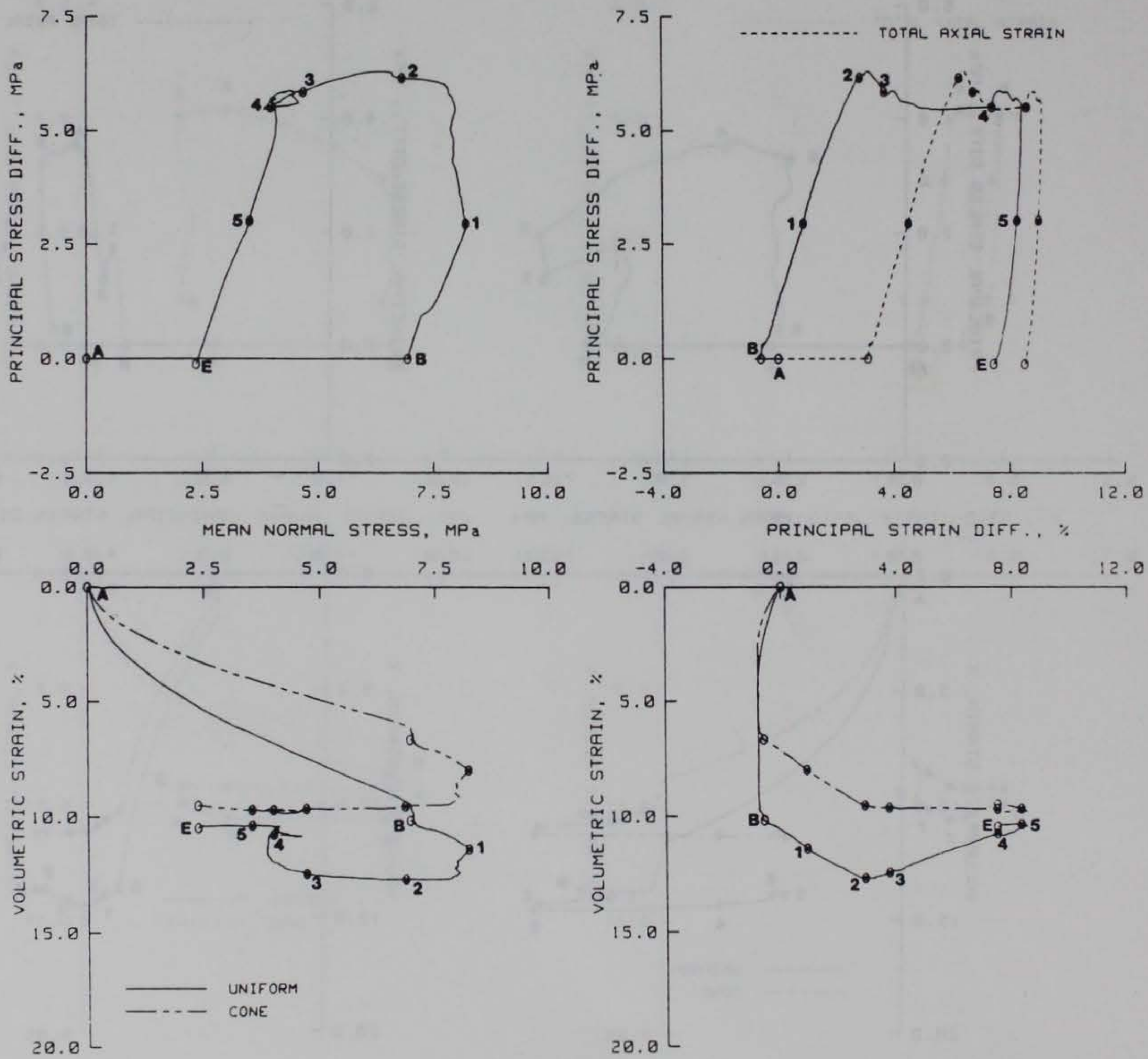


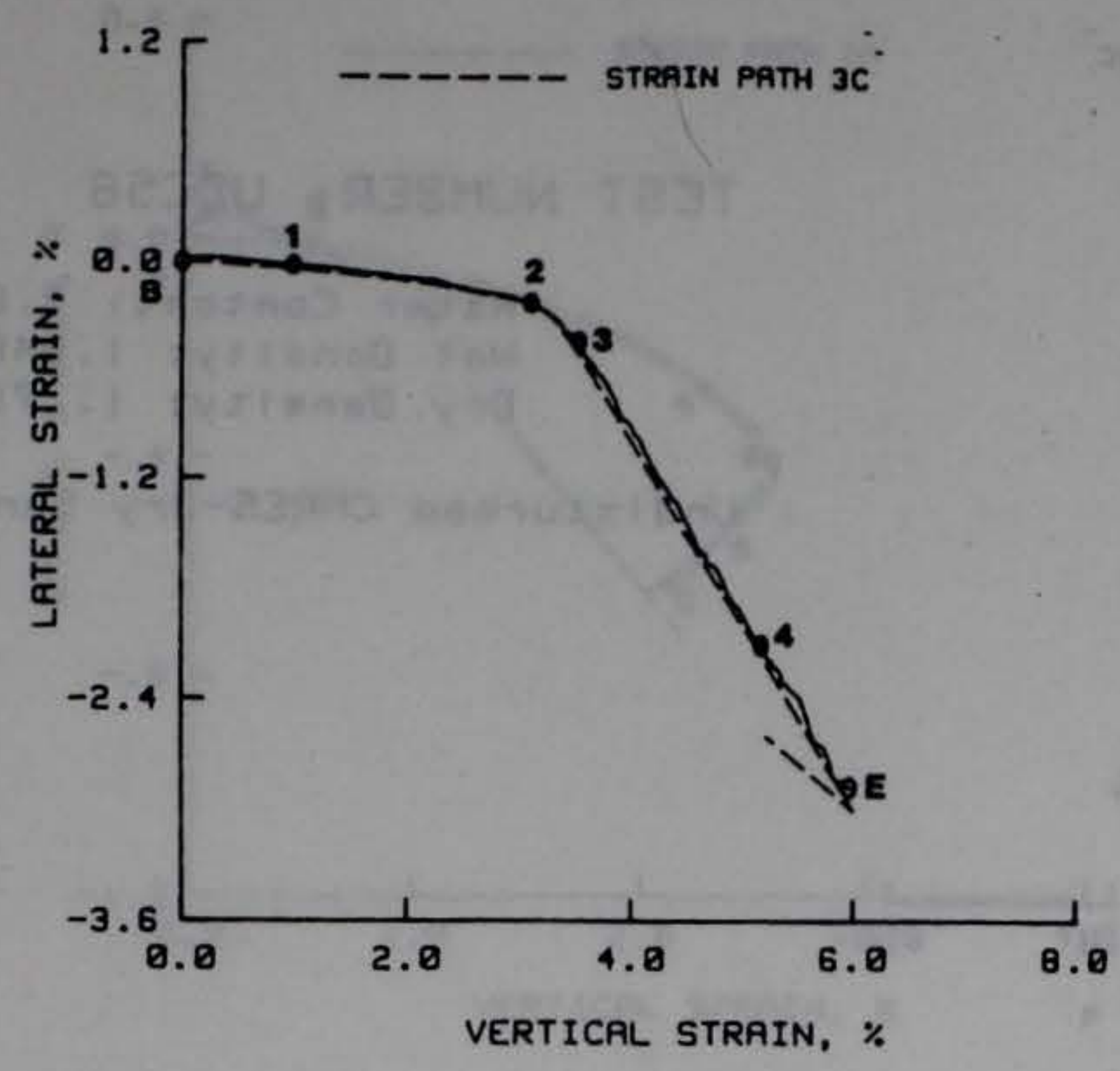

TEST NUMBER: UDC55

Water Content: $5.96 \%$ Wet Density: $1.899 \mathrm{~g} / \mathrm{cc}$ Dry Density: $1.792 \mathrm{~g} / \mathrm{cc}$

Undisturbed CARES-Dry Sand
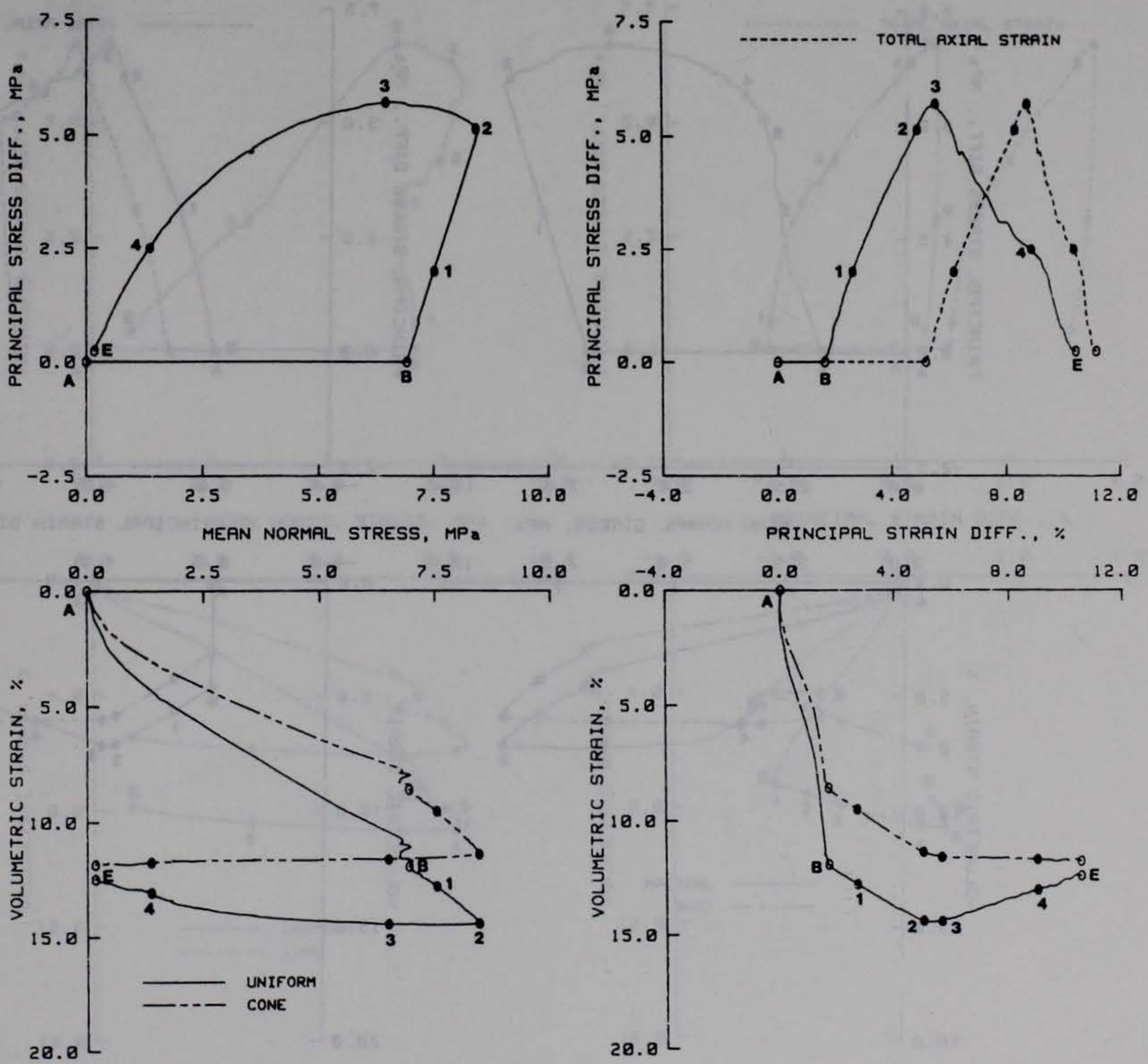


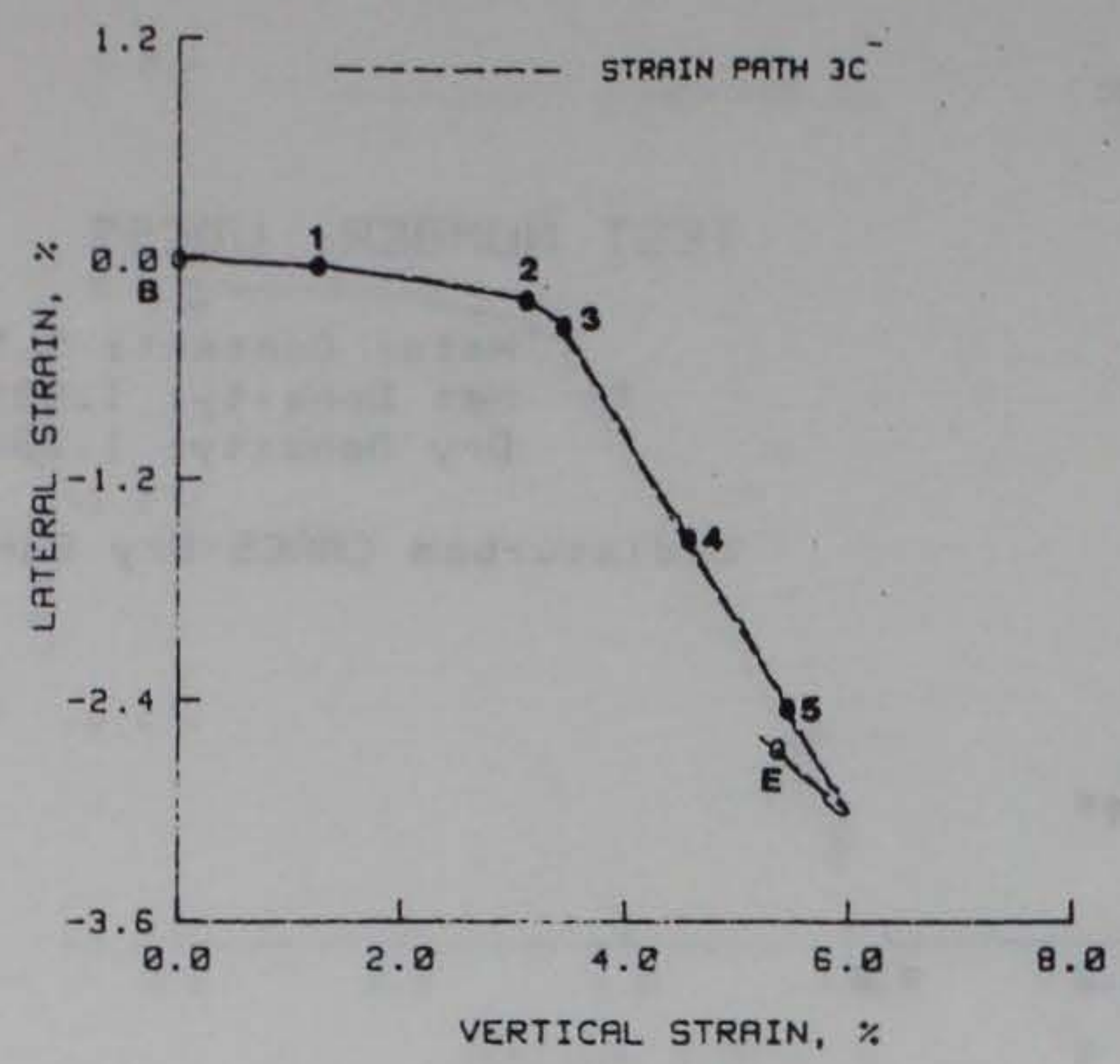

TEST NUMBER: UDC58

Water Content: $3.86 \%$ Wet Density: $1.848 \mathrm{~g} / \mathrm{cc}$

Dry Density: $1.779 \mathrm{~g} / \mathrm{cc}$

Und isturbed CARES-Dry Sand
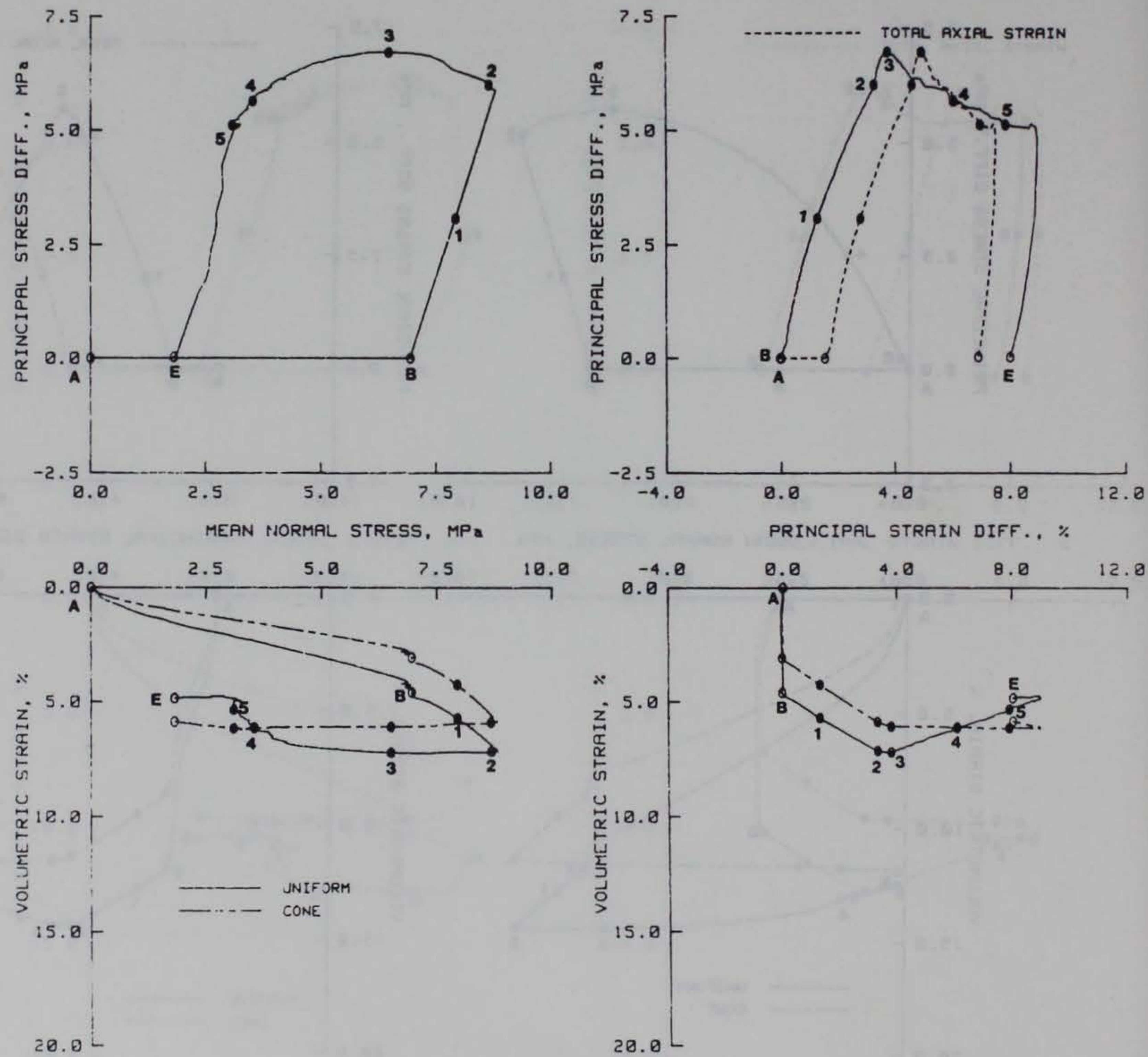


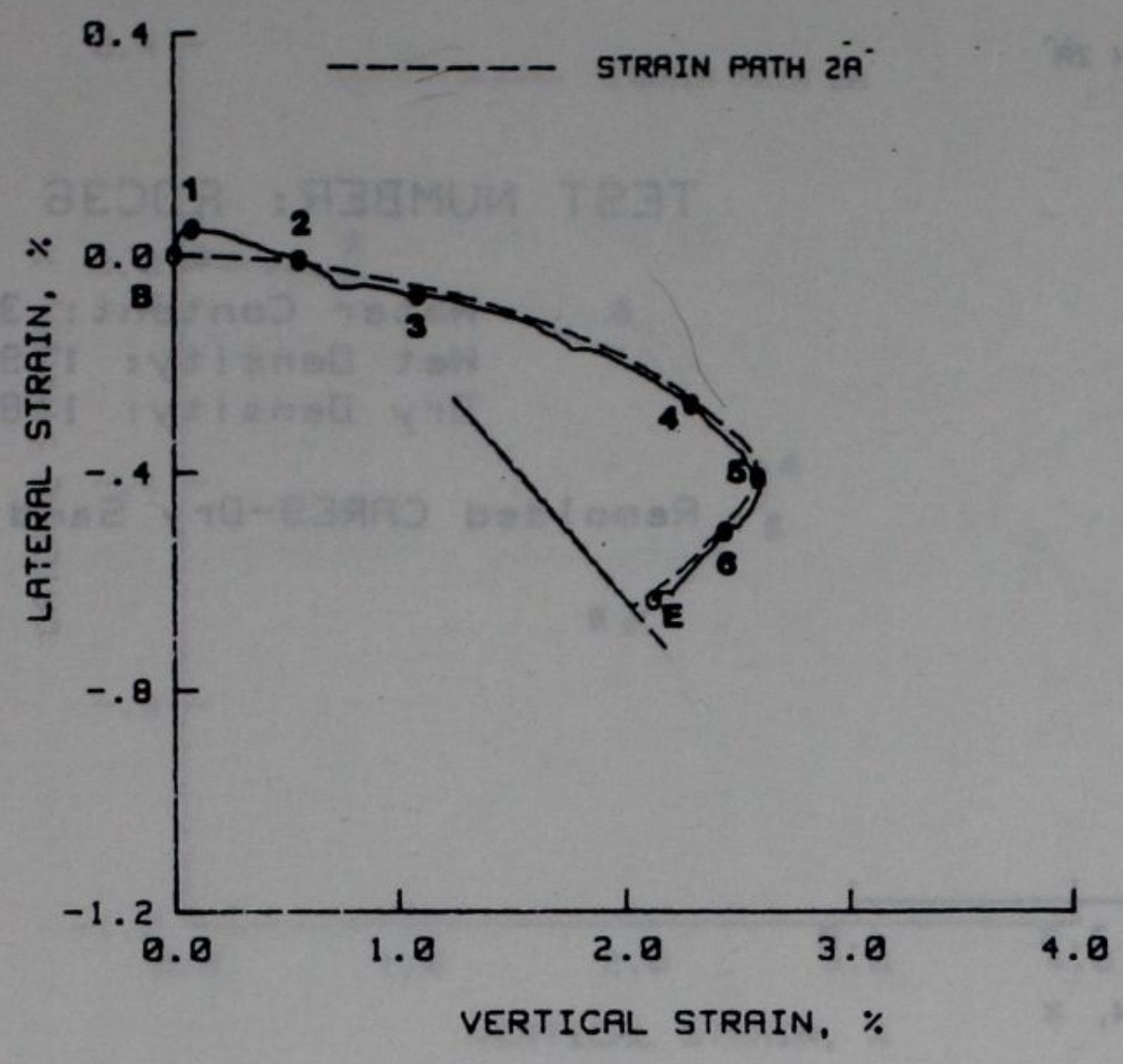

TEST NUMBER: RDC35

Water Content: $4.01 \%$ Wet Density: $1.904 \mathrm{~g} / \mathrm{Cc}$ Dry Density: $1.831 \mathrm{~g} / \mathrm{cc}$

Remolded CARES-Dry Sand
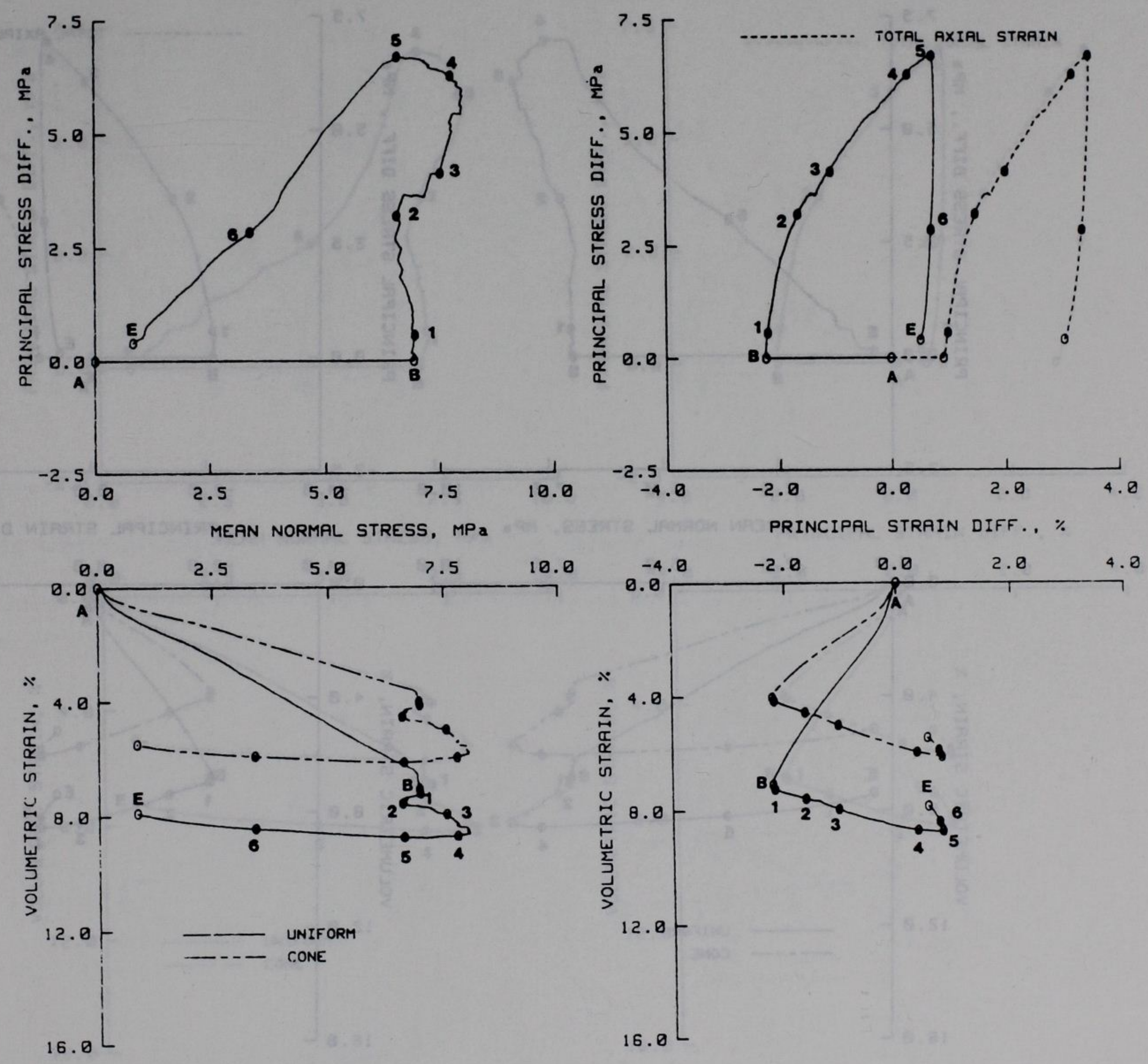


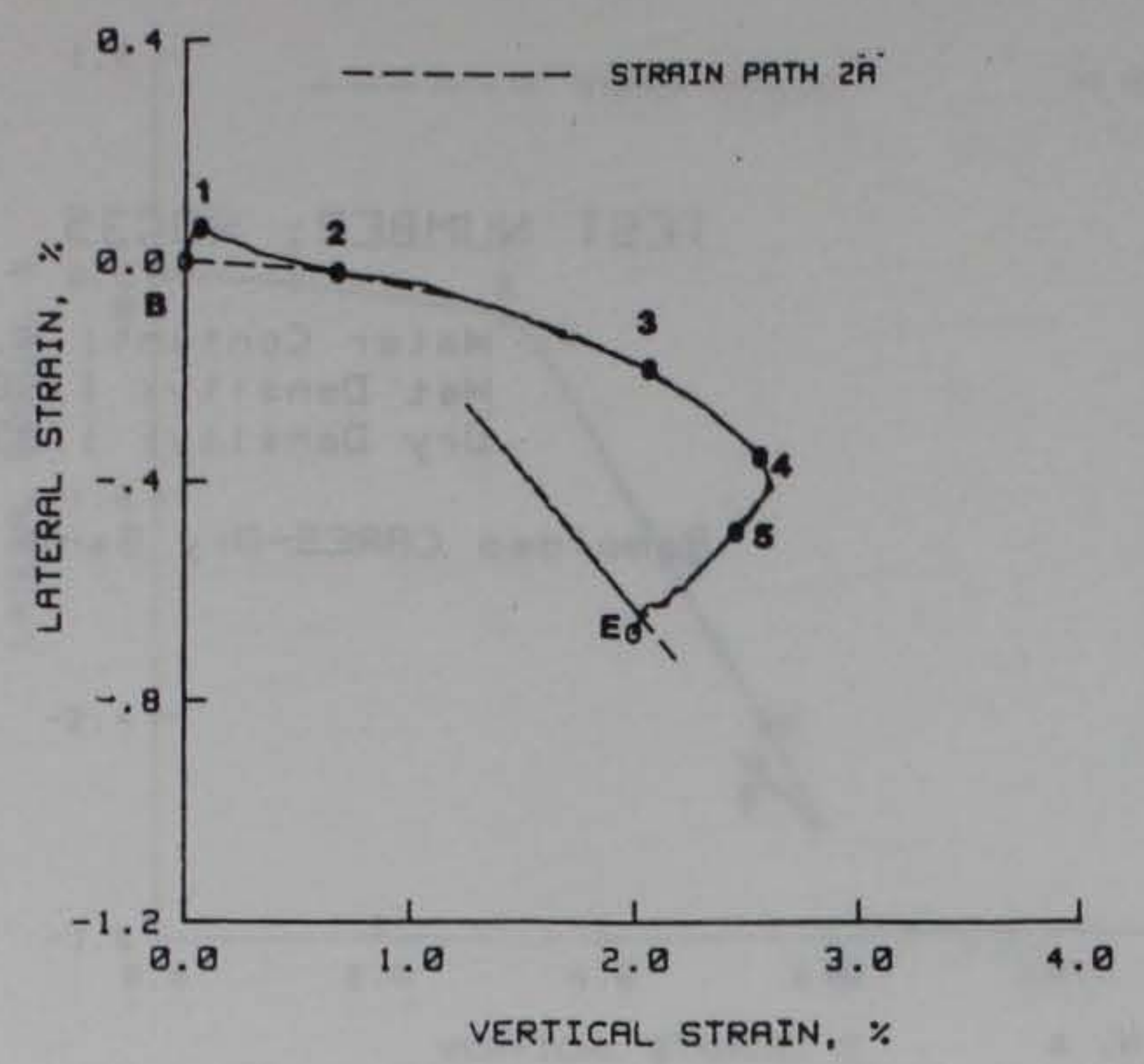

TEST NUMBER: RDC36

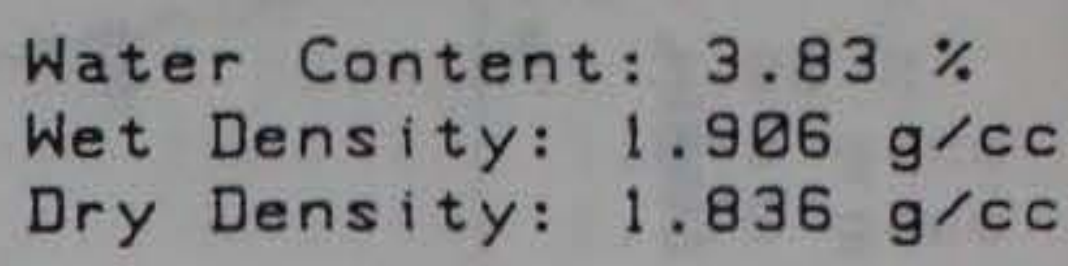

Remolded CARES-Dry Sand
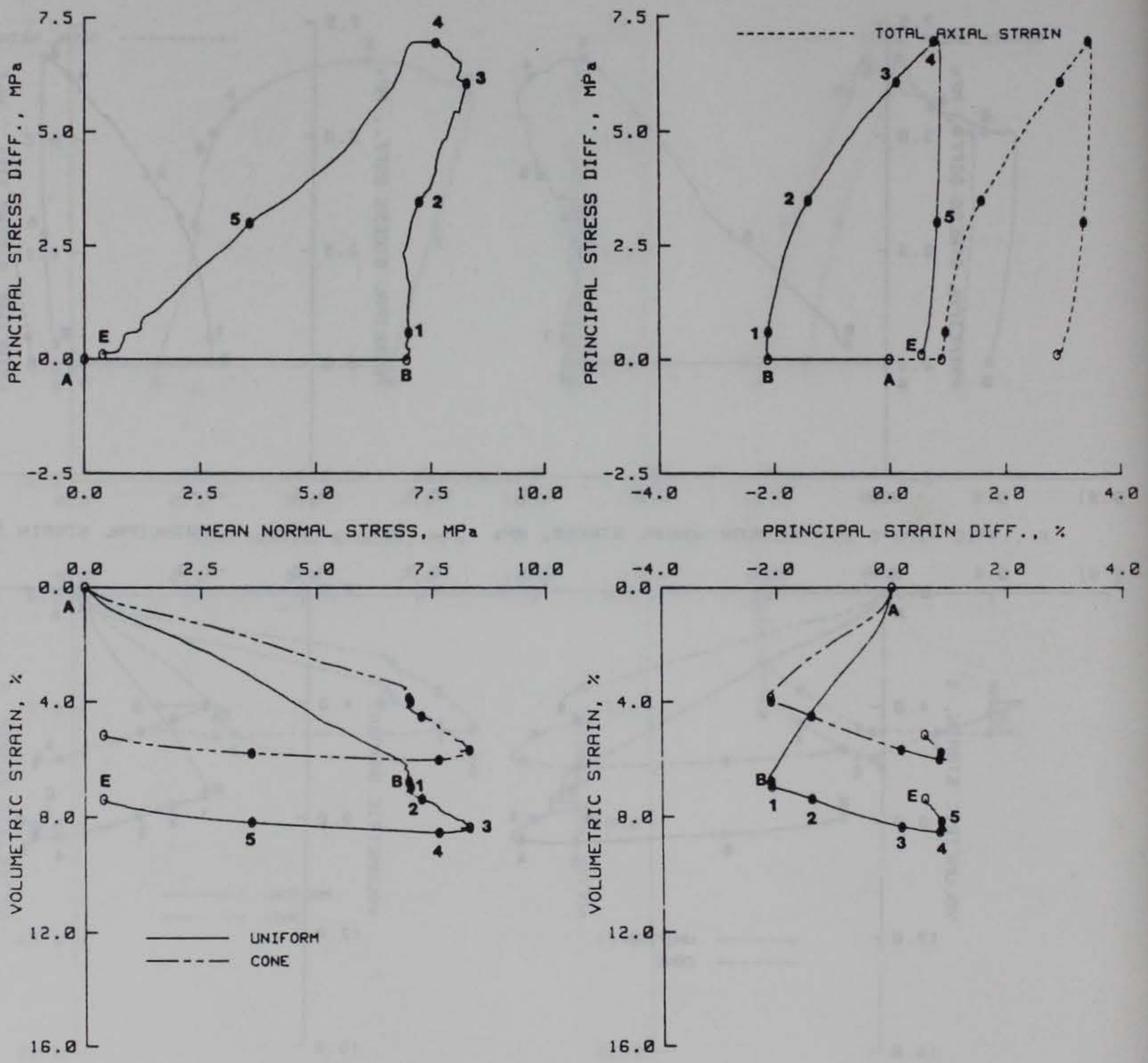


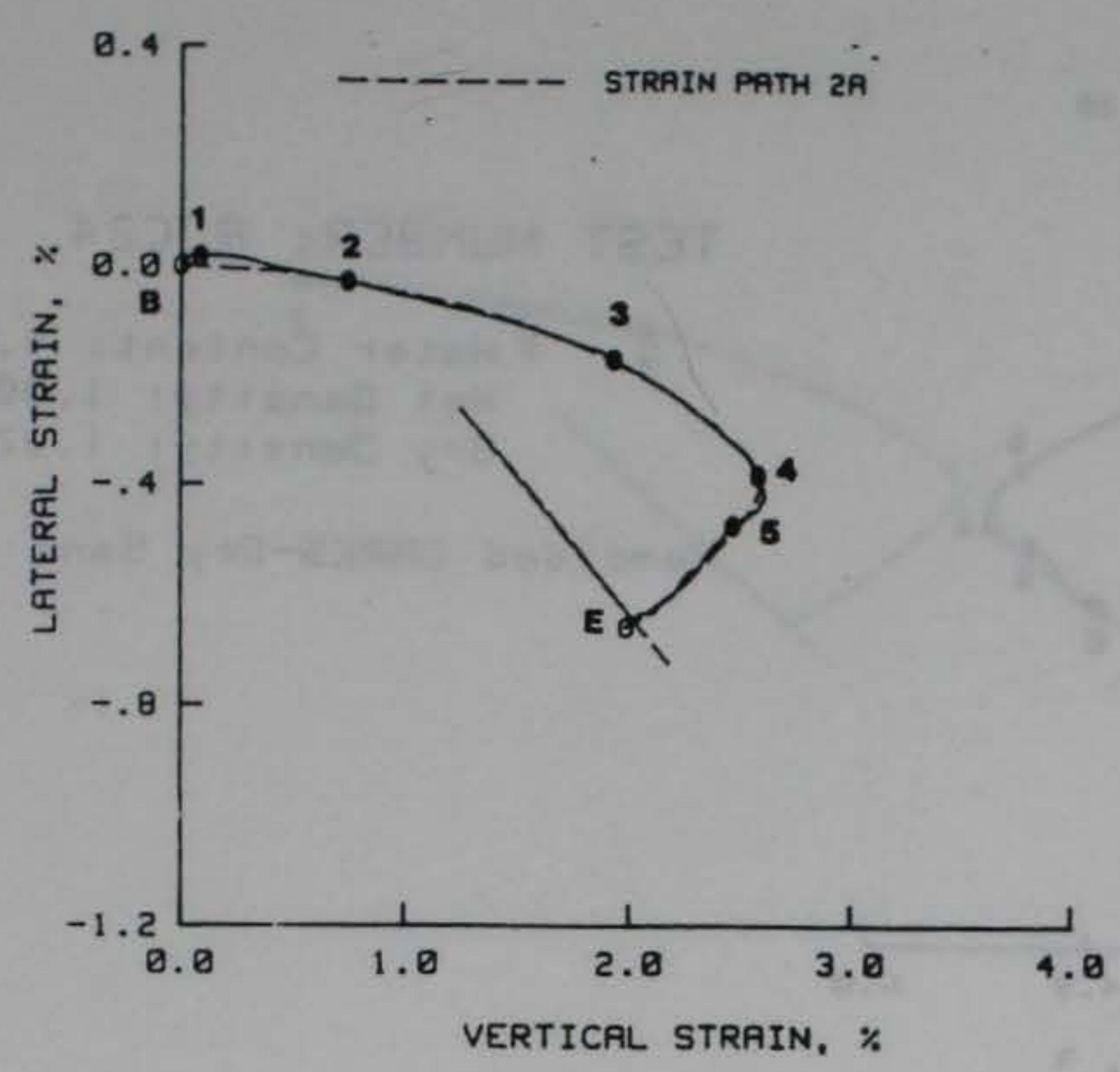

TEST NUMBER: RDC37

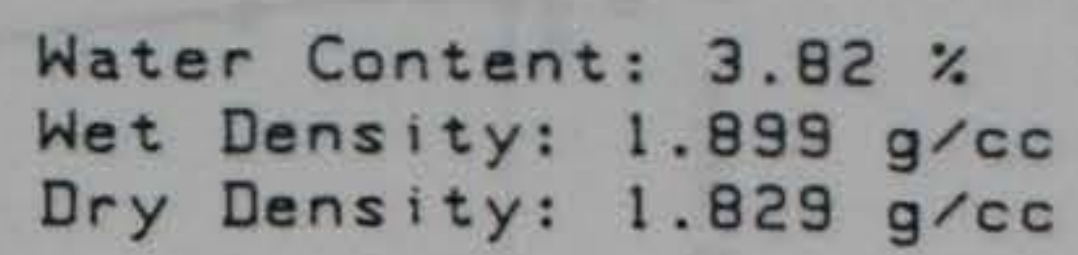

Remolded CARES-Dry Sand

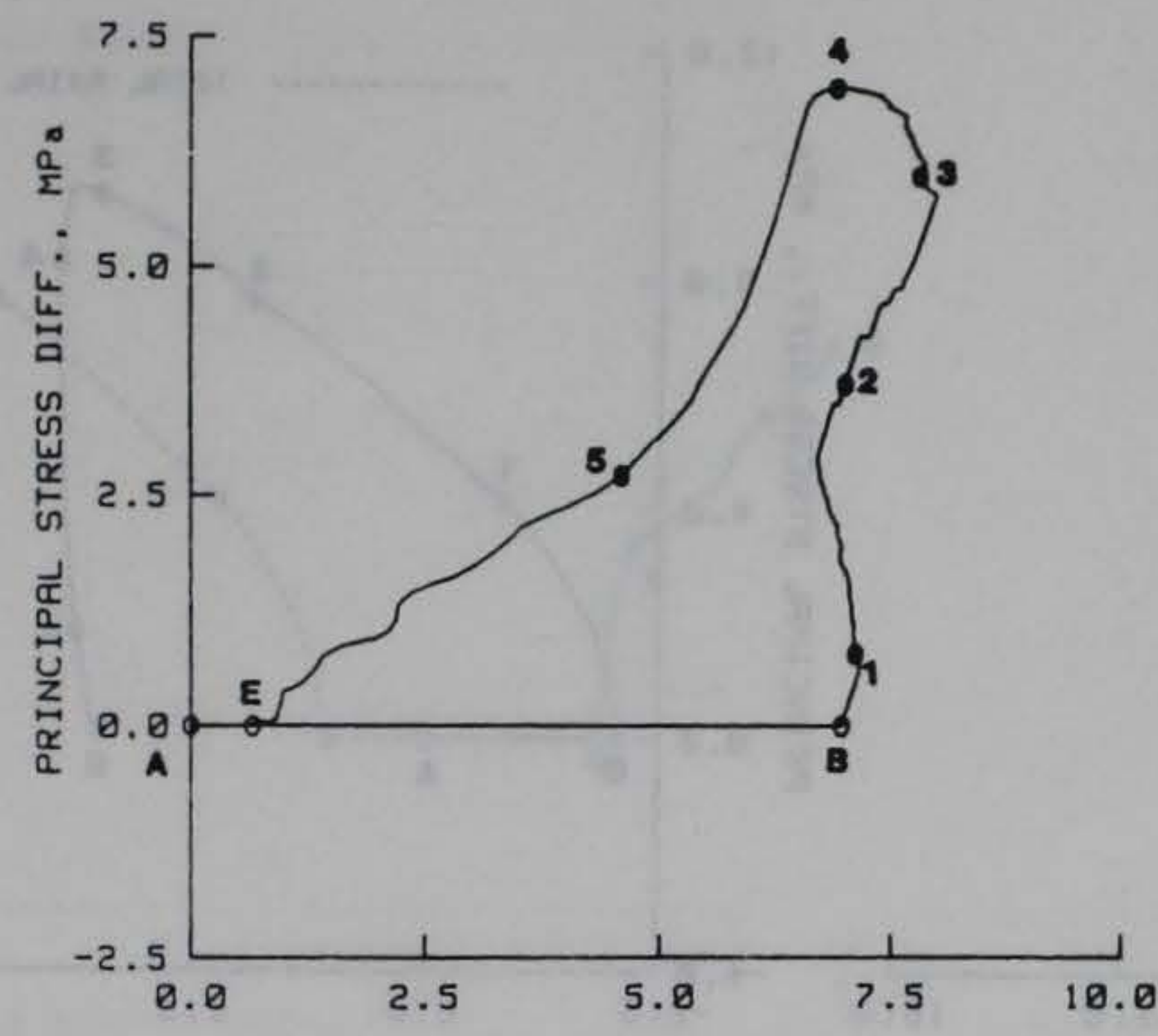

MEAN NORMAL STRESS, MPa
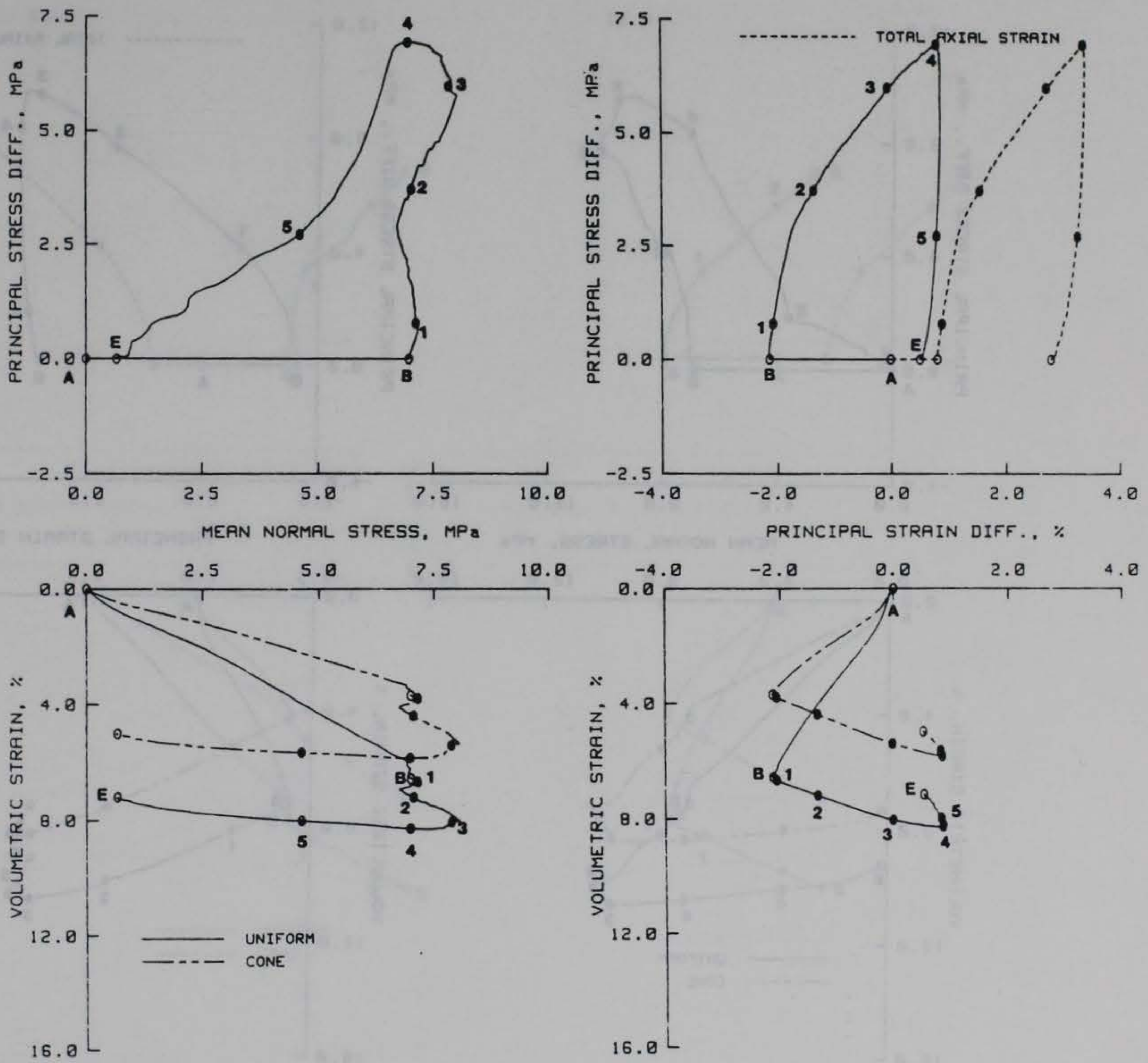


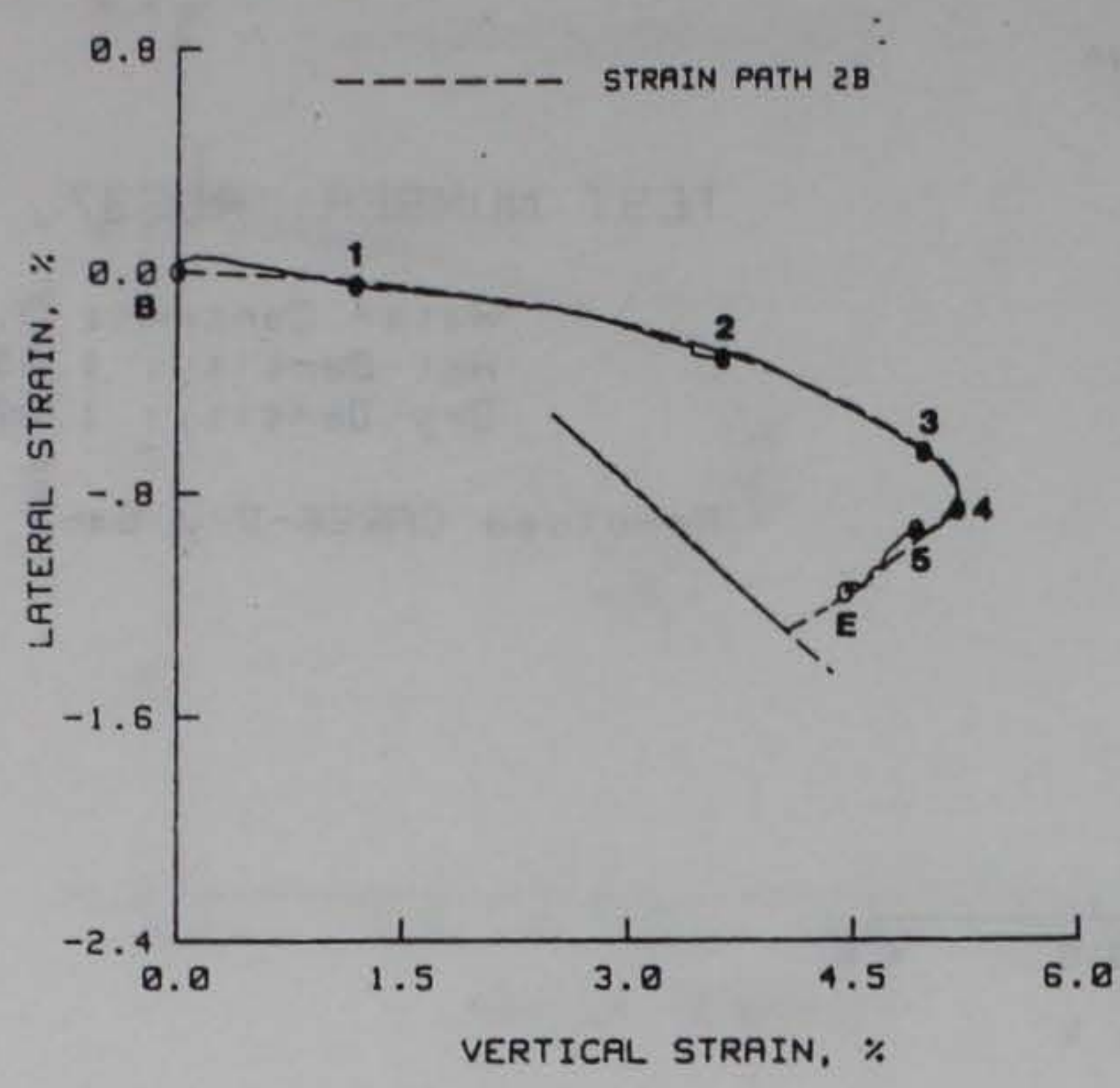

TEST NUMBER: RDC24

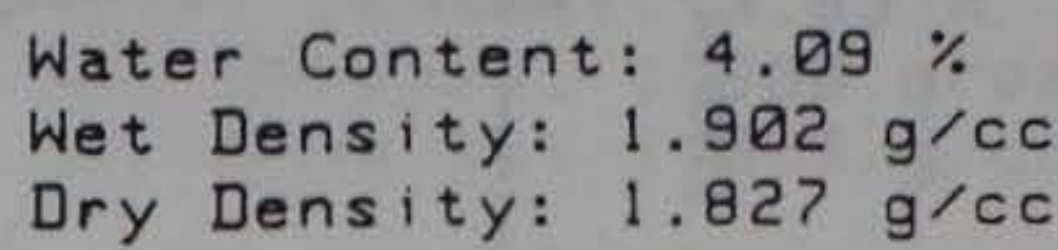

Remolded CARES-Dry Sand
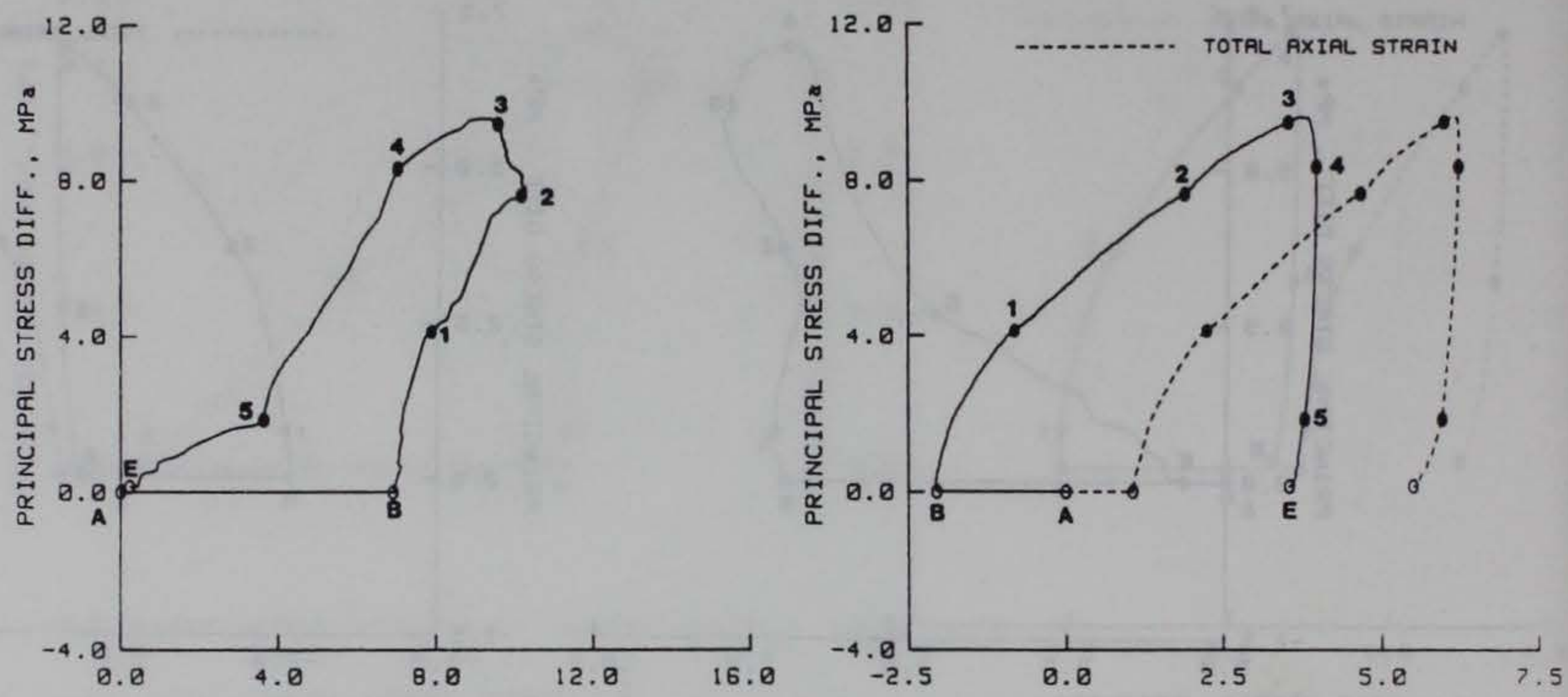

MEAN NORMAL STRESS, MPa
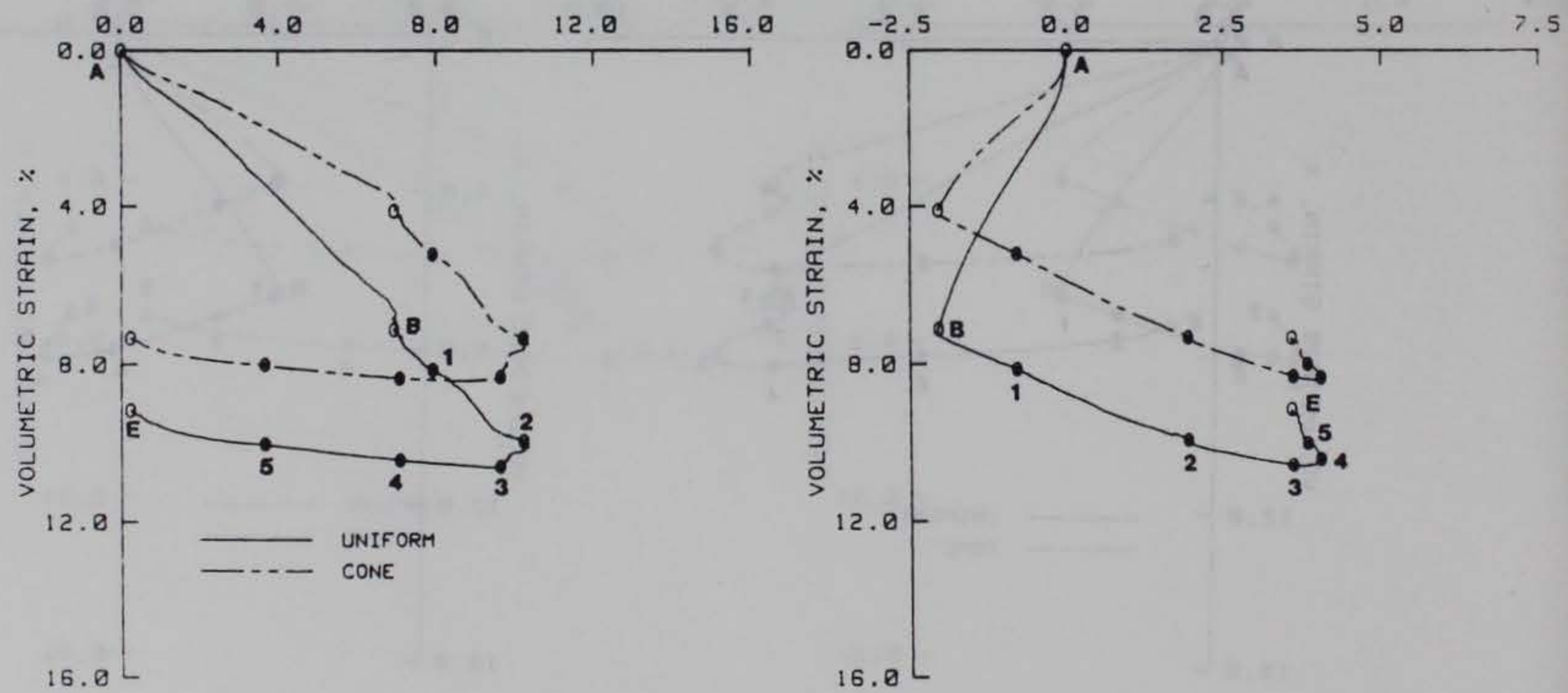


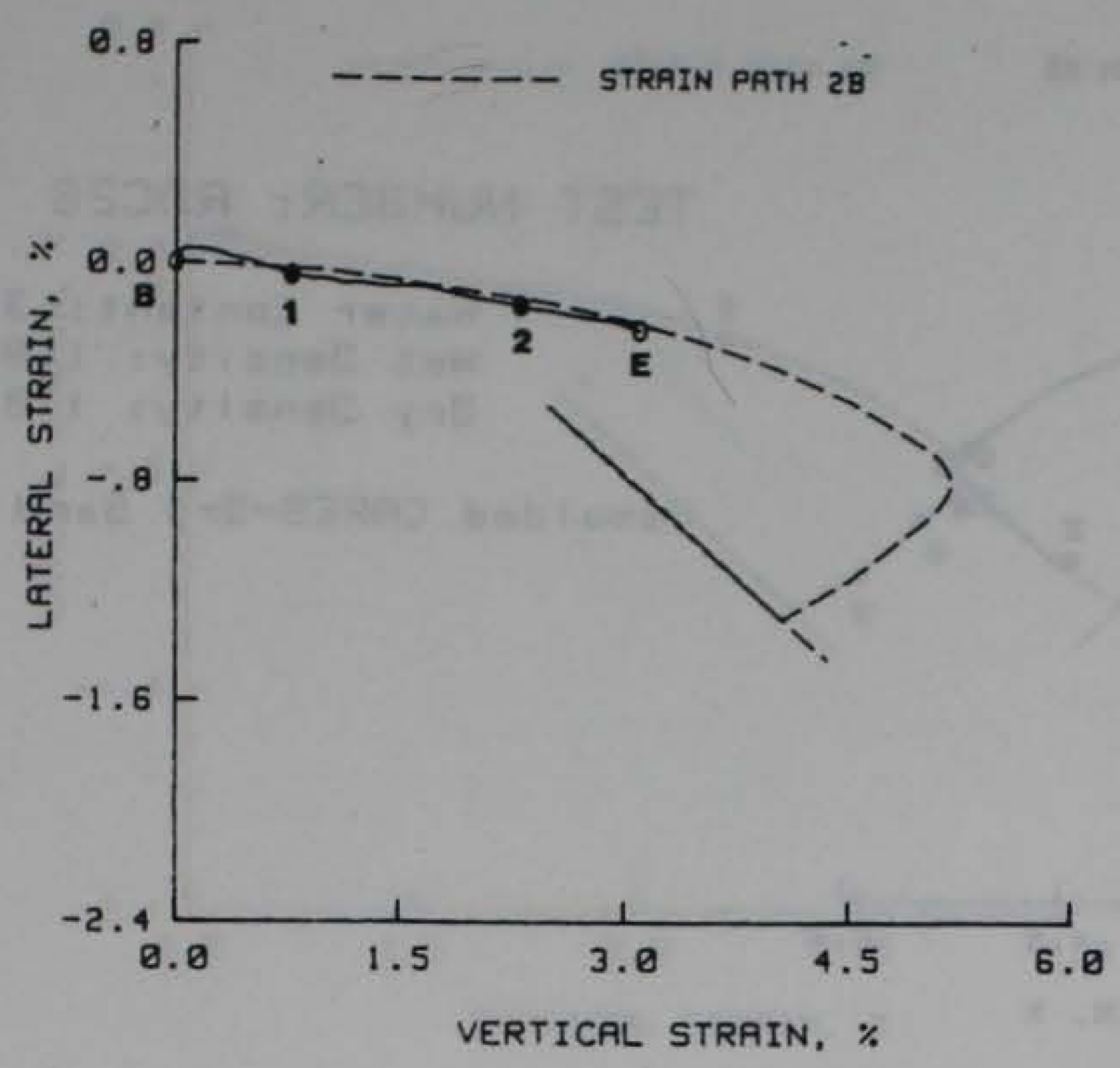

TEST NUMBER: RDC25

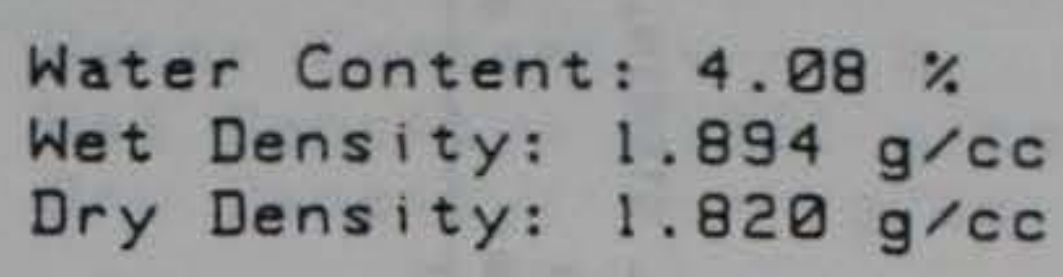

Remolded CARES-Dry Sand
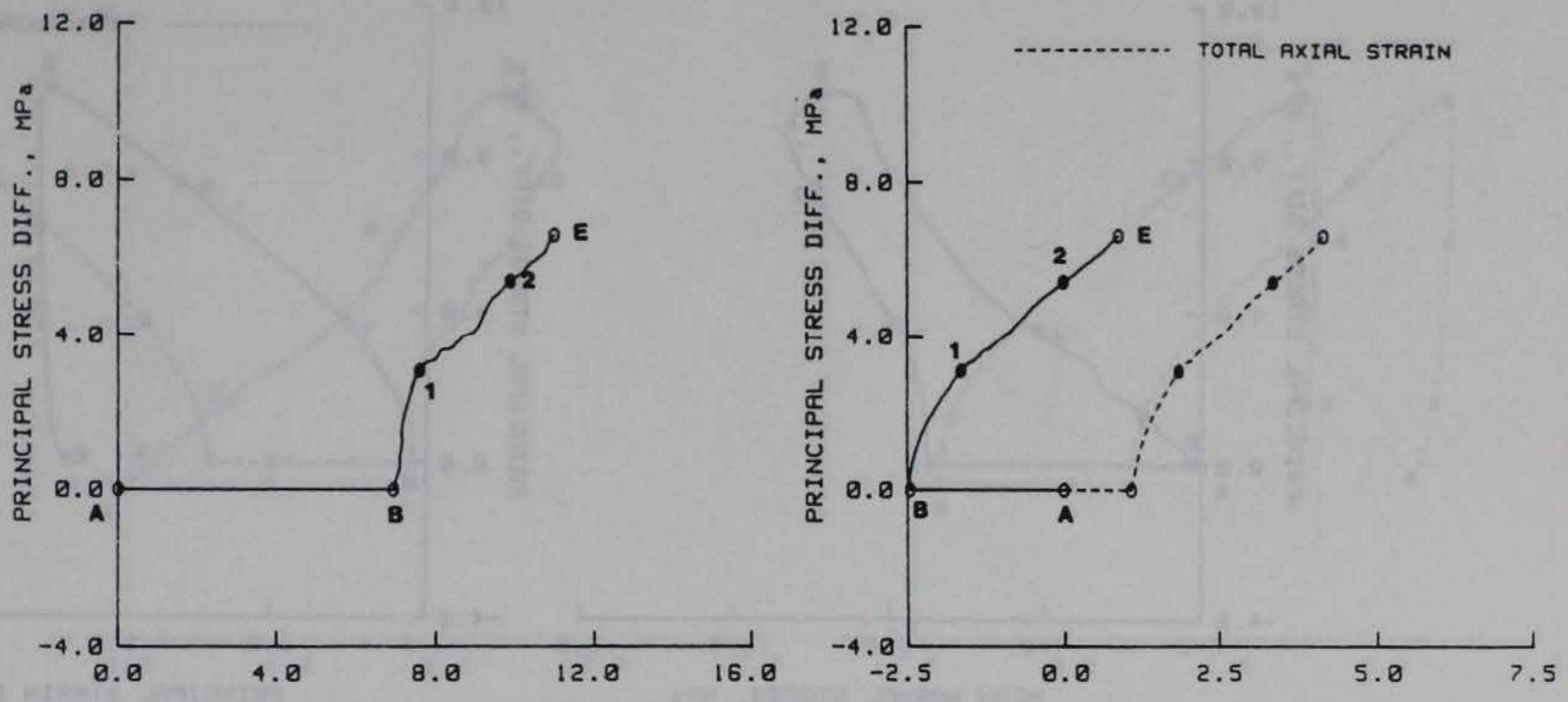

MEAN NORMAL STRESS, MPa
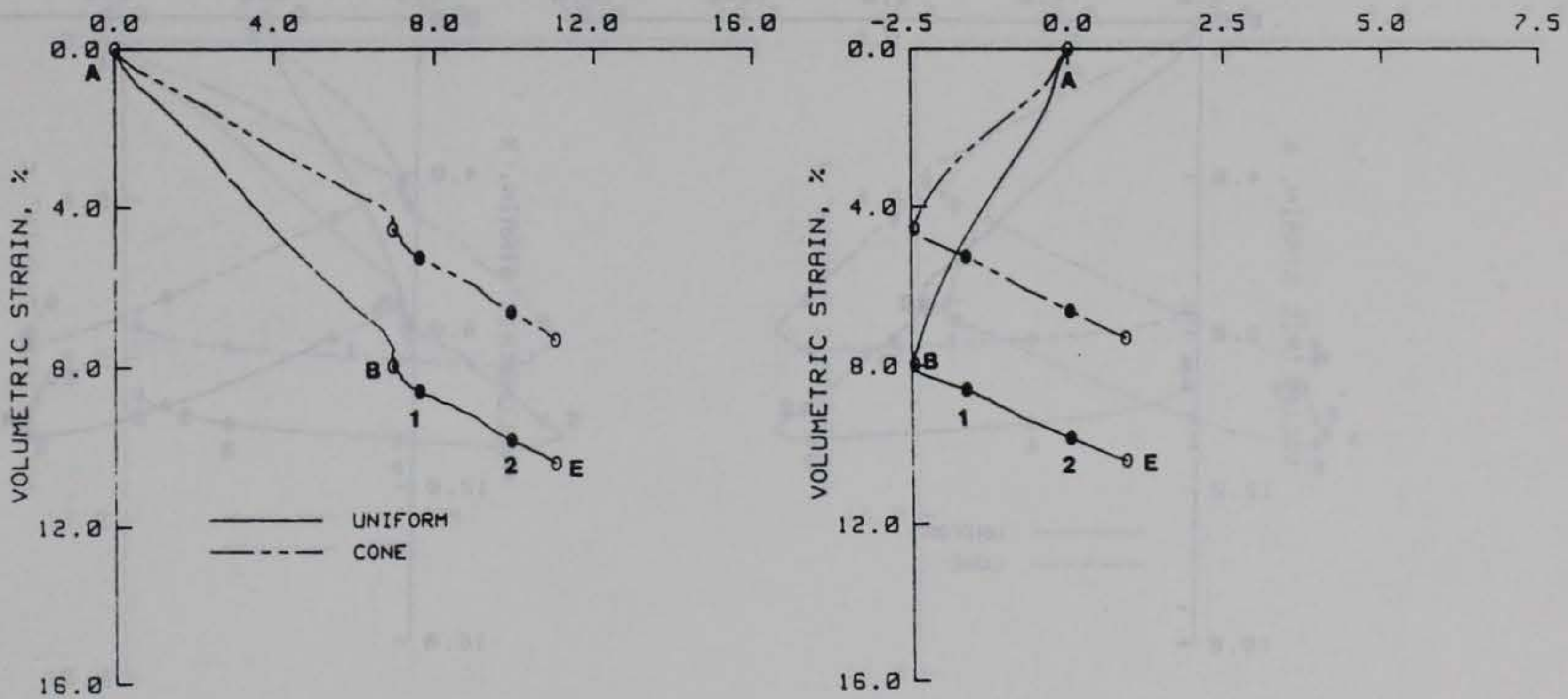


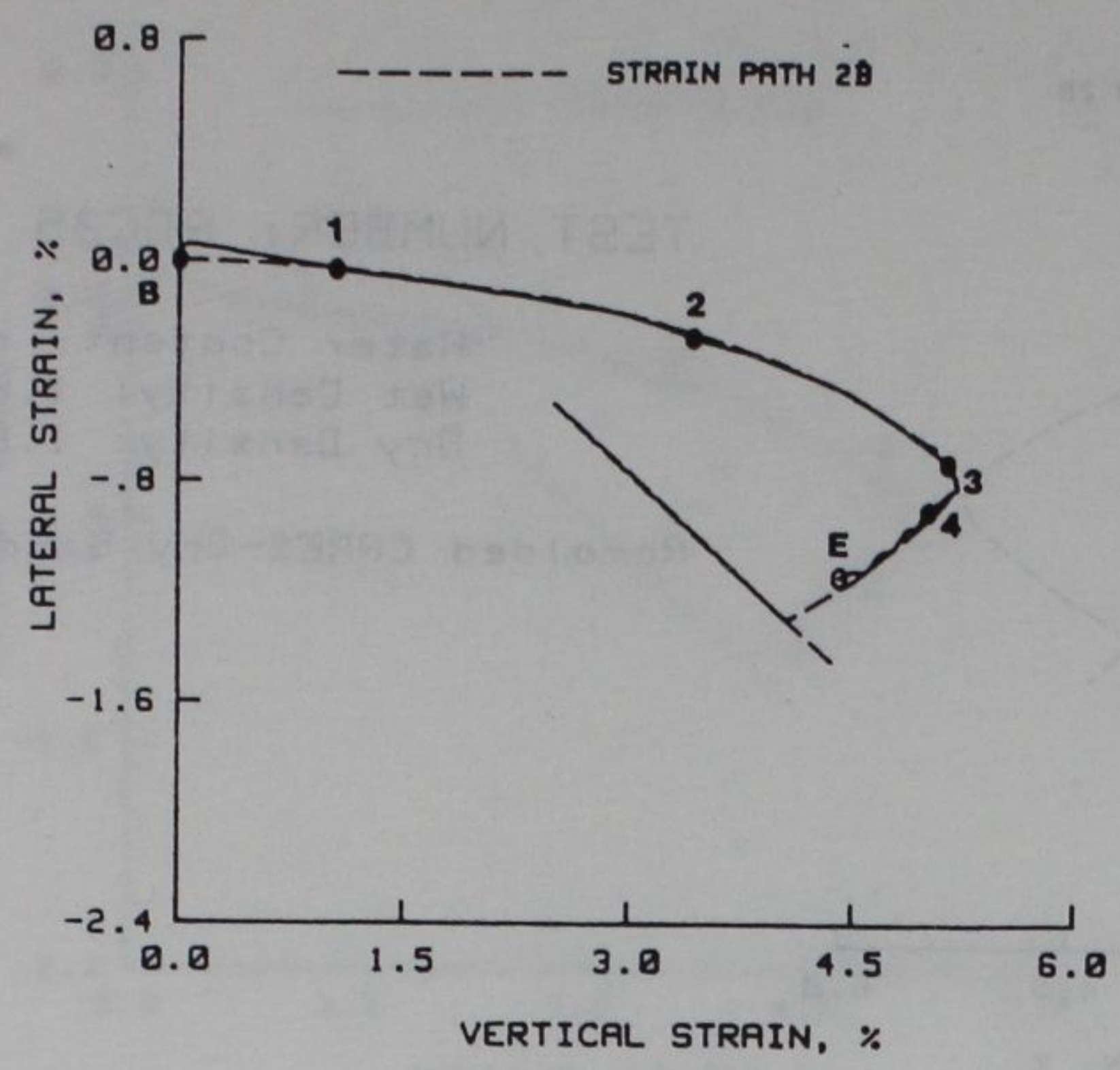

TEST NUMBER: RDC26

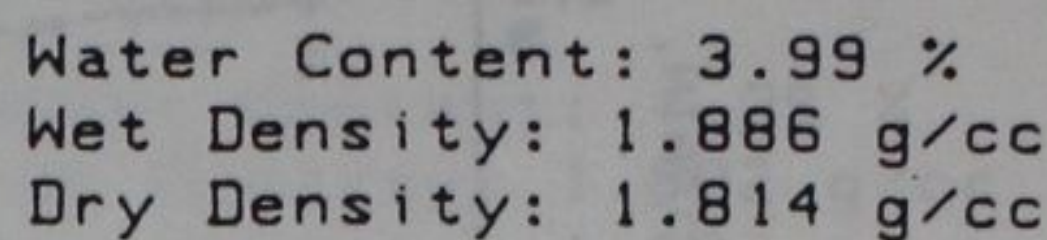

Remolded CARES-Dry Sand
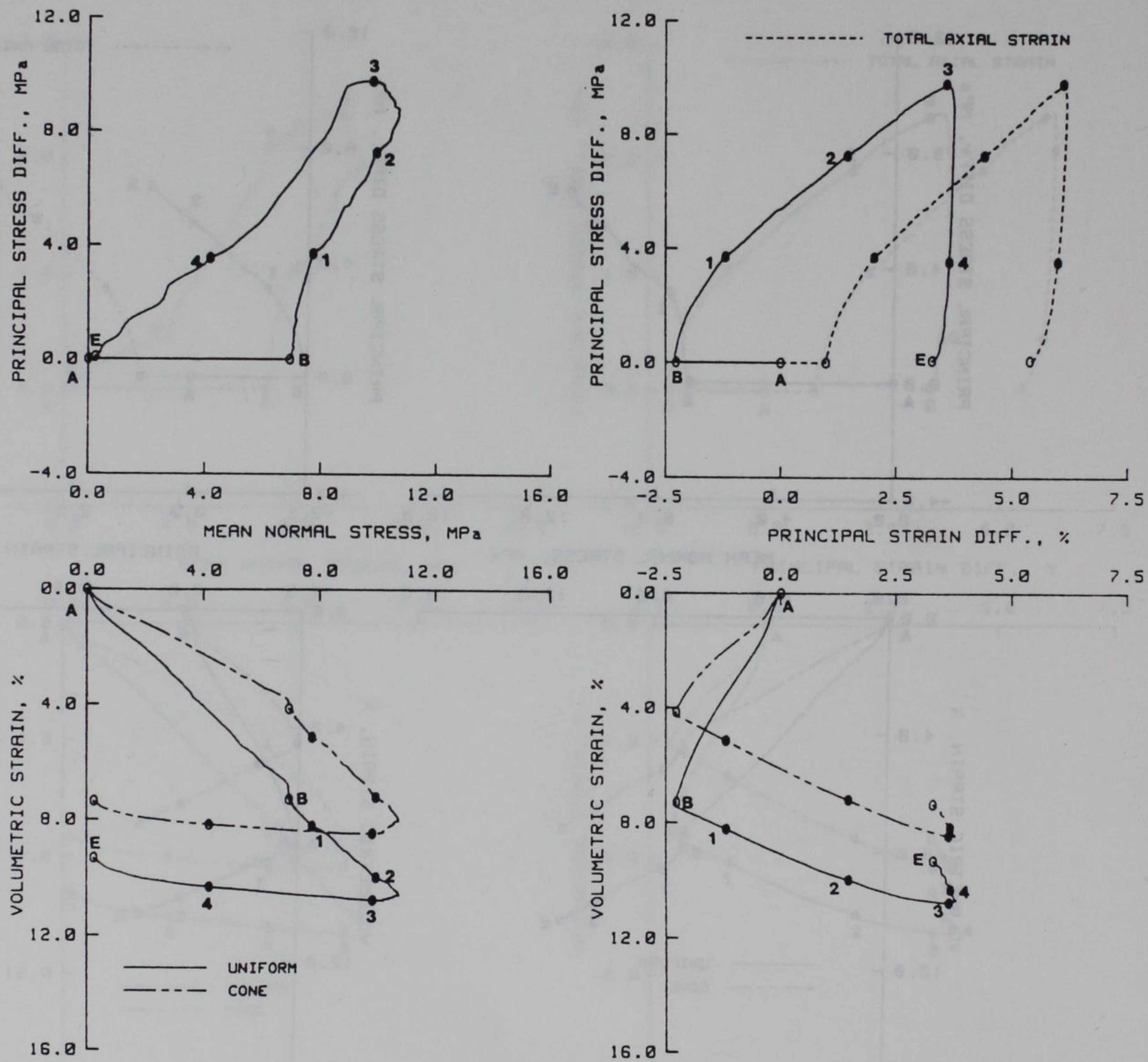


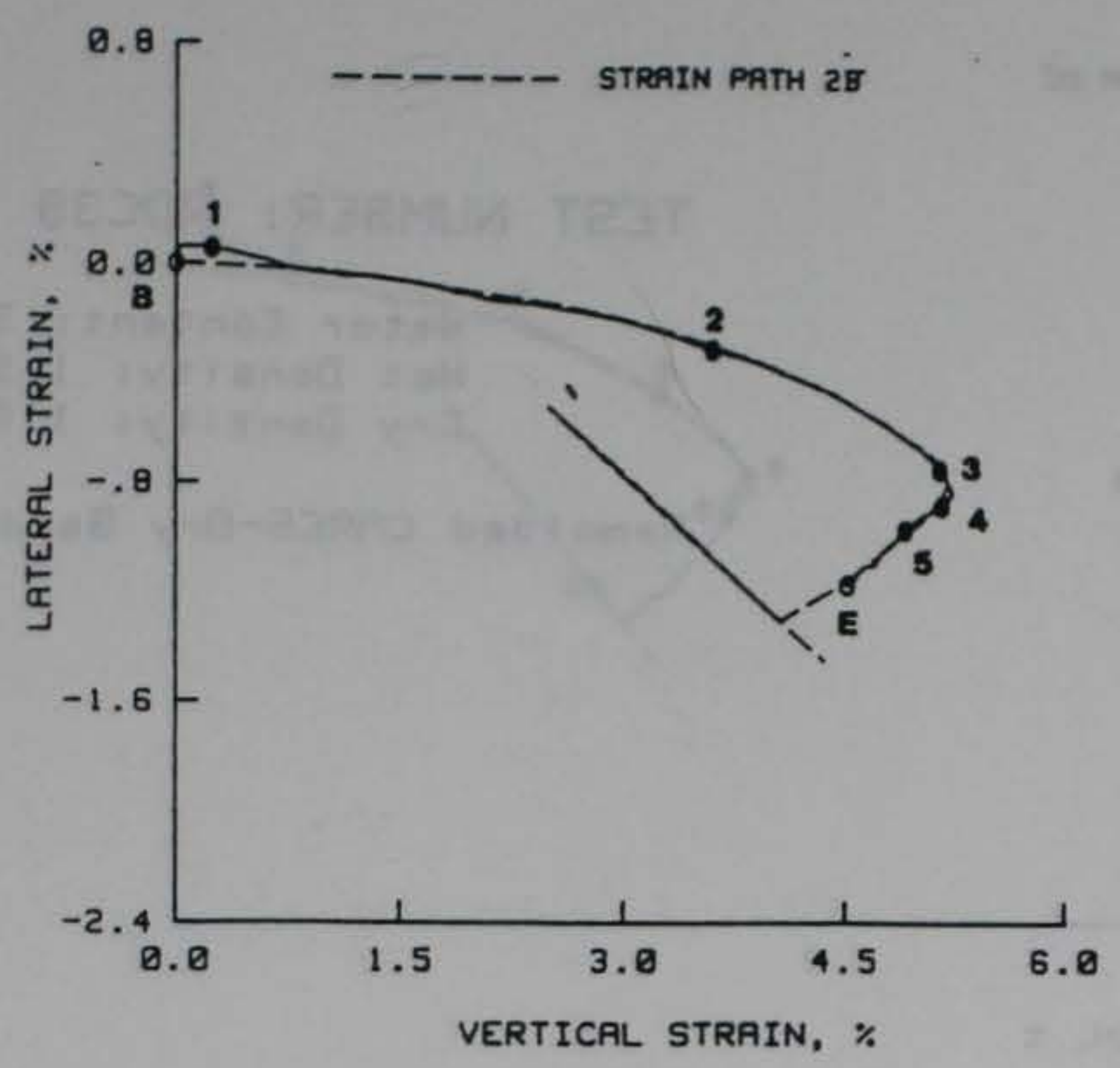

TEST NUMBER: RDC2?

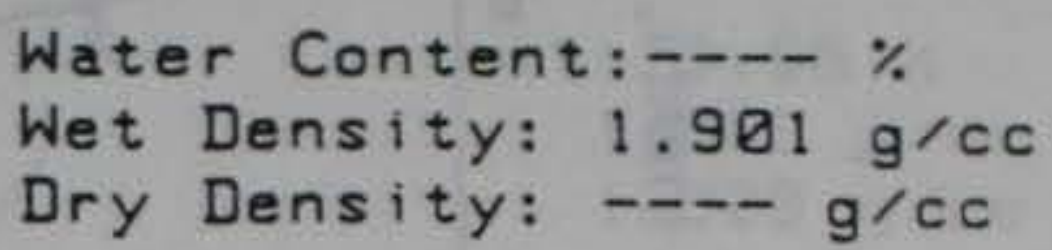

Remolded CARES-Dry Sand
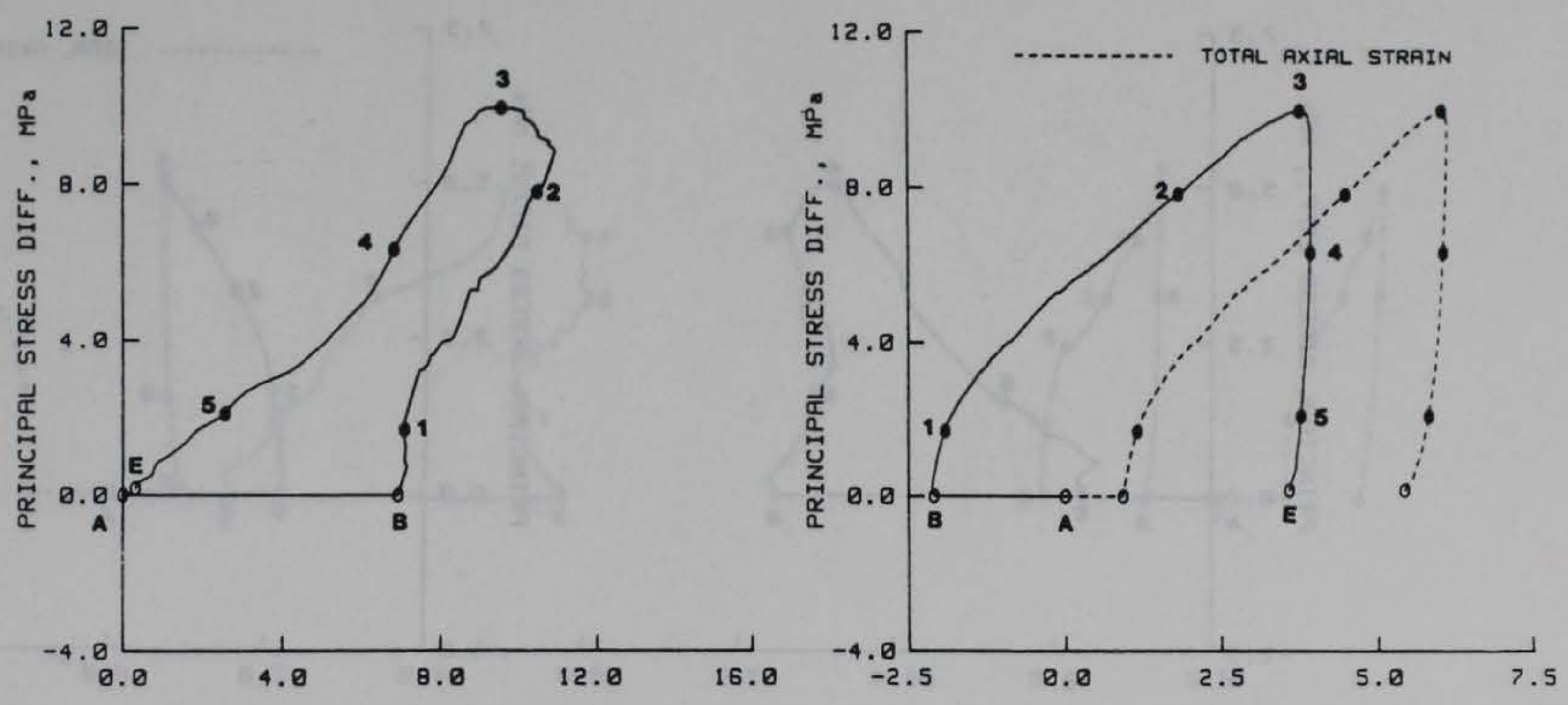

MEAN NORMAL STRESS, MPa
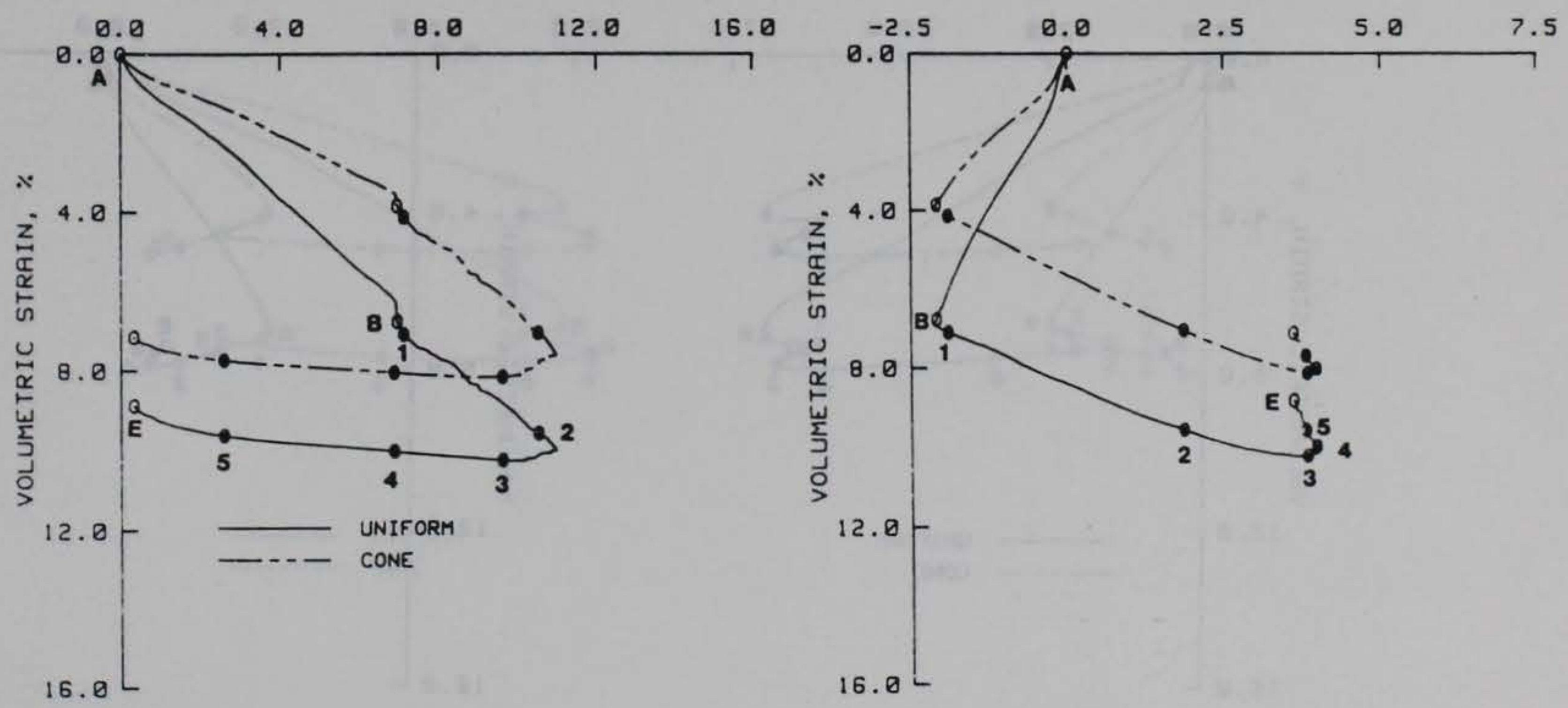


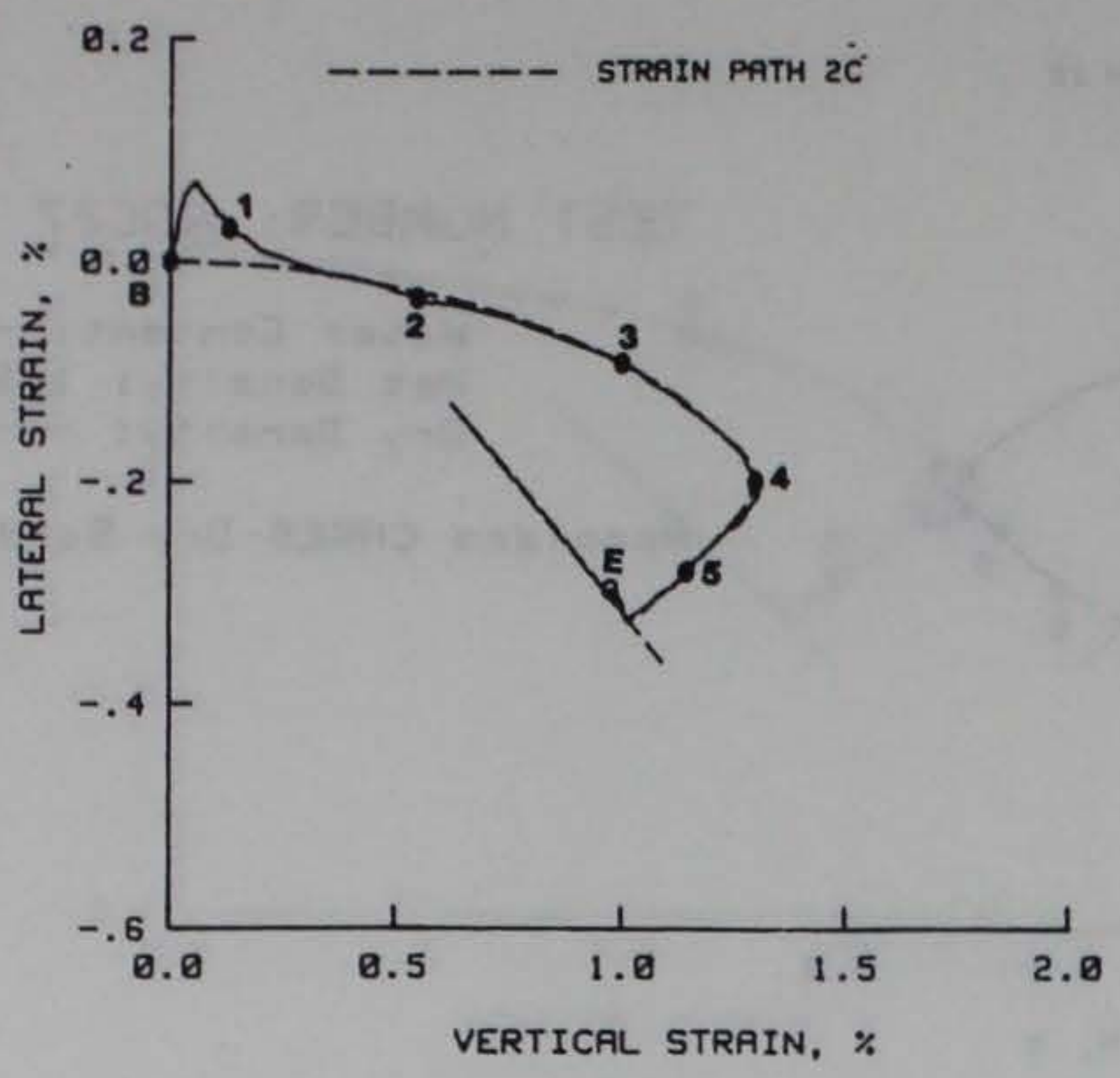

TEST NUMBER: RDC38

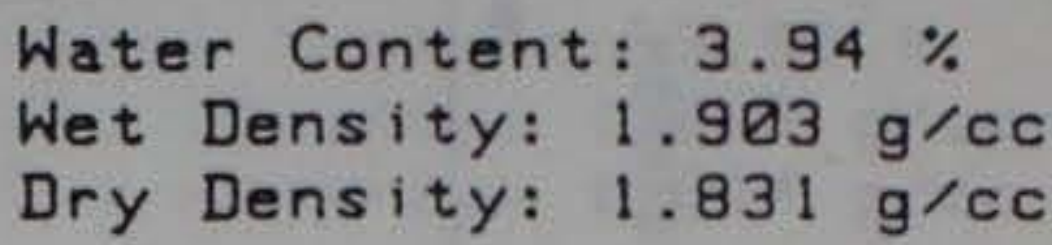

Remolded CARES-Dry Sand
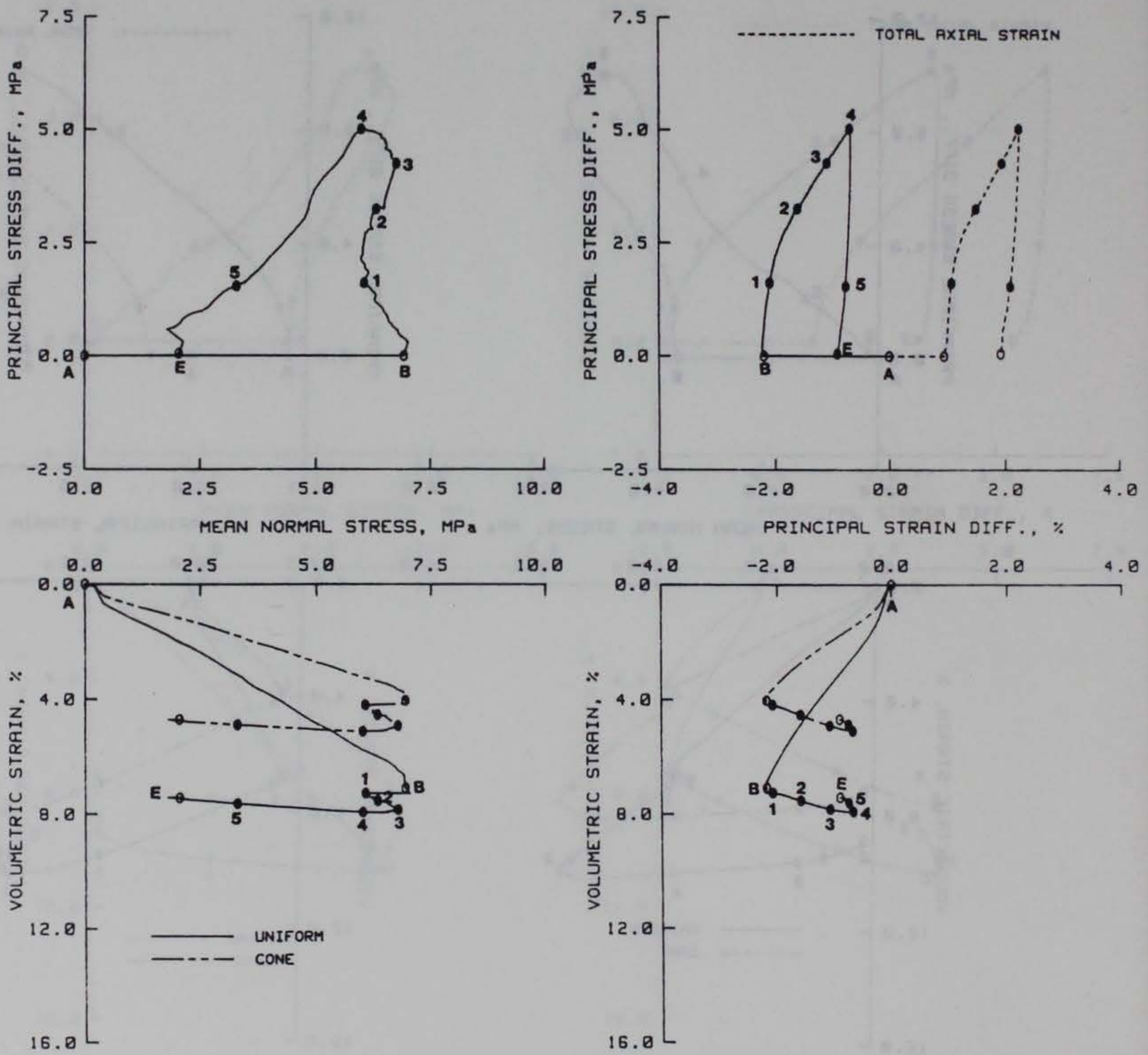


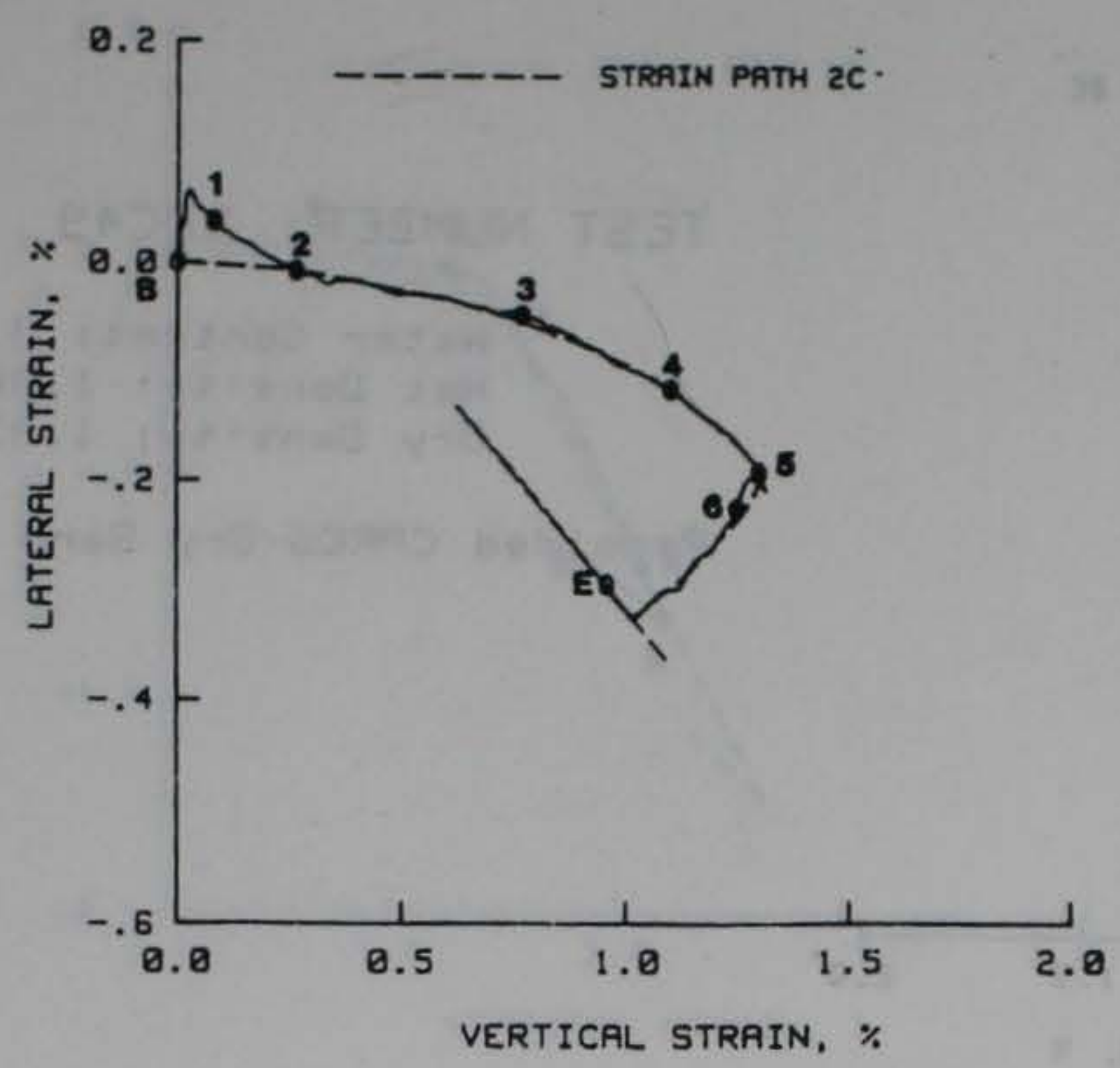

TEST NUMBER: RDC48

Water Content: $3.85 \%$

Wet Density: $1.899 \mathrm{~g} / \mathrm{cc}$

Dry Density: $1.829 \mathrm{~g} / \mathrm{cc}$

Remolded CARES-Dry Sand
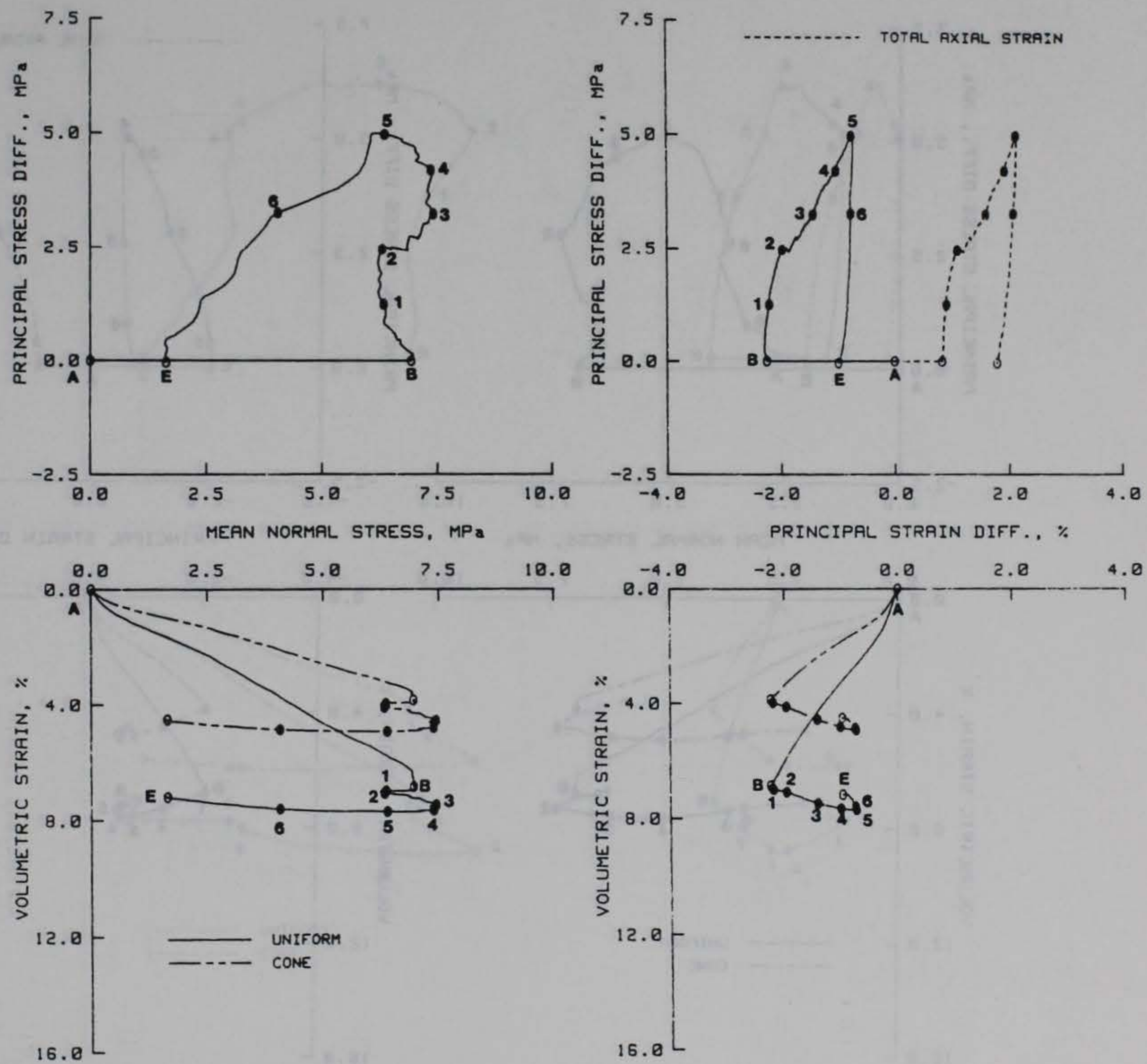


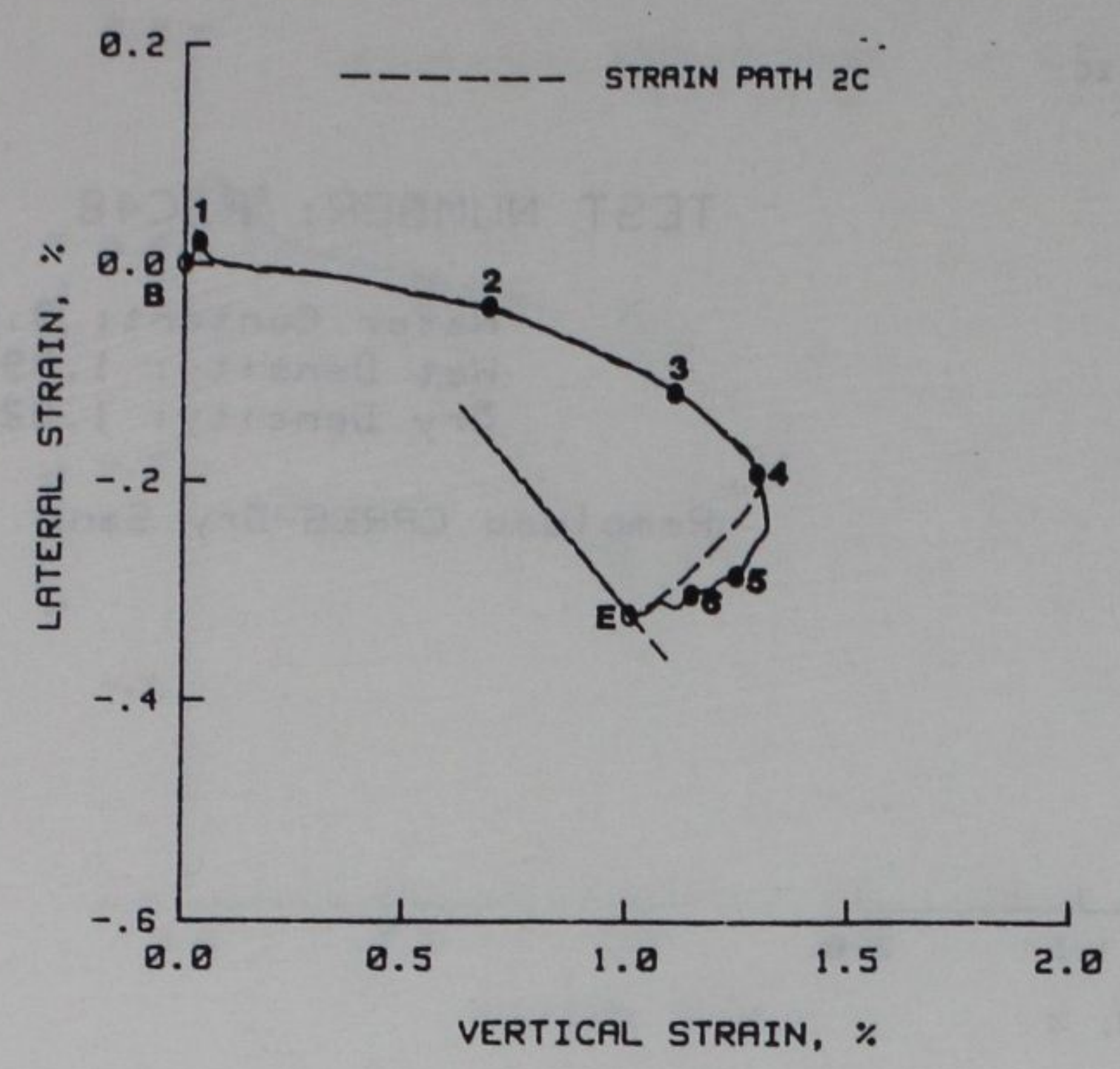

TEST NUMBER: RDC49

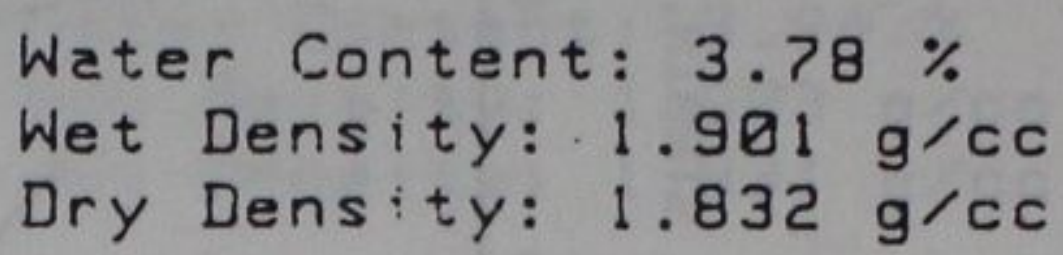

Remolded CARES-Dry Sand
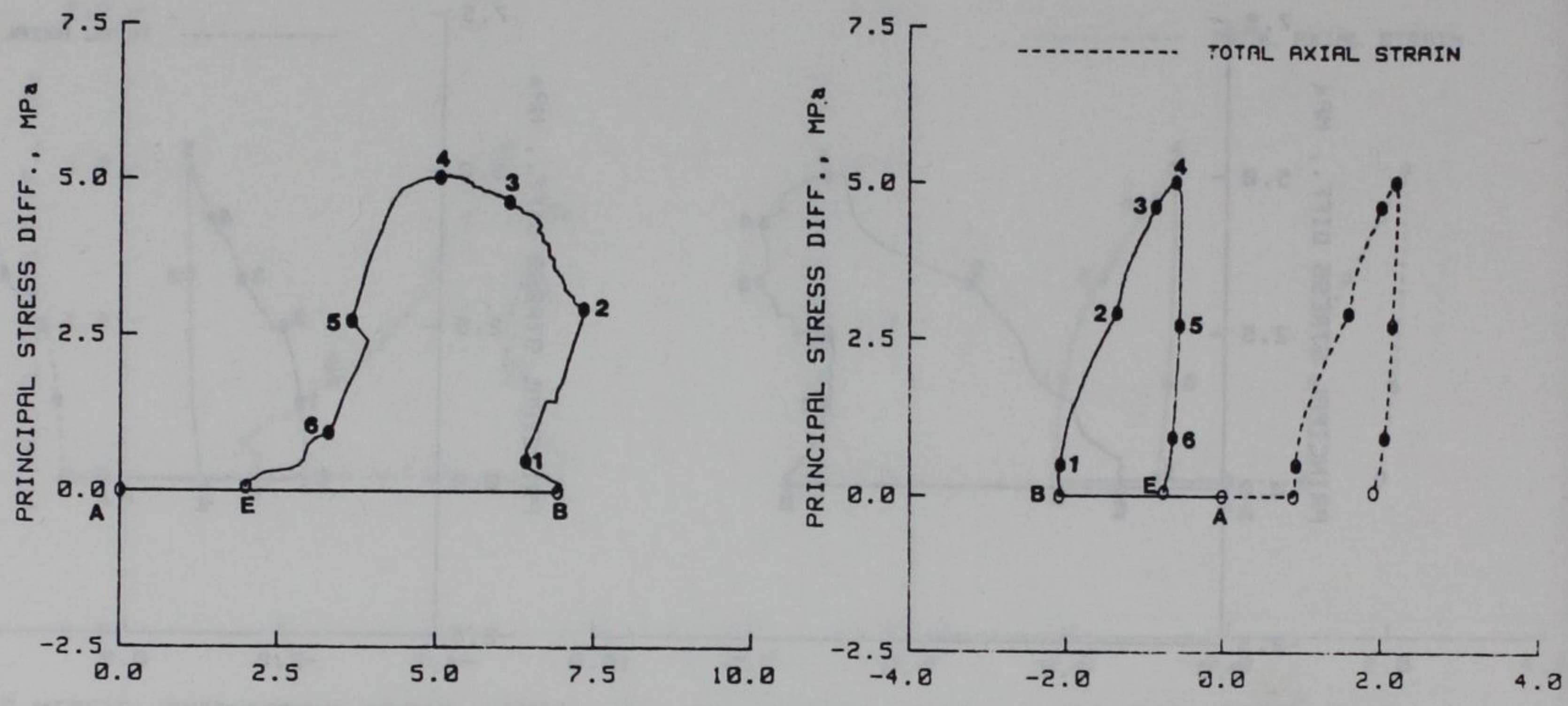

MEAN NORMAL STRESS, MPa
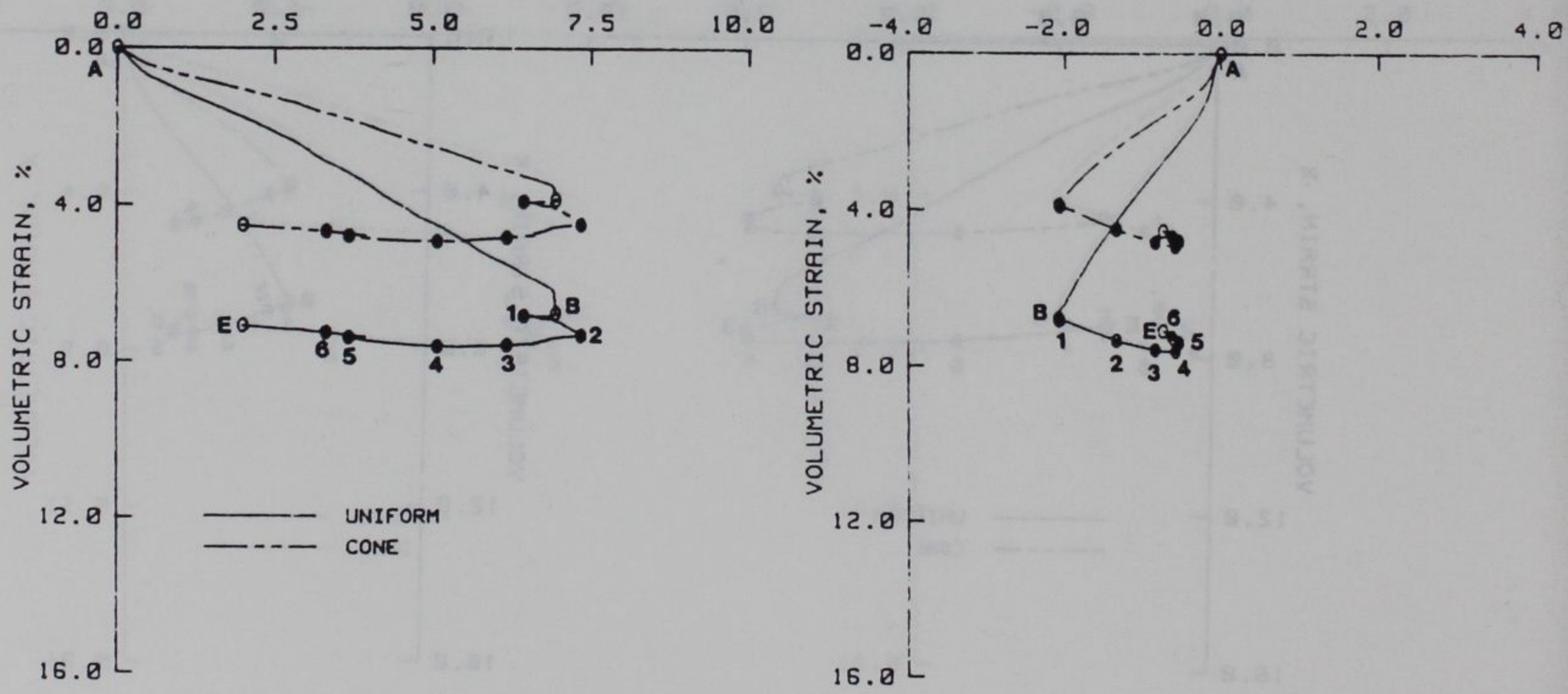


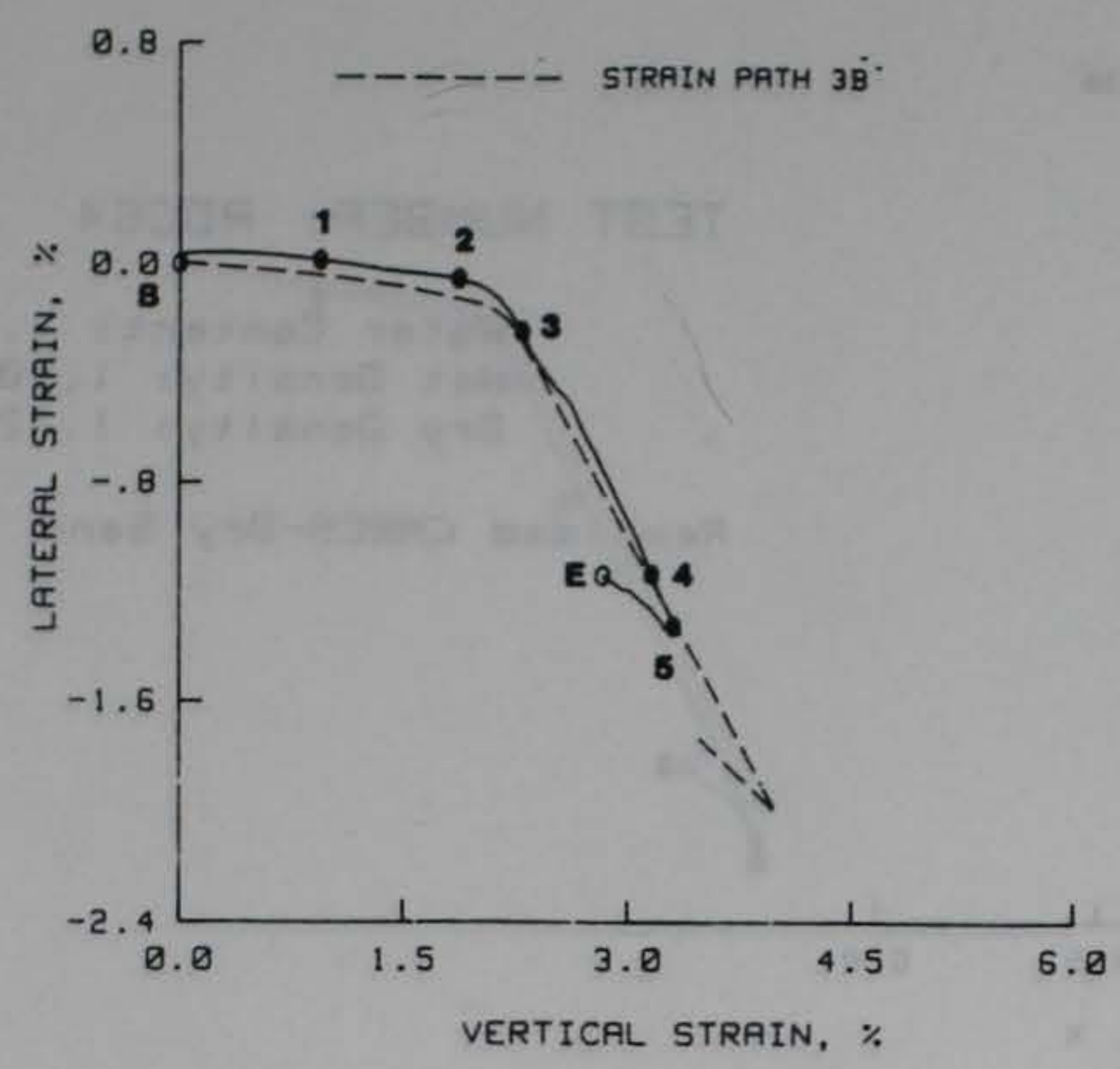

TEST NUMBER: RDC63

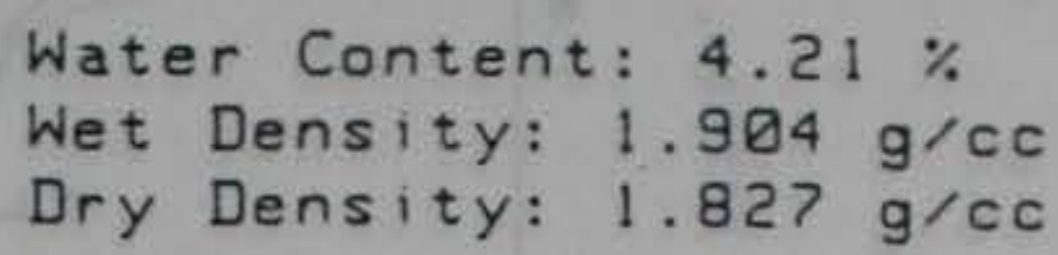

Remolded CARES-Dry Sand
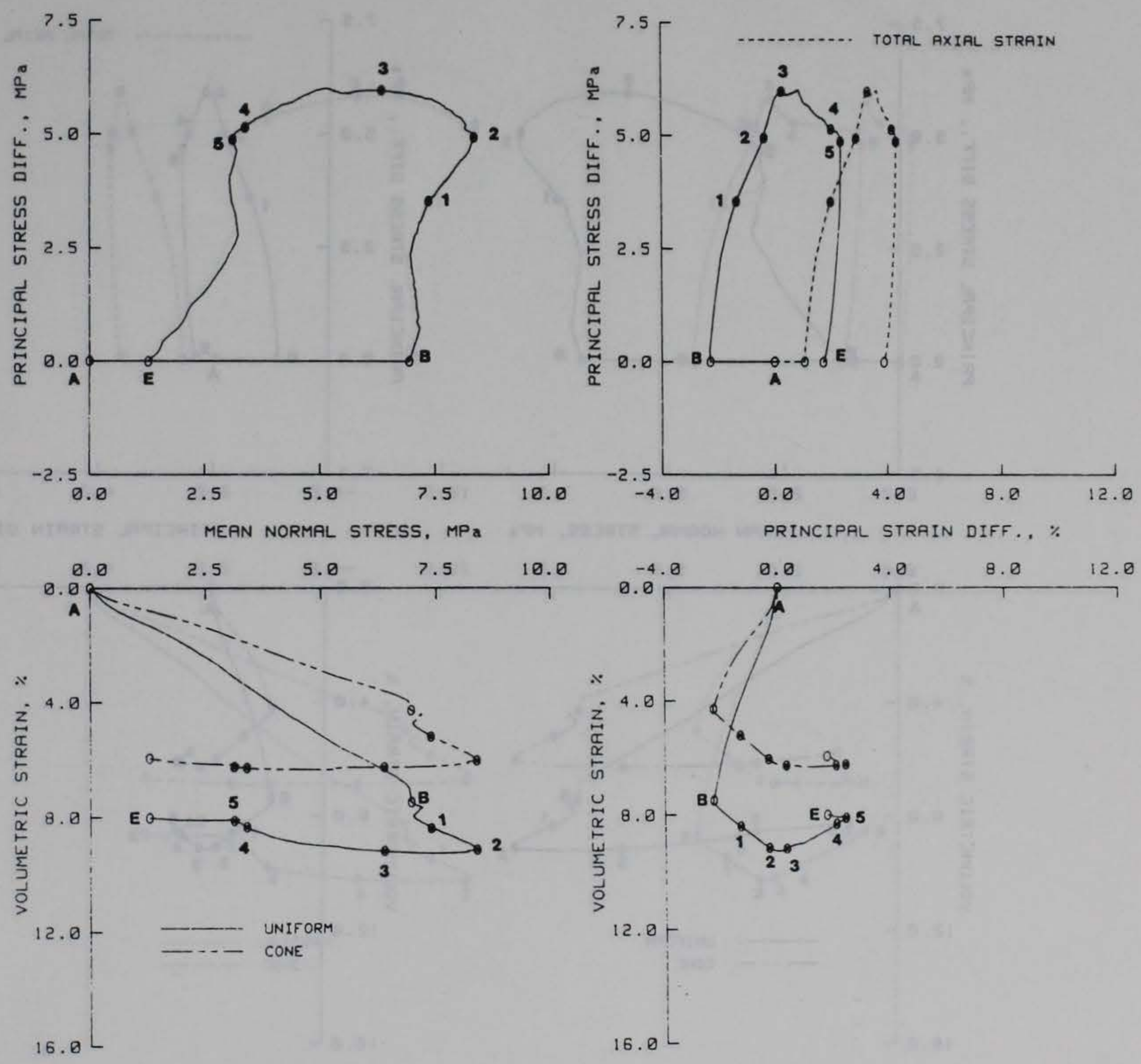


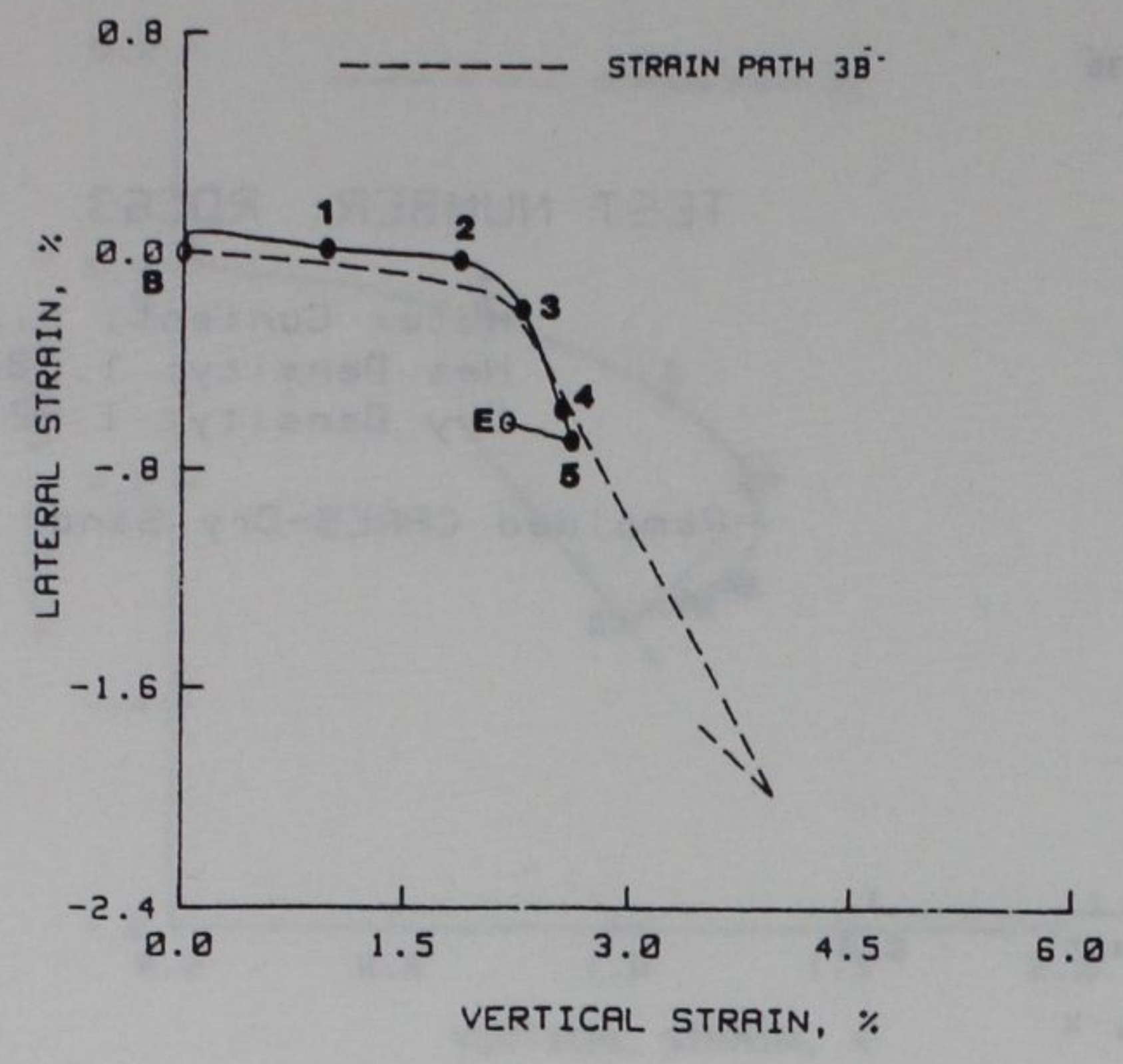

TEST NUMBER: RDC64

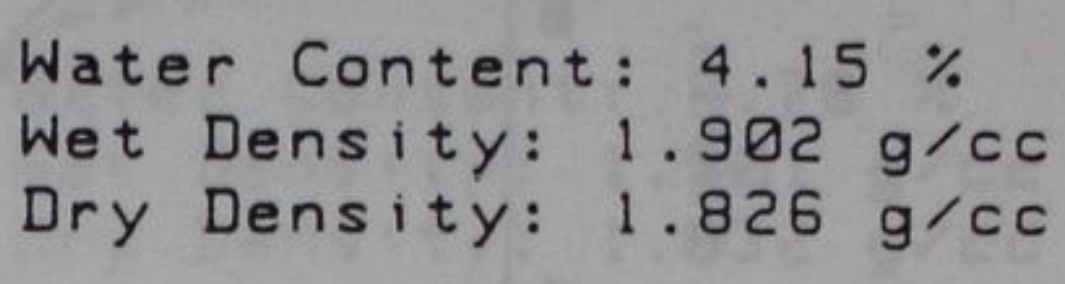

Remolded CARES-Dry Sand
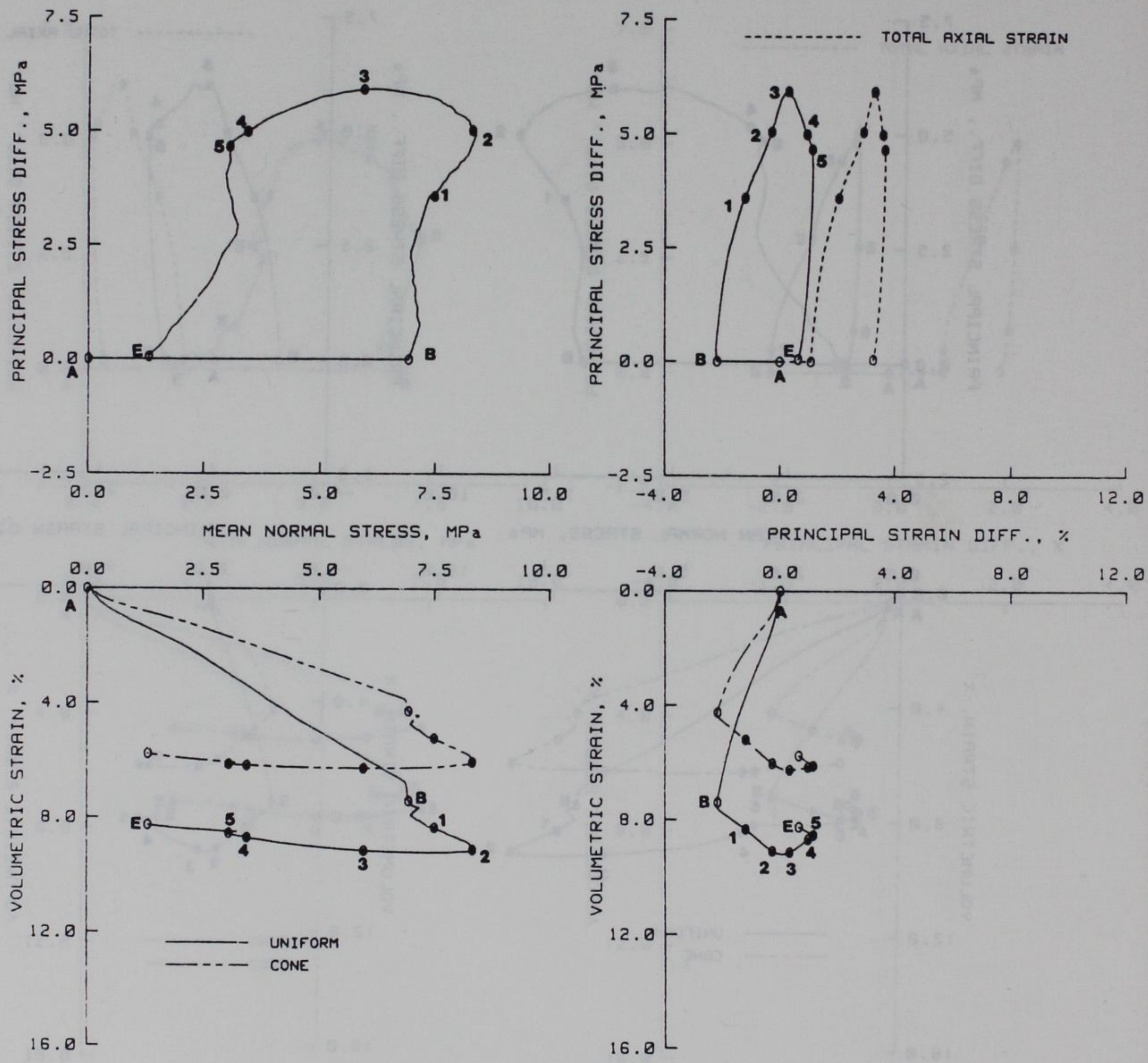


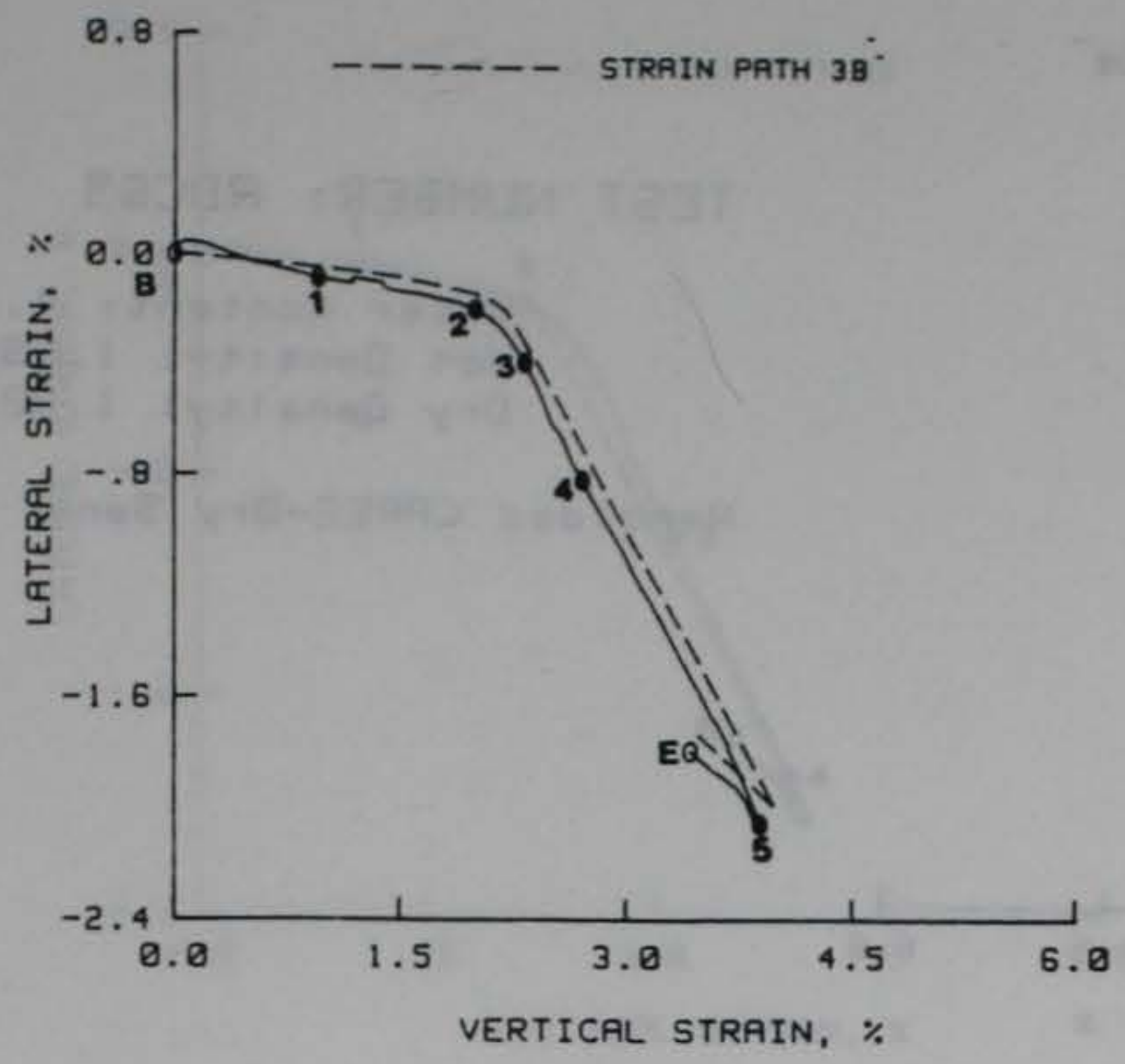

TEST NUMBER: RDCG7

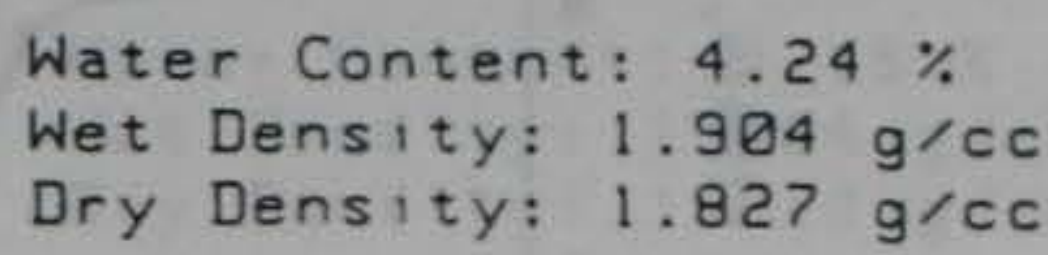

Remolded CARES-Dry Sand
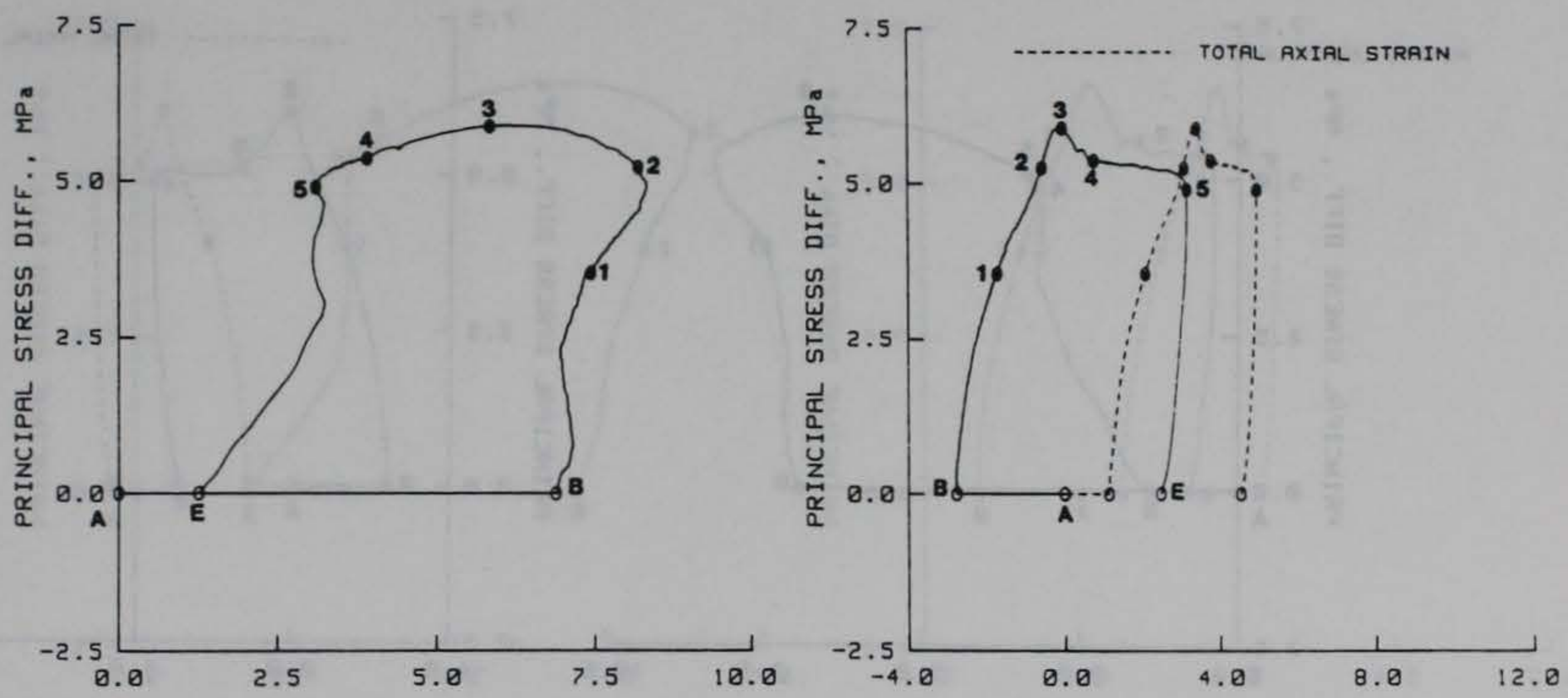

MEAN NORMAL STRESS, MPa
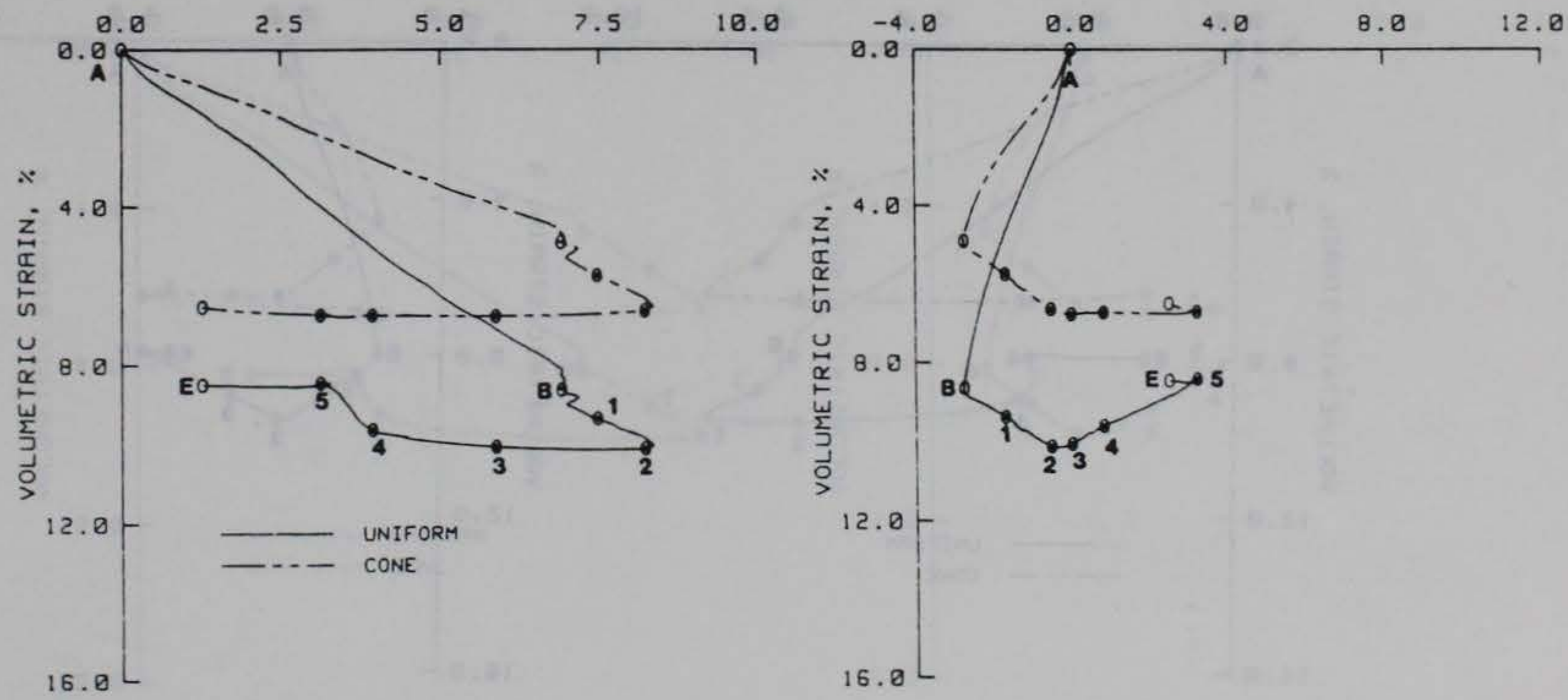


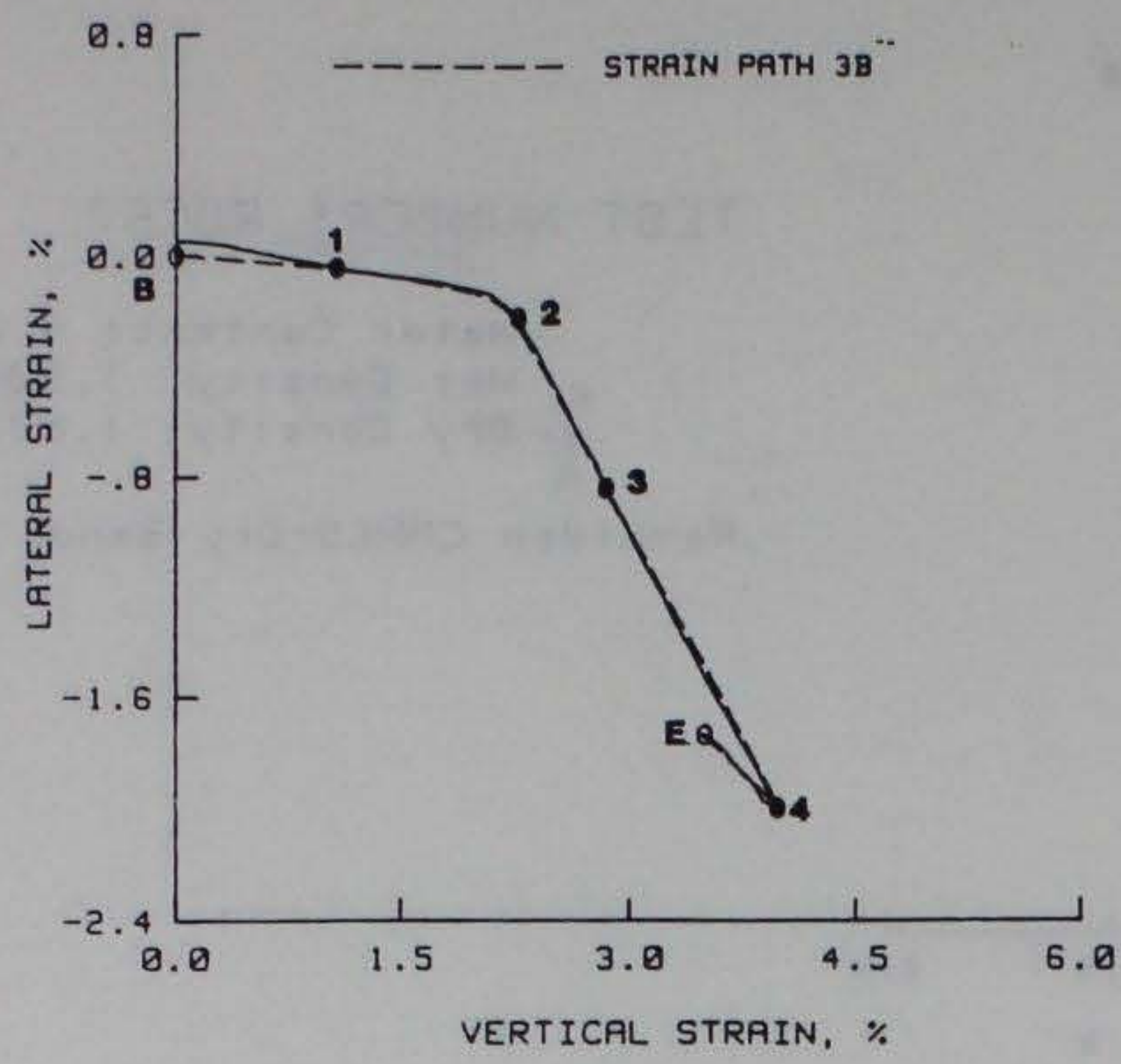

TEST NUMBER: RDC65

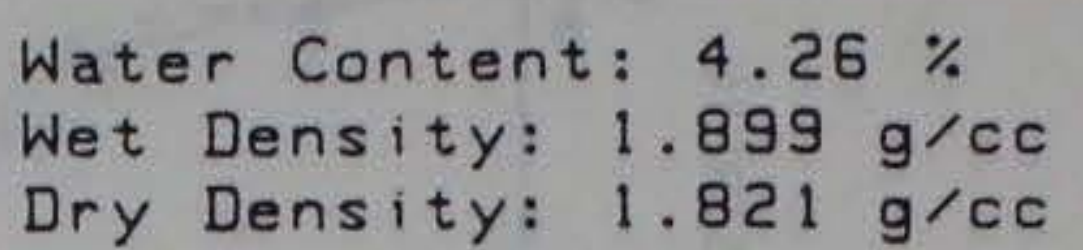

Remolded CARES-Dry Sand
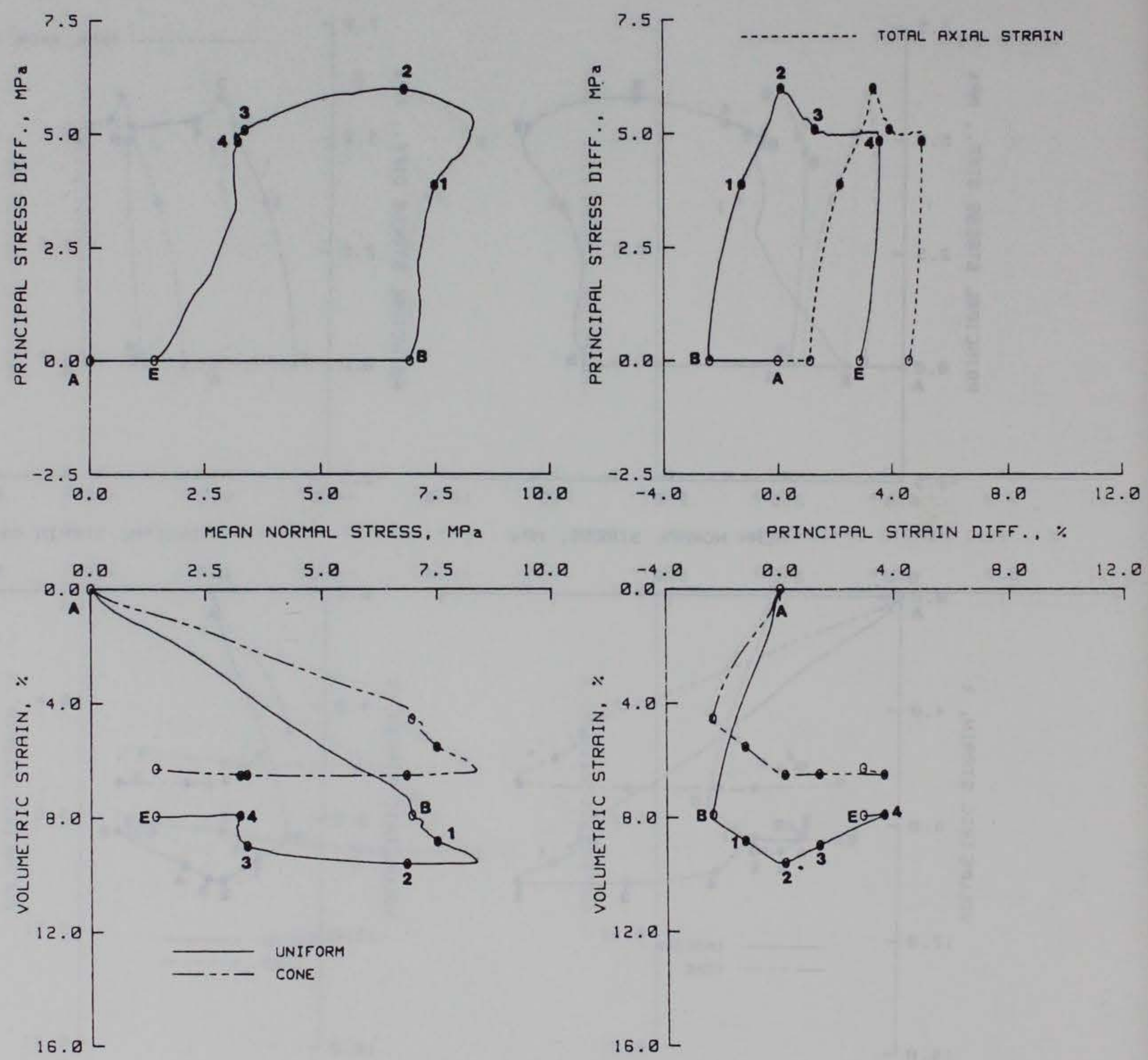


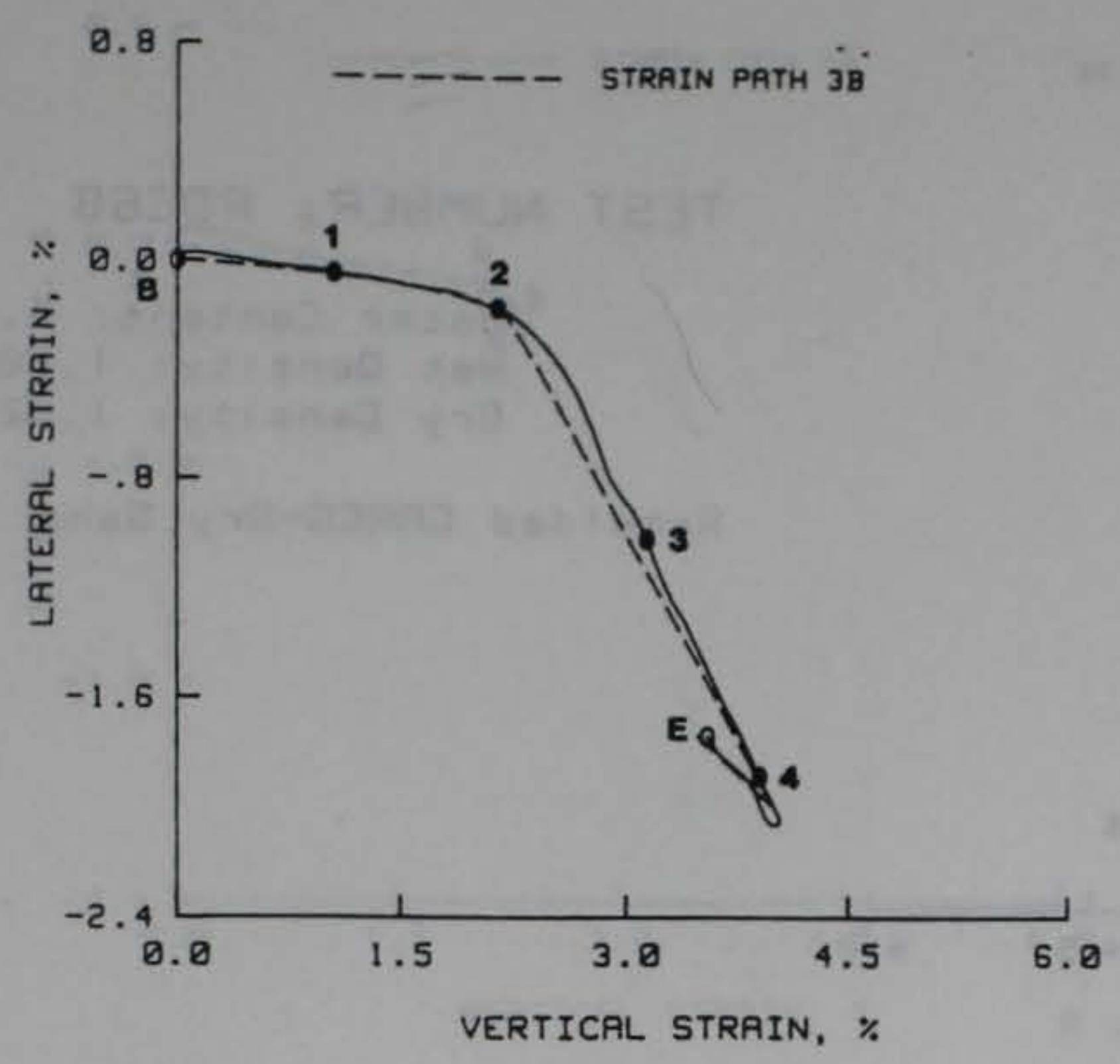

TEST NUMBER: RDCG6

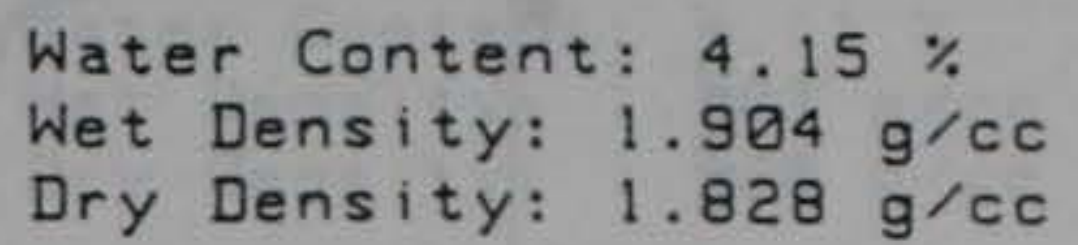

Remolded CARES-Dry Sand
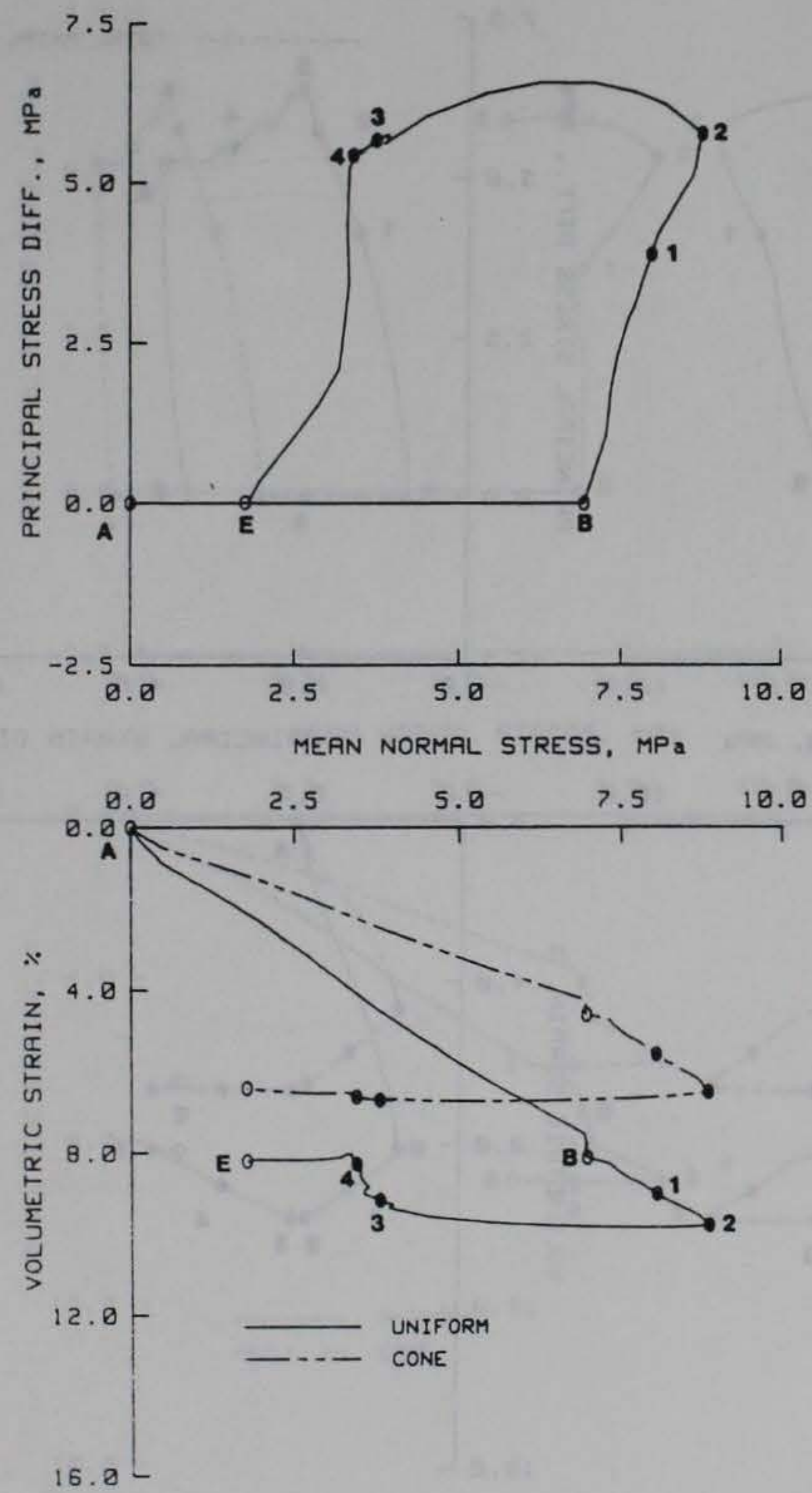
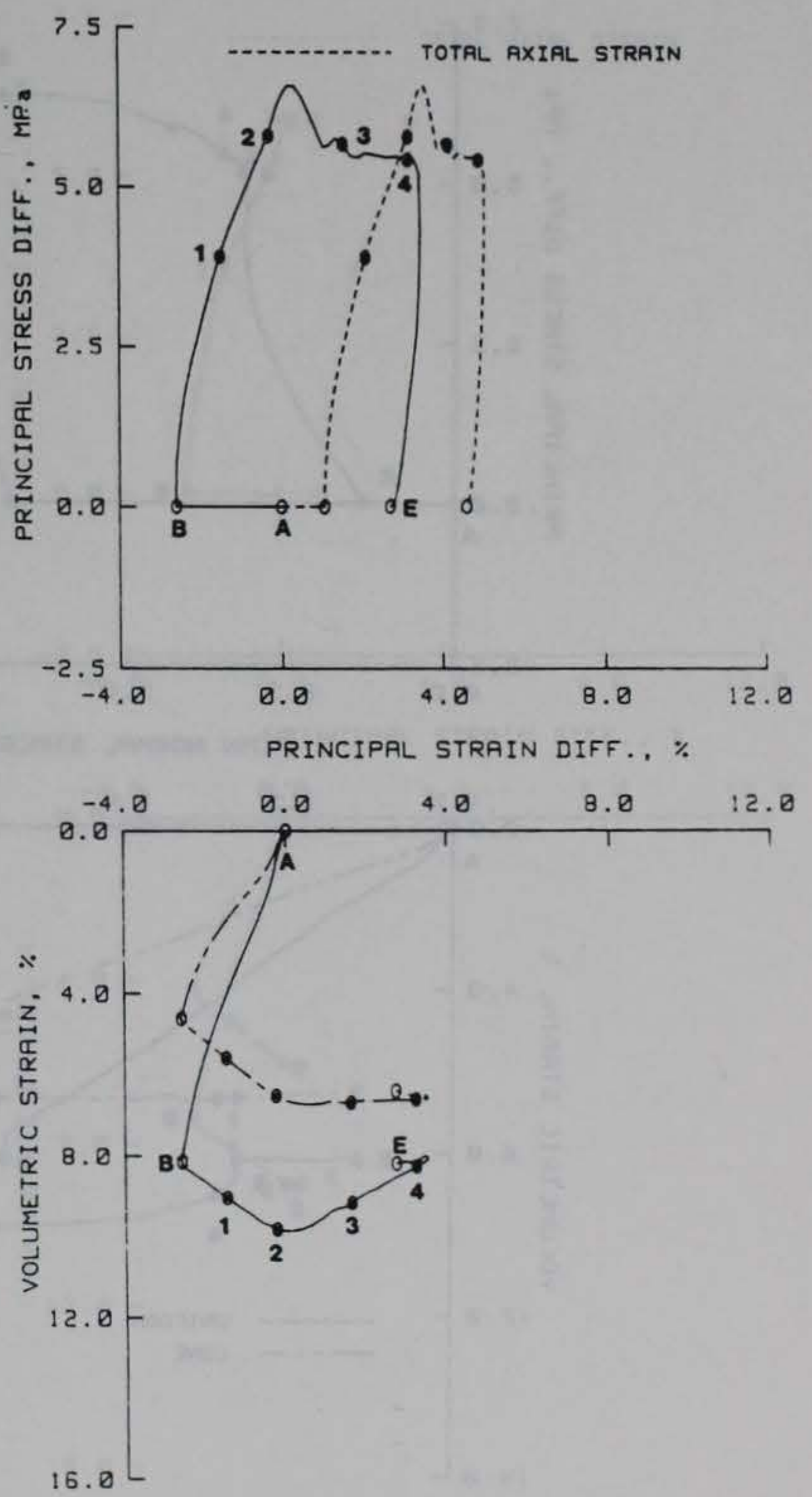


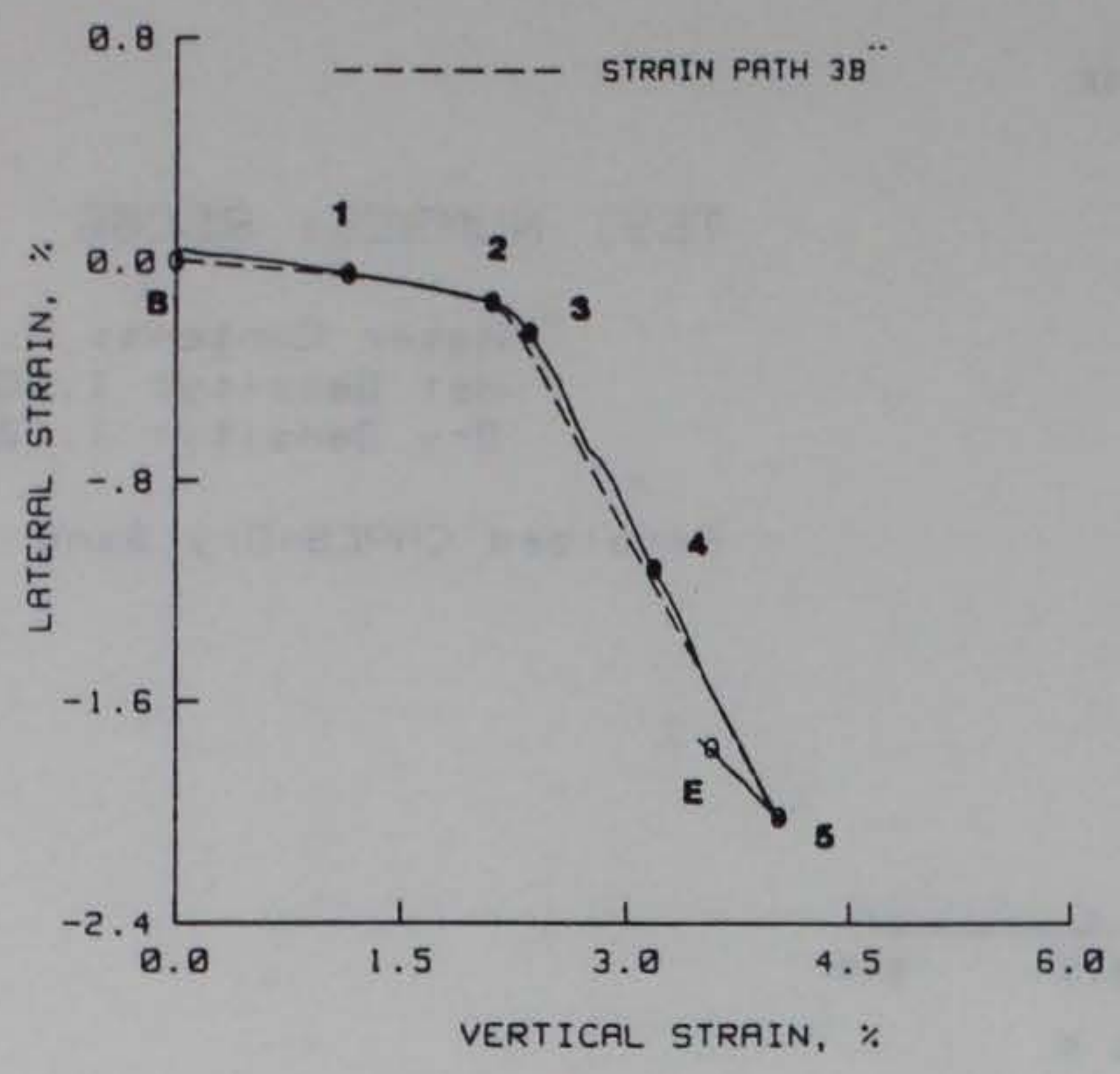

TEST NUMBER: RDC68

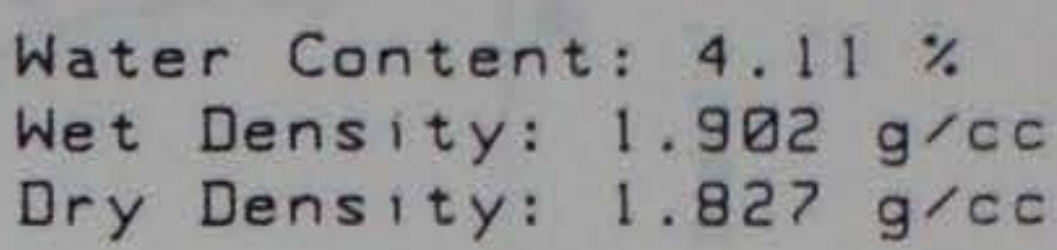

Remolded CARES-Dry S and
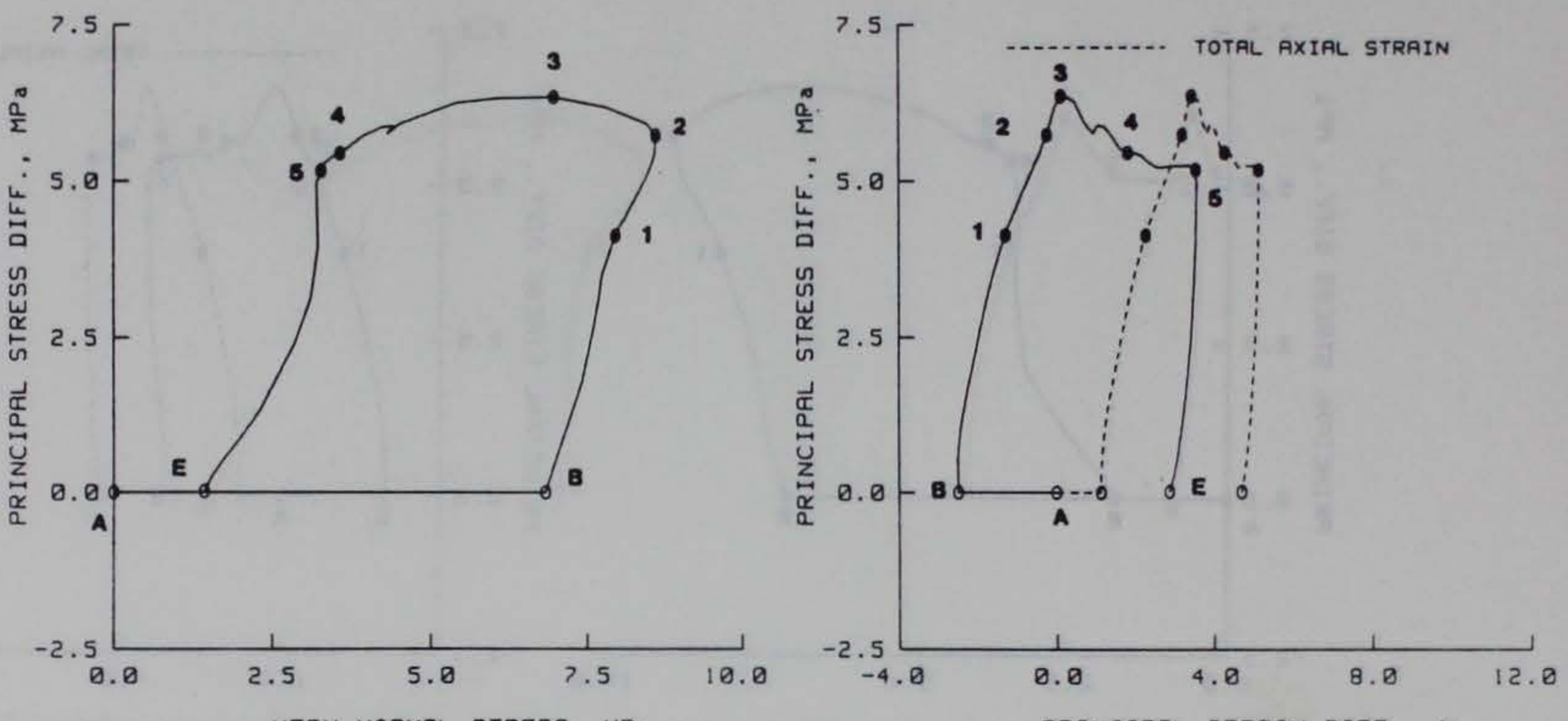

MEAN NORMAL STRESS, MPa
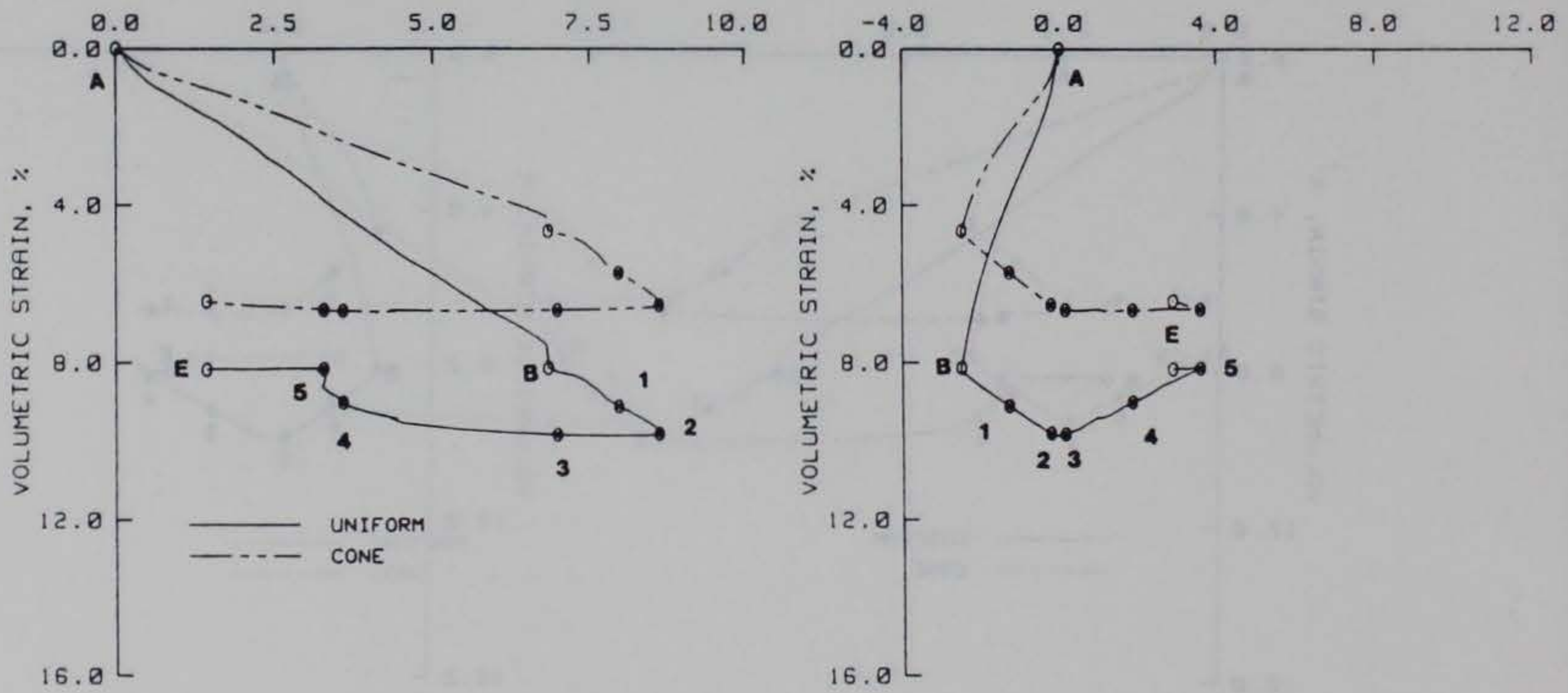


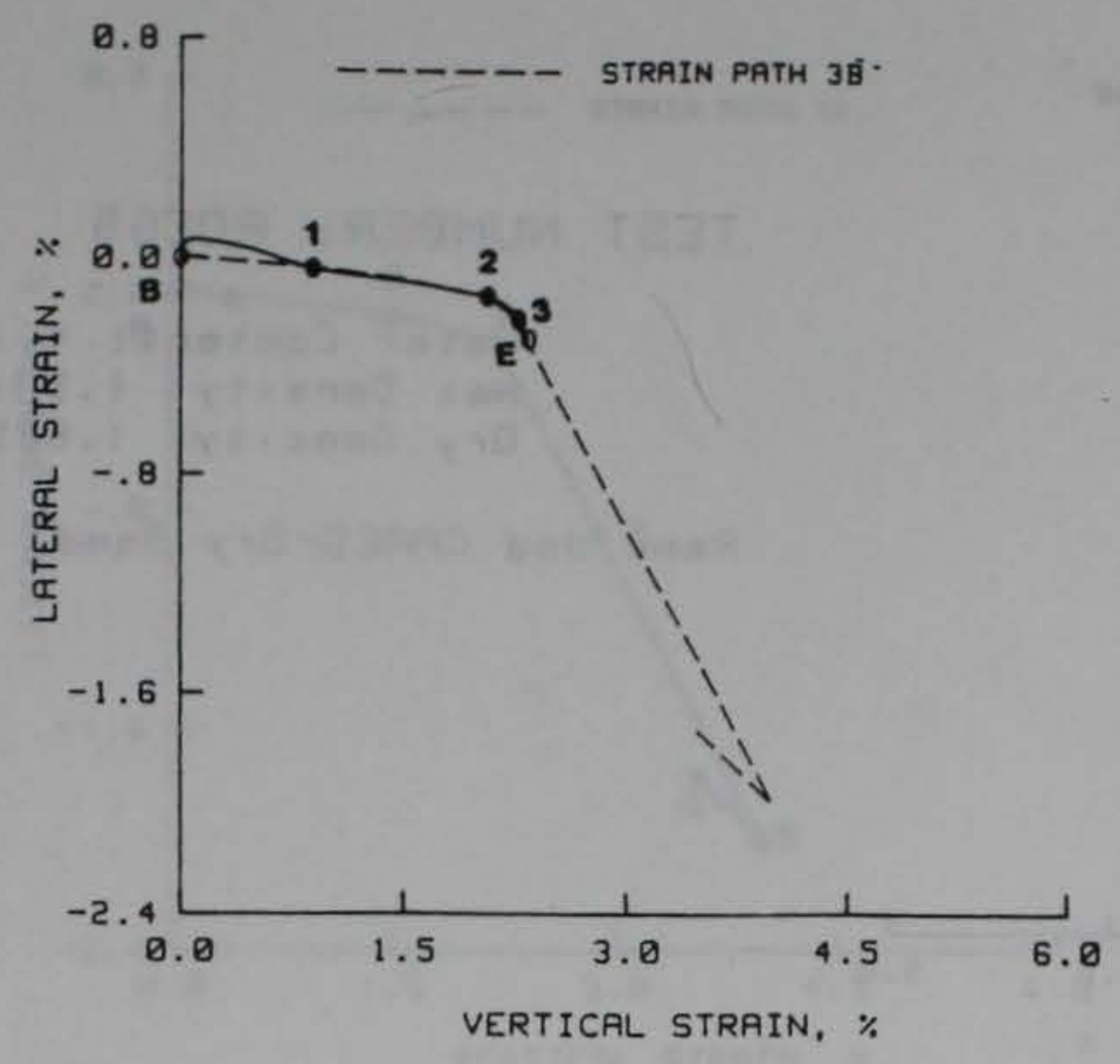

TEST NUMBER: RDC>2

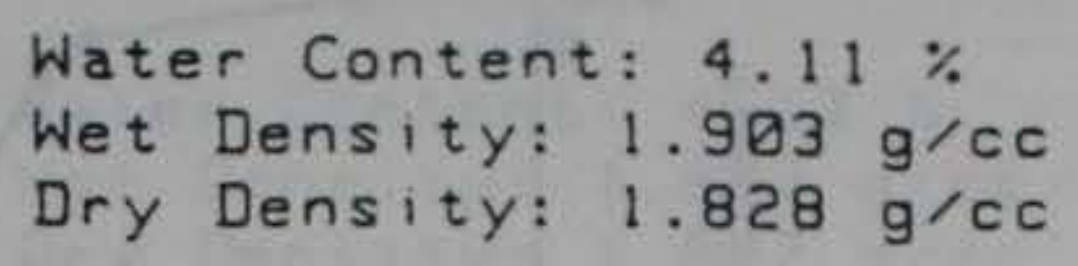

Remolded CARES-Dry Sand
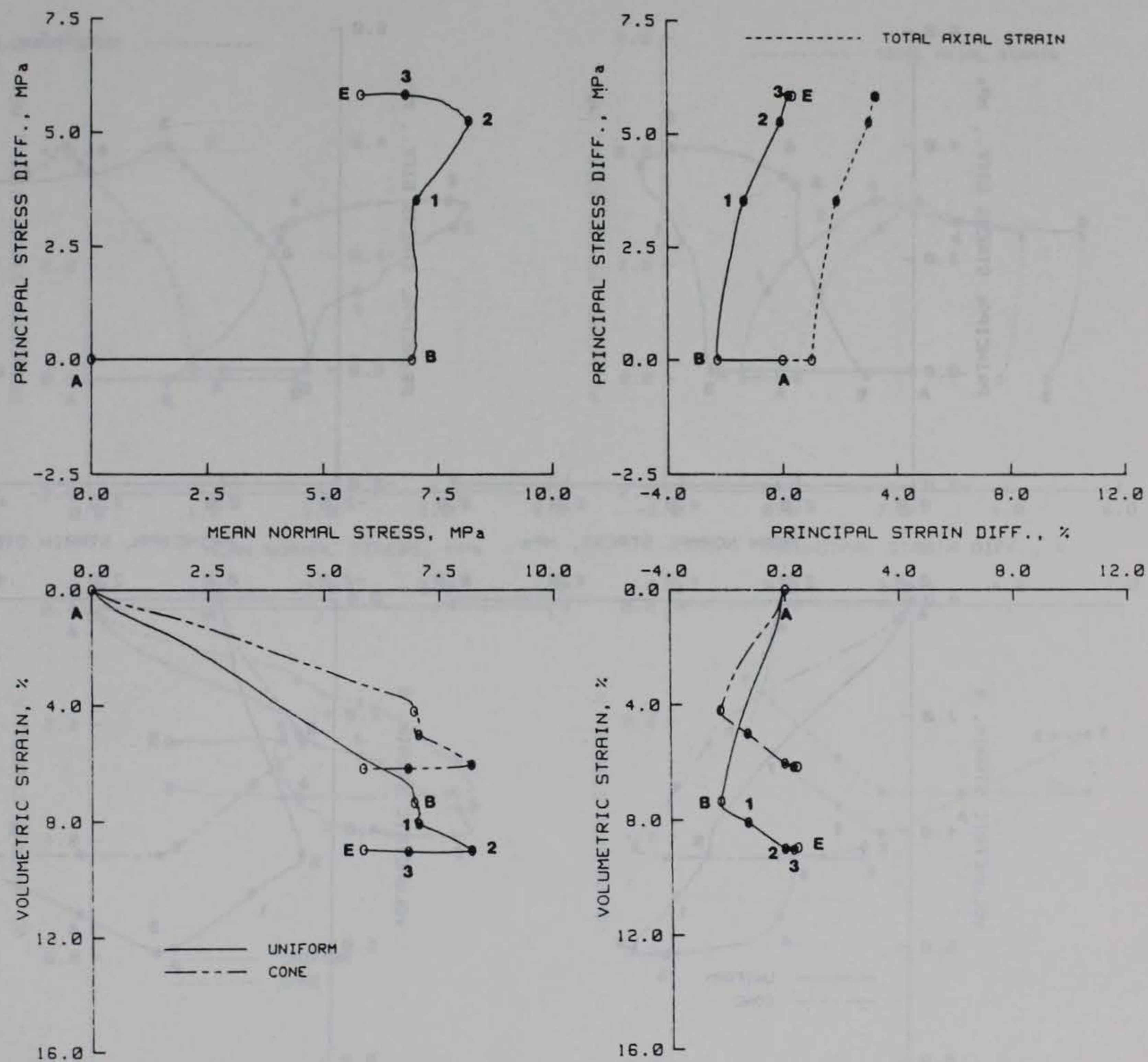


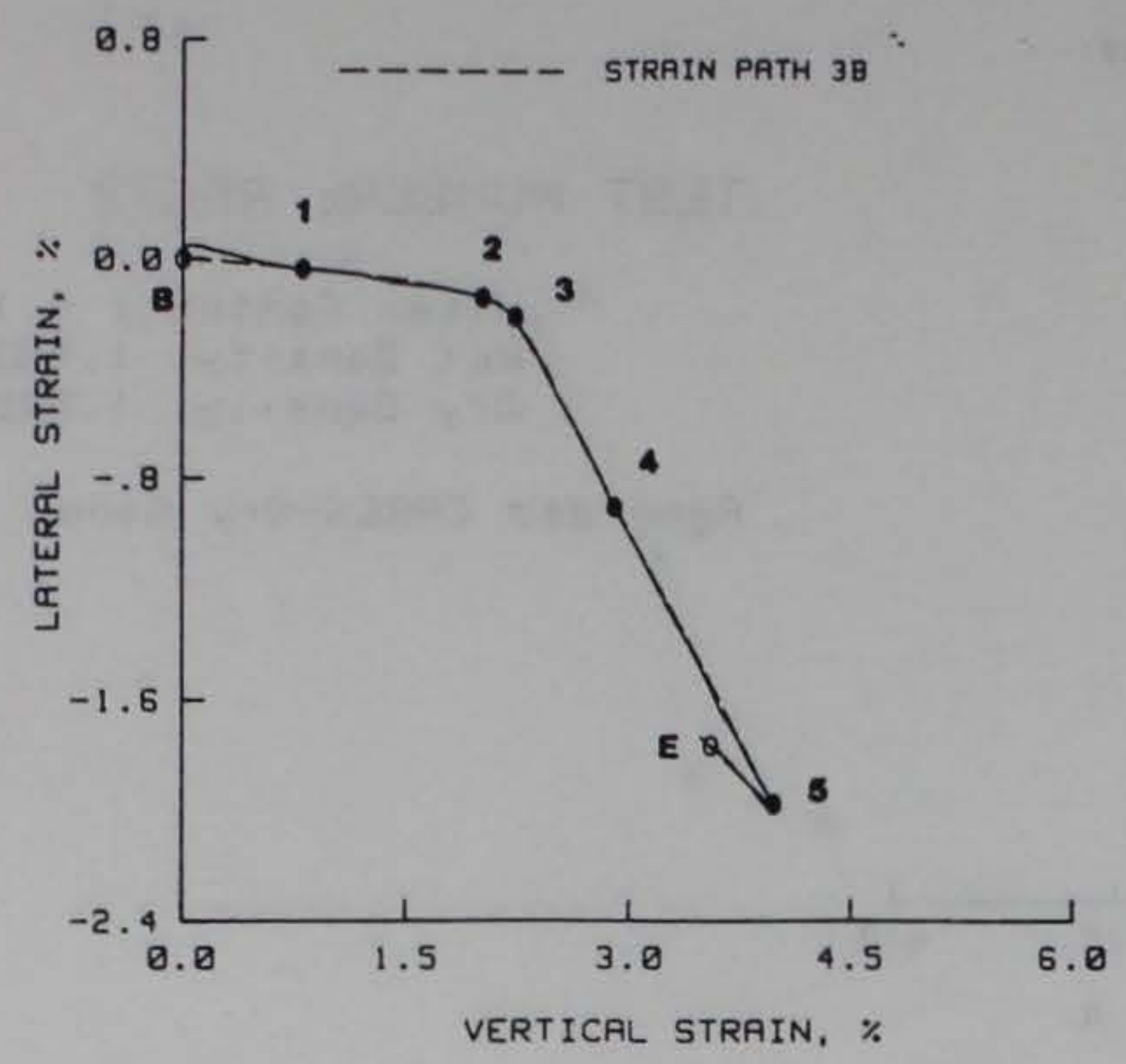

TEST NUMBER: RDC69

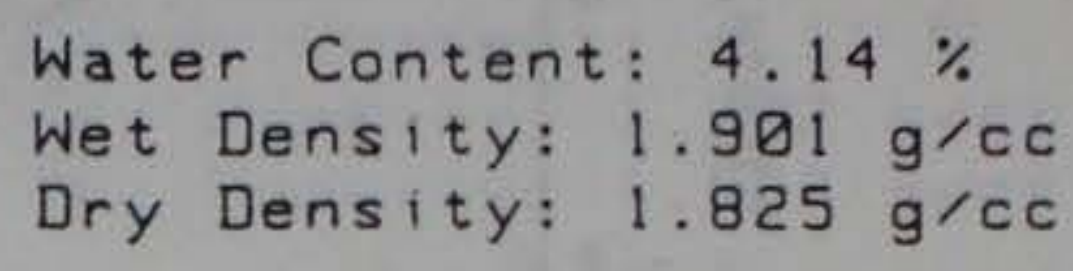

Remolded CARES-Dry Sand
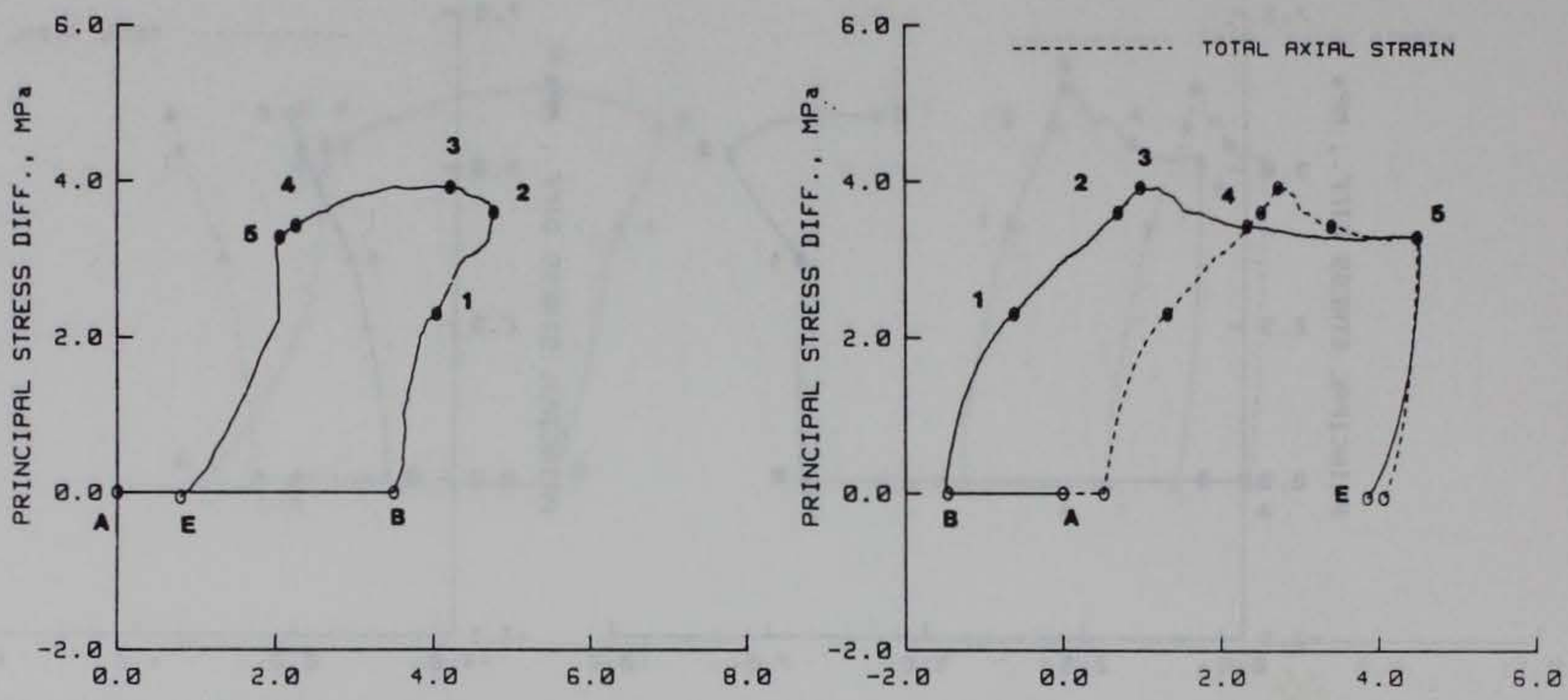

MEAN NORMAL STRESS, MPa
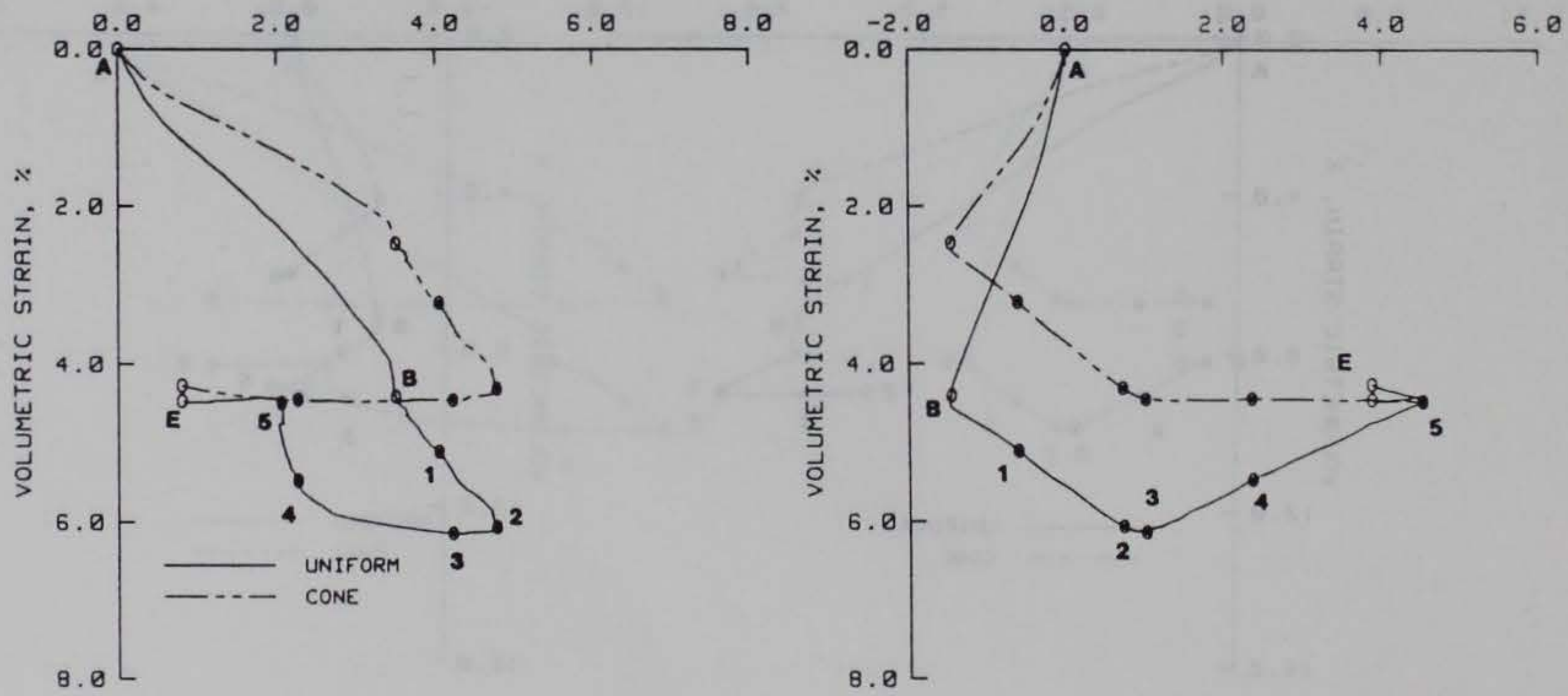


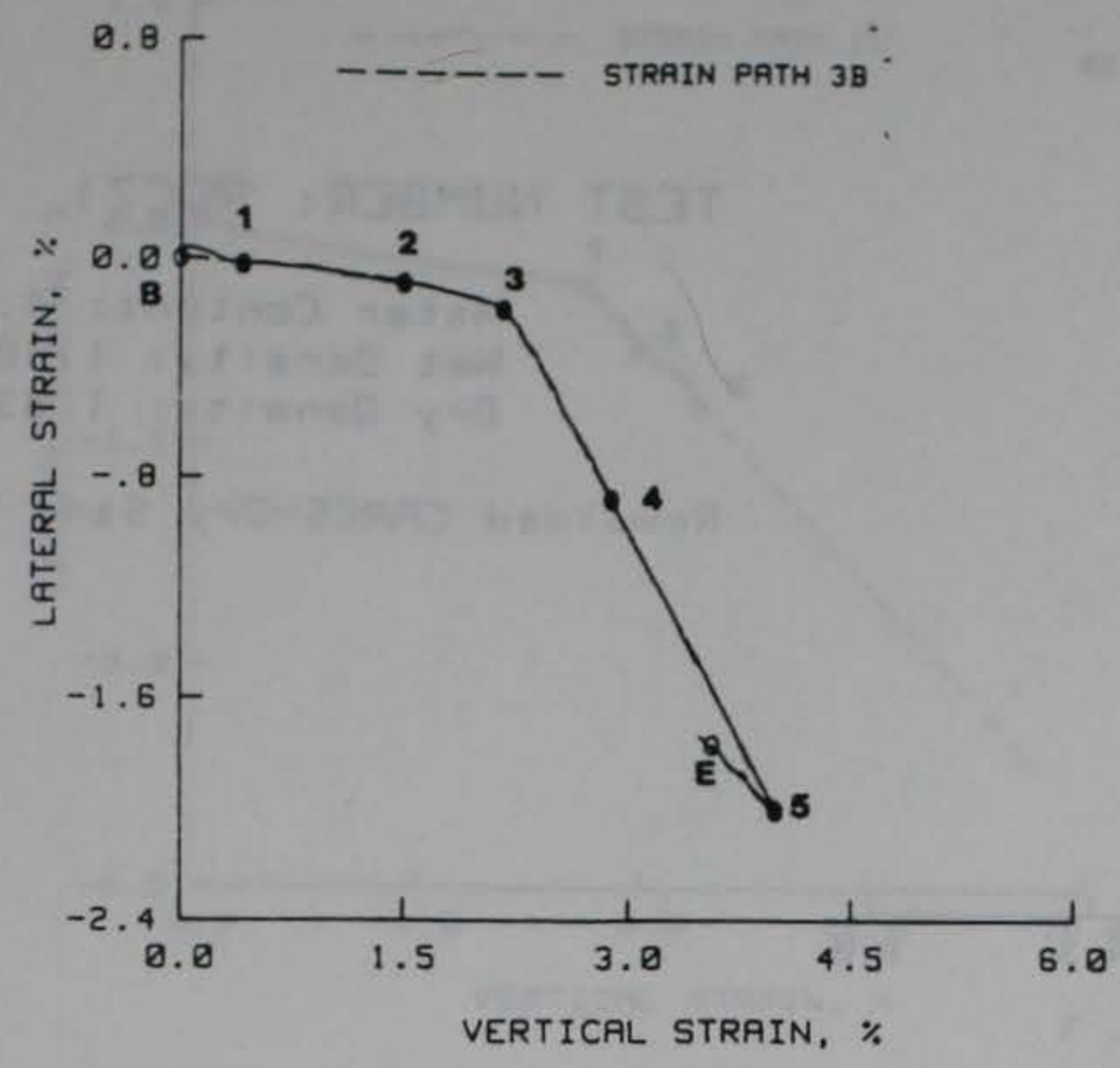

TEST NUMBER: RDCPD

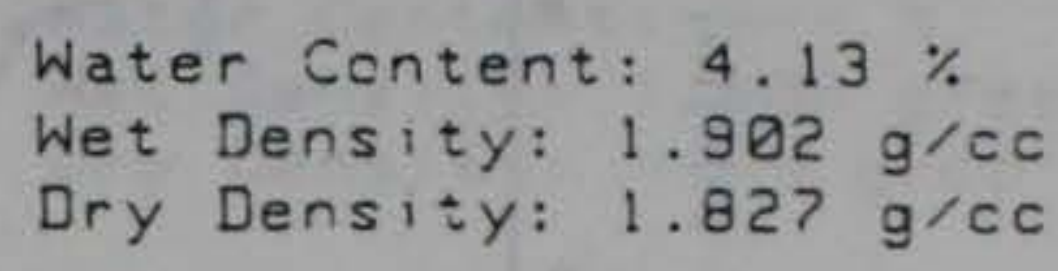

Remolded CARES-Dry Sand
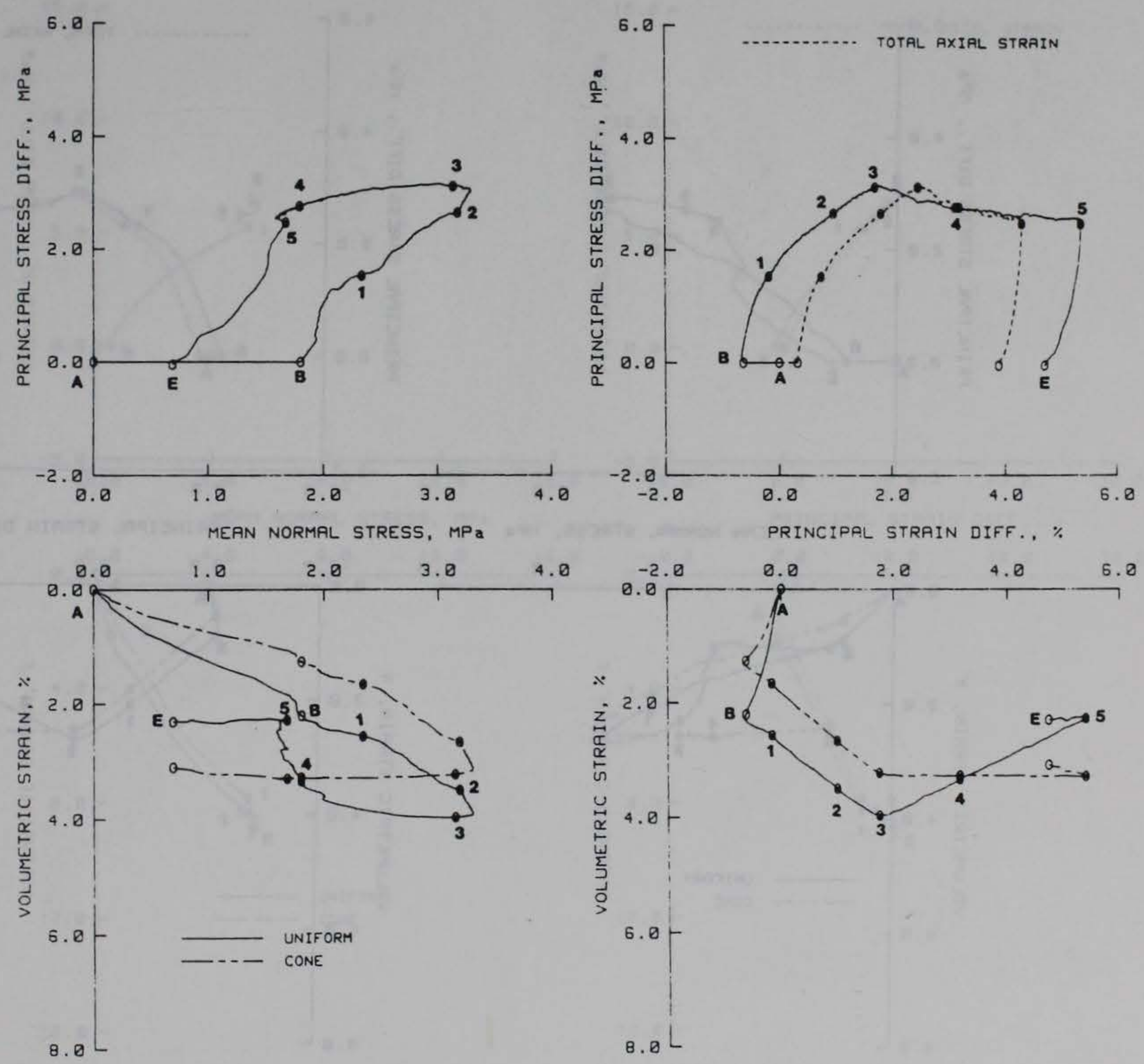


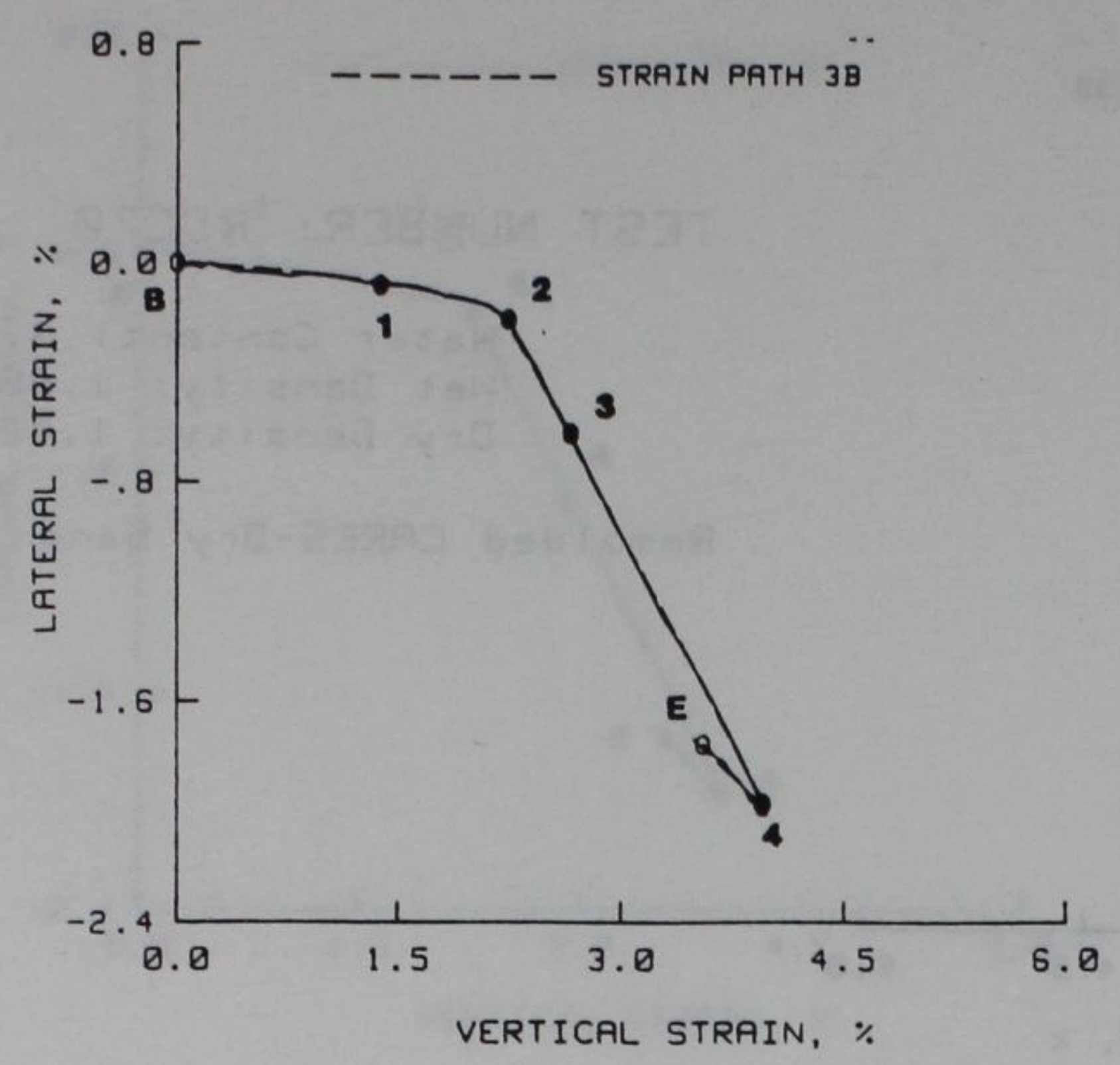

TEST NUMBER: RDC> 1

Water Content: $4.13 \%$

Wet Density: $1.906 \mathrm{~g} / \mathrm{cc}$

Dry Density: $1.830 \mathrm{~g} / \mathrm{cc}$

Remolded CARES-Dry Sand
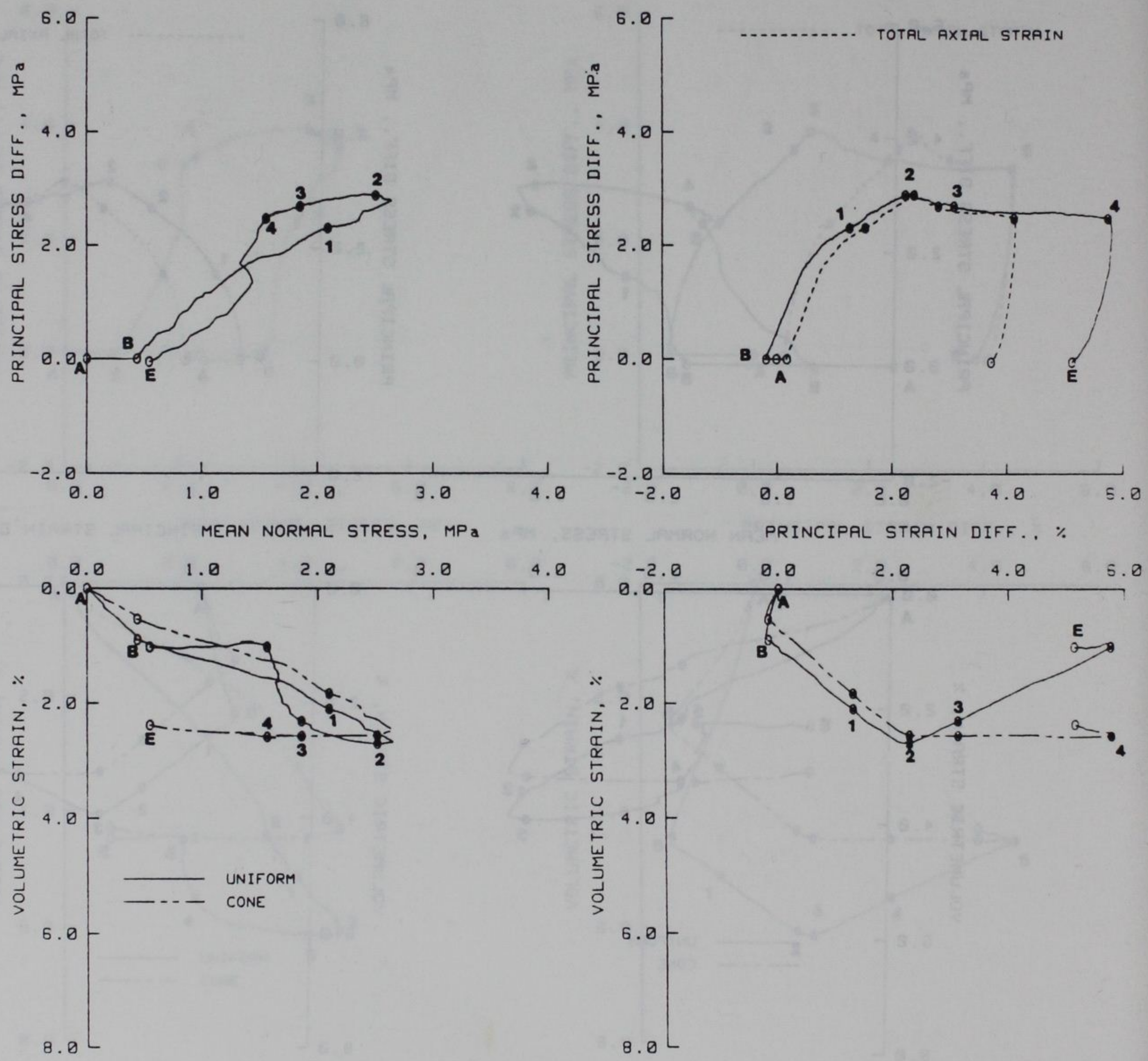


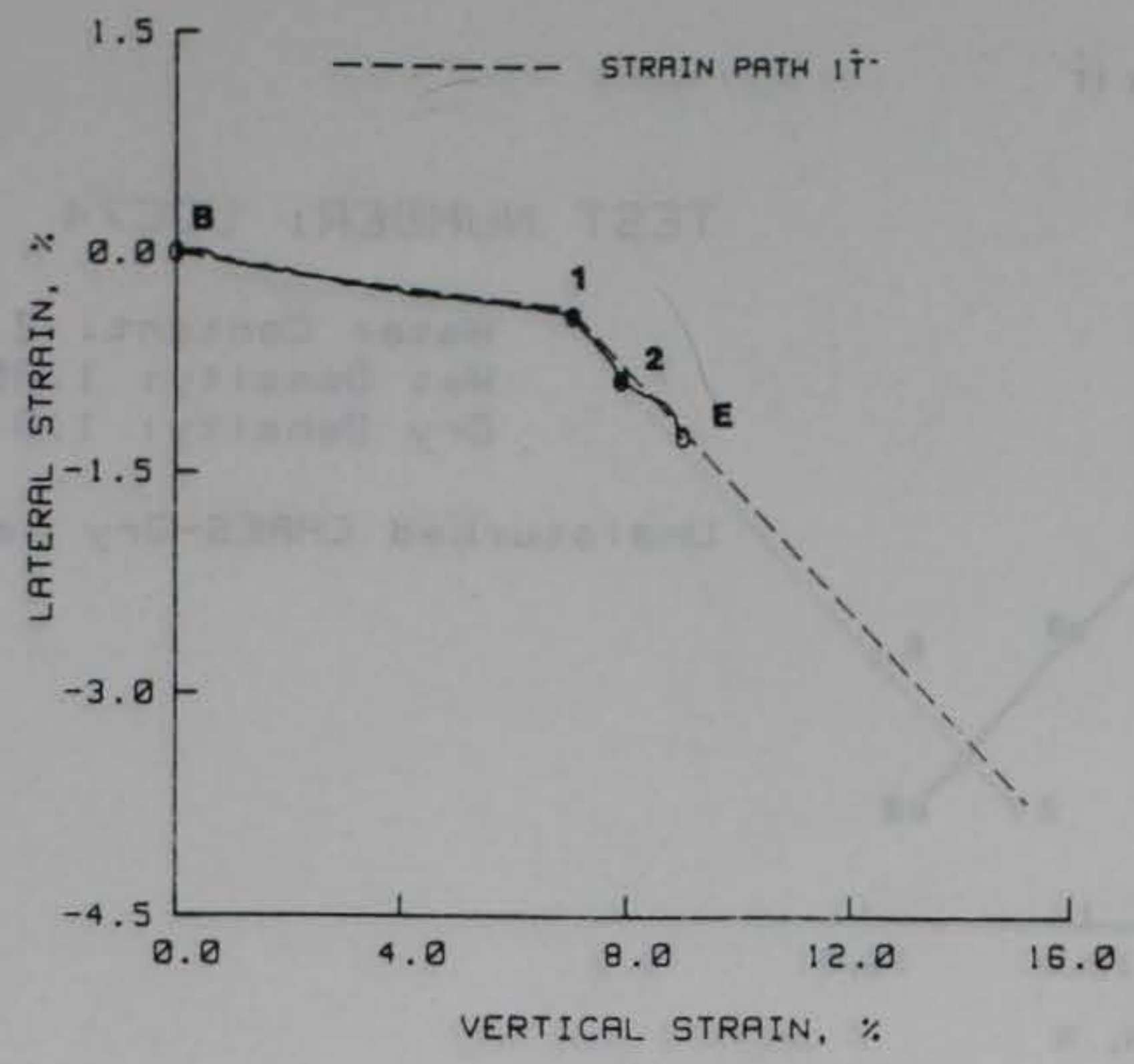

TEST NUMBER: UDC 3

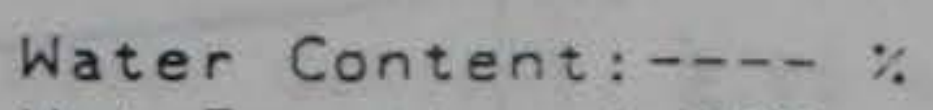

Wet Density: $1.805 \mathrm{~g} / \mathrm{cc}$

Dry Density: $----g^{\prime} \mathrm{co}$

Und isturbed CRRES-Dry Sand

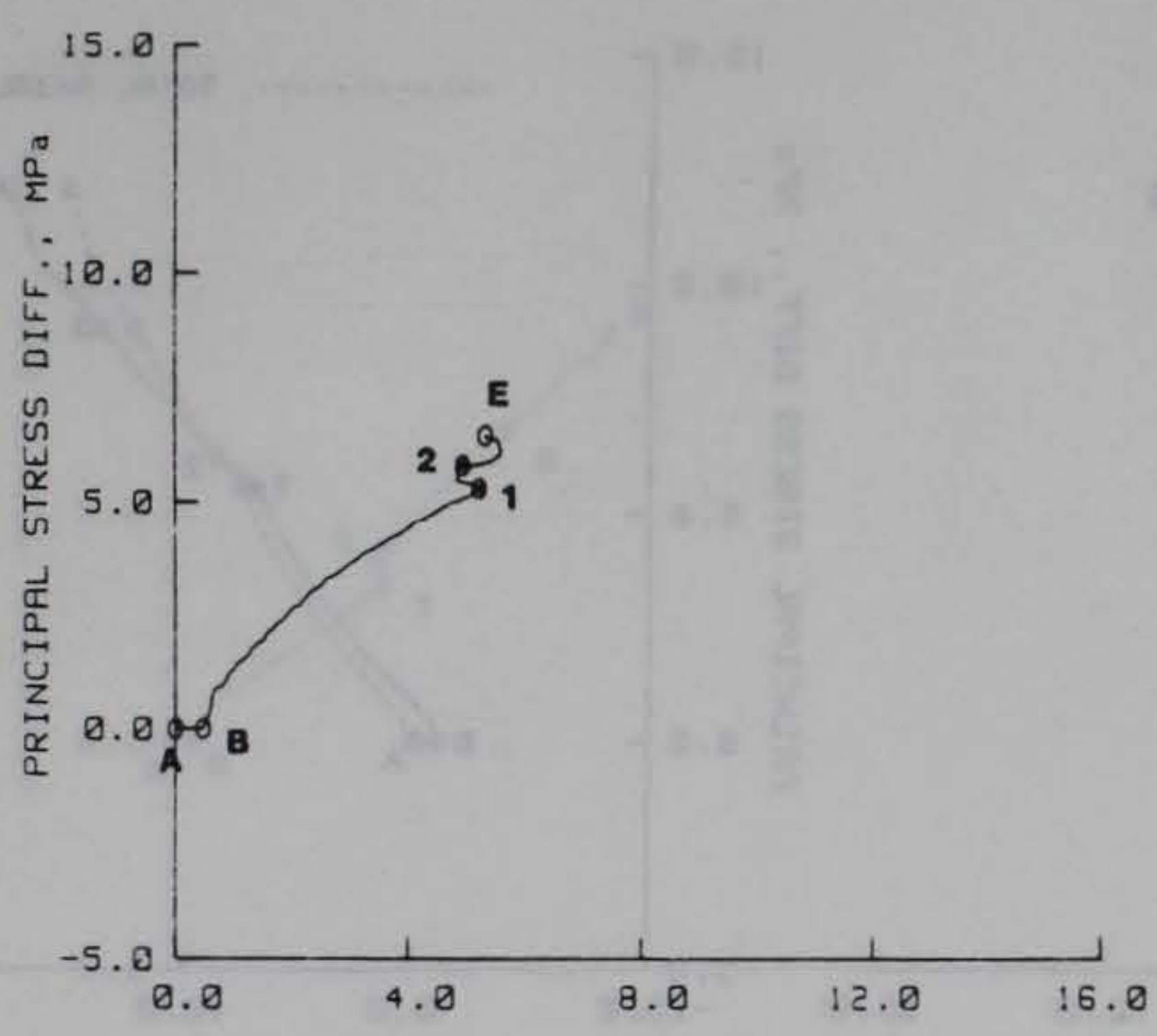

MEAN NORMAL STRESS, MPa

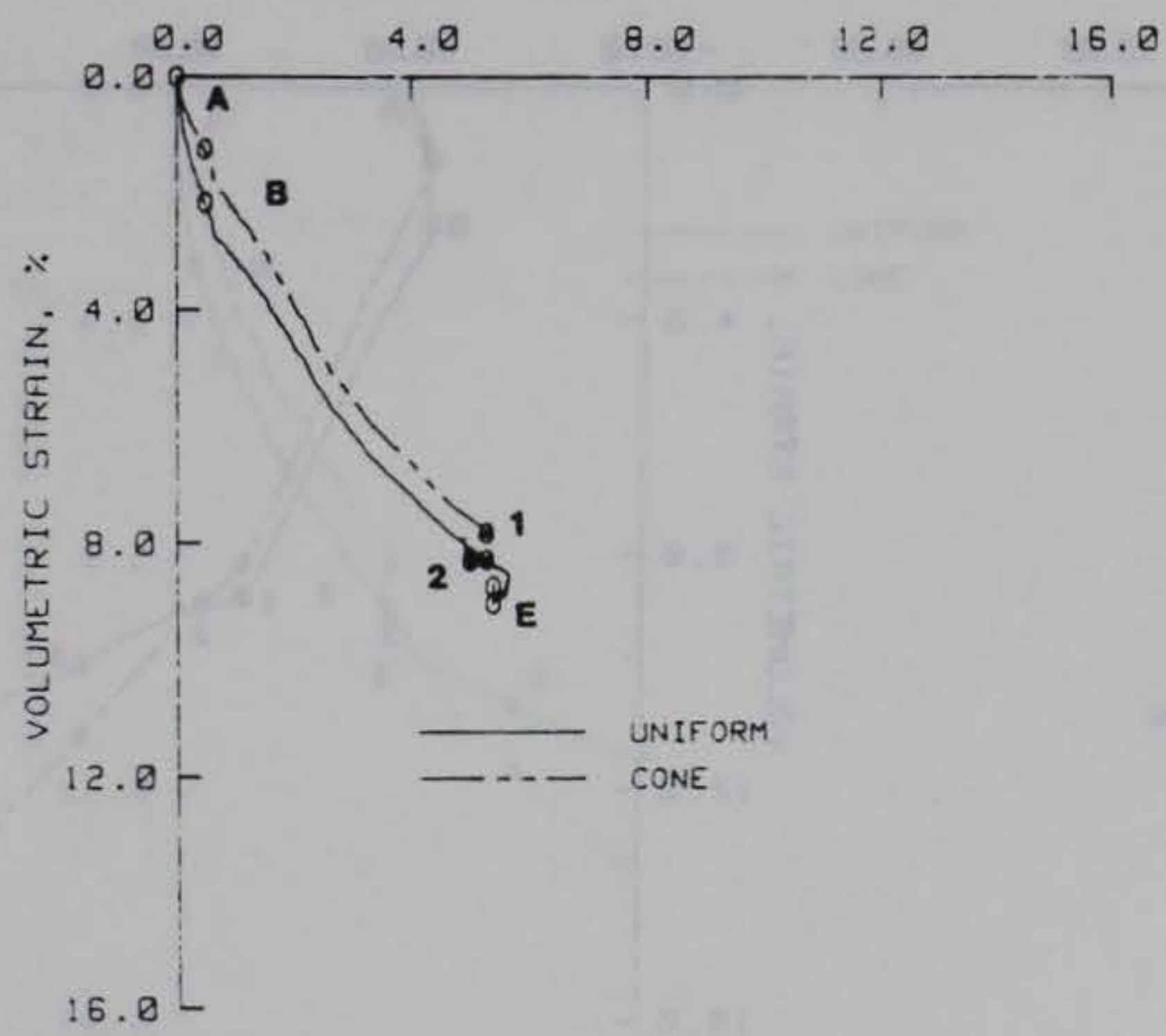

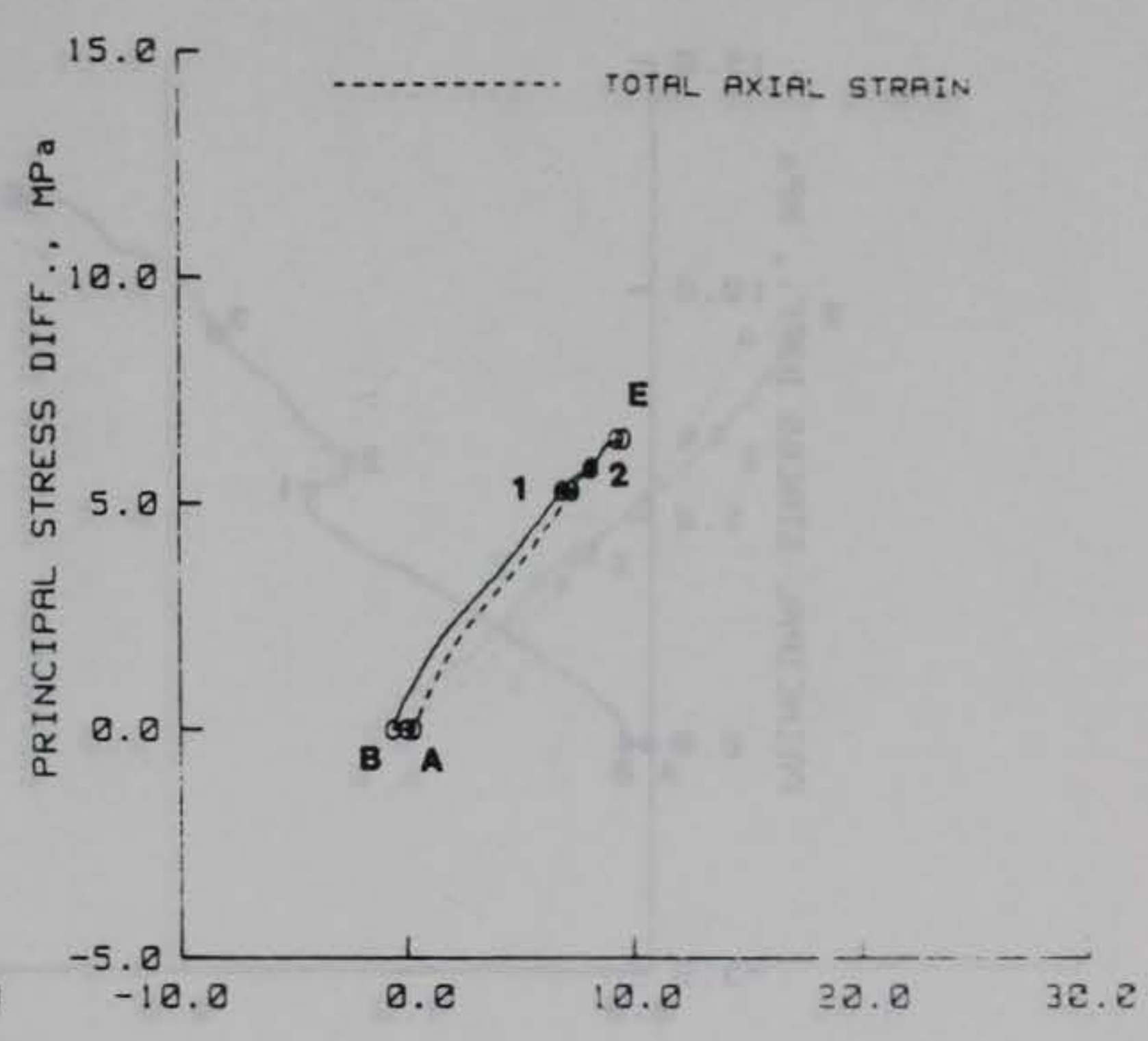
PRINCIPAL STRAIN DIFE., \%

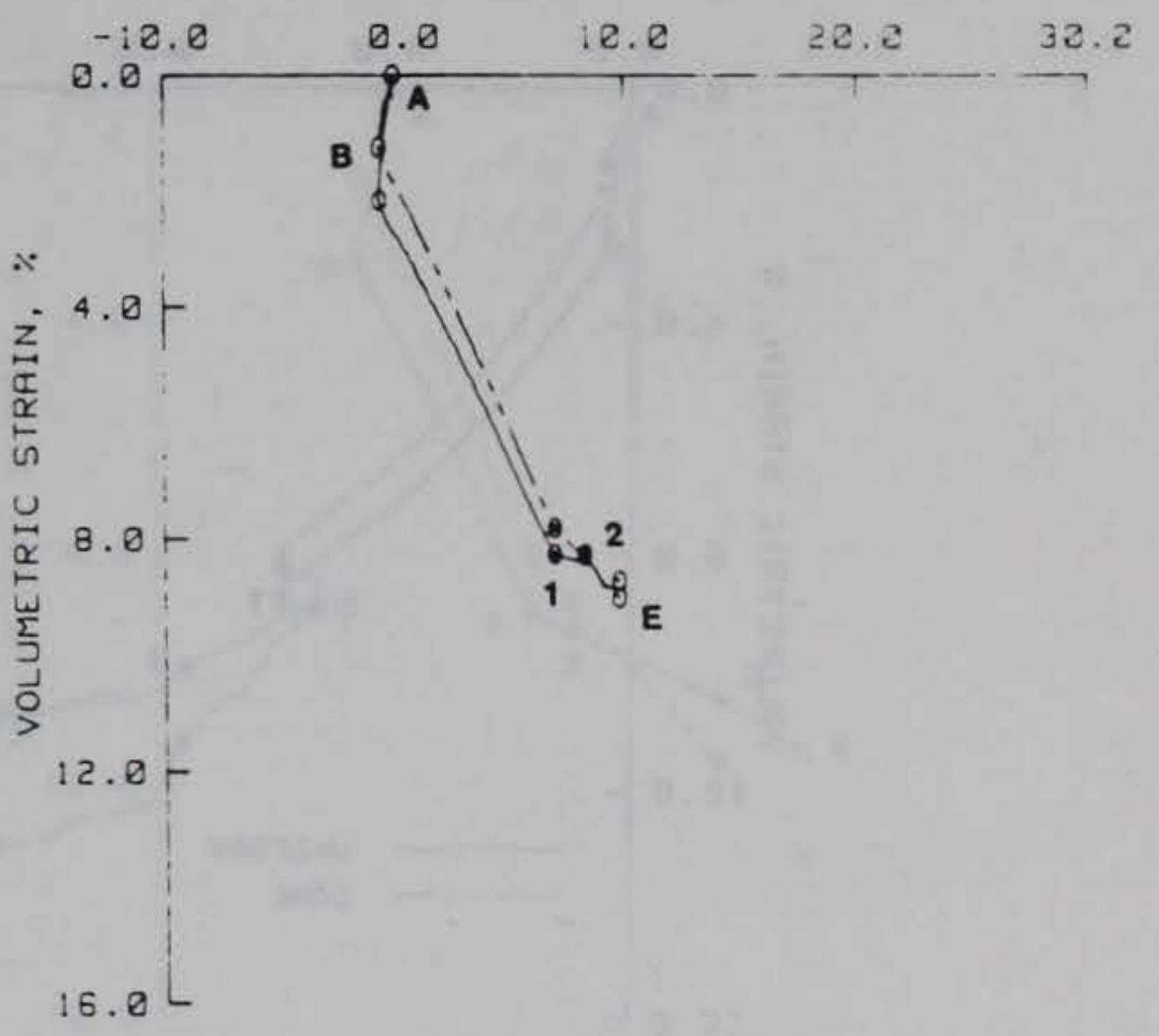




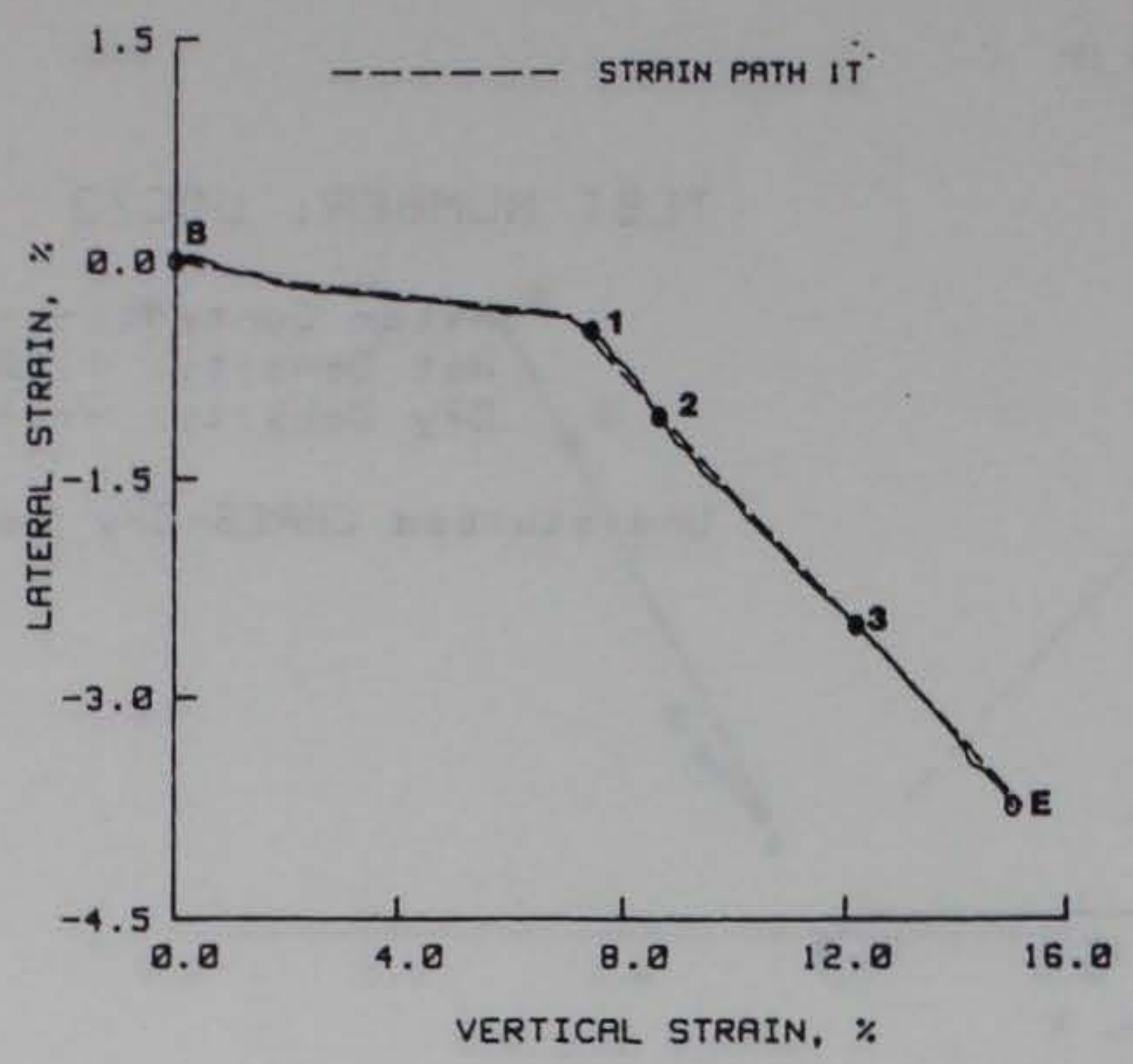

TEST NUMBER: UDC $>4$

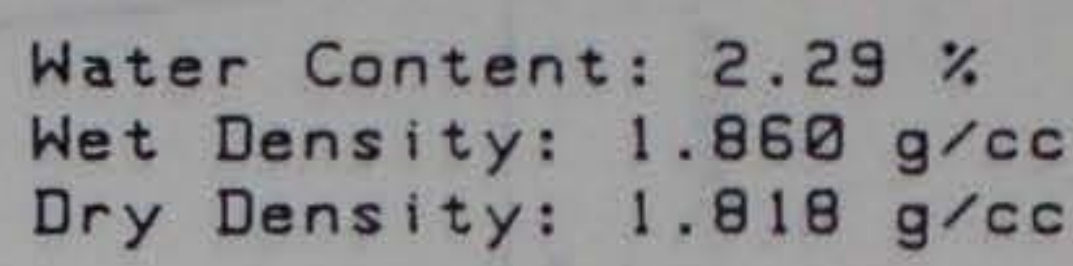

Und isturbed CARES-Dry Sand
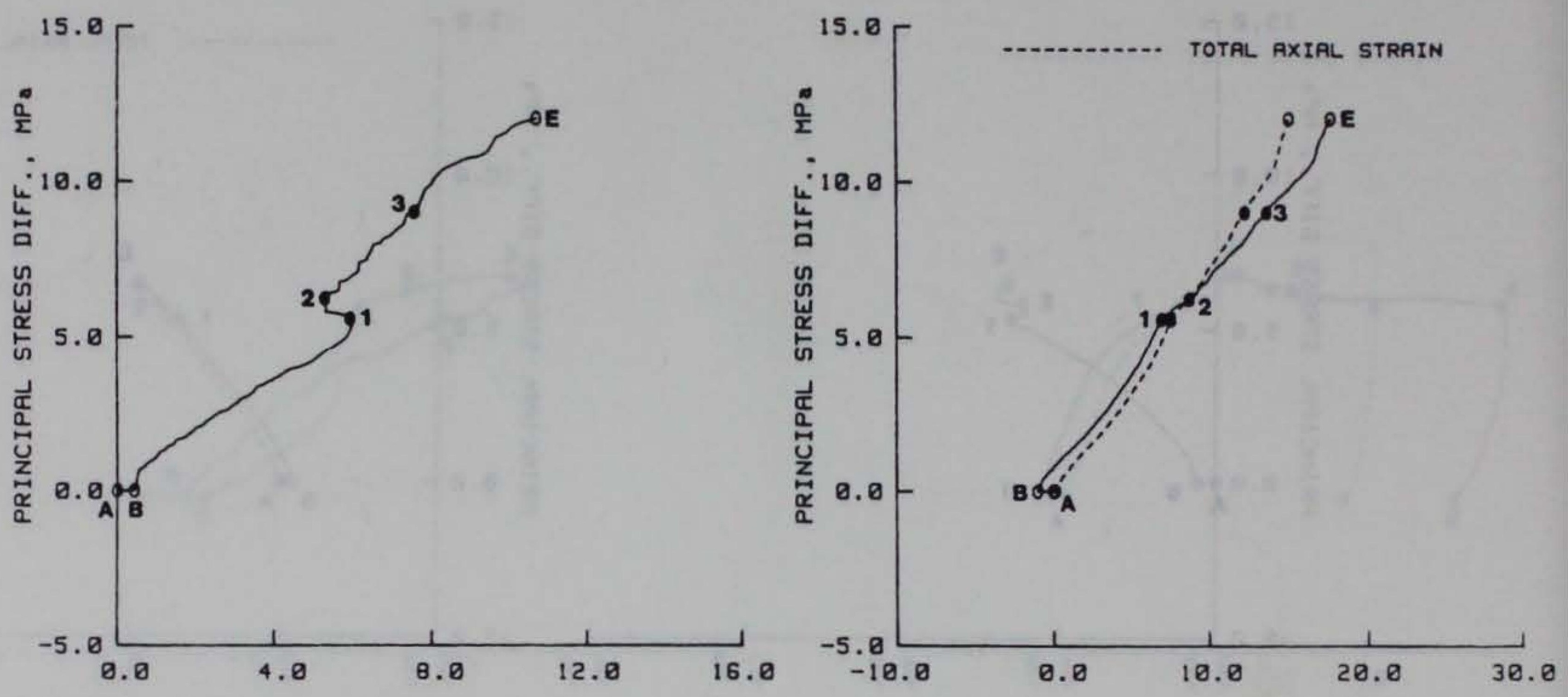
MEAN NORMAL STRESS, MPa
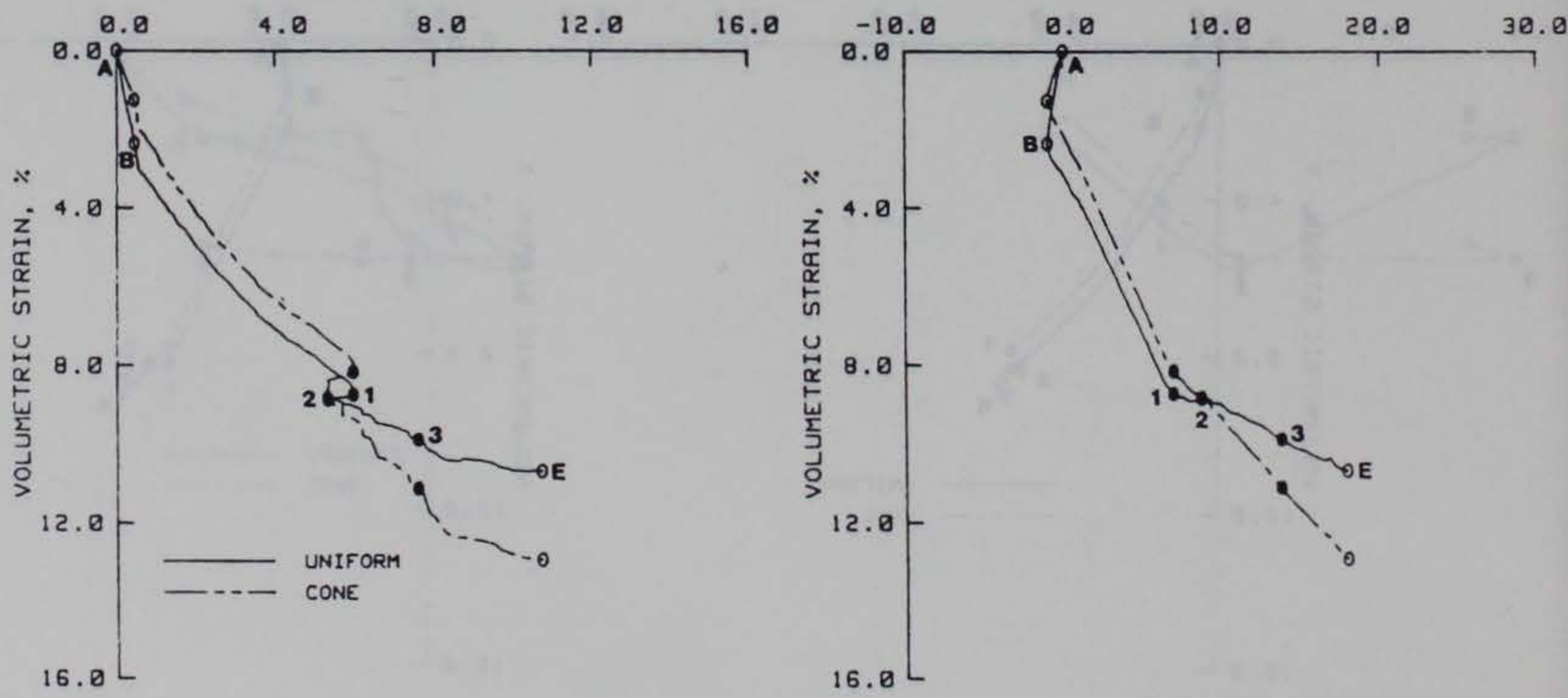

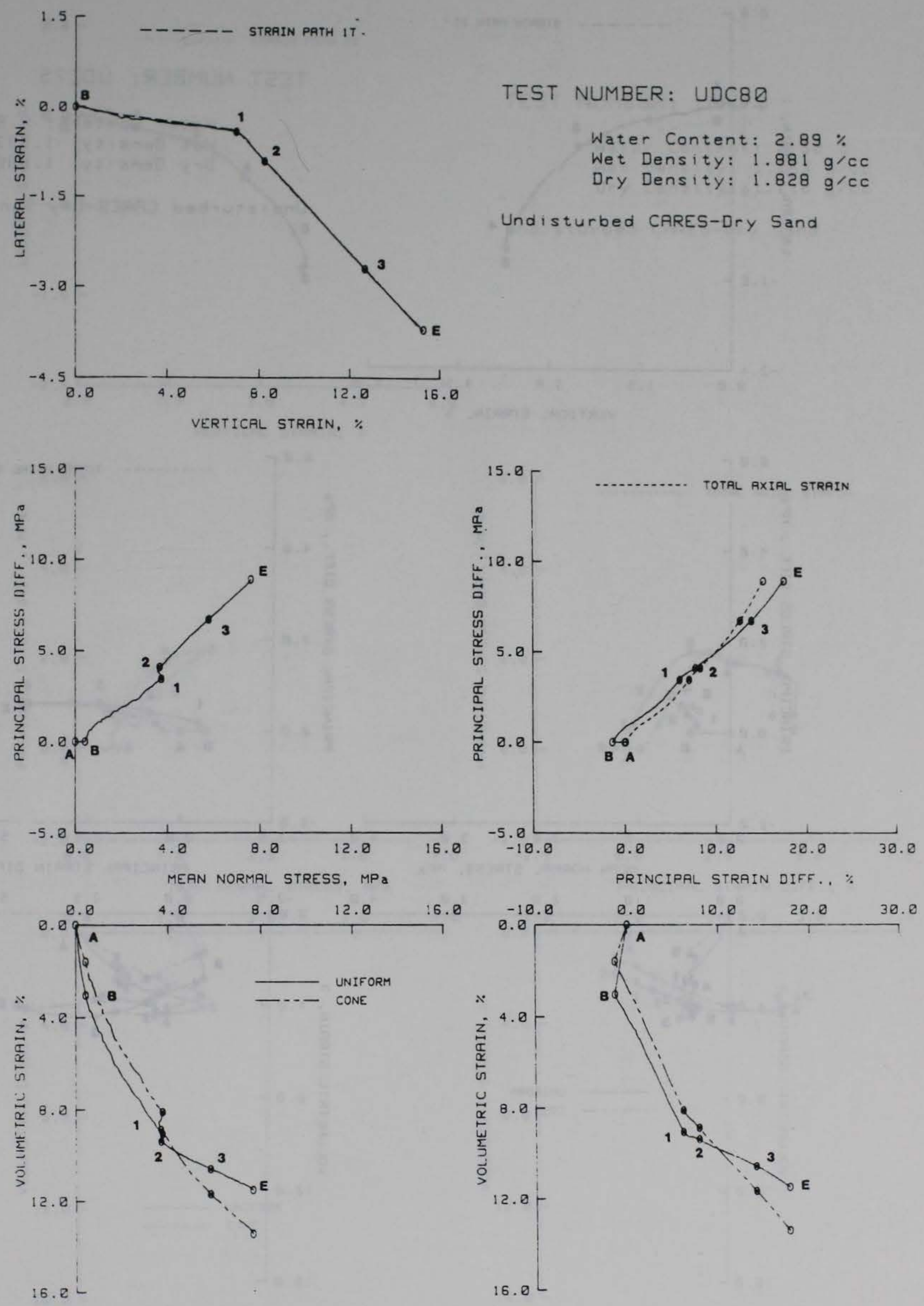


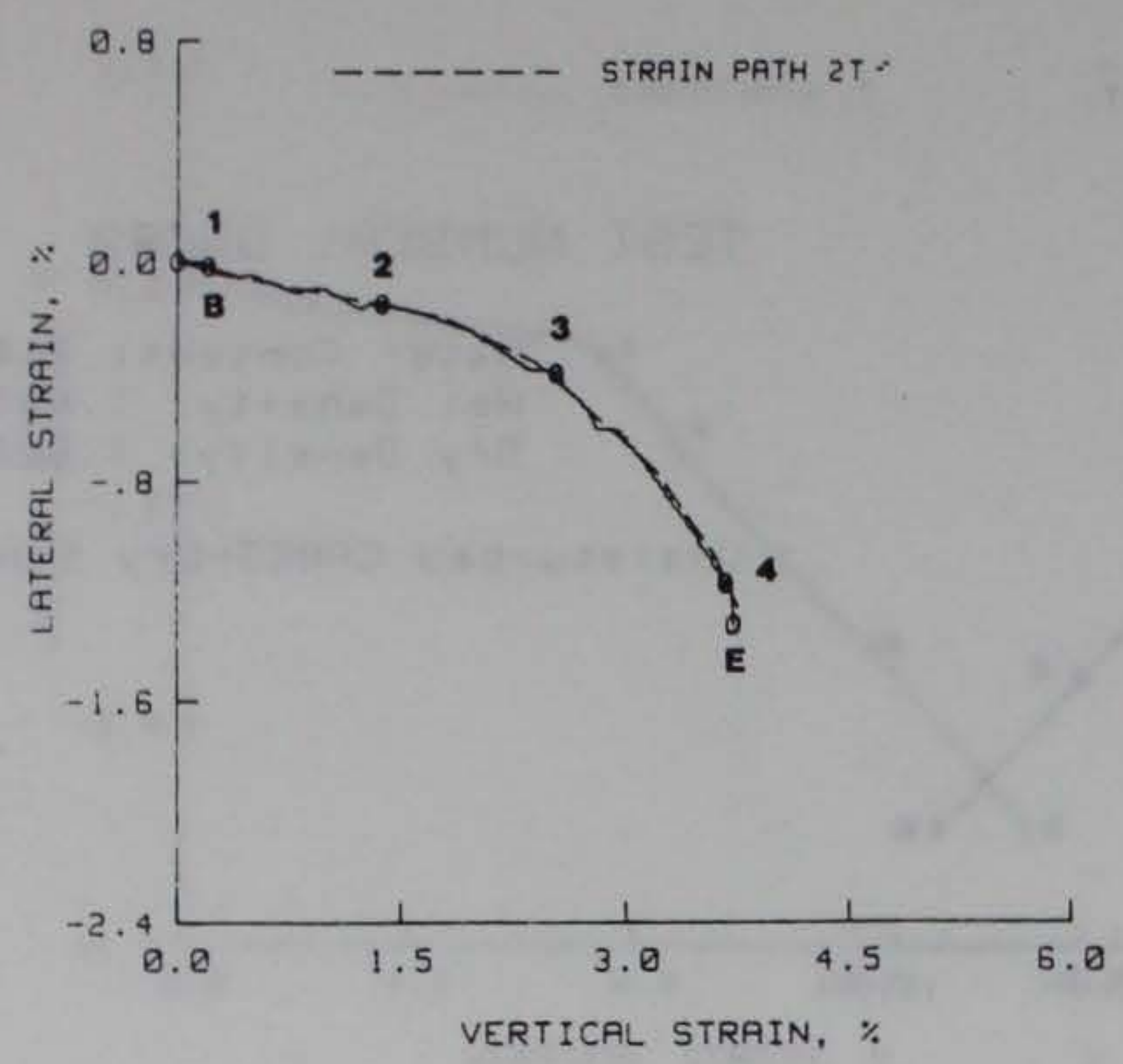

TEST NUMBER: UDC75

Water Content: 8.89 \%

Wet Density: $1.993 \mathrm{~g} / \mathrm{cc}$

Dry Density: $1.830 \mathrm{~g}^{\prime} \mathrm{cc}$

Und isturbed CARES-Dry Sand
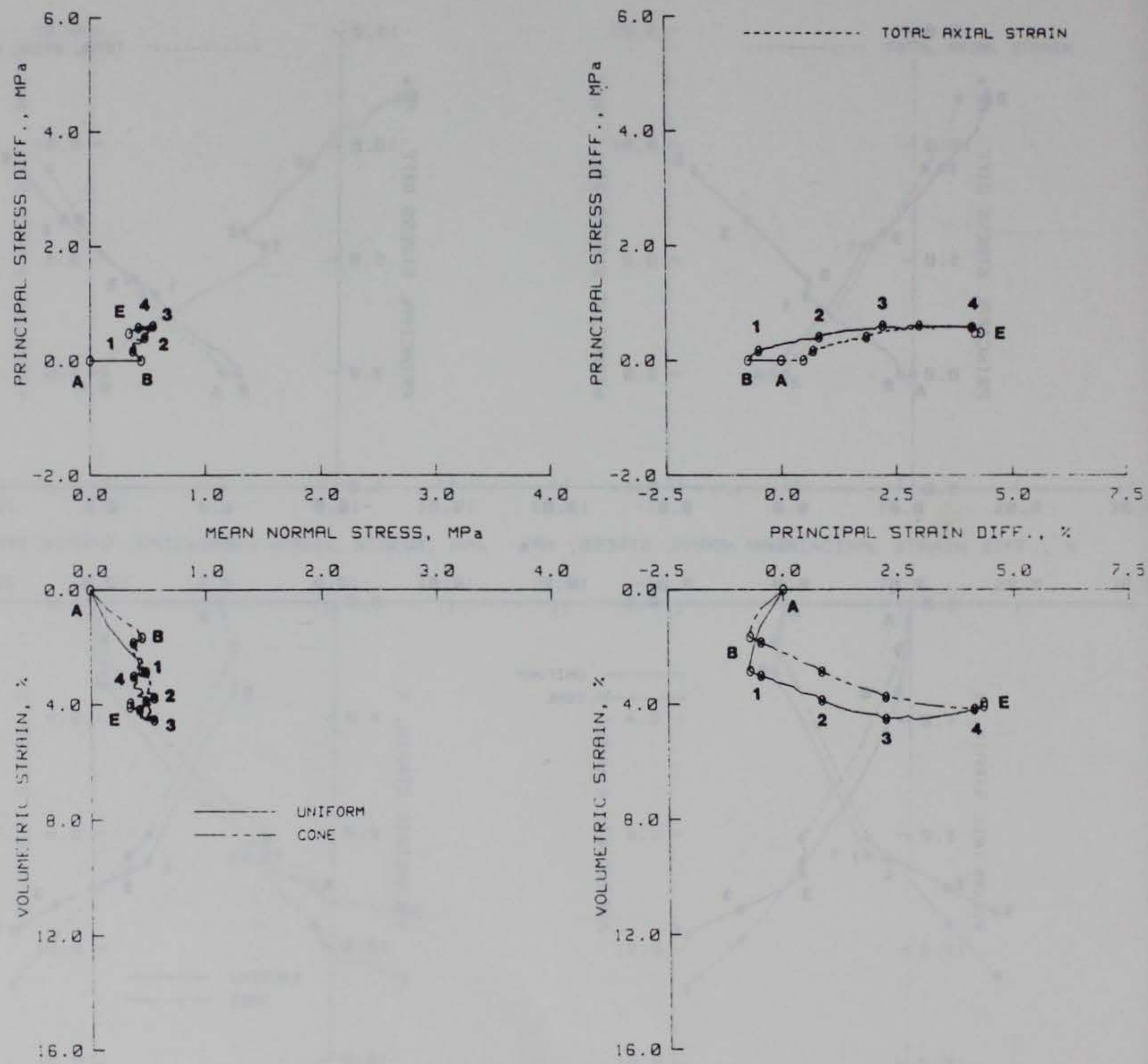


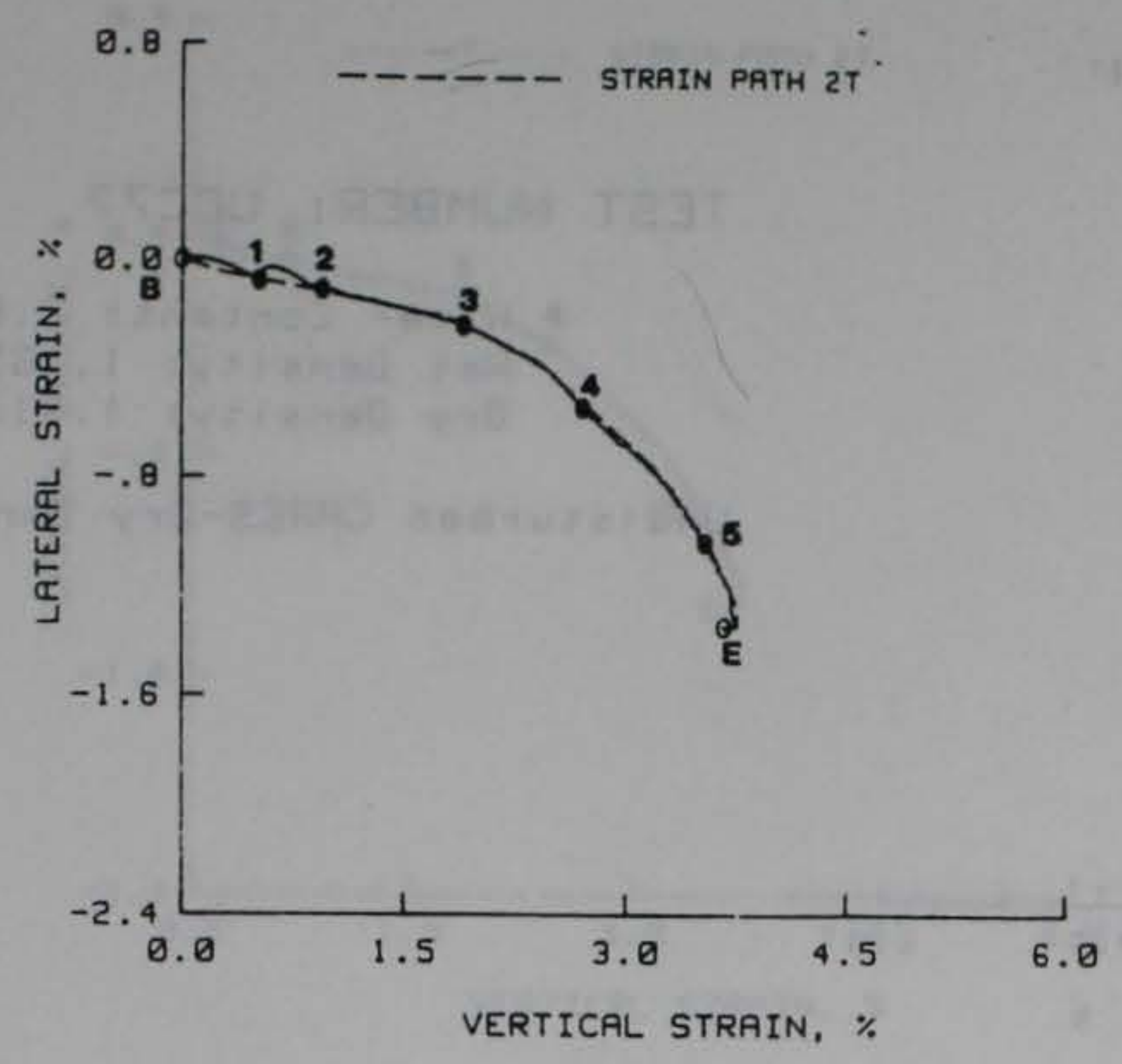

TEST NUMBER: UDCPG

Water Content: $2.62 \%$ Wet Density: $1.763 \mathrm{~g} / \mathrm{cc}$ Dry Density: $1.718 \mathrm{~g} / \mathrm{cc}$

Undisturbed CARES-Dry Sand
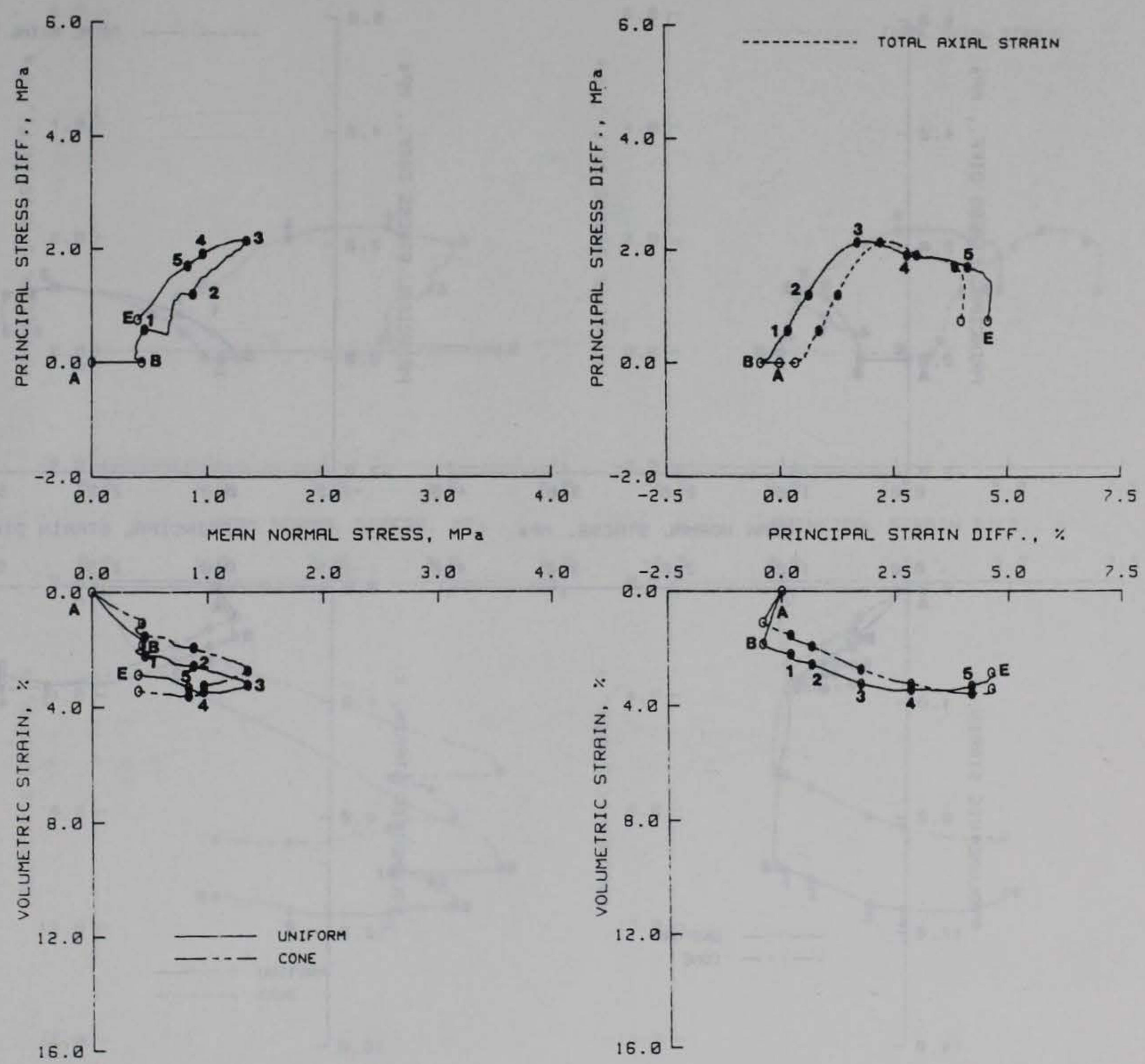


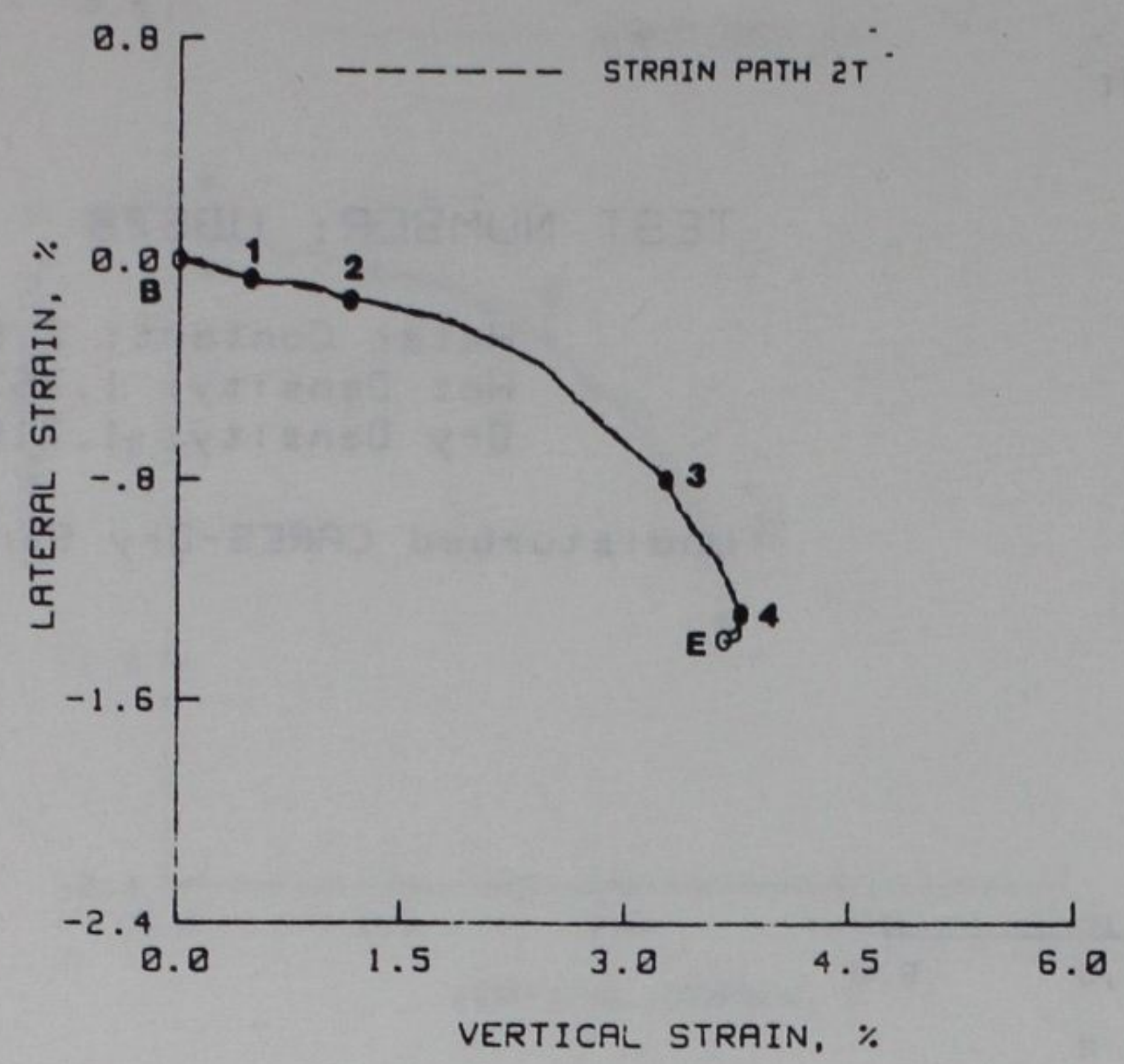

TEST NUMBER: UDCP7

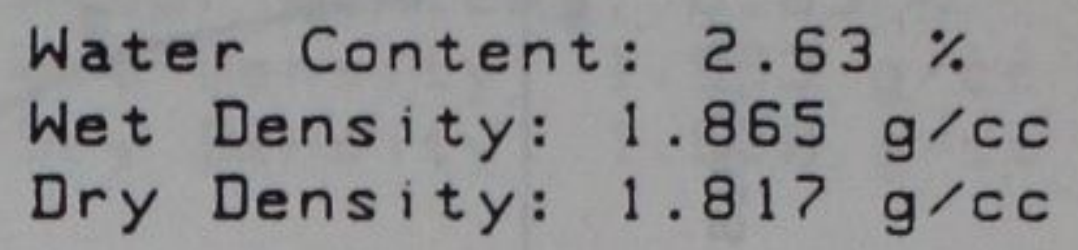

Und isturbed CRRES-Dry Sand
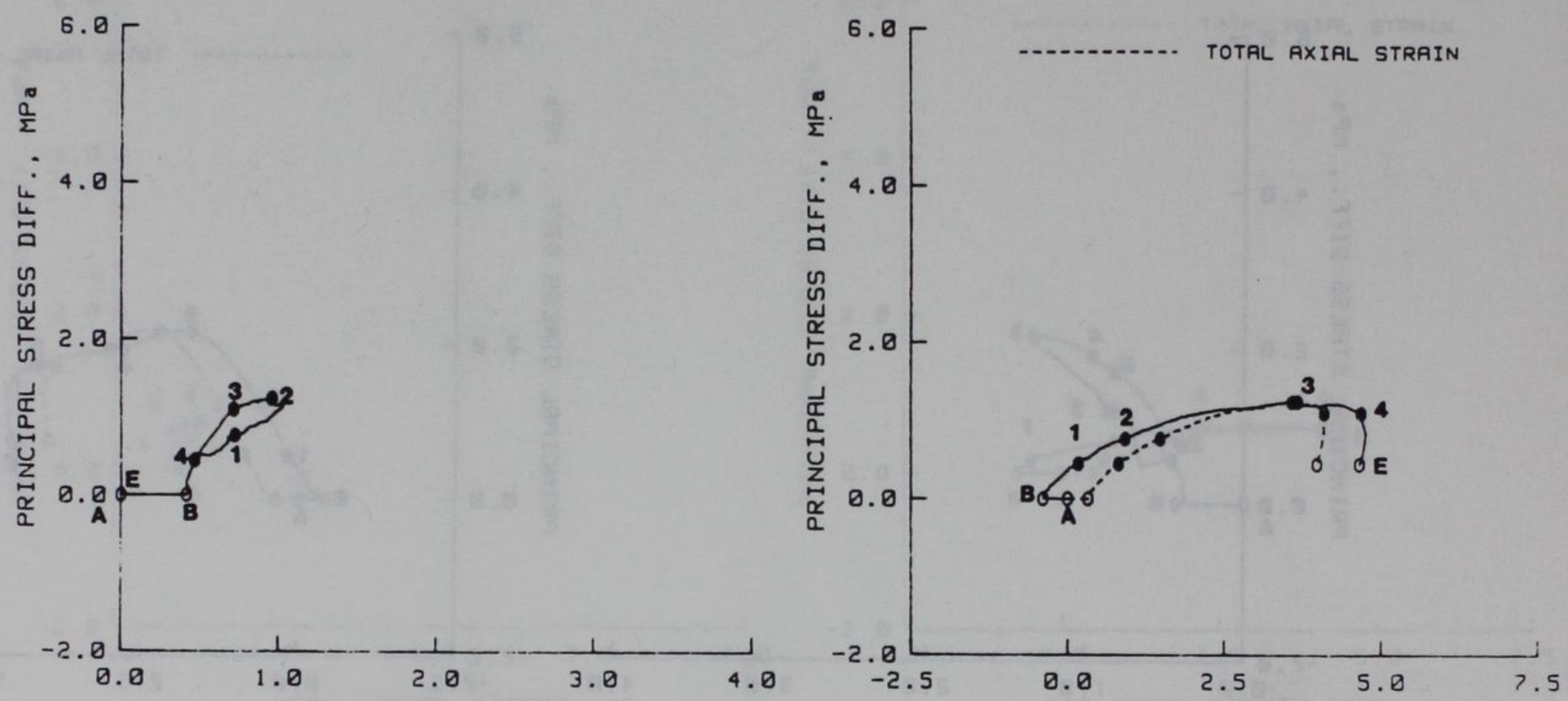

MEAN NORMAL STRESS, MPa

PRINCIPAL STRAIN DIFF., \%
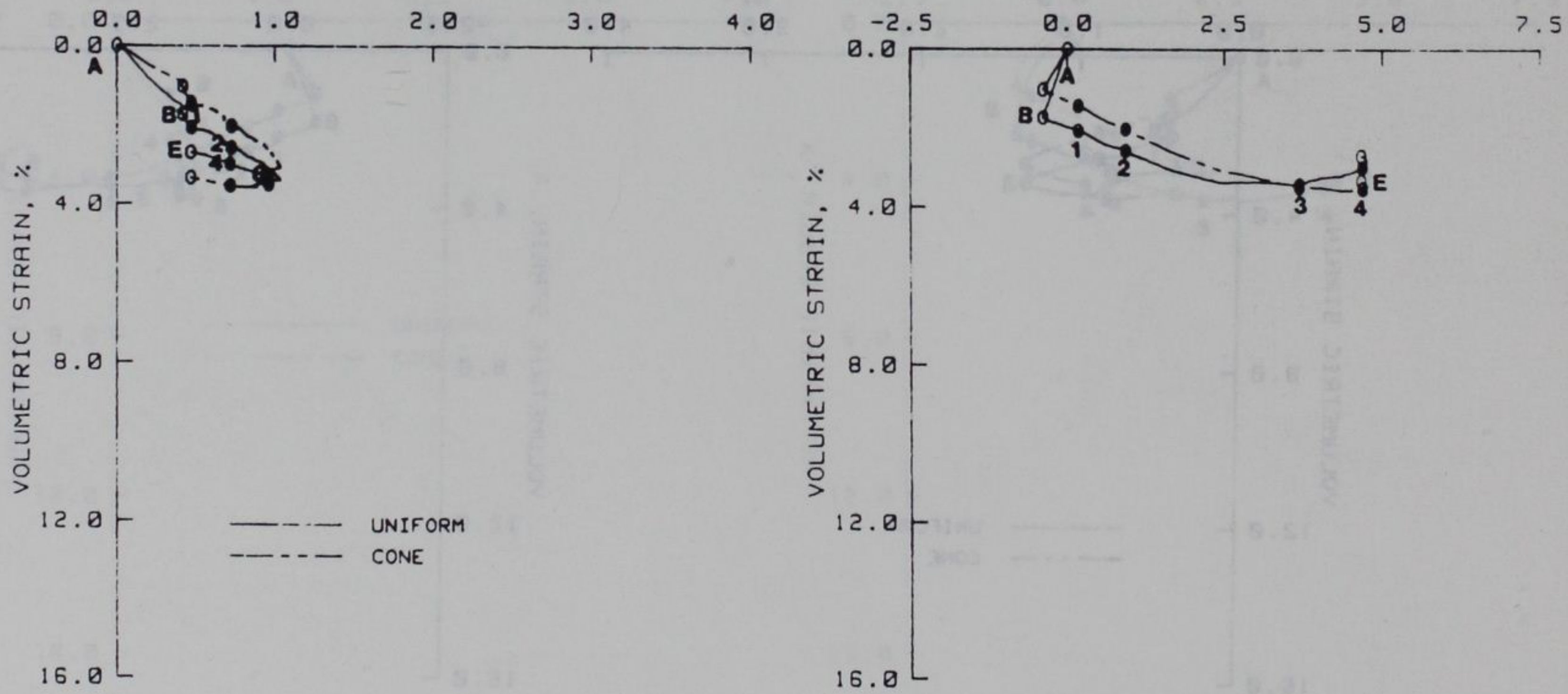


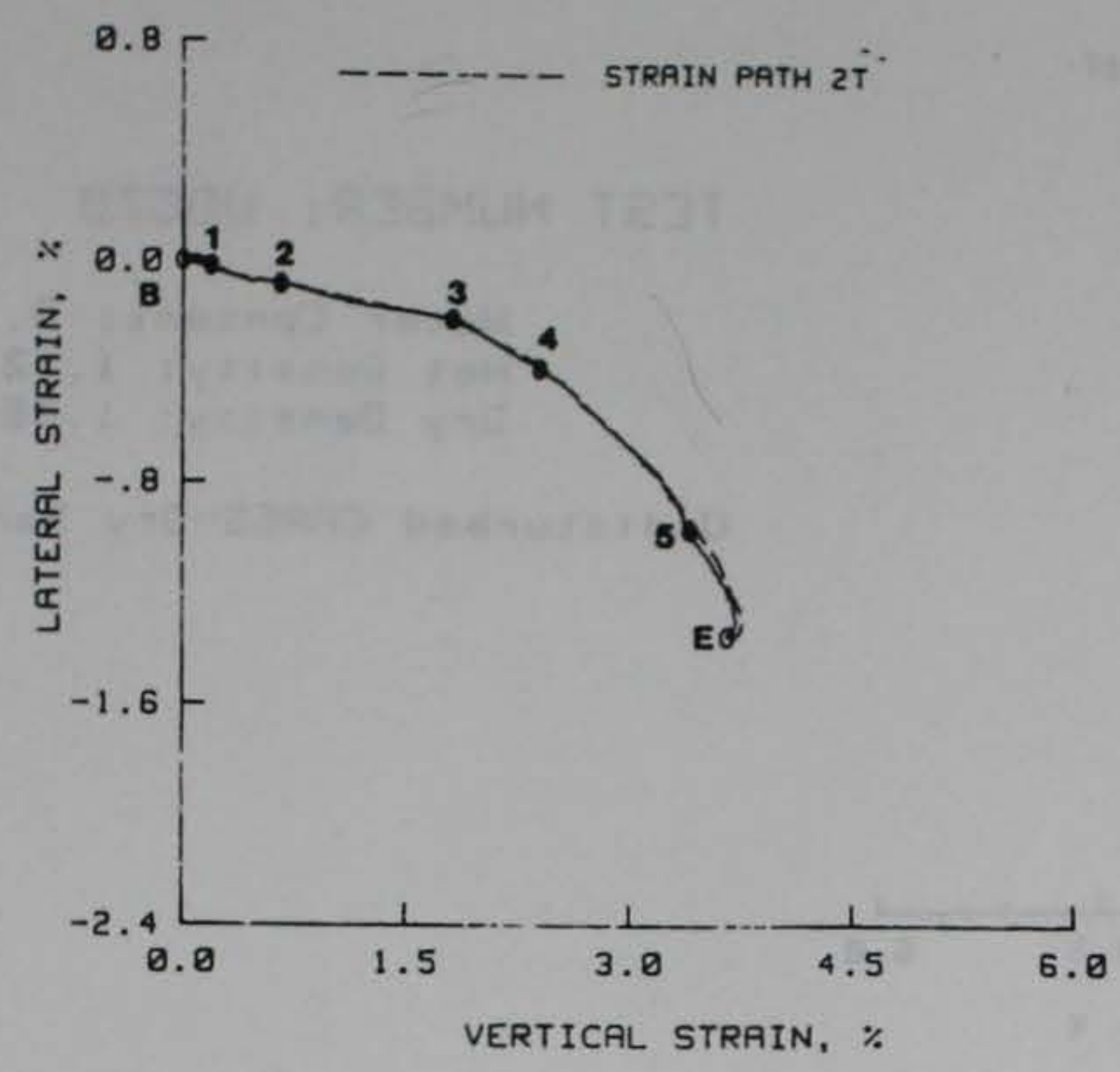

TEST NUMBER: UDC>8

Water Content: $8.50 \%$ Wet Density: $1.878 \mathrm{~g} / \mathrm{cc}$

Dry Density: $1.731 \mathrm{~g} / \mathrm{cc}$

Und isturbed CARES-Dry Sand
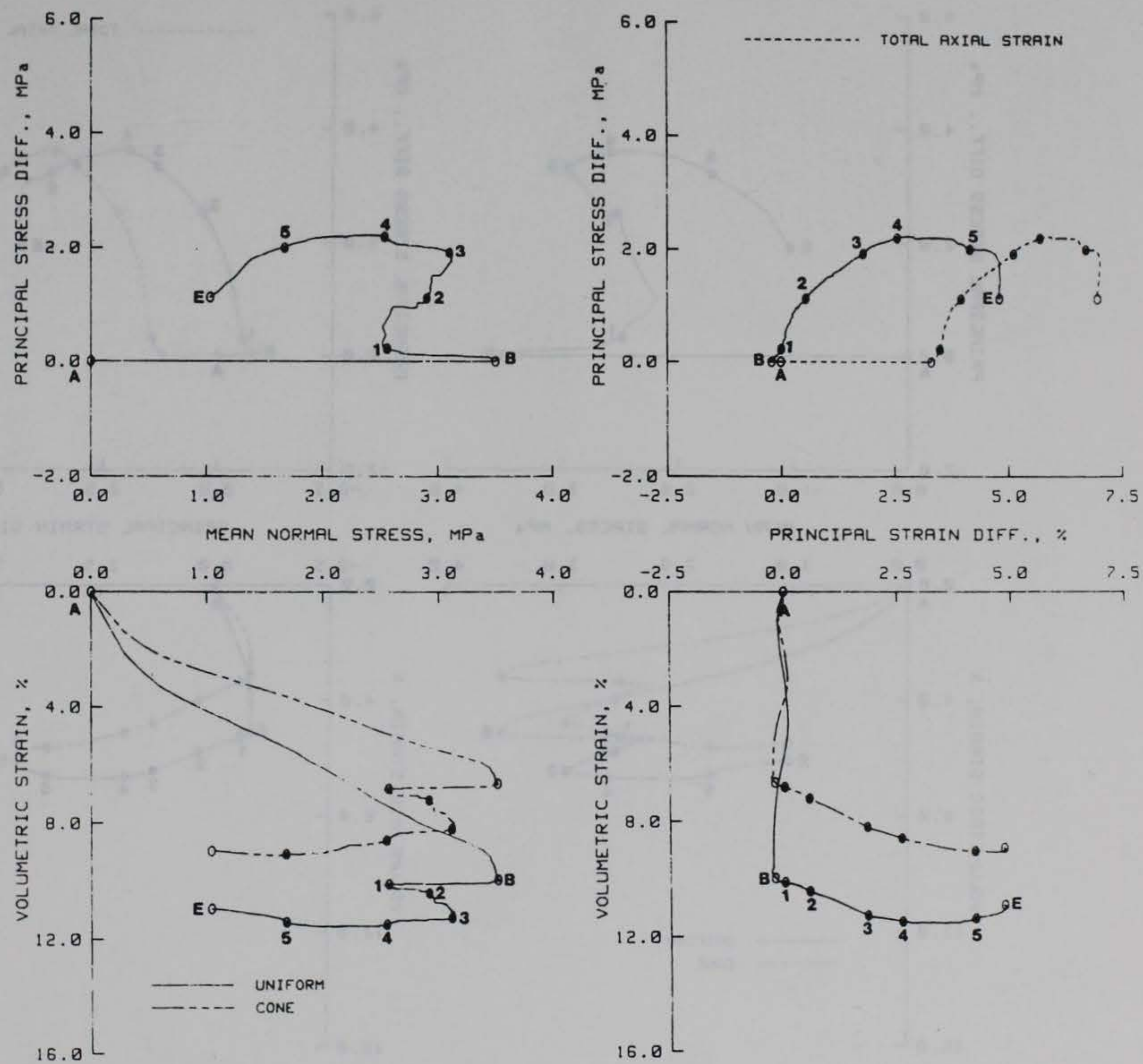

PLATE 74 


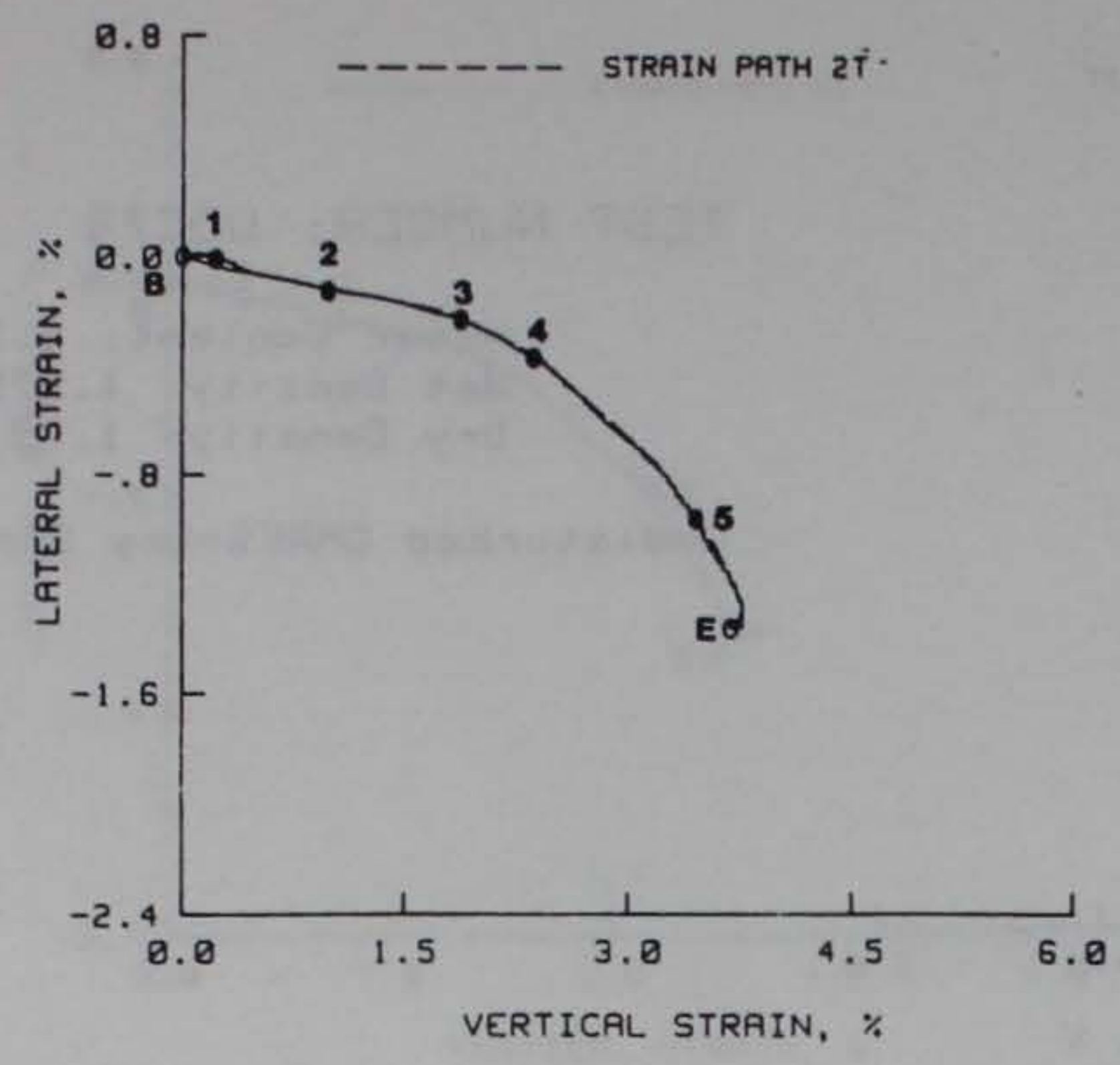

TEST NUMBER: UDC>9

Water Content: $3.77 \%$ Wet Density: $1.729 \mathrm{~g} / \mathrm{cc}$ Dry Density: $1.566 \mathrm{~g} / \mathrm{cc}$

Undisturbed CARES-Dry Sand
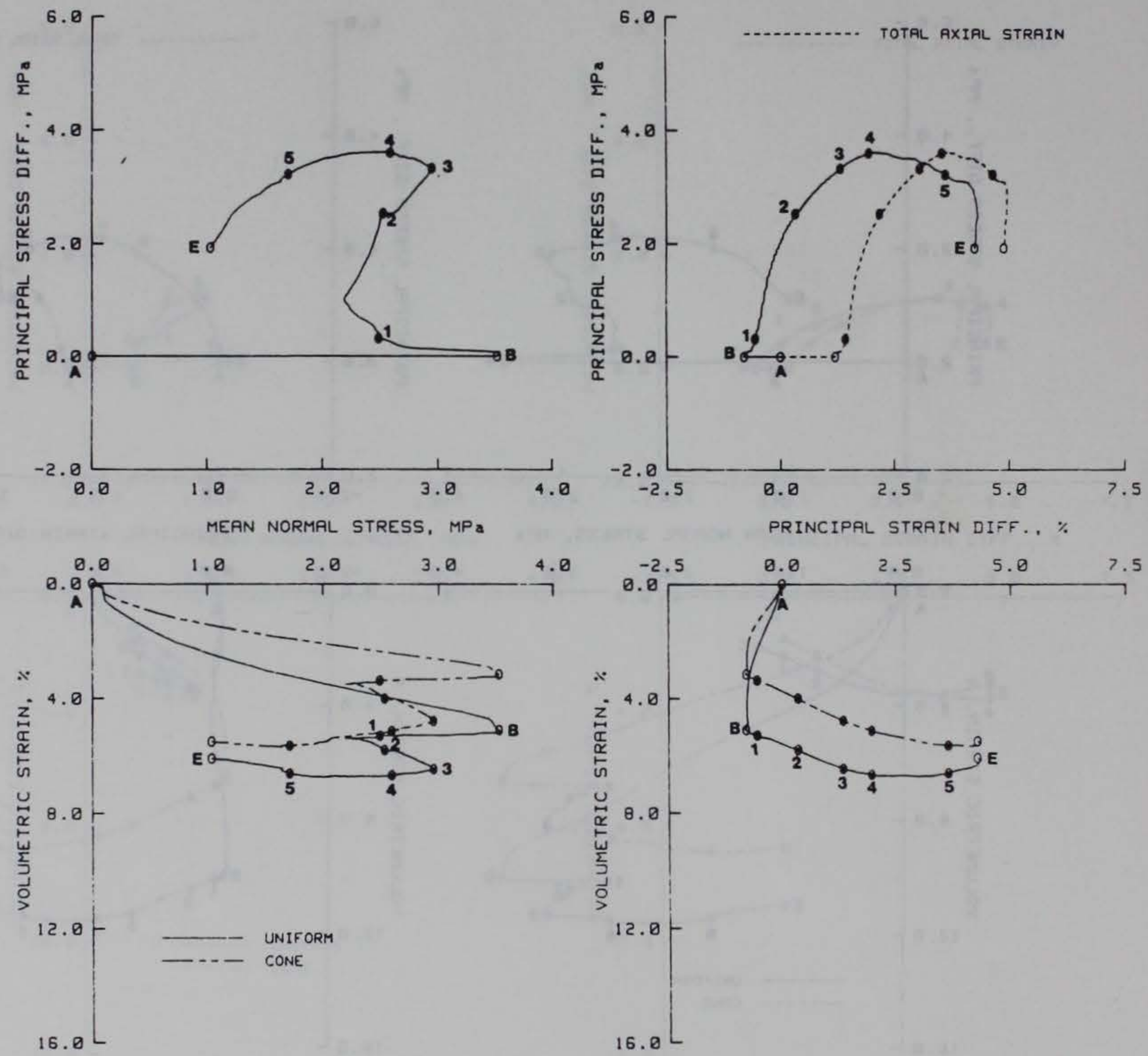